\title{
Introdução às equações diferenciais ordinárias no contexto das funções generalizadas temperadas de Colombeau
}

\author{
Sávio Mendes França
}

DISSERTAÇÃO APRESENTADA

$\mathrm{AO}$

INSTITUTO DE MATEMÁTICA E ESTATÍSTICA

DA

UNIVERSIDADE DE SÃO PAULO

PARA

OBTENÇÃO DO TÍTULO DE MESTRE

EM

CIÊNCIAS

\author{
Área de concentração: Matemática \\ Orientador: Profa. Dra. Roseli Fernandez
}

São Paulo, fevereiro de 2008. 


\title{
Introdução à equações diferenciais ordinárias no contexto das funções generalizadas temperadas de Colombeau
}

\author{
Este exemplar corresponde à redação \\ final da dissertação devidamente corrigida \\ e defendida por Sávio Mendes França
}

e aprovada pela Comissão Julgadora.

São Paulo, 21 de fevereiro de 2008.

Banca Examinadora:

- Profa ${ }^{a}$ Dra. Roseli Fernandez (orientadora) - IME-USP

- Prof. Dr. Jorge Aragona - IME-USP

- Prof. Dr. Francisco Villarreal Alvarado - FEIS-UNESP 
à toda minha família, de modo especial aos meus pais, à minha esposa Delma e aos meus filhos Marina e Renato 



\section{Resumo}

O objetivo deste trabalho é estudar, sob que condições, o problema de valor inicial associado a uma equação diferencial ordinária de primeira ordem, no contexto das funções generalizadas temperadas de Colombeau, admite pelo menos uma (ou somente uma) solução generalizada ou solução generalizada temperada. Para essa finalidade estudamos algumas propriedades das funções generalizadas, das funções generalizadas temperadas e das funções generalizadas temperadas na segunda variável. Além do estudo dessas propriedades, apresentamos uma imersão do espaço das distribuições na álgebra das funções generalizadas de Colombeau e uma imersão do espaço das distribuições temperadas na álgebra das funções generalizadas temperadas de Colombeau. Finalizamos o trabalho estudando, no contexto das funções generalizadas temperadas de Colombeau, uma equação de Euler-Lagrange e solução para frente em sistemas autônomos.

Palavras-chave: funções generalizadas, equação diferencial ordinária, distribuições. 



\section{Abstract}

The objective of this work is to study, under which conditions, the initial value problem associated with a first-order ordinary differential equation, in the framework of Colombeau's tempered generalized functions, it admits at least one (or only one) generalized solution or generalized tempered solution. For this purpose we studied some properties of the generalized functions, of the generalized tempered functions and the generalized tempered functions in the second variable. Besides the study of these properties, we present an embedding of the space of distributions into the algebra of Colombeau's generalized functions and an embedding of the space of tempered distributions into the algebra of Colombeau's tempered generalized functions. We end the work studying, in the framework of Colombeau's tempered generalized functions, an Euler-Lagrange equation and forward solution for autonomous system.

Keywords: generalized functions, ordinary differential equation, distributions. 



\section{Agradecimentos}

Quero agradecer, em primeiro lugar, à Prof ${ }^{a}$ Roseli Fernandez, que me orientou durante todo o período de elaboração deste trabalho, com quem tive a honra de conviver e muito aprendi e, pela qual, tenho extrema admiração e respeito.

Agradeço a todo os professores do IME que, de forma direta ou indireta, contribuiram para a minha formação. Em especial, agradeço às professoras Elza Furtado Gomide e Maria Lúcia Sobral Singer, que me orientaram no período no qual fiz a especialização e que, somente agora, pude tornar pública a minha admiração.

Agradeço aos amigos com os quais convivi neste instituto e cuja amizade, por si só, foi um grande incentivo.

Por fim, Agradeço à Deus, pela graça da vida, pela minha família e pela oportunidade de realizar mais um sonho. 



\section{Sumário}

Introdução

1 Algumas álgebras de Colombeau e suas propriedades $\quad 1$

1.1 A álgebra $\overline{\mathbb{R}} \ldots \ldots \ldots \ldots \ldots$

1.2 A álgebra $\mathcal{G}\left(\Omega ; \mathbb{R}^{m}\right) \ldots \ldots \ldots \ldots$

1.3 A álgebra $\mathcal{G}_{\tau}\left(\Omega ; \mathbb{R}^{m}\right) \ldots \ldots \ldots \ldots$. . . . . . . . . . . . . . . . . . . .

1.4 A álgebra $\widetilde{\mathcal{G}}_{\tau}\left(\Omega_{1} \times \Omega_{2} ; \mathbb{R}^{m}\right) \ldots \ldots \ldots$

1.5 As álgebras $\mathcal{G}\left(\left[t_{0},+\infty\left[; \mathbb{R}^{m}\right)\right.\right.$ e $\mathcal{G}_{\tau}\left(\left[t_{0},+\infty\left[; \mathbb{R}^{m}\right) \ldots \ldots . \ldots . . . . . .42\right.\right.$

2 Distribuições e funções generalizadas de Colombeau 51

2.1 Os conjuntos $\mathcal{D}^{\prime}(\Omega ; \mathbb{R})$ e $\mathcal{S}^{\prime}\left(\mathbb{R}^{n} ; \mathbb{R}\right) \ldots \ldots \ldots$. . . . . . . . . . . 51

2.2 Uma imersão de $\mathcal{D}^{\prime}(\Omega ; \mathbb{R})$ em $\mathcal{G}(\Omega ; \mathbb{R}) \ldots \ldots$. . . . . . . . . . . . . . . . . 68

2.3 Uma imersão de $\mathcal{S}^{\prime}\left(\mathbb{R}^{n} ; \mathbb{R}\right)$ em $\mathcal{G}_{\tau}\left(\mathbb{R}^{n} ; \mathbb{R}\right) \ldots \ldots$. . . . . . . . . . . . . 91

3 Equações diferenciais ordinárias $\quad 101$

3.1 Existência e unicidade de soluções . . . . . . . . . . . . . . . . . . . . 102

3.2 As equações de Euler-Lagrange - um exemplo . . . . . . . . . . . . . . . . 138

3.3 Solução para frente em sistemas autônomos . . . . . . . . . . . . . . 150

$\begin{array}{ll}\text { Lista de símbolos e notações } & 169\end{array}$

$\begin{array}{ll}\text { Referências bibliográficas } & 175\end{array}$ 


\section{Introdução}

J. F. Colombeau, na década de 80, com a finalidade de resolver o Problema da Multiplicação de Distribuições, introduziu a Teoria das Funções Generalizadas. As primeiras aplicações foram realizadas na área das equações diferenciais. Recentemente, há trabalhos nas áreas de geometria, álgebra e análise funcional.

Dentre as álgebras que existem na Teoria das Funções Generalizadas, iremos trabalhar com a álgebra dos números reais generalizados, com a das funções generalizadas, com a das funções generalizadas temperadas e com algumas variações dessas álgebras. O propósito deste trabalho é apresentar, no contexto das álgebras das funções generalizadas temperadas, o problema de valor inicial associado a uma equação diferencial ordinária de primeira ordem. Esta Dissertação foi baseada no estudo do capítulo 1 de [GKOS] e na leitura de [Del].

Iniciamos o nosso trabalho apresentando no capítulo 1 as principais propriedades das álgebras de Colombeau que serão utilizadas. Na seção 1.1 definimos a álgebra dos números reais generalizados. A seguir estudamos a álgebra das aplicações generalizadas. Destacamos o teorema de nulidade para funções moderadas (Teorema 1.2.5). Esse teorema, extraído de [GKOS], é muito útil para provar que uma determinada função é nula. Na seção 1.3 apresentamos algumas propriedades da álgebra das aplicações generalizadas temperadas. Em [GKOS] encontra-se um teorema de nulidade para funções moderadas temperadas definidas em abertos "box". Ao analisar a sua prova, percebemos que esse teorema pode ser aplicado a uma classe maior de abertos. Essa extensão encontra-se no Teorema 1.3.7. Na próxima seção estudamos a álgebra das aplicações que apresentam na segunda variável uma característica similar à da algebra das aplicações generalizadas temperadas. Por esse motivo 
optamos por chamá-la de álgebra das aplicações generalizadas temperadas na segunda variável. Finalizamos o capítulo apresentando a álgebra das aplicações generalizadas em $\left[t_{0},+\infty\left[\right.\right.$ a valores em $\mathbb{R}^{m}$ e a álgebra das aplicações generalizadas temperadas em $\left[t_{0},+\infty[\right.$ a valores em $\mathbb{R}^{m}$, onde $t_{0} \in \mathbb{R}$. Nestas duas últimas seções destacamos o Teorema 1.5.5 e o Teorema 1.5.6 que foram baseados no estudo das provas dos teoremas de nulidade das seções 1.2 e 1.3 .

Tendo em vista que a origem da Teoria das Funções Generalizadas de Colombeau foi motivada pelas distribuições, nos pareceu interessante mostrar, no capítulo 2, como as distribuições podem ser interpretadas como funções generalizadas. Para facilitar a leitura para um leitor pouco familiarizado com a Teoria das Distribuições, apresentamos na seção 2.1 um breve estudo das distribuições e das distribuições temperadas. Nas próximas duas seções definimos uma imersão do espaço das distribuições na álgebra das funções generalizadas e uma imersão do espaço das distribuições temperadas na álgebra das funções generalizadas temperadas. Essas imersões foram baseadas nos trabalhos [Dec] e [GKOS].

O capítulo 3 é dedicado ao principal objeto de estudo desta Dissertação, ou seja, às equações diferenciais ordinárias no contexto das funções generalizadas temperadas. Na seção 3.1 apresentamos teoremas de existência e unicidade de solução para o problema de valor inicial. Mais precisamente, dados uma função generalizada temperada $F$ (ou generalizada temperada na segunda variável), um número real $t_{0}$ e um vetor generalizado $x_{0}$, determinamos condições para que exista uma (ou somente uma) aplicação generalizada ou generalizada temperada $u$ satisfazendo:

$$
\frac{d u}{d t}=F \circ(i d, u) \quad \text { e } \quad u\left(t_{0}\right)=x_{0}
$$

Na seção 3.2 comentamos uma específica equação de Euler-Lagrange. Nessa seção além de utilizarmos os resultados da seção anterior, obtivemos outras condições para existência de solução para a referida equação. Finalizamos o capítulo, e por conseguinte o trabalho, com um estudo sobre solução para frente de sistemas autônomos.

No final desta Dissertação há um índice de símbolos e notações e as referências bi- 
bliográficas. Nessa última, além dos artigos que serviram de apoio para o desenvolvimento do nosso trabalho, listamos outros que mostram algumas aplicações da Teoria das Funções Generalizadas. Convém destacar que sempre que considerarmos um conjunto aberto ficará implícito que ele é não vazio.

São Paulo, fevereiro de 2008.

Sávio Mendes França 


\section{Capítulo 1}

\section{Algumas álgebras de Colombeau e suas propriedades}

Neste capítulo, temos por objetivo apresentar algumas das álgebras de Colombeau que, em particular, serão utilizadas nos capítulos que virão a seguir. Dentre essas álgebras destacamos as álgebras das aplicações generalizadas, das aplicações generalizadas temperadas e a das aplicações generalizadas temperadas na segunda variável, pois é com essas álgebras que a parte principal do trabalho é desenvolvida.

\section{$1.1 \quad$ A álgebra $\bar{R}$}

Antes de definirmos a álgebra dos números reais generalizados, iremos fixar algumas notações.

- $\mathbf{I}:=] 0,1]$;

- $\left.\left.\mathbf{I}_{\eta}:=\right] 0, \eta\right]$, para todo $\eta \in \mathbf{I}$ 
- $\left(a_{\varepsilon}\right)_{\varepsilon}$ denota um elemento de $A^{\mathbf{I}}$, onde $A$ é um conjunto não vazio.

Definição 1.1.1 Definimos

$$
\begin{aligned}
\mathcal{E}_{M}(\mathbb{R}) & :=\left\{\left(r_{\varepsilon}\right)_{\varepsilon} \in \mathbb{R}^{\mathbf{I}} \mid \exists N \in \mathbb{N}, \exists c>0 e \exists \eta \in \mathbf{I}, \text { tais que }\left|r_{\varepsilon}\right| \leq c \varepsilon^{-N}, \quad \forall \varepsilon \in \mathbf{I}_{\eta}\right\} \\
\mathcal{N}(\mathbb{R}) & :=\left\{\left(r_{\varepsilon}\right)_{\varepsilon} \in \mathbb{R}^{\mathbf{I}} \mid \forall m \in \mathbb{N}, \exists c>0 e \exists \eta \in \mathbf{I}, \text { tais que }\left|r_{\varepsilon}\right| \leq c \varepsilon^{m}, \quad \forall \varepsilon \in \mathbf{I}_{\eta}\right\} .
\end{aligned}
$$

Um elemento de $\mathcal{E}_{M}(\mathbb{R})$ é chamado elemento moderado e um elemento de $\mathcal{N}(\mathbb{R})$ é chamado elemento nulo.

Decorre diretamente da Definição 1.1.1 que $\mathcal{E}_{M}(\mathbb{R})$ é um anel e que $\mathcal{N}(\mathbb{R})$ é um ideal de $\mathcal{E}_{M}(\mathbb{R})$. Assim podemos apresentar a seguinte definição.

Definição 1.1.2 A álgebra quociente

$$
\overline{\mathbb{R}}:=\mathcal{E}_{M}(\mathbb{R}) / \mathcal{N}(\mathbb{R}),
$$

é chamada de álgebra dos números reais generalizados.

Um elemento de $\overline{\mathbb{R}}$ é chamado número real generalizado.

A todo elemento $\left(r_{\varepsilon}\right)_{\varepsilon} \in \mathcal{E}_{M}(\mathbb{R})$ está associado um único elemento $r \in \overline{\mathbb{R}}$ da seguinte forma

$$
r:=\left[\left(r_{\varepsilon}\right)_{\varepsilon}\right]=\left(r_{\varepsilon}\right)_{\varepsilon}+\mathcal{N}(\mathbb{R}) .
$$

Nesse caso, dizemos que $\left(r_{\varepsilon}\right)_{\varepsilon}$ é um representante de $r \in \overline{\mathbb{R}}$.

É fácil verificar que a aplicação

$$
\begin{aligned}
i_{R}: \mathbb{R} & \longrightarrow \overline{\mathbb{R}} \\
c & \longmapsto\left[(c)_{\varepsilon}\right]
\end{aligned}
$$


é um homomorfismo injetivo de álgebras. Devido a esse fato podemos identificar $\mathbb{R}$ com $i_{R}(\mathbb{R})$, e assim escrever $\mathbb{R} \subset \overline{\mathbb{R}}$.

Convém notar o seguinte.

Observação 1.1.3 O anel $\overline{\mathbb{R}}$ é um anel comutativo com unidade $i(1)=\left[(1)_{\varepsilon}\right]$ mas não é um anel de integridade.

De fato, a primeira afirmação é imediata. Para a segunda, considere $\left(r_{\varepsilon}\right)_{\varepsilon}$ e $\left(s_{\varepsilon}\right)_{\varepsilon}$ definidos por

$r_{\varepsilon}:=\left\{\begin{array}{ll}0 & \text { se } \varepsilon=\frac{1}{n}, \text { para algum } n \in \mathbb{N}^{*}, \\ 1 & \text { caso contrário }\end{array} ; s_{\varepsilon}:=\left\{\begin{array}{ll}1 & \text { se } \varepsilon=\frac{1}{n}, \text { para algum } n \in \mathbb{N}^{*}, \\ 0 & \text { caso contrário }\end{array}\right.\right.$.

Então $\left(r_{\varepsilon}\right)_{\varepsilon},\left(s_{\varepsilon}\right)_{\varepsilon} \in \mathcal{E}_{M}(\mathbb{R}) \backslash \mathcal{N}(\mathbb{R})$ e $r_{\varepsilon} . s_{\varepsilon}=0$, para todo $\varepsilon \in \mathbf{I}$. Logo, $r:=\left[\left(r_{\varepsilon}\right)_{\varepsilon}\right] \neq 0$, $s:=\left[\left(s_{\varepsilon}\right)_{\varepsilon}\right] \neq 0$ e $r . s=0$.

De modo análogo, substituindo $\mathbb{R}$ por $\mathbb{C}$ na Definição 1.1 .1 e na Definição 1.1.2, define-se a álgebra do números complexos generalizados, denotada por $\overline{\mathbb{C}}$.

D. Scarpalezos ([Sca-1] e [Sca-2]) definiu uma ultramétrica sobre $\bar{K}$ (onde $K=\mathbb{R}$ ou $\mathbb{C}$ ) de modo que, com a topologia induzida por essa ultramétrica, tem-se que $\overline{\mathbb{K}}$ é um anel topológico completo. A partir dessa topologia, J. Aragona e S. O. Juriaans ([AJ]) obtiveram propriedades da estrutura algébrica e topológica de $\overline{\mathbb{K}}$ e estabeleceram importantes conexões entre essas estruturas. Uma prova detalhada de alguns desses resultados podem ser encontrados em [Sil-A] e [Vei]. Em [Vei] é utilizada a topologia de D. Scarpalezos mas com a abordagem feita por J. Aragona, R. Fernandez e S. O. Juriaans ([AFJ-3]). 


\section{$1.2 \quad$ A álgebra $\mathcal{G}\left(\Omega ; \mathbb{R}^{m}\right)$}

O objetivo desta seção é relembrar a construção da álgebra $\mathcal{G}\left(\Omega ; \mathbb{R}^{m}\right)$ e estudar algumas de suas propriedades que serão úteis neste trabalho. Em particular, destacamos o Teorema de Nulidade em $\mathcal{E}_{M}\left[\Omega ; \mathbb{R}^{m}\right]$ (Teorema 1.2.5). Esse teorema foi apresentado por M. Grosser, M. Kunzinger, M. Oberguggenberger e R. Steinbauer em [GKOS].

Definição 1.2.1 Seja $\Omega$ um aberto de $\mathbb{R}^{n}$. Definimos

$$
\begin{aligned}
& \mathcal{E}[\Omega ; \mathbb{R}]:=\left(\mathscr{C}^{\infty}(\Omega ; \mathbb{R})\right)^{\mathbf{I}} ; \\
& \mathcal{E}_{M}[\Omega ; \mathbb{R}]:=\left\{\left(u_{\varepsilon}\right)_{\varepsilon} \in \mathcal{E}[\Omega ; \mathbb{R}] \mid \forall K \subset \subset \Omega, \forall \alpha \in \mathbb{N}^{n}, \exists N \in \mathbb{N}, \exists c>0 e \exists \eta \in \mathbf{I},\right. \\
& \text { tais que } \left.\left|\partial^{\alpha} u_{\varepsilon}(x)\right| \leq c \varepsilon^{-N}, \quad \forall(\varepsilon, x) \in \mathbf{I}_{\eta} \times K\right\} ; \\
& \mathcal{N}[\Omega ; \mathbb{R}]:=\left\{\left(u_{\varepsilon}\right)_{\varepsilon} \in \mathcal{E}[\Omega ; \mathbb{R}] \mid \forall K \subset \subset \Omega, \forall \alpha \in \mathbb{N}^{n}, \forall r \in \mathbb{N}, \exists c>0 e \exists \eta \in \mathbf{I},\right. \\
& \text { tais que } \left.\left|\partial^{\alpha} u_{\varepsilon}(x)\right| \leq c \varepsilon^{r}, \quad \forall(\varepsilon, x) \in \mathbf{I}_{\eta} \times K\right\} .
\end{aligned}
$$

Um elemento de $\mathcal{E}_{M}[\Omega ; \mathbb{R}]$ é chamado de função moderada em $\Omega$ a valores em $\mathbb{R}$ e um elemento de $\mathcal{N}[\Omega ; \mathbb{R}]$ é chamado de função nula em $\Omega$ a valores em $\mathbb{R}$.

O conjunto $\mathcal{E}_{M}[\Omega ; \mathbb{R}]$ munido das operações usuais de soma e produto de funções e produto de número real por função é uma $\mathbb{R}$-álgebra. O conjunto $\mathcal{N}[\Omega ; \mathbb{R}]$ é um ideal de $\mathcal{E}_{M}[\Omega ; \mathbb{R}]$. Assim, pode-se definir a seguinte álgebra.

Definição 1.2.2 Seja $\Omega$ um aberto de $\mathbb{R}^{n}$. A álgebra quociente

$$
\mathcal{G}(\Omega ; \mathbb{R}):=\mathcal{E}_{M}[\Omega ; \mathbb{R}] / \mathcal{N}[\Omega ; \mathbb{R}],
$$

é chamada de álgebra das funções generalizadas de Colombeau. 
Um elemento de $\mathcal{G}(\Omega ; \mathbb{R})$ é chamado função generalizada em $\Omega$ a valores em $\mathbb{R}$.

Definição 1.2.3 Seja $\Omega$ um aberto de $\mathbb{R}^{n}$ e $m \in \mathbb{N}$ com $m \geq 2$. Definimos

$$
\mathcal{G}\left(\Omega ; \mathbb{R}^{m}\right):=\left(\mathcal{E}_{M}[\Omega ; \mathbb{R}]\right)^{m} /(\mathcal{N}[\Omega ; \mathbb{R}])^{m} .
$$

Um elemento de $\mathcal{G}\left(\Omega ; \mathbb{R}^{m}\right)$ é chamado aplicação generalizada em $\Omega$ a valores em $\mathbb{R}^{m}$ e $\mathcal{G}\left(\Omega ; \mathbb{R}^{m}\right)$ é chamada de álgebra das aplicações generalizadas de Colombeau.

Para facilitar a escrita escreveremos $\mathcal{E}_{M}\left[\Omega ; \mathbb{R}^{m}\right]$ e $\mathcal{N}\left[\Omega ; \mathbb{R}^{m}\right]$ em vez de $\left(\mathcal{E}_{M}[\Omega ; \mathbb{R}]\right)^{m}$ e $(\mathcal{N}[\Omega ; \mathbb{R}])^{m}$, respectivamente.

É fácil verificar que a aplicação

$$
\begin{aligned}
(\mathcal{G}(\Omega ; \mathbb{R}))^{m} & \longrightarrow \mathcal{G}\left(\Omega ; \mathbb{R}^{m}\right) \\
\left(u_{1_{\varepsilon}}+\mathcal{N}[\Omega ; \mathbb{R}], \cdots, u_{m_{\varepsilon}}+\mathcal{N}[\Omega ; \mathbb{R}]\right) & \longmapsto\left(u_{1_{\varepsilon}}, \cdots, u_{m_{\varepsilon}}\right)+\mathcal{N}\left[\Omega ; \mathbb{R}^{m}\right],
\end{aligned}
$$

é um isomorfismo de álgebras. Assim, para cada $m \in \mathbb{N}^{*}$, podemos identificar $\mathcal{G}\left(\Omega ; \mathbb{R}^{m}\right)$ $\operatorname{com}(\mathcal{G}(\Omega ; \mathbb{R}))^{m}$. Além disso, convém observar o seguinte.

Observação 1.2.4 Sejam $\Omega$ um aberto de $\mathbb{R}^{n}$ e $\left(u_{\varepsilon}\right)_{\varepsilon} \in(\mathcal{E}[\Omega ; \mathbb{R}])^{m}$. Tem-se

1. $\left(u_{\varepsilon}\right)_{\varepsilon} \in \mathcal{E}_{M}\left[\Omega ; \mathbb{R}^{m}\right]$ se, e somente se, para quaisquer $K \subset \subset \Omega$ e $\alpha \in \mathbb{N}^{n}$, existem $N \in \mathbb{N}, c>0$ e $\eta \in \mathbf{I}$ tais que

$$
\left|\partial^{\alpha} u_{\varepsilon}(x)\right| \leq c \varepsilon^{-N}, \quad \forall(\varepsilon, x) \in \mathbf{I}_{\eta} \times K ;
$$

2. $\left(u_{\varepsilon}\right)_{\varepsilon} \in \mathcal{N}\left[\Omega ; \mathbb{R}^{m}\right]$ se, e somente se, para quaisquer $K \subset \subset \Omega, \alpha \in \mathbb{N}^{n}$ e $r \in \mathbb{N}$, existem $c>0$ e $\eta \in \mathbf{I}$ tais que

$$
\left|\partial^{\alpha} u_{\varepsilon}(x)\right| \leq c \varepsilon^{r}, \quad \forall(\varepsilon, x) \in \mathbf{I}_{\eta} \times K .
$$


Observe que, se $\Omega$ é um aberto de $\mathbb{R}^{n}$, então todo $\left(u_{\varepsilon}\right)_{\varepsilon} \in \mathcal{E}_{M}\left[\Omega ; \mathbb{R}^{m}\right]$ está associado a um único elemento $u \in \mathcal{G}\left(\Omega ; \mathbb{R}^{m}\right)$ da seguinte forma

$$
u:=\left[\left(u_{\varepsilon}\right)_{\varepsilon}\right]=\left(u_{\varepsilon}\right)_{\varepsilon}+\mathcal{N}\left[\Omega ; \mathbb{R}^{m}\right]
$$

Nesse caso, dizemos que $\left(u_{\varepsilon}\right)_{\varepsilon} \in \mathcal{E}_{M}\left[\Omega ; \mathbb{R}^{m}\right]$ é um representante de $u \in \mathcal{G}\left(\Omega ; \mathbb{R}^{m}\right)$.

Note também que, se $\Omega$ é um aberto de $\mathbb{R}^{n}$ e $f \in \mathscr{C}^{\infty}\left(\Omega ; \mathbb{R}^{m}\right)$, então $(f)_{\varepsilon} \in \mathcal{E}_{M}\left[\Omega ; \mathbb{R}^{m}\right]$. Assim, é fácil verificar que a aplicação

$$
\begin{aligned}
i_{\mathscr{C} \infty}\left(\Omega ; R^{m}\right): \mathscr{C}^{\infty}\left(\Omega ; \mathbb{R}^{m}\right) & \longrightarrow \mathcal{G}\left(\Omega ; \mathbb{R}^{m}\right) \\
f & \longmapsto(f)_{\varepsilon}+\mathcal{N}\left[\Omega ; \mathbb{R}^{m}\right]
\end{aligned}
$$

é um homomorfismo injetivo de álgebras. Devido a esse fato podemos identificar $\mathscr{C}^{\infty}\left(\Omega ; \mathbb{R}^{m}\right) \operatorname{com} i_{\mathscr{C} \infty}\left(\Omega ; R^{m}\right)\left(\mathscr{C}^{\infty}\left(\Omega ; \mathbb{R}^{m}\right)\right)$ e assim escrever $\mathscr{C}^{\infty}\left(\Omega ; \mathbb{R}^{m}\right) \subset \mathcal{G}\left(\Omega ; \mathbb{R}^{m}\right)$.

A seguir, apresentaremos o Teorema de Nulidade para funções em $\mathcal{E}_{M}[\Omega ; \mathbb{R}]$ encontrado em [GKOS]. Devido a sua importância, faremos a demonstração na íntegra.

Teorema 1.2.5 (Teorema de Nulidade em $\mathcal{E}_{M}\left[\Omega ; \mathbb{R}^{m}\right]$ ) Sejam $\Omega$ um aberto de $\mathbb{R}^{n}$ e $\left(u_{\varepsilon}\right)_{\varepsilon} \in \mathcal{E}_{M}\left[\Omega ; \mathbb{R}^{m}\right]$. São equivalentes as seguintes afirmações:

(a) $\left(u_{\varepsilon}\right)_{\varepsilon} \in \mathcal{N}\left[\Omega ; \mathbb{R}^{m}\right]$

(b) $\left(u_{\varepsilon}\right)_{\varepsilon}$ satisfaz a $\mathcal{N}$-estimativa de ordem zero, isto é, $\forall K \subset \subset \Omega, \forall r \in \mathbb{N}, \exists c>0 e \exists \eta \in \mathbf{I}$ tais que $\left|u_{\varepsilon}(x)\right| \leq c \varepsilon^{r}, \quad \forall(\varepsilon, x) \in \mathbf{I}_{\eta} \times K$

Demonstração: Decorre diretamente da definição de $\mathcal{N}\left[\Omega ; \mathbb{R}^{m}\right]$ que $(a)$ implica $(b)$.

Para provarmos que $(b)$ implica $(a)$ notemos que basta termos a implicação no caso $m=1$.

Suponha $m=1$. 
Provaremos por indução sobre $|\alpha|$, onde $\alpha \in \mathbb{N}^{n}$, que

$\forall K \subset \subset \Omega, \forall r \in \mathbb{N}, \exists c>0$ e $\exists \eta \in \mathbf{I}$ tais que $\left|\partial^{\alpha} u_{\varepsilon}(x)\right| \leq c \varepsilon^{r}, \quad \forall(\varepsilon, x) \in \mathbf{I}_{\eta} \times K$

É claro que, se $|\alpha|=0$, então (1.2), por (b), é verdadeira.

Seja $\alpha=\left(\alpha_{1}, \cdots, \alpha_{n}\right) \in \mathbb{N}^{n} \operatorname{com}|\alpha|>0$ e suponha (1.2) verdadeira para todo $\gamma \in \mathbb{N}^{n}$ com $|\gamma|=|\alpha|-1$. Note que existe $1 \leq j \leq n$ tal que $\alpha_{j} \neq 0$, e assim

$$
\partial^{\alpha} u_{\varepsilon}=\frac{\partial}{\partial x_{j}}\left(\frac{\partial^{|\alpha|-1} u_{\varepsilon}}{\partial x_{1}^{\alpha_{1}} \cdots \partial x_{j}^{\alpha_{j}-1} \cdots \partial x_{n}^{\alpha_{n}}}\right)=\frac{\partial v_{\varepsilon}}{\partial x_{j}}
$$

onde $v_{\varepsilon}:=\left(\frac{\partial^{|\alpha|-1} u_{\varepsilon}}{\partial x_{1}^{\alpha_{1}} \cdots \partial x_{j}^{\alpha_{j}-1} \cdots \partial x_{n}^{\alpha_{n}}}\right) \in \mathcal{E}_{M}[\Omega ; \mathbb{R}]$, para todo $\varepsilon \in \mathbf{I}$. Logo, pela hipótese de indução,

$\forall K_{1} \subset \subset \Omega, \forall s \in \mathbb{N}, \exists c>0$ e $\exists \eta \in \mathbf{I}$ tais que $\left|v_{\varepsilon}(x)\right| \leq c \varepsilon^{s}, \quad \forall(\varepsilon, x) \in \mathbf{I}_{\eta} \times K_{1}$

Sejam $K \subset \subset \Omega$ e $r \in \mathbb{N}$. Para facilitar a escrita utilizaremos as seguintes notações:

$$
\partial_{i}:=\frac{\partial}{\partial x_{i}} \quad ; \quad \partial_{i}^{2}:=\frac{\partial^{2}}{\partial x_{i}^{2}}
$$

onde $x_{i}$ é a i-ésima coordenada de um ponto arbitrário de $\mathbb{R}^{n}$ e $1 \leq i \leq n$.

Como $K \subset \subset \Omega$, existe $V$ aberto de $\Omega$ tal que $K \subset V \subset \bar{V} \subset \subset \Omega$. Considere $\nu=\frac{1}{2} \min \{1, d(K, \Omega \backslash V)\}$ e seja

$$
L:=K+\overline{B_{\nu}(0)}=\left\{x+y \mid(x, y) \in K \times \overline{B_{\nu}(0)}\right\}
$$

Então $K \subset L \subset \subset \Omega$.

De $\left(v_{\varepsilon}\right)_{\varepsilon} \in \mathcal{E}_{M}[\Omega ; \mathbb{R}]$, existem $N \in \mathbb{N}, c_{1}>0$ e $\eta_{1} \in \mathbf{I}$ tais que

$$
\left|\partial_{i}^{2} v_{\varepsilon}(x)\right| \leq c_{1} \varepsilon^{-N}, \quad \forall(\varepsilon, x) \in \mathbf{I}_{\eta_{1}} \times L
$$

De (1.3), para $K_{1}:=L$ e $s:=2 r+N+1$, existem $c_{2}>c_{1}$ e $\eta_{2} \in \mathbf{I}$ com $\eta_{2}<\eta_{1}$ tais que

$$
\left|v_{\varepsilon}(x)\right| \leq c_{2} \varepsilon^{2 r+N+1}, \quad \forall(\varepsilon, x) \in \mathbf{I}_{\eta_{2}} \times L .
$$


Seja $\eta:=\min \left\{\eta_{2}, \nu\right\}$ e note que, para todo $(\varepsilon, x) \in \mathbf{I}_{\eta} \times K$, o segmento de extremidades $x$ e $x+\varepsilon^{r+N+1} e_{i}$ está contido em $L$, onde $e_{i}$ é o i-ésimo elemento da base canônica de $\mathbb{R}^{n}$, pois os elementos desse segmento são da forma $x+t \varepsilon^{r+N+1} e_{i}$ para $t \in[0,1]$. Dessa forma, usando o Polinômio de Taylor de ordem 1, temos que, para $(\varepsilon, x) \in \mathbf{I}_{\eta} \times K$, existe $y=x+\sigma \varepsilon^{r+N+1} e_{i} \in L$, para algum $\left.\sigma \in\right] 0,1[$, tal que

$$
v_{\varepsilon}\left(x+\varepsilon^{r+N+1} e_{i}\right)=v_{\varepsilon}(x)+\partial_{i} v_{\varepsilon}(x) \varepsilon^{r+N+1}+\frac{1}{2} \partial_{i}^{2} v_{\varepsilon}(y)\left(\varepsilon^{r+N+1}\right)^{2}
$$

ou seja,

$$
\partial_{i} v_{\varepsilon}(x) \varepsilon^{r+N+1}=v_{\varepsilon}\left(x+\varepsilon^{r+N+1} e_{i}\right)-v_{\varepsilon}(x)-\frac{1}{2} \partial_{i}^{2} v_{\varepsilon}(y)\left(\varepsilon^{r+N+1}\right)^{2} .
$$

Assim, de (1.4) e (1.5), temos, para $(\varepsilon, x) \in \mathbf{I}_{\eta} \times K$ que

$$
\begin{aligned}
\left|\partial_{i} v_{\varepsilon}(x)\right| & \leq\left(\left|v_{\varepsilon}\left(x+\varepsilon^{r+N+1} e_{i}\right)\right|+\left|v_{\varepsilon}(x)\right|\right) \varepsilon^{-r-N-1}+\frac{1}{2}\left|\partial_{i}^{2} v_{\varepsilon}(y)\right| \varepsilon^{r+N+1} \\
& \leq\left(c_{2} \varepsilon^{2 r+N+1}+c_{2} \varepsilon^{2 r+N+1}\right) \varepsilon^{-r-N-1}+\frac{1}{2} c_{1} \varepsilon^{-N} \varepsilon^{r+N+1} \\
& \leq 2 c_{2} \varepsilon^{r}+\frac{1}{2} c_{2} \varepsilon^{r}
\end{aligned}
$$

Logo, se $c:=\frac{5}{2} c_{2}$, temos que

$$
\left|\partial_{i} v_{\varepsilon}(x)\right| \leq c \varepsilon^{r}, \quad \forall(\varepsilon, x) \in \mathbf{I}_{\eta} \times K
$$

e $\operatorname{assim}(1.2)$ é verdadeira.

A seguir, apresentaremos a derivada de uma aplicação generalizada. Antes porém, observemos que, se $\Omega$ é um aberto de $\mathbb{R}^{n},\left(u_{\varepsilon}\right)_{\varepsilon} \in \mathcal{E}_{M}\left[\Omega ; \mathbb{R}^{m}\right], \alpha \in \mathbb{N}^{n}$ e $\left(\partial^{\alpha} u_{\varepsilon}\right)_{\varepsilon} \in \mathcal{E}\left[\Omega ; \mathbb{R}^{m}\right]$ é definida por

$$
\partial^{\alpha} u_{\varepsilon}: x \in \Omega \longmapsto\left(\partial^{\alpha} u_{\varepsilon}\right)(x) \in \mathbb{R}^{m}
$$

então $\left(\partial^{\alpha} u_{\varepsilon}\right)_{\varepsilon} \in \mathcal{E}_{M}\left[\Omega ; \mathbb{R}^{m}\right]$. Assim, das propriedades lineares das derivadas, decorre que a aplicação

$$
\begin{aligned}
\partial^{\alpha}: \mathcal{E}_{M}\left[\Omega ; \mathbb{R}^{m}\right] & \longrightarrow \mathcal{E}_{M}\left[\Omega ; \mathbb{R}^{m}\right] \\
\left(u_{\varepsilon}\right)_{\varepsilon} & \longmapsto\left(\partial^{\alpha} u_{\varepsilon}\right)_{\varepsilon}
\end{aligned}
$$


é um homomorfismo de $\mathbb{R}$-espaços vetoriais. Note ainda que $\partial^{\alpha}\left(\mathcal{E}_{M}\left[\Omega ; \mathbb{R}^{m}\right]\right) \subset \mathcal{E}_{M}\left[\Omega ; \mathbb{R}^{m}\right]$ e $\partial^{\alpha}\left(\mathcal{N}\left[\Omega ; \mathbb{R}^{m}\right]\right) \subset \mathcal{N}\left[\Omega ; \mathbb{R}^{m}\right]$. Portanto, do Teorema do Homomorfismo, existe uma única aplicação $\mathbb{R}$-linear $\partial^{\alpha}: \mathcal{G}\left(\Omega ; \mathbb{R}^{m}\right) \longrightarrow \mathcal{G}\left(\Omega ; \mathbb{R}^{m}\right)$ tal que o diagrama

$$
\begin{array}{ccc}
\mathcal{E}_{M}\left[\Omega ; \mathbb{R}^{m}\right] & \stackrel{\partial^{\alpha}}{\longrightarrow} & \mathcal{E}_{M}\left[\Omega ; \mathbb{R}^{m}\right] \\
j \downarrow & j \downarrow \\
\mathcal{G}\left(\Omega ; \mathbb{R}^{m}\right) & \stackrel{\partial^{\alpha}}{\longrightarrow} & \mathcal{G}\left(\Omega ; \mathbb{R}^{m}\right)
\end{array}
$$

comuta, onde $j:\left(u_{\varepsilon}\right)_{\varepsilon} \in \mathcal{E}_{M}\left[\Omega ; \mathbb{R}^{m}\right] \longmapsto\left(u_{\varepsilon}\right)_{\varepsilon}+\mathcal{N}\left[\Omega ; \mathbb{R}^{m}\right]$. A partir desse fato, temos a seguinte definição.

Definição 1.2.6 Sejam $\Omega$ um aberto de $\mathbb{R}^{n}, u:=\left[\left(u_{\varepsilon}\right)_{\varepsilon}\right] \in \mathcal{G}\left(\Omega ; \mathbb{R}^{m}\right)$ e $\alpha \in \mathbb{N}^{n}$. Chamamos de derivada parcial de ordem $\alpha$ de $u$, o elemento $\partial^{\alpha} u:=\left[\left(\partial^{\alpha} u_{\varepsilon}\right)_{\varepsilon}\right] \in \mathcal{G}\left(\Omega ; \mathbb{R}^{m}\right)$.

A Definição 1.2.6 é compatível com a fórmula de Leibniz, ou seja,

se $u, v \in \mathcal{G}(\Omega ; \mathbb{R})$ e $\alpha \in \mathbb{N}^{n}$, então tem-se

$$
\partial^{\alpha}(u v)=\sum_{0 \leq \beta \leq \alpha}\left(\begin{array}{l}
\alpha \\
\beta
\end{array}\right)\left(\partial^{\beta} u\right)\left(\partial^{\alpha-\beta} v\right)
$$

Definição 1.2.7 Sejam $\Omega$ um aberto de $\mathbb{R}^{n}$ e $u:=\left[\left(u_{\varepsilon}\right)_{\varepsilon}\right] \in \mathcal{G}(\Omega ; \mathbb{R})$. Chamamos de gradiente de u e denotamos por $\nabla u$ a função generalizada

$$
\nabla u:=\left(\frac{\partial u_{\varepsilon}}{\partial x_{1}}, \cdots, \frac{\partial u_{\varepsilon}}{\partial x_{n}}\right)+\mathcal{N}\left[\Omega ; \mathbb{R}^{n}\right] .
$$

onde $x_{i}$ é a i-ésima coordenada de um ponto arbitrário de $\mathbb{R}^{n}$ e $1 \leq i \leq n$.

É claro que, se $u \in \mathcal{G}(\Omega ; \mathbb{R})$, então $\nabla u \in \mathcal{G}\left(\Omega ; \mathbb{R}^{n}\right)$.

Um resultado importante, e cuja prova pode ser encontrada em [Vei], é o seguinte: 
Seja $\Omega$ um aberto conexo de $\mathbb{R}^{n}$ e $u \in \mathcal{G}(\Omega ; \mathbb{R})$. Tem-se que $\nabla u \equiv 0$ se, e somente se existe $c \in \mathbb{R}$ tal que $u=(x \in \Omega \longmapsto c)_{\varepsilon}+\mathcal{N}[\Omega ; \mathbb{R}]$.

Apresentaremos, a seguir, o conceito de função composta no contexto das funções generalizadas de Colombeau. Antes porém, convém observar que, se $\Omega$ é um aberto de $\mathbb{R}^{n}$, $\Omega^{\prime}$ um aberto de $\mathbb{R}^{p}$ e $\left(u_{\varepsilon}\right)_{\varepsilon} \in \mathcal{E}_{M}\left[\Omega ; \mathbb{R}^{p}\right]$ e $\left(v_{\varepsilon}\right)_{\varepsilon} \in \mathcal{E}_{M}\left[\Omega^{\prime} ; \mathbb{R}^{s}\right]$ são tais que $u_{\varepsilon}(\Omega) \subset \Omega^{\prime}$ para todo $\varepsilon \in \mathbf{I}$, então a função $\left(v_{\varepsilon} \circ u_{\varepsilon}\right)_{\varepsilon}$ pode não pertencer a $\mathcal{E}\left[\Omega ; \mathbb{R}^{s}\right]$. Por exemplo, se $\left(v_{\varepsilon}\right)_{\varepsilon}$ e $\left(u_{\varepsilon}\right)_{\varepsilon}$ são definidas por $u_{\varepsilon}(x)=\frac{x^{2}}{\varepsilon}$ e $\quad v_{\varepsilon}(x)=e^{x}$ para $(\varepsilon, x) \in \mathbf{I} \times \mathbb{R}$, então $\left(v_{\varepsilon}\right)_{\varepsilon},\left(u_{\varepsilon}\right)_{\varepsilon} \in \mathcal{E}_{M}[\mathbb{R} ; \mathbb{R}]$ mas $\left(v_{\varepsilon} \circ u_{\varepsilon}\right)_{\varepsilon} \notin \mathcal{E}_{M}[\mathbb{R} ; \mathbb{R}]$. Para contornar esse tipo de problema, J. Aragona introduziu o conjunto $\mathcal{G}_{*}\left(\Omega ; \Omega^{\prime}\right)$. Mais precisamente,

Definição 1.2.8 Sejam $\Omega$ um aberto de $\mathbb{R}^{n}$ e $\Omega^{\prime}$ um aberto de $\mathbb{R}^{p}$. Denotamos por $\mathcal{G}_{*}\left(\Omega ; \Omega^{\prime}\right)$ o conjunto das aplicações generalizadas $u \in \mathcal{G}\left(\Omega ; \mathbb{R}^{p}\right)$ tais que existe $\left(u_{\varepsilon}\right)_{\varepsilon}$ representante de u satisfazendo:

$$
\forall K \subset \subset \Omega, \exists K^{\prime} \subset \subset \Omega^{\prime} e \exists \eta \in \mathbf{I} \text { tais que } u_{\varepsilon}(K) \subset K^{\prime}, \quad \forall \varepsilon \in \mathbf{I}_{\eta} .
$$

Note que, $\mathcal{G}_{*}(\Omega ; \mathbb{R})$ é uma $\mathbb{R}$-álgebra. Além disso, prova-se que, se $u \in \mathcal{G}_{*}\left(\Omega ; \Omega^{\prime}\right)$, então todo representante de $u$ satisfaz (1.6). Mais propriedades do conjunto $\mathcal{G}_{*}\left(\Omega ; \Omega^{\prime}\right)$ podem ser encontradas em [Fer].

Definição 1.2.9 Seja $\Omega$ um aberto de $\mathbb{R}^{n}, \quad \Omega^{\prime}$ um aberto de $\mathbb{R}^{p}, \quad u \in \mathcal{G}_{*}\left(\Omega ; \Omega^{\prime}\right)$ e $v \in \mathcal{G}\left(\Omega^{\prime} ; \mathbb{R}^{s}\right)$. A função composta de u e $v$, denotada por $v \circ u$, e cuja existência é provada em $[\mathrm{Fer}]$, é a função de $\mathcal{G}\left(\Omega ; \mathbb{R}^{s}\right)$ que tem a seguinte propriedade:

se $\left(u_{\varepsilon}\right)_{\varepsilon}$ é um representante de $u,\left(v_{\varepsilon}\right)_{\varepsilon}$ é um representante de $v, \mathcal{K}=\left(K_{j}\right)_{j \in N}$ é uma seqüência exaustiva de compactos para $\Omega$, isto é,

$$
\bigcup_{j \in N} K_{j}=\Omega, K_{j} \subset K_{j+1}^{\circ}, \quad \forall j \in \mathbb{N}
$$


e se $\left(\eta_{j}\right)_{j \in N}$ é uma seqüência em $\mathbf{I}$ tal que

$$
\begin{aligned}
& \eta_{j}>\eta_{j+1}, \quad \forall j \in \mathbb{N} ; \\
& \overline{\left\{u_{\varepsilon}(x):(\varepsilon, x) \in \mathbf{I}_{\eta_{j}} \times K_{j}\right\}} \subset \subset \Omega^{\prime}, \quad \forall j \in \mathbb{N} ;
\end{aligned}
$$

então existe um representante $\left(\Theta_{\mathcal{K}, u_{\varepsilon}, v_{\varepsilon}}\right)_{\varepsilon}$ de $v \circ u$ de modo que

$$
\left(\left.\Theta_{\mathcal{K}, u_{\varepsilon}, v_{\varepsilon}}\right|_{\stackrel{\circ}{K}_{j}}\right)_{\varepsilon}-\left(h_{j \varepsilon}\right)_{\varepsilon} \in \mathcal{N}\left[\stackrel{\circ}{K_{j}} ; \mathbb{R}^{s}\right], \quad \forall j \in \mathbb{N}
$$

onde

$$
h_{j \varepsilon}(x)=\left\{\begin{array}{lll}
v_{\varepsilon}\left(u_{\varepsilon}(x)\right) & \text { se } & (\varepsilon, x) \in \mathbf{I}_{\eta_{j}} \times \stackrel{\circ}{K_{j}} \\
v_{\varepsilon}\left(u_{\eta_{j} / 2}(x)\right) & \text { se } & (\varepsilon, x) \in\left[\eta_{j}, 1\right] \times \stackrel{\circ}{K_{j}}
\end{array} .\right.
$$

Finalizamos esta seção com a definição de valor pontual de uma função generalizada. Para tal observe que, se $\Omega$ um aberto de $\mathbb{R}^{n}, t_{0} \in \Omega, u \in \mathcal{G}(\Omega ; \mathbb{R})$ e se $\left(u_{\varepsilon}\right)_{\varepsilon}$ e $\left(v_{\varepsilon}\right)_{\varepsilon}$ são representantes de $u$, então $\left(u_{\varepsilon}\left(t_{0}\right)-v_{\varepsilon}\left(t_{0}\right)\right) \in \mathcal{N}(\mathbb{R})$. A partir desse fato, tem-se a seguinte definição.

Definição 1.2.10 Sejam $u \in \mathcal{G}\left(\Omega ; \mathbb{R}^{m}\right)$ e $t_{0} \in \Omega$. Definimos o valor pontual de $u$ em $t_{0}$ por

$$
u\left(t_{0}\right):=\left(u_{\varepsilon}\left(t_{0}\right)\right)_{\varepsilon}+\mathcal{N}(\mathbb{R})
$$

onde $\left(u_{\varepsilon}\right)_{\varepsilon}$ é um representante qualquer de $u$

É importante ressaltar que existe $u \in \mathcal{G}(\Omega ; \mathbb{R})$ com $u \neq 0$ e tal que $u\left(t_{0}\right)=0$, para todo $t_{0} \in \Omega([$ Vei]). Recentemente, M. Kunzinger e M. Oberguggenberger introduziram o conjunto $\widetilde{\Omega}_{c}$ e um novo conceito de valor pontual generalizado de modo que uma função é nula se, e somente se, o seu valor pontual generalizado é nulo. J. Aragona, R. Fernandez e S. O. Juriaans, a partir desse novo conceito e da topologia introduzida por D. Scarpalezos ([Sca-1] e [Sca-2]), introduzem uma noção de diferenciabilidade para funções definidas em abertos de $\overline{\mathbb{R}}^{n}$ (respectivamente $\overline{\mathbb{C}}$ ) e a valores em $\overline{\mathbb{R}}^{m}$ (resp. $\overline{\mathbb{C}}$ ) ([AFJ-1]). Uma prova 
detalhada de alguns dos resultados apresentados em [AFJ-1] pode ser encontrada em [Vei].

\subsection{A álgebra $\mathcal{G}_{\tau}\left(\Omega ; \mathbb{R}^{m}\right)$}

Nesta seção, apresentaremos a álgebra $\mathcal{G}_{\tau}\left(\Omega ; \mathbb{R}^{m}\right)$, isto é, a álgebra das aplicações generalizadas temperadas de Colombeau. Destacamos o Teorema de Nulidade em $\mathcal{E}_{\tau}\left[\Omega ; \mathbb{R}^{m}\right]$ (Teorema 1.3.7) que, além de facilitar a prova de alguns dos resultados, é uma extensão do encontrado em [GKOS].

Definição 1.3.1 Seja $\Omega$ um aberto de $\mathbb{R}^{n}$. Definimos

$$
\begin{aligned}
\mathcal{E}_{\tau}[\Omega ; \mathbb{R}]:= & \left\{\left(u_{\varepsilon}\right)_{\varepsilon} \in \mathcal{E}[\Omega ; \mathbb{R}] \mid \forall \alpha \in \mathbb{N}^{n}, \exists N \in \mathbb{N}, \exists c>0 \text { e } \exists \eta \in \mathbf{I}\right. \text { tais que } \\
& \left.\left|\partial^{\alpha} u_{\varepsilon}(x)\right| \leq c(1+|x|)^{N} \varepsilon^{-N}, \quad \forall(\varepsilon, x) \in \mathbf{I}_{\eta} \times \Omega\right\} ; \\
\mathcal{N}_{\tau}[\Omega ; \mathbb{R}]:=\{ & \left(u_{\varepsilon}\right)_{\varepsilon} \in \mathcal{E}[\Omega ; \mathbb{R}] \mid \forall \alpha \in \mathbb{N}^{n}, \exists N \in \mathbb{N}, \forall r \in \mathbb{N}, \exists c>0 \text { e } \exists \eta \in \mathbf{I} \\
& \text { tais que } \left.\left|\partial^{\alpha} u_{\varepsilon}(x)\right| \leq c(1+|x|)^{N} \varepsilon^{r}, \quad \forall(\varepsilon, x) \in \mathbf{I}_{\eta} \times \Omega\right\} .
\end{aligned}
$$

Um elemento de $\mathcal{E}_{\tau}[\Omega ; \mathbb{R}]$ é chamado de função moderada temperada em $\Omega$ a valores em $\mathbb{R}$ e um elemento de $\mathcal{N}_{\tau}[\Omega ; \mathbb{R}]$ é chamado de função nula temperada em $\Omega$ a valores em $\mathbb{R}$.

O conjunto $\mathcal{E}_{\tau}[\Omega ; \mathbb{R}]$ munido das operações usuais de soma e produto de funções e produto de número real por função é uma $\mathbb{R}$-álgebra. Decorre diretamente da Definição 1.3.1 que $\mathcal{N}_{\tau}[\Omega ; \mathbb{R}]$ é um ideal de $\mathcal{E}_{\tau}[\Omega ; \mathbb{R}]$. Assim, pode-se definir o seguinte. 
Definição 1.3.2 Seja $\Omega$ um aberto de $\mathbb{R}^{n}$. A álgebra das funções generalizadas temperadas de Colombeau é definida pelo espaço quociente

$$
\mathcal{G}_{\tau}(\Omega ; \mathbb{R}):=\mathcal{E}_{\tau}[\Omega ; \mathbb{R}] / \mathcal{N}_{\tau}[\Omega ; \mathbb{R}]
$$

Um elemento de $\mathcal{G}_{\tau}(\Omega ; \mathbb{R})$ é chamado função generalizada temperada em $\Omega$ a valores em $\mathbb{R}$.

Definição 1.3.3 Sejam $\Omega$ um aberto de $\mathbb{R}^{n}$ e $m \in \mathbb{N}$ com $m \geq 2$. Definimos

$$
\mathcal{G}_{\tau}\left(\Omega ; \mathbb{R}^{m}\right):=\left(\mathcal{E}_{\tau}[\Omega ; \mathbb{R}]\right)^{m} /\left(\mathcal{N}_{\tau}[\Omega ; \mathbb{R}]\right)^{m}
$$

Um elemento de $\mathcal{G}_{\tau}\left(\Omega ; \mathbb{R}^{m}\right)$ é chamado de aplicação generalizada temperada em $\Omega$ a valores em $\mathbb{R}^{m}$. A álgebra $\mathcal{G}_{\tau}\left(\Omega ; \mathbb{R}^{m}\right)$ é chamada de álgebra das aplicações generalizadas temperadas de Colombeau.

Para facilitar a escrita escreveremos $\mathcal{E}_{\tau}\left[\Omega ; \mathbb{R}^{m}\right]$ e $\mathcal{N}_{\tau}\left[\Omega ; \mathbb{R}^{m}\right]$ em vez de $\left(\mathcal{E}_{\tau}[\Omega ; \mathbb{R}]\right)^{m}$ e $\left(\mathcal{N}_{\tau}[\Omega ; \mathbb{R}]\right)^{m}$, respectivamente.

Note que a aplicação

$$
\begin{aligned}
\left(\mathcal{G}_{\tau}(\Omega ; \mathbb{R})\right)^{m} & \longrightarrow \mathcal{G}_{\tau}\left(\Omega ; \mathbb{R}^{m}\right) \\
\left(u_{1_{\varepsilon}}+\mathcal{N}_{\tau}[\Omega ; \mathbb{R}], \cdots, u_{m_{\varepsilon}}+\mathcal{N}_{\tau}[\Omega ; \mathbb{R}]\right) & \longmapsto\left(u_{1_{\varepsilon}}, \cdots, u_{m_{\varepsilon}}\right)+\mathcal{N}_{\tau}\left[\Omega ; \mathbb{R}^{m}\right]
\end{aligned}
$$

é um isomorfismo de álgebras. Assim, para cada $m \in \mathbb{N}^{*}$, podemos identificar $\mathcal{G}_{\tau}\left(\Omega ; \mathbb{R}^{m}\right)$ $\operatorname{com}\left(\mathcal{G}_{\tau}(\Omega ; \mathbb{R})\right)^{m}$. Além disso, observe o seguinte.

Observação 1.3.4 Sejam $\Omega$ um aberto de $\mathbb{R}^{n}$ e $\left(u_{\varepsilon}\right)_{\varepsilon} \in(\mathcal{E}[\Omega ; \mathbb{R}])^{m}$. Tem-se

1. $\left(u_{\varepsilon}\right)_{\varepsilon} \in \mathcal{E}_{\tau}\left[\Omega ; \mathbb{R}^{m}\right]$ se, e somente se, para todo $\alpha \in \mathbb{N}^{n}$, existem $N \in \mathbb{N}, c>0$ e $\eta \in \mathbf{I}$ tais que

$$
\left|\partial^{\alpha} u_{\varepsilon}(x)\right| \leq c(1+|x|)^{N} \varepsilon^{-N}, \quad \forall(\varepsilon, x) \in \mathbf{I}_{\eta} \times \Omega
$$


2. $\left(u_{\varepsilon}\right)_{\varepsilon} \in \mathcal{N}_{\tau}\left[\Omega ; \mathbb{R}^{m}\right]$ se, e somente se, para todo $\alpha \in \mathbb{N}^{n}$, existe $N \in \mathbb{N}$ tal que, para todo $r \in \mathbb{N}$, existem $c>0$ e $\eta \in \mathbf{I}$ tais que

$$
\left|\partial^{\alpha} u_{\varepsilon}(x)\right| \leq c(1+|x|)^{N} \varepsilon^{r}, \quad \forall(\varepsilon, x) \in \mathbf{I}_{\eta} \times \Omega .
$$

Convém notar que, se $\Omega$ é um aberto de $\mathbb{R}^{n}$, então, todo $\left(u_{\varepsilon}\right)_{\varepsilon} \in \mathcal{E}_{\tau}\left[\Omega ; \mathbb{R}^{m}\right]$ está associado a um único elemento $u \in \mathcal{G}_{\tau}\left(\Omega ; \mathbb{R}^{m}\right)$ da seguinte forma

$$
u:=\left[\left(u_{\varepsilon}\right)_{\varepsilon}\right]=\left(u_{\varepsilon}\right)_{\varepsilon}+\mathcal{N}_{\tau}\left[\Omega ; \mathbb{R}^{m}\right]
$$

Nesse caso, dizemos que $\left(u_{\varepsilon}\right)_{\varepsilon} \in \mathcal{E}_{\tau}\left[\Omega ; \mathbb{R}^{m}\right]$ é um representante de $u \in \mathcal{G}_{\tau}\left(\Omega ; \mathbb{R}^{m}\right)$.

Em [GKOS] os autores apresentam um teorema de nulidade para funções moderadas temperadas em abertos "box" de $\mathbb{R}^{n}$, isto é, abertos da forma $J_{1} \times J_{2} \times \cdots \times J_{n}$, onde $J_{k}$ é um intervalo aberto de $\mathbb{R}$, para todo $1 \leq k \leq n$. Estudando a prova desse teorema percebemos que o mesmo ainda é verdadeiro para uma classe maior de abertos. O conjunto desses abertos será aqui denotado por $\mathcal{A}^{*}\left(\mathbb{R}^{n}\right)$ e é o seguinte:

Definição 1.3.5 Denotaremos por $\mathcal{A}^{*}\left(\mathbb{R}^{n}\right)$ o conjunto de todos os abertos $\Omega$ de $\mathbb{R}^{n}$ com a seguinte propriedade:

existe $\nu>0$ tal que, para quaisquer $x \in \Omega$ e $1 \leq i \leq n$, o segmento de extremidades $x$ e $x+\nu e_{i}$, ou o segmento de extremidades $x$ e $x-\nu e_{i}$, está contido em $\Omega$.

Exemplo 1.3.6 1. Todo aberto "box" de $\mathbb{R}^{n}$ está em $\mathcal{A}^{*}\left(\mathbb{R}^{n}\right)$.

2. O interior de um octógono regular com quatro dos seus lados paralelos aos eixos coordenados pertence a $\mathcal{A}^{*}\left(\mathbb{R}^{2}\right)$.

3. $\left.\bigcup_{n=2}^{\infty}\right] n-\frac{1}{n^{n}}, n+\frac{1}{n^{n}}\left[\notin \mathcal{A}^{*}(\mathbb{R})\right.$. 
Teorema 1.3.7 (Teorema de Nulidade em $\mathcal{E}_{\tau}\left[\Omega ; \mathbb{R}^{m}\right]$ ) Sejam $\Omega$ um aberto de $\mathbb{R}^{n}$ tal que $\Omega \in \mathcal{A}^{*}\left(\mathbb{R}^{n}\right)$ e $\left(u_{\varepsilon}\right)_{\varepsilon} \in \mathcal{E}_{\tau}\left[\Omega ; \mathbb{R}^{m}\right]$. São equivalentes as seguintes afirmações:

(a) $\left(u_{\varepsilon}\right)_{\varepsilon} \in \mathcal{N}_{\tau}\left[\Omega ; \mathbb{R}^{m}\right]$

(b) $\exists N \in \mathbb{N}, \forall r \in \mathbb{N}, \exists c>0 e \exists \eta \in \mathbf{I}$ tais que

$$
\left|u_{\varepsilon}(x)\right| \leq c(1+|x|)^{N} \varepsilon^{r}, \quad \forall(\varepsilon, x) \in \mathbf{I}_{\eta} \times \Omega
$$

Demonstração: Segue da definição de $\mathcal{N}_{\tau}\left[\Omega ; \mathbb{R}^{m}\right]$ que $(a)$ implica $(b)$. Para provarmos que (b) implica (a) observemos que basta termos a implicação para $m=1$.

Suponha $m=1$.

Provaremos, por indução sobre $|\alpha|$, onde $\alpha \in \mathbb{N}^{n}$, que

$\exists N \in \mathbb{N}, \forall r \in \mathbb{N}, \exists c>0$ e $\exists \eta \in \mathbf{I}$ tais que $\left|\partial^{\alpha} u_{\varepsilon}(x)\right| \leq c(1+|x|)^{N} \varepsilon^{r}, \quad \forall(\varepsilon, x) \in \mathbf{I}_{\eta} \times \Omega$.

É claro, por (b), que (1.7) é verdadeira para $|\alpha|=0$.

Seja $\alpha=\left(\alpha_{1}, \cdots, \alpha_{n}\right) \in \mathbb{N}^{n}$ com $|\alpha|>0$ e suponha (1.7) verdadeira para todo $\gamma \in \mathbb{N}^{n}$ $\operatorname{com}|\gamma|=|\alpha|-1$. Como $|\alpha|>0$, existe $1 \leq j \leq n$ tal que $\alpha_{j} \neq 0$, e assim

$$
\partial^{\alpha} u_{\varepsilon}=\frac{\partial}{\partial x_{j}}\left(\frac{\partial^{|\alpha|-1} u_{\varepsilon}}{\partial x_{1}^{\alpha_{1}} \cdots \partial x_{j}^{\alpha_{j}-1} \cdots \partial x_{n}^{\alpha_{n}}}\right)=\frac{\partial v_{\varepsilon}}{\partial x_{j}}
$$

onde $\left(v_{\varepsilon}\right)_{\varepsilon}:=\left(\frac{\partial^{|\alpha|-1} u_{\varepsilon}}{\partial x_{1}^{\alpha_{1}} \ldots \partial x_{j}^{\alpha_{j}-1} \cdots \partial x_{n}^{\alpha_{n}}}\right) \in \mathcal{E}_{\tau}[\Omega ; \mathbb{R}]$, para todo $\varepsilon \in \mathbf{I}$.

Para facilitar a escrita denote $\frac{\partial}{\partial x_{i}}$ por $\partial_{i}$ e $\frac{\partial^{2}}{\partial x_{i}^{2}}$ por $\partial_{i}^{2}$, onde $x_{i}$ é a i-ésima coordenada de um ponto arbitrário de $\mathbb{R}^{n}$ e $1 \leq i \leq n$.

Como $\left(v_{\varepsilon}\right)_{\varepsilon} \in \mathcal{E}_{\tau}[\Omega ; \mathbb{R}]$, existem $N_{1} \in \mathbb{N}, c_{1}>0$ e $\eta_{1} \in \mathbf{I}$ tais que

$$
\left|\partial_{i}^{2} v_{\varepsilon}(x)\right| \leq c_{1}(1+|x|)^{N_{1}} \varepsilon^{-N_{1}}, \quad \forall(\varepsilon, x) \in \mathbf{I}_{\eta_{1}} \times \Omega
$$

Da hipótese de indução, existe $N_{2} \in \mathbb{N}$ tal que

$$
\forall s \in \mathbb{N}, \exists c>0 \text { e } \exists \eta \in \mathbf{I} \text { tais que }\left|v_{\varepsilon}(x)\right| \leq c(1+|x|)^{N_{2}} \varepsilon^{s}, \quad \forall(\varepsilon, x) \in \mathbf{I}_{\eta} \times \Omega \text {. }
$$


Sejam $N:=\max \left\{N_{1}, N_{2}\right\}+1$ e $r \in \mathbb{N}$. Considere $s:=2 r+N$. Então por (1.9), existem $c_{2}>c_{1}$ e $\eta_{2} \in \mathbf{I} \operatorname{com} \eta_{2}<\eta_{1}$ tais que

$$
\left|v_{\varepsilon}(x)\right| \leq c_{2}(1+|x|)^{N_{2}} \varepsilon^{2 r+N}, \quad \forall(\varepsilon, x) \in \mathbf{I}_{\eta_{2}} \times \Omega
$$

e assim

$$
\left|v_{\varepsilon}(x)\right| \leq c_{2}(1+|x|)^{N} \varepsilon^{s}, \quad \forall(\varepsilon, x) \in \mathbf{I}_{\eta_{2}} \times \Omega
$$

Como $\Omega \in \mathcal{A}^{*}\left(\mathbb{R}^{n}\right)$, existe $\nu>0$ como na Definição 1.3.5. Considere $\eta:=\min \left\{\eta_{2}, \nu\right\}$ e note que, para todo $(\varepsilon, x) \in \mathbf{I}_{\eta} \times \Omega$, o segmento de extremidades $x$ e $x+\varepsilon^{r+N} e_{i}$ ou o segmento de extremidades $x$ e $x-\varepsilon^{r+N} e_{i}$ está contido em $\Omega$, onde $e_{i}$ é o i-ésimo elemento da base canônica de $\mathbb{R}^{n}$, pois os elementos desse segmento são da forma $x+t \varepsilon^{r+N} e_{i}$ ou $x-t \varepsilon^{r+N} e_{i}$ para $t \in[0,1]$. Dessa forma, usando o Polinômio de Taylor de ordem 1 , temos que, para $(\varepsilon, x) \in \mathbf{I}_{\eta} \times \Omega$, existe $y=x+\mu \varepsilon^{r+N} \in \Omega$, para algum $0 \leq|\mu| \leq 1$ tal que

$$
v_{\varepsilon}\left(x \pm \varepsilon^{r+N} e_{i}\right)=v_{\varepsilon}(x)+\partial_{i} v_{\varepsilon}(x) \varepsilon^{r+N}+\frac{1}{2} \partial_{i}^{2} v_{\varepsilon}(y)\left(\varepsilon^{r+N}\right)^{2}
$$

ou seja,

$$
\partial_{i} v_{\varepsilon}(x) \varepsilon^{r+N}=v_{\varepsilon}\left(x \pm \varepsilon^{r+N} e_{i}\right)-v_{\varepsilon}(x)-\frac{1}{2} \partial_{i}^{2} v_{\varepsilon}(y)\left(\varepsilon^{r+N}\right)^{2} .
$$

Assim, de (1.8) e (1.10), temos, para $(\varepsilon, x) \in \mathbf{I}_{\eta} \times \Omega$, que

$$
\begin{aligned}
\left|\partial_{i} v_{\varepsilon}(x)\right| \leq & \left(\left|v_{\varepsilon}\left(x \pm \varepsilon^{r+N} e_{i}\right)\right|+\left|v_{\varepsilon}(x)\right|\right) \varepsilon^{-r-N}+\frac{1}{2}\left|\partial_{i}^{2} v_{\varepsilon}(y)\right| \varepsilon^{r+N} \\
\leq & \left(c_{2}\left(1+\left|x \pm \varepsilon^{r+N} e_{i}\right|\right)^{N} \varepsilon^{2 r+N}+c_{2}(1+|x|)^{N} \varepsilon^{2 r+N}\right) \varepsilon^{-r-N}+ \\
& \quad+\frac{1}{2} c_{1}(1+|y|)^{N_{1}} \varepsilon^{-N_{1}} \varepsilon^{r+N} \\
\leq & c_{2}\left(1+|x|+\varepsilon^{r+N}\right)^{N} \varepsilon^{r}+c_{2}(1+|x|)^{N} \varepsilon^{r}+\frac{1}{2} c_{2}\left(1+|x|+|\mu| \varepsilon^{r+N}\right)^{N_{1}} \varepsilon^{r} \\
\leq & \frac{3}{2} c_{2}\left(1+|x|+\varepsilon^{r+N}\right)^{N} \varepsilon^{r}+c_{2}(1+|x|)^{N} \varepsilon^{r} \\
\leq & \frac{3}{2} c_{2}(2+|x|)^{N} \varepsilon^{r}+c_{2}(1+|x|)^{N} \varepsilon^{r} \\
\leq & \frac{3}{2} c_{2}(2+2|x|)^{N} \varepsilon^{r}+c_{2}(1+|x|)^{N} \varepsilon^{r} \\
\leq & 3.2^{N-1} c_{2}(1+|x|)^{N} \varepsilon^{r}+c_{2}(1+|x|)^{N} \varepsilon^{r} \\
\leq & \left(3.2^{N-1}+1\right) c_{2}(1+|x|)^{N} \varepsilon^{r} .
\end{aligned}
$$


Logo, se $c:=\left(3.2^{N-1}+1\right) c_{2}$, temos que

$$
\left|\partial_{i} v_{\varepsilon}(x)\right| \leq c(1+|x|)^{N} \varepsilon^{r}, \quad \forall(\varepsilon, x) \in \mathbf{I}_{\eta} \times \Omega
$$

e $\operatorname{assim}(1.7)$ é verdadeira.

No Teorema 1.3.7 é importante, como veremos no próximo exemplo, a hipótese de $\Omega$ ser um elemento de $\mathcal{A}^{*}\left(\mathbb{R}^{n}\right)$.

Exemplo 1.3.8 Sejam $\Omega:=\bigcup_{n=2}^{\infty} J_{n}$, onde $\left.J_{n}:=\right] n-\frac{1}{n^{n}}, n+\frac{1}{n^{n}}\left[e\left(u_{\varepsilon}\right)_{\varepsilon} \in \mathcal{E}[\Omega ; \mathbb{R}]\right.$ definida por

$$
u_{\varepsilon}(x)=\left\{\begin{array}{rl}
x-\left\lceil\varepsilon^{-1}\right\rceil & \text { se } x \in J_{\left\lceil\varepsilon^{-1}\right\rceil} \\
0 & \text { se } x \in \Omega \backslash J_{\left\lceil\varepsilon^{-1}\right\rceil}
\end{array},\right.
$$

onde $\left\lceil\varepsilon^{-1}\right\rceil$ denota o menor inteiro maior ou igual a $\varepsilon^{-1}$. Então $\left(u_{\varepsilon}\right)_{\varepsilon} \in \mathcal{E}_{\tau}[\Omega ; \mathbb{R}],\left(u_{\varepsilon}\right)_{\varepsilon}$ satisfaz a afirmação $(b)$ do Teorema 1.3 .7 e $\left(u_{\varepsilon}\right)_{\varepsilon} \notin \mathcal{N}_{\tau}[\Omega ; \mathbb{R}]$. (Note que $\Omega \notin \mathcal{A}^{*}(\mathbb{R})$ (Exemplo 1.3.6 (3.))).

De fato, considere $a_{\varepsilon}:=\left\lceil\varepsilon^{-1}\right\rceil$, para $\varepsilon \in \mathbf{I}$, e note que

$$
\begin{gathered}
\left|u_{\varepsilon}(x)\right| \leq\left(\frac{1}{a_{\varepsilon}}\right)^{a_{\varepsilon}} \leq \varepsilon^{a_{\varepsilon}} \leq \varepsilon^{1 / \varepsilon}, \quad \forall x \in J_{a_{\varepsilon}} ; \\
\left|\frac{d u_{\varepsilon}}{d x}(x)\right|=1 \quad \text { e }\left|\frac{d^{(p)} u_{\varepsilon}}{d x^{(p)}}(x)\right|=0, \quad \forall x \in J_{a_{\varepsilon}} \text { e } \forall p \in \mathbb{N} \text { com } p \geq 2 .
\end{gathered}
$$

De (1.11) e (1.12) temos que

$$
\left|\frac{d^{(s)} u_{\varepsilon}}{d x^{(s)}}(x)\right| \leq 1 \leq(1+|x|) \varepsilon^{-1}, \quad \forall(\varepsilon, x) \in \mathbf{I} \times \Omega \text { e } \forall s \in \mathbb{N},
$$

e $\operatorname{assim}\left(u_{\varepsilon}\right)_{\varepsilon} \in \mathcal{E}_{\tau}[\Omega ; \mathbb{R}]$. Para verificar que $\left(u_{\varepsilon}\right)_{\varepsilon}$ satisfaz a afirmação $(b)$ do Teorema 1.3.7, fixe $r \in \mathbb{N}$ e seja $\eta:=\frac{1}{r+1}$. Então, de (1.11), temos que

$$
\left|u_{\varepsilon}(x)\right| \leq \varepsilon^{1 / \varepsilon} \leq \varepsilon^{r} \leq(1+|x|) \varepsilon^{r}, \quad \forall(\varepsilon, x) \in \mathbf{I}_{\eta} \times \Omega .
$$


Finalmente, suponha, por absurdo, que $\left(u_{\varepsilon}\right)_{\varepsilon} \in \mathcal{N}_{\tau}[\Omega ; \mathbb{R}]$. Então existe $N \in \mathbb{N}$ tal que, para $r_{1}:=2 N$, existem $c>0$ e $\eta_{1} \in \mathbf{I} \operatorname{com} \eta_{1} \leq \frac{1}{2}$ tais que

$$
\left|\frac{d u_{\varepsilon}}{d x}(x)\right| \leq c(1+|x|)^{N} \varepsilon^{2 N}, \quad \forall(\varepsilon, x) \in \mathbf{I}_{\eta_{1}} \times \Omega,
$$

e assim, como $a_{\varepsilon} \in J_{\varepsilon}$, tem-se, para $\varepsilon \in \mathbf{I}_{\eta_{1}}$, que

$$
1=\frac{d u_{\varepsilon}}{d x}\left(a_{\varepsilon}\right) \leq c\left(1+\left|a_{\varepsilon}\right|\right)^{N} \varepsilon^{2 N} \leq c\left(1+\varepsilon^{-1}+1\right)^{N} \varepsilon^{2 N} \leq 2^{N} c \varepsilon^{-N} \varepsilon^{2 N} \leq 2^{N} c \varepsilon^{N},
$$

ou seja

$$
1 \leq 2^{N} c \varepsilon^{N}, \quad \forall \varepsilon \in \mathbf{I}_{\eta_{1}}
$$

o que é um absurdo.

O exemplo 1.3.8 é apresentado em [GKOS] como exemplo de um aberto não "box" no qual as afirmações $(a)$ e $(b)$ do Teorema 1.3.7 não são equivalentes. Contudo, vimos que, não é o fato do aberto não ser um "box" que garante $\left(u_{\varepsilon}\right)_{\varepsilon} \notin \mathcal{N}_{\tau}[\Omega ; \mathbb{R}]$, mas sim o fato dele não pertencer ao conjunto $\mathcal{A}^{*}(\mathbb{R})$.

De modo análogo ao caso $\mathcal{G}\left(\Omega ; \mathbb{R}^{m}\right)$, para definir a derivada de aplicações generalizadas temperadas observamos que, se $\Omega$ é um aberto de $\mathbb{R}^{n},\left(u_{\varepsilon}\right)_{\varepsilon} \in \mathcal{E}_{\tau}\left[\Omega ; \mathbb{R}^{m}\right], \alpha \in \mathbb{N}^{n}$ e $\left(\partial^{\alpha} u_{\varepsilon}\right)_{\varepsilon} \in \mathcal{E}\left[\Omega ; \mathbb{R}^{m}\right]$ é definida por

$$
\left(\partial^{\alpha} u_{\varepsilon}\right)_{\varepsilon}: x \in \Omega \longmapsto\left(\partial^{\alpha} u_{\varepsilon}\right)(x) \in \mathbb{R}^{m}
$$

então $\left(\partial^{\alpha} u_{\varepsilon}\right)_{\varepsilon} \in \mathcal{E}_{\tau}\left[\Omega ; \mathbb{R}^{m}\right]$. Assim, das propriedades lineares das derivadas, decorre que a aplicação

$$
\begin{aligned}
\partial^{\alpha}: \mathcal{E}_{\tau}\left[\Omega ; \mathbb{R}^{m}\right] & \longrightarrow \mathcal{E}_{\tau}\left[\Omega ; \mathbb{R}^{m}\right] \\
\left(u_{\varepsilon}\right)_{\varepsilon} & \longmapsto\left(\partial^{\alpha} u_{\varepsilon}\right)_{\varepsilon}
\end{aligned}
$$

é um homomorfismo de $\mathbb{R}$-espaços vetoriais. Observe ainda que $\partial^{\alpha}\left(\mathcal{E}_{\tau}\left[\Omega ; \mathbb{R}^{m}\right]\right) \subset \mathcal{E}_{\tau}\left[\Omega ; \mathbb{R}^{m}\right]$ e $\partial^{\alpha}\left(\mathcal{N}_{\tau}\left[\Omega ; \mathbb{R}^{m}\right]\right) \subset \mathcal{N}_{\tau}\left[\Omega ; \mathbb{R}^{m}\right]$. Portanto, do Teorema do Homomorfismo, existe uma única 
aplicação $\mathbb{R}$-linear $\partial^{\alpha}: \mathcal{G}_{\tau}\left(\Omega ; \mathbb{R}^{m}\right) \longrightarrow \mathcal{G}_{\tau}\left(\Omega ; \mathbb{R}^{m}\right)$ tal que o diagrama

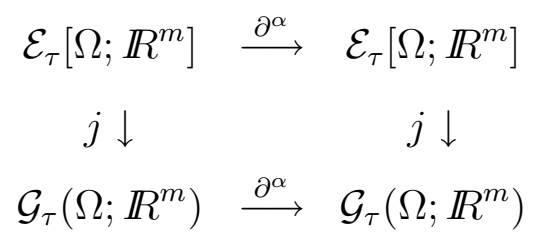

comuta, onde $j:\left(u_{\varepsilon}\right)_{\varepsilon} \in \mathcal{E}_{\tau}\left[\Omega ; \mathbb{R}^{m}\right] \longmapsto\left(u_{\varepsilon}\right)_{\varepsilon}+\mathcal{N}_{\tau}\left[\Omega ; \mathbb{R}^{m}\right]$. A partir desse fato, temos a seguinte definição.

Definição 1.3.9 Sejam $\Omega$ um aberto de $\mathbb{R}^{n}, u:=\left[\left(u_{\varepsilon}\right)_{\varepsilon}\right] \in \mathcal{G}_{\tau}\left(\Omega ; \mathbb{R}^{m}\right)$ e $\alpha \in \mathbb{N}^{n}$. Chamamos de derivada parcial de ordem $\alpha$ de $u$, o elemento $\partial^{\alpha} u:=\left[\left(\partial^{\alpha} u_{\varepsilon}\right)_{\varepsilon}\right] \in \mathcal{G}_{\tau}\left(\Omega ; \mathbb{R}^{m}\right)$.

A Definição 1.3.9 é compatível com a fórmula de Leibniz, ou seja,

$$
\begin{aligned}
\text { se } u, v \in \mathcal{G}_{\tau}(\Omega ; \mathbb{R}) \text { e } \alpha & \in \mathbb{N}^{n} \text {, então tem-se } \\
\partial^{\alpha}(u v) & =\sum_{0 \leq \beta \leq \alpha}\left(\begin{array}{l}
\alpha \\
\beta
\end{array}\right)\left(\partial^{\beta} u\right)\left(\partial^{\alpha-\beta} v\right) .
\end{aligned}
$$

A seguir definiremos a composta de aplicações generalizadas temperadas. Para isto utilizaremos o seguinte resultado.

Proposição 1.3.10 Sejam $\Omega$ um aberto de $\mathbb{R}^{n}, \Omega^{\prime}$ um aberto convexo de $\mathbb{R}^{p}$, $\left(v_{\varepsilon}\right)_{\varepsilon} \in \mathcal{E}_{\tau}\left[\Omega^{\prime} ; \mathbb{R}^{m}\right] \quad$ e $\left(u_{\varepsilon}\right)_{\varepsilon} \in \mathcal{E}_{\tau}\left[\Omega ; \mathbb{R}^{p}\right] \quad$ tal que $u_{\varepsilon}(\Omega) \subset \Omega^{\prime}, \quad \forall \varepsilon \in \mathbf{I} . \quad$ São verdadeiras as seguintes afirmações:

1. $\left(v_{\varepsilon} \circ u_{\varepsilon}\right)_{\varepsilon} \in \mathcal{E}_{\tau}\left[\Omega ; \mathbb{R}^{m}\right]$;

2. se $\left(w_{\varepsilon}\right)_{\varepsilon} \in \mathcal{E}_{\tau}\left[\Omega ; \mathbb{R}^{p}\right]$ é tal que $\left(w_{\varepsilon}-u_{\varepsilon}\right)_{\varepsilon} \in \mathcal{N}_{\tau}\left[\Omega ; \mathbb{R}^{p}\right]$ e $w_{\varepsilon}(\Omega) \subset \Omega^{\prime}$, para todo $\varepsilon \in \mathbf{I}$, então

$$
\left(v_{\varepsilon} \circ u_{\varepsilon}-v_{\varepsilon} \circ w_{\varepsilon}\right)_{\varepsilon} \in \mathcal{N}_{\tau}\left[\Omega ; \mathbb{R}^{m}\right] ;
$$


3. se $\left(f_{\varepsilon}\right)_{\varepsilon} \in \mathcal{E}_{\tau}\left[\Omega^{\prime} ; \mathbb{R}^{m}\right]$ é tal que $\left(f_{\varepsilon}-v_{\varepsilon}\right)_{\varepsilon} \in \mathcal{N}_{\tau}\left[\Omega^{\prime} ; \mathbb{R}^{m}\right]$, então

$$
\left(v_{\varepsilon} \circ u_{\varepsilon}-f_{\varepsilon} \circ u_{\varepsilon}\right)_{\varepsilon} \in \mathcal{N}_{\tau}\left[\Omega ; \mathbb{R}^{m}\right]
$$

4. se $\left(w_{\varepsilon}\right)_{\varepsilon}$ é como em 2. e $\left(f_{\varepsilon}\right)_{\varepsilon}$ é como em 3., então $\left(v_{\varepsilon} \circ u_{\varepsilon}-f_{\varepsilon} \circ w_{\varepsilon}\right)_{\varepsilon} \in \mathcal{N}_{\tau}\left[\Omega ; \mathbb{R}^{m}\right]$.

Demonstração: Considere $\left(v_{\varepsilon}\right)_{\varepsilon}:=\left(\left(v_{1_{\varepsilon}}, \cdots, v_{m_{\varepsilon}}\right)\right)_{\varepsilon}$ e $\left(f_{\varepsilon}\right)_{\varepsilon}:=\left(\left(f_{1_{\varepsilon}}, \cdots, f_{m_{\varepsilon}}\right)\right)_{\varepsilon}$. Então

$$
\begin{aligned}
& \left(v_{\varepsilon} \circ u_{\varepsilon}\right)_{\varepsilon}:=\left(\left(v_{1_{\varepsilon}} \circ u_{\varepsilon}, \cdots, v_{m_{\varepsilon}} \circ u_{\varepsilon}\right)\right)_{\varepsilon} \\
& \left(\left(v_{\varepsilon} \circ u_{\varepsilon}-v_{\varepsilon} \circ w_{\varepsilon}\right)_{\varepsilon}:=\left(v_{1_{\varepsilon}} \circ u_{\varepsilon}-v_{1_{\varepsilon}} \circ w_{\varepsilon}, \cdots, v_{m_{\varepsilon}} \circ u_{\varepsilon}-v_{m_{\varepsilon}} \circ w_{\varepsilon}\right)\right)_{\varepsilon} ; \\
& \left(\left(v_{\varepsilon} \circ u_{\varepsilon}-f_{\varepsilon} \circ u_{\varepsilon}\right)_{\varepsilon}:=\left(v_{1_{\varepsilon}} \circ u_{\varepsilon}-f_{1_{\varepsilon}} \circ u_{\varepsilon}, \cdots, v_{m_{\varepsilon}} \circ u_{\varepsilon}-f_{m_{\varepsilon}} \circ u_{\varepsilon}\right)\right)_{\varepsilon} .
\end{aligned}
$$

Portanto, se provarmos que o resultado é verdadeiro para $m=1$ teremos o resultado para o caso geral.

Suponhamos $m=1$.

Seja $\alpha \in \mathbb{N}^{n}$.

Para a prova de 1 . note que, $\partial^{\alpha}\left(v_{\varepsilon} \circ u_{\varepsilon}\right)$ é um polinômio em $\left(\partial^{\beta} v_{\varepsilon}\right) \circ u_{\varepsilon}$ e $\partial^{\gamma} u_{\varepsilon}$ com $|\beta|,|\gamma| \leq|\alpha|$. Portanto para obter 1 . basta provar que, para $\beta \in \mathbb{N}^{p}$ e $\gamma \in \mathbb{N}^{n}$ com $|\beta|,|\gamma| \leq|\alpha|$ existem $N \in \mathbb{N}, c>0$ e $\eta \in \mathbf{I}$ tais que

$$
\begin{gathered}
\left|\partial^{\gamma} u_{\varepsilon}(x)\right| \leq c(1+|x|)^{N} \varepsilon^{-N}, \quad \forall(\varepsilon, x) \in \mathbf{I}_{\eta} \times \Omega ; \\
\left|\left(\partial^{\beta} v_{\varepsilon}\right) \circ u_{\varepsilon}(x)\right| \leq c(1+|x|)^{N} \varepsilon^{-N}, \quad \forall(\varepsilon, x) \in \mathbf{I}_{\eta} \times \Omega .
\end{gathered}
$$

Como $\left(u_{\varepsilon}\right)_{\varepsilon} \in \mathcal{E}_{\tau}\left[\Omega ; \mathbb{R}^{p}\right]$ e $\left(v_{\varepsilon}\right)_{\varepsilon} \in \mathcal{E}_{\tau}\left[\Omega^{\prime} ; \mathbb{R}\right]$, existem $N_{1} \in \mathbb{N}, c_{1}>1$ e $\eta \in \mathbf{I}$ tais que

$\left|\partial^{\gamma} u_{\varepsilon}(x)\right| \leq c_{1}(1+|x|)^{N_{1}} \varepsilon^{-N_{1}}, \quad \forall(\varepsilon, x) \in \mathbf{I}_{\eta} \times \Omega, \quad \forall \gamma \in \mathbb{N}^{n} \operatorname{com}|\gamma| \leq|\alpha| ;$

$\left|\partial^{\beta} v_{\varepsilon}(y)\right| \leq c_{1}(1+|y|)^{N_{1}} \varepsilon^{-N_{1}}, \quad \forall(\varepsilon, y) \in \mathbf{I}_{\eta} \times \Omega^{\prime}, \quad \forall \beta \in \mathbb{N}^{p} \operatorname{com}|\beta| \leq|\alpha|$

Fixe $\beta \in \mathbb{N}^{p}$ e $\gamma \in \mathbb{N}^{n} \operatorname{com}|\beta|,|\gamma| \leq|\alpha|$. 
De $(1.15)$ e (1.16) temos, para $(\varepsilon, x) \in \mathbf{I}_{\eta} \times \Omega$, que

$$
\begin{aligned}
\left|\partial^{\beta} v_{\varepsilon}\left(u_{\varepsilon}(x)\right)\right| & \leq c_{1}\left(1+\left|u_{\varepsilon}(x)\right|\right)^{N_{1}} \varepsilon^{-N_{1}} \\
& \leq c_{1}\left(1+c_{1}(1+|x|)^{N_{1}} \varepsilon^{-N_{1}}\right)^{N_{1}} \varepsilon^{-N_{1}} \\
& \leq c_{1}\left((1+|x|)^{N_{1}} \varepsilon^{-N_{1}}+c_{1}(1+|x|)^{N_{1}} \varepsilon^{-N_{1}}\right)^{N_{1}} \varepsilon^{-N_{1}} \\
& \leq c_{1}\left(\left(1+c_{1}\right)(1+|x|)^{N_{1}} \varepsilon^{-N_{1}}\right)^{N_{1}} \varepsilon^{-N_{1}} \\
& \leq c_{1}\left(1+c_{1}\right)^{N_{1}}(1+|x|)^{N_{1}^{2}} \varepsilon^{-N_{1}^{2}-N_{1}} \\
& \leq c_{1}\left(1+c_{1}\right)^{N_{1}}(1+|x|)^{N_{1}^{2}+N_{1}} \varepsilon^{-N_{1}^{2}-N_{1}}
\end{aligned}
$$

Seja $c:=c_{1}\left(1+c_{1}\right)^{N_{1}}$ e $N:=N_{1}^{2}+N_{1}$, então

$$
\begin{gathered}
\left|\partial^{\gamma} u_{\varepsilon}(x)\right| \leq c(1+|x|)^{N} \varepsilon^{-N}, \quad \forall(\varepsilon, x) \in \mathbf{I}_{\eta} \times \Omega ; \\
\left|\partial^{\beta} v_{\varepsilon}\left(u_{\varepsilon}(x)\right)\right| \leq c(1+|x|)^{N} \varepsilon^{-N}, \quad \forall(\varepsilon, x) \in \mathbf{I}_{\eta} \times \Omega,
\end{gathered}
$$

o que prova (1.13) e (1.14).

Para 2. provaremos que, existe $N \in \mathbb{N}$ tal que, para todo $r \in \mathbb{N}$, existem $c>0$ e $\eta \in \mathbf{I}$ satisfazendo

$$
\left|v_{\varepsilon}\left(u_{\varepsilon}(x)\right)-v_{\varepsilon}\left(w_{\varepsilon}(x)\right)\right| \leq c(1+|x|)^{N} \varepsilon^{r}, \quad \forall(\varepsilon, x) \in \mathbf{I}_{\eta} \times \Omega,
$$

e assim, do Teorema de Nulidade em $\mathcal{E}_{\tau}[\Omega ; \mathbb{R}]$ (Teorema 1.3.7), seguirá que

$$
\left(v_{\varepsilon} \circ u_{\varepsilon}-v_{\varepsilon} \circ w_{\varepsilon}\right)_{\varepsilon} \in \mathcal{N}_{\tau}[\Omega ; \mathbb{R}]
$$

$\operatorname{De}\left(v_{\varepsilon}\right)_{\varepsilon} \in \mathcal{E}_{\tau}\left[\Omega^{\prime} ; \mathbb{R}\right]$ e $\left(u_{\varepsilon}\right)_{\varepsilon},\left(w_{\varepsilon}\right)_{\varepsilon} \in \mathcal{E}_{\tau}\left[\Omega ; \mathbb{R}^{p}\right]$, existem $N_{2} \in \mathbb{N}, c_{2}>1$ e $\eta_{2} \in \mathbf{I}$ tais que

$$
\begin{gathered}
\left|\partial^{\alpha} v_{\varepsilon}(y)\right| \leq c_{2}(1+|y|)^{N_{2}} \varepsilon^{-N_{2}}, \quad \forall(\varepsilon, y) \in \mathbf{I}_{\eta_{2}} \times \Omega^{\prime}, \forall \alpha \in \mathbb{N}^{p} \operatorname{com}|\alpha|=1 ; \\
\left|u_{\varepsilon}(x)\right| \leq c_{2}(1+|x|)^{N_{2}} \varepsilon^{-N_{2}}, \quad \forall(\varepsilon, x) \in \mathbf{I}_{\eta_{2}} \times \Omega ; \\
\left|w_{\varepsilon}(x)\right| \leq c_{2}(1+|x|)^{N_{2}} \varepsilon^{-N_{2}}, \quad \forall(\varepsilon, x) \in \mathbf{I}_{\eta_{2}} \times \Omega .
\end{gathered}
$$


De $\left(n_{\varepsilon}\right)_{\varepsilon}:=\left(w_{\varepsilon}-u_{\varepsilon}\right)_{\varepsilon} \in \mathcal{N}_{\tau}\left[\Omega ; \mathbb{R}^{p}\right]$, existe $N_{3} \in \mathbb{N}$ com $N_{3}>N_{2}$ tal que, para todo $q \in \mathbb{N}$, existem $c_{q}>c_{2}$ e $\eta_{q} \in \mathbf{I}$ com $\eta_{q}<\eta_{2}$ satisfazendo

$$
\left|n_{\varepsilon}(x)\right| \leq c_{q}(1+|x|)^{N_{3}} \varepsilon^{q}, \quad \forall(\varepsilon, x) \in \mathbf{I}_{\eta_{q}} \times \Omega .
$$

Considere $N:=N_{2}^{2}+N_{3}$ e seja $r \in \mathbb{N}$. Então, para $q:=r+N$, existem $c_{3}:=c_{q}$ e $\eta_{3}:=\eta_{q}$ como em (1.20), isto é

$$
\left|n_{\varepsilon}(x)\right| \leq c_{3}(1+|x|)^{N_{3}} \varepsilon^{q}, \quad \forall(\varepsilon, x) \in \mathbf{I}_{\eta_{3}} \times \Omega .
$$

Fixemos $(\varepsilon, x) \in \mathbf{I}_{\eta_{3}} \times \Omega$. Usando o fato de $\Omega^{\prime}$ ser aberto convexo e o Teorema do Valor Médio, existe $\sigma \in] 0,1[$ tal que

$$
\begin{aligned}
\left|v_{\varepsilon}\left(u_{\varepsilon}(x)\right)-v_{\varepsilon}\left(w_{\varepsilon}(x)\right)\right| & =\left|\left\langle u_{\varepsilon}(x)-w_{\varepsilon}(x), \nabla v_{\varepsilon}\left(w_{\varepsilon}(x)+\sigma n_{\varepsilon}(x)\right)\right\rangle\right| \\
& \leq\left|n_{\varepsilon}(x)\right|\left|\nabla v_{\varepsilon}\left(w_{\varepsilon}(x)+\sigma n_{\varepsilon}(x)\right)\right|,
\end{aligned}
$$

onde $\langle$,$\rangle denota o produto interno usual de \mathbb{R}^{p}$.

De (1.17), (1.18), (1.19) e (1.21), temos que

$$
\begin{aligned}
\left|v_{\varepsilon}\left(u_{\varepsilon}(x)\right)-v_{\varepsilon}\left(w_{\varepsilon}(x)\right)\right| & \leq\left|n_{\varepsilon}(x)\right|\left|\nabla v_{\varepsilon}\left(w_{\varepsilon}(x)+\sigma n_{\varepsilon}(x)\right)\right| \\
& \leq c_{3}(1+|x|)^{N_{3}} \varepsilon^{q} p c_{2}\left(1+\left|w_{\varepsilon}(x)+\sigma n_{\varepsilon}(x)\right|\right)^{N_{2}} \varepsilon^{-N_{2}} \\
& \leq c_{3}(1+|x|)^{N_{3}} \varepsilon^{q} p c_{2}\left(1+\left|w_{\varepsilon}(x)\right|+\left|\sigma n_{\varepsilon}(x)\right|\right)^{N_{2}} \varepsilon^{-N_{2}} \\
& \leq c_{3}(1+|x|)^{N_{3}} \varepsilon^{q} p c_{2}\left(1+\left|w_{\varepsilon}(x)\right|+\left|n_{\varepsilon}(x)\right|\right)^{N_{2}} \varepsilon^{-N_{2}} \\
& \leq c_{3}(1+|x|)^{N_{3}} \varepsilon^{q} p c_{2}\left(1+\left|w_{\varepsilon}(x)\right|+\left|u_{\varepsilon}(x)\right|+\left|w_{\varepsilon}(x)\right|\right)^{N_{2}} \varepsilon^{-N_{2}} \\
& \leq c_{3}(1+|x|)^{N_{3}} \varepsilon^{q} p c_{2}\left(1+3 c_{2}(1+|x|)^{N_{2}} \varepsilon^{-N_{2}}\right)^{N_{2}} \varepsilon^{-N_{2}} \\
& \leq c_{3}(1+|x|)^{N_{3}} \varepsilon^{q} p c_{2}\left(1+3 c_{2}\right)^{N_{2}}(1+|x|)^{N_{2}^{2}} \varepsilon^{-N_{2}^{2}-N_{2}} \\
& \leq p c_{3}^{2}\left(1+3 c_{3}\right)^{N_{3}}(1+|x|)^{N_{2}{ }^{2}+N_{3}} \varepsilon^{q-N_{2}^{2}-N_{2}} .
\end{aligned}
$$

Seja $c:=p c_{3}^{2}\left(1+3 c_{3}\right)^{N_{3}}$. Então

$$
\left|v_{\varepsilon}\left(u_{\varepsilon}(x)\right)-v_{\varepsilon}\left(w_{\varepsilon}(x)\right)\right| \leq c(1+|x|)^{N} \varepsilon^{r}, \quad \forall(\varepsilon, x) \in \mathbf{I}_{\eta_{3}} \times \Omega .
$$


Para 3. provaremos que, existe $N \in \mathbb{N}$ tal que, para todo $s \in \mathbb{N}$, existem $c>0$ e $\eta \in \mathbf{I}$ tais que

$$
\left|v_{\varepsilon}\left(u_{\varepsilon}(x)\right)-f_{\varepsilon}\left(u_{\varepsilon}(x)\right)\right| \leq c(1+|x|)^{N} \varepsilon^{s}, \quad \forall(\varepsilon, x) \in \mathbf{I}_{\eta} \times \Omega,
$$

e, do Teorema de Nulidade em $\mathcal{E}_{\tau}[\Omega ; \mathbb{R}]$ (Teorema 1.3.7), seguirá que

$$
\left(v_{\varepsilon} \circ u_{\varepsilon}-f_{\varepsilon} \circ u_{\varepsilon}\right)_{\varepsilon} \in \mathcal{N}_{\tau}[\Omega ; \mathbb{R}]
$$

Sejam $N_{2}, c_{2}$ e $\eta_{2}$ como em (1.18).

De $\left(m_{\varepsilon}\right)_{\varepsilon}:=\left(v_{\varepsilon}-f_{\varepsilon}\right)_{\varepsilon} \in \mathcal{N}_{\tau}\left[\Omega^{\prime} ; \mathbb{R}\right]$, existe $N_{4} \in \mathbb{N}$ com $N_{4}>N_{2}$ tal que, para todo $k \in \mathbb{N}$, existem $c_{k}>c_{2}$ e $\eta_{k} \in \mathbf{I}$ com $\eta_{k}<\eta_{2}$ satisfazendo

$$
\left|m_{\varepsilon}(y)\right| \leq c_{k}(1+|y|)^{N_{4}} \varepsilon^{k}, \quad \forall(\varepsilon, y) \in \mathbf{I}_{\eta_{k}} \times \Omega^{\prime}
$$

Considere $N:=N_{4}^{2}$ e seja $s \in \mathbb{N}$. Então, para $k:=s+N$, existem $c_{4}:=c_{k}$ e $\eta_{4}:=\eta_{k}$ como em (1.22), isto é

$$
\left|m_{\varepsilon}(y)\right| \leq c_{4}(1+|y|)^{N_{4}} \varepsilon^{k}, \quad \forall(\varepsilon, y) \in \mathbf{I}_{\eta_{4}} \times \Omega^{\prime}
$$

De (1.18) e (1.23), temos que

$$
\begin{aligned}
\left|v_{\varepsilon}\left(u_{\varepsilon}(x)\right)-f_{\varepsilon}\left(u_{\varepsilon}(x)\right)\right| & =\left|m_{\varepsilon}\left(u_{\varepsilon}(x)\right)\right| \\
& \leq c_{4}\left(1+\left|u_{\varepsilon}(x)\right|\right)^{N^{4}} \varepsilon^{k} \\
& \leq c_{4}\left(1+c_{2}(1+|x|)^{N_{2}} \varepsilon^{-N_{2}}\right)^{N_{4}} \varepsilon^{k} \\
& \leq c_{4}\left((1+|x|)^{N_{2}} \varepsilon^{-N_{2}}+c_{2}(1+|x|)^{N_{2}} \varepsilon^{-N_{2}}\right)^{N_{4}} \varepsilon^{k} \\
& \leq c_{4}\left(\left(1+c_{2}\right)(1+|x|)^{N_{2}} \varepsilon^{-N_{2}}\right)^{N_{4}} \varepsilon^{k} \\
& \leq c_{4}\left(1+c_{2}\right)^{N_{4}}(1+|x|)^{N_{2} N_{4}} \varepsilon^{-N_{2} N_{4}} \varepsilon^{k} \\
& \leq c_{4}\left(1+c_{2}\right)^{N_{4}}(1+|x|)^{N_{4}^{2}} \varepsilon^{k-N_{4}^{2}} .
\end{aligned}
$$

Seja $c:=c_{4}\left(1+c_{2}\right)^{N_{4}}$. Então

$$
\left|v_{\varepsilon}\left(u_{\varepsilon}(x)\right)-f_{\varepsilon}\left(u_{\varepsilon}(x)\right)\right| \leq c(1+|x|)^{N} \varepsilon^{s}, \quad \forall(\varepsilon, x) \in \mathbf{I}_{\eta} \times \Omega .
$$


Finalmente para 4. basta observar que

$$
v_{\varepsilon} \circ u_{\varepsilon}-f_{\varepsilon} \circ w_{\varepsilon}=\left(v_{\varepsilon} \circ u_{\varepsilon}-v_{\varepsilon} \circ w_{\varepsilon}\right)+\left(v_{\varepsilon} \circ w_{\varepsilon}-f_{\varepsilon} \circ w_{\varepsilon}\right),
$$

usar 2. e observar que 3. é verdadeira se $\left(u_{\varepsilon}\right)_{\varepsilon}$ for substituído por $\left(w_{\varepsilon}\right)_{\varepsilon}$.

Se $\Omega$ e $\Omega^{\prime}$ são abertos de $\mathbb{R}^{n}$ e $\mathbb{R}^{p}$, respectivamente, definimos

$\mathcal{G}_{\tau}\left(\Omega ; \Omega^{\prime}\right):=\left\{u \in \mathcal{G}_{\tau}\left(\Omega ; \mathbb{R}^{p}\right) \mid \exists\left(u_{\varepsilon}\right)_{\varepsilon}\right.$ representante de u tal que $\left.u_{\varepsilon}(\Omega) \subset \Omega^{\prime}, \forall \varepsilon \in \mathbf{I}\right\}$.

A partir da Proposição 1.3.10 temos a seguinte definição.

Definição 1.3.11 Sejam $\Omega$ um aberto de $\mathbb{R}^{n}, \Omega^{\prime}$ um aberto convexo de $\mathbb{R}^{p}$, $u \in \mathcal{G}_{\tau}\left(\Omega ; \Omega^{\prime}\right)$ e $v \in \mathcal{G}_{\tau}\left(\Omega^{\prime} ; \mathbb{R}^{m}\right)$. Chamamos de composta de u e v, e denotamos por $v \circ u$, o elemento de $\mathcal{G}_{\tau}\left(\Omega ; \mathbb{R}^{m}\right)$ definido por

$$
v \circ u:=\left(v_{\varepsilon} \circ u_{\varepsilon}\right)_{\varepsilon}+\mathcal{N}_{\tau}\left[\Omega ; \mathbb{R}^{m}\right]
$$

onde $\left(v_{\varepsilon}\right)_{\varepsilon}$ é um representante qualquer de $v$ e $\left(u_{\varepsilon}\right)_{\varepsilon}$ é um representante qualquer de $u$ de modo que $u_{\varepsilon}(\Omega) \subset \Omega^{\prime}$, para todo $\varepsilon \in I$.

Nos próximos resultados iremos comparar os conjuntos $\mathcal{E}_{\tau}\left[\Omega ; \mathbb{R}^{m}\right]$ e $\mathcal{E}_{M}\left[\Omega ; \mathbb{R}^{m}\right]$, e as álgebras $\mathcal{G}_{\tau}\left(\Omega ; \mathbb{R}^{m}\right)$ e $\mathcal{G}\left(\Omega ; \mathbb{R}^{m}\right)$.

Proposição 1.3.12 Seja $\Omega$ um aberto de $\mathbb{R}^{n}$. Então

1. $\mathcal{E}_{\tau}\left[\Omega ; \mathbb{R}^{m}\right] \subset \mathcal{E}_{M}\left[\Omega ; \mathbb{R}^{m}\right]$

2. $\mathcal{N}_{\tau}\left[\Omega ; \mathbb{R}^{m}\right] \subset \mathcal{N}\left[\Omega ; \mathbb{R}^{m}\right]$. 
Demonstração: Basta observar que, se $K \subset \subset \Omega$ então, para todo $N \in \mathbb{N}$, a função $x \in \Omega \longmapsto(1+|x|)^{N}$ restrita a $K$ é limitada.

A partir da Proposição 1.3.12(1.) podemos definir a aplicação linear

$$
\begin{aligned}
\Psi: \mathcal{G}_{\tau}\left(\Omega ; \mathbb{R}^{m}\right) & \longrightarrow \mathcal{G}\left(\Omega ; \mathbb{R}^{m}\right) \\
\left(u_{\varepsilon}\right)_{\varepsilon}+\mathcal{N}_{\tau}\left[\Omega ; \mathbb{R}^{m}\right] & \longmapsto\left(u_{\varepsilon}\right)_{\varepsilon}+\mathcal{N}\left[\Omega ; \mathbb{R}^{m}\right]
\end{aligned}
$$

Convém observar que a aplicação $\Psi$, como mostra o exemplo a seguir (baseado no exemplo encontrado em [NPS]), pode não ser injetora.

Exemplo 1.3.13 Seja $\varphi \in \mathscr{C}^{\infty}(\mathbb{R} ; \mathbb{R})$ tal que

$$
\left.\varphi \equiv 1 \text { em }\left[\frac{-1}{4}, \frac{1}{4}\right] \text { e } \varphi \equiv 0 \text { em } \mathbb{R} \backslash\right] \frac{-1}{2}, \frac{1}{2}[
$$

Se $\left(v_{\varepsilon}\right)_{\varepsilon}$ é definida por

$$
v_{\varepsilon}(x)=\varphi\left(x-\varepsilon^{-1}\right), \quad \forall(\varepsilon, x) \in \mathbf{I} \times \mathbb{R},
$$

$\operatorname{então}\left(v_{\varepsilon}\right)_{\varepsilon} \in \mathcal{E}_{\tau}[\mathbb{R} ; \mathbb{R}],\left(v_{\varepsilon}\right)_{\varepsilon} \in \mathcal{N}[\mathbb{R} ; \mathbb{R}] \operatorname{mas}\left(v_{\varepsilon}\right)_{\varepsilon} \notin \mathcal{N}_{\tau}[\mathbb{R} ; \mathbb{R}]$.

De fato, de $\varphi$ ser limitada e $\frac{d^{p} v_{\varepsilon}}{d x^{p}}(x)=\frac{d^{p} \varphi}{d x^{p}}\left(x-\varepsilon^{-1}\right)$, para todo $(\varepsilon, p, x) \in \mathbf{I} \times \mathbb{N} \times \mathbb{R}$, é claro que $\left(v_{\varepsilon}\right)_{\varepsilon} \in \mathcal{E}_{\tau}[\mathbb{R} ; \mathbb{R}]$ e $\left(v_{\varepsilon}\right)_{\varepsilon} \in \mathcal{E}_{M}[\mathbb{R} ; \mathbb{R}]$. Para verificar que $\left(v_{\varepsilon}\right)_{\varepsilon} \in \mathcal{N}[\mathbb{R} ; \mathbb{R}]$, considere $p \in \mathbb{N}, K \subset \subset \mathbb{R}, T>0$ tal que $K \subset]-T, T\left[\right.$ e $\eta:=\frac{1}{T+1}$. Então

$$
\left.A:=\left\{x-\varepsilon^{-1} \mid(\varepsilon, x) \in \mathbf{I}_{\eta} \times\right]-T, T[\} \subset\right]-\infty,-1[,
$$

e assim, como $\left.\varphi\right|_{]_{-\infty,-1[}} \equiv 0$, temos que $\frac{d^{p} v_{\varepsilon}}{d x^{p}} \equiv 0$ em $]-T, T\left[\right.$. Logo $\frac{d^{p} v_{\varepsilon}}{d x^{p}} \equiv 0$ em $K$, ou $\operatorname{seja}\left(v_{\varepsilon}\right)_{\varepsilon} \in \mathcal{N}[\mathbb{R} ; \mathbb{R}]$. Para finalizar, suponha, por absurdo, que $\left(v_{\varepsilon}\right)_{\varepsilon} \in \mathcal{N}_{\tau}[\mathbb{R} ; \mathbb{R}]$. Então existe $N \in \mathbb{N}$ tal que, para $r:=N+1$, existem $c>1$ e $\eta_{1} \in \mathbf{I}$ tais que

$$
\left|v_{\varepsilon}(x)\right| \leq c(1+|x|)^{N} \varepsilon^{r}, \quad \forall(\varepsilon, x) \in \mathbf{I}_{\eta_{1}} \times \mathbb{R}
$$


isto é,

$$
\left|v_{\varepsilon}(x)\right| \leq c(1+|x|)^{N} \varepsilon^{N+1}, \quad \forall(\varepsilon, x) \in \mathbf{I}_{\eta_{1}} \times \mathbb{R} .
$$

Portanto,

$$
1=\varphi(0)=v_{\varepsilon}\left(\frac{1}{\varepsilon}\right) \leq c\left(1+\frac{1}{\varepsilon}\right)^{N} \varepsilon^{N+1} \leq c\left(\frac{1}{\varepsilon}+\frac{1}{\varepsilon}\right)^{N} \varepsilon^{N+1} \leq 2^{N} c \varepsilon, \quad \forall \varepsilon \in \mathbf{I}_{\eta_{1}},
$$

o que é um absurdo. $\operatorname{Logo}\left(v_{\varepsilon}\right)_{\varepsilon} \notin \mathcal{N}_{\tau}[\mathbb{R} ; \mathbb{R}]$.

Vimos que, se $\left(u_{\varepsilon}\right)_{\varepsilon} \in \mathcal{E}_{M}\left[\Omega ; \mathbb{R}^{p}\right]$ e $\left(v_{\varepsilon}\right)_{\varepsilon} \in \mathcal{E}_{M}\left[\mathbb{R}^{p} ; \mathbb{R}^{m}\right]$ a função $\left(v_{\varepsilon} \circ u_{\varepsilon}\right)_{\varepsilon}$ pode não pertencer a $\mathcal{E}_{M}\left[\Omega ; \mathbb{R}^{m}\right]$. Contudo, é fácil verificar que, se $\left(v_{\varepsilon}\right)_{\varepsilon} \in \mathcal{E}_{\tau}\left[\mathbb{R}^{p} ; \mathbb{R}^{m}\right]$, então $\left(v_{\varepsilon} \circ u_{\varepsilon}\right)_{\varepsilon} \in \mathcal{E}_{M}\left[\Omega ; \mathbb{R}^{m}\right]$. Portanto é natural compor elemento de $\mathcal{G}\left(\Omega ; \mathbb{R}^{p}\right)$ com elemento de $\mathcal{G}_{\tau}\left(\mathbb{R}^{p} ; \mathbb{R}^{m}\right)$ e obter um elemento de $\mathcal{G}\left(\Omega ; \mathbb{R}^{m}\right)$. É isso que faremos a seguir.

Proposição 1.3.14 Sejam $\Omega$ um aberto de $\mathbb{R}^{n}$, $\Omega^{\prime}$ um aberto convexo de $\mathbb{R}^{p}$, $\left(v_{\varepsilon}\right)_{\varepsilon} \in \mathcal{E}_{\tau}\left[\Omega^{\prime} ; \mathbb{R}^{m}\right] \quad$ e $\left(u_{\varepsilon}\right)_{\varepsilon} \in \mathcal{E}_{M}\left[\Omega ; \mathbb{R}^{p}\right]$ tal que $u_{\varepsilon}(\Omega) \subset \Omega^{\prime}, \forall \varepsilon \in \mathbf{I}$. São verdadeiras as seguintes afirmações:

1. $\left(v_{\varepsilon} \circ u_{\varepsilon}\right)_{\varepsilon} \in \mathcal{E}_{M}\left[\Omega ; \mathbb{R}^{m}\right]$;

2. se $\left(w_{\varepsilon}\right)_{\varepsilon} \in \mathcal{E}_{M}\left[\Omega ; \mathbb{R}^{p}\right]$ é tal que $\left(w_{\varepsilon}-u_{\varepsilon}\right)_{\varepsilon} \in \mathcal{N}\left[\Omega ; \mathbb{R}^{p}\right]$ e $w_{\varepsilon}(\Omega) \subset \Omega^{\prime}$, para todo $\varepsilon \in \mathbf{I}$, então

$$
\left(v_{\varepsilon} \circ u_{\varepsilon}-v_{\varepsilon} \circ w_{\varepsilon}\right)_{\varepsilon} \in \mathcal{N}\left[\Omega ; \mathbb{R}^{m}\right]
$$

3. se $\left(f_{\varepsilon}\right)_{\varepsilon} \in \mathcal{E}_{\tau}\left[\Omega^{\prime} ; \mathbb{R}^{m}\right]$ é tal que $\left(f_{\varepsilon}-v_{\varepsilon}\right)_{\varepsilon} \in \mathcal{N}_{\tau}\left[\Omega^{\prime} ; \mathbb{R}^{m}\right]$, então

$$
\left(v_{\varepsilon} \circ u_{\varepsilon}-f_{\varepsilon} \circ u_{\varepsilon}\right)_{\varepsilon} \in \mathcal{N}\left[\Omega ; \mathbb{R}^{m}\right]
$$

4. se $\left(w_{\varepsilon}\right)_{\varepsilon}$ é como em 2. e $\left(f_{\varepsilon}\right)_{\varepsilon}$ é como em 3., então

$$
\left(v_{\varepsilon} \circ u_{\varepsilon}-f_{\varepsilon} \circ w_{\varepsilon}\right)_{\varepsilon} \in \mathcal{N}\left[\Omega ; \mathbb{R}^{m}\right]
$$


Demonstração: Sejam $\alpha \in \mathbb{N}^{n}$ e $K \subset \subset \Omega$.

Para 1. proceda como na prova da Proposição 1.3.10 substituindo (1.13) por

$$
\left|\partial^{\gamma} u_{\varepsilon}(x)\right| \leq c \varepsilon^{-N}, \quad \forall(\varepsilon, x) \in \mathbf{I}_{\eta} \times K
$$

substituindo (1.14) por

$$
\left|\left(\partial^{\beta} v_{\varepsilon}\right) \circ u_{\varepsilon}(x)\right| \leq c \varepsilon^{-N}, \quad \forall(\varepsilon, x) \in \mathbf{I}_{\eta} \times K,
$$

substituindo $\left(u_{\varepsilon}\right)_{\varepsilon} \in \mathcal{E}_{\tau}\left[\Omega ; \mathbb{R}^{p}\right]$ por $\left(u_{\varepsilon}\right)_{\varepsilon} \in \mathcal{E}_{M}\left[\Omega ; \mathbb{R}^{p}\right]$, substituindo (1.15) por

$$
\left|\partial^{\gamma} u_{\varepsilon}(x)\right| \leq c_{1} \varepsilon^{-N_{1}}, \quad \forall(\varepsilon, x) \in \mathbf{I}_{\eta_{1}} \times K, \quad \forall \gamma \in \mathbb{N}^{n} \operatorname{com}|\gamma| \leq|\alpha|
$$

e mantendo (1.16).

Para 2. basta provarmos, pelo Teorema da Nulidade em $\mathcal{E}_{M}[\Omega ; \mathbb{R}]$ (Teorema 1.2.5) que, para todo $r \in \mathbb{N}$, existem $c>0$ e $\eta \in \mathbf{I}$ satisfazendo

$$
\left|v_{\varepsilon}\left(u_{\varepsilon}(x)\right)-v_{\varepsilon}\left(w_{\varepsilon}(x)\right)\right| \leq c \varepsilon^{r}, \quad \forall(\varepsilon, x) \in \mathbf{I}_{\eta} \times K .
$$

Para isso, dado $r \in \mathbb{N}$, basta procedermos como na prova da Proposição 1.3.10 substituindo $\left(u_{\varepsilon}\right)_{\varepsilon},\left(w_{\varepsilon}\right)_{\varepsilon} \in \mathcal{E}_{\tau}\left[\Omega ; \mathbb{R}^{p}\right]$ por $\left(u_{\varepsilon}\right)_{\varepsilon},\left(w_{\varepsilon}\right)_{\varepsilon} \in \mathcal{E}_{M}\left[\Omega ; \mathbb{R}^{p}\right]$, mantendo (1.17), substituindo (1.18) por

$$
\left|u_{\varepsilon}(x)\right| \leq c_{2} \varepsilon^{-N_{2}}, \quad \forall(\varepsilon, x) \in \mathbf{I}_{\eta_{2}} \times K,
$$

substituindo (1.19) por

$$
\left|w_{\varepsilon}(x)\right| \leq c_{2} \varepsilon^{-N_{2}}, \quad \forall(\varepsilon, x) \in \mathbf{I}_{\eta_{2}} \times K,
$$

substituindo $\left(n_{\varepsilon}\right)_{\varepsilon}:=\left(w_{\varepsilon}-u_{\varepsilon}\right)_{\varepsilon} \in \mathcal{N}_{\tau}\left[\Omega ; \mathbb{R}^{p}\right]$ por $\left(n_{\varepsilon}\right)_{\varepsilon}:=\left(w_{\varepsilon}-u_{\varepsilon}\right)_{\varepsilon} \in \mathcal{N}\left[\Omega ; \mathbb{R}^{p}\right]$, substituindo (1.20) por

$$
\forall q \in \mathbb{N}, \exists c_{q}>c_{2}, \exists \eta_{q} \in \mathbf{I} \text { com } \eta_{q}<\eta_{2} \text { tal que }\left|n_{\varepsilon}(x)\right| \leq c_{q} \varepsilon^{q}, \quad \forall(\varepsilon, x) \in \mathbf{I}_{\eta_{q}} \times K,
$$

substituindo $q:=r+N$ por $q:=r+N_{2}^{2}+N_{2}$, substituindo (1.21) por

$$
\left|n_{\varepsilon}(x)\right| \leq c_{3} \varepsilon^{q}=c_{3} \varepsilon^{r+N_{2}^{2}+N_{2}}, \quad \forall(\varepsilon, x) \in \mathbf{I}_{\eta_{3}} \times K .
$$


Para 3. e 4. proceda como na prova da Proposição 1.3.10 fazendo as mudanças realizadas anteriormente e substituindo o Teorema 1.3.7 pelo Teorema 1.2.5.

Se $\Omega$ e $\Omega^{\prime}$ são abertos de $\mathbb{R}^{n}$ e $\mathbb{R}^{p}$, respectivamente, definimos $\mathcal{G}\left(\Omega ; \Omega^{\prime}\right):=\left\{u \in \mathcal{G}\left(\Omega ; \mathbb{R}^{p}\right) \mid \exists\left(u_{\varepsilon}\right)_{\varepsilon}\right.$ representante de $u$ tal que $\left.u_{\varepsilon}(\Omega) \subset \Omega^{\prime}, \forall \varepsilon \in \mathbf{I}\right\}$.

A partir da Proposição 1.3.14 temos a seguinte definição.

Definição 1.3.15 Sejam $\Omega$ um aberto de $\mathbb{R}^{n}, \Omega^{\prime}$ um aberto convexo de $\mathbb{R}^{p}, u \in \mathcal{G}\left(\Omega ; \Omega^{\prime}\right)$ e $v \in \mathcal{G}_{\tau}\left(\Omega^{\prime} ; \mathbb{R}^{m}\right)$. A composta de u e v, denotada por $v \circ u$, é o elemento de $\mathcal{G}\left(\Omega ; \mathbb{R}^{m}\right)$ definido por

$$
v \circ u:=\left(v_{\varepsilon} \circ u_{\varepsilon}\right)_{\varepsilon}+\mathcal{N}\left[\Omega ; \mathbb{R}^{m}\right]
$$

onde $\left(v_{\varepsilon}\right)_{\varepsilon}$ é um representante qualquer de $v$ e $\left(u_{\varepsilon}\right)_{\varepsilon}$ é um representante qualquer de u tal que $u_{\varepsilon}(\Omega) \subset \Omega^{\prime}$, para todo $\varepsilon \in \mathbf{I}$.

Finalizamos esta seção apresentando o conjunto $\mathcal{O}_{M}(\Omega ; \mathbb{R})$. Esse conjunto será importante no capítulo 2 .

Definição 1.3.16 Seja $\Omega$ um aberto de $\mathbb{R}^{n}$. Definimos

$$
\begin{gathered}
\mathcal{O}_{M}(\Omega ; \mathbb{R}):=\left\{f \in \mathscr{C}^{\infty}(\Omega ; \mathbb{R}) \mid \forall \alpha \in \mathbb{N}^{n}, \exists p \in \mathbb{N} e \exists c>0\right. \text { tais que } \\
\left.\left|\partial^{\alpha} f(x)\right| \leq c(1+|x|)^{-p}, \forall x \in \Omega\right\} .
\end{gathered}
$$

É claro que $\mathcal{O}_{M}\left(\mathbb{R}^{n} ; \mathbb{R}\right) \subset \mathcal{E}_{\tau}\left[\mathbb{R}^{n} ; \mathbb{R}\right]$, e assim, pode-se definir

$$
\begin{aligned}
i_{\mathcal{O}_{M}}: \mathcal{O}_{M}\left(\mathbb{R}^{n} ; \mathbb{R}\right) & \longrightarrow \mathcal{G}_{\tau}\left(\mathbb{R}^{n} ; \mathbb{R}\right) \\
f & \longmapsto(f)_{\varepsilon}+\mathcal{N}_{\tau}\left[\mathbb{R}^{n} ; \mathbb{R}\right]
\end{aligned}
$$


Note que $i_{\mathcal{O}_{M}}$ é injetiva, pois, se $(f)_{\varepsilon} \in \mathcal{N}_{\tau}\left[\mathbb{R}^{n} ; \mathbb{R}\right]$, então existem $N \in \mathbb{N}, c>0$ e $\eta \in \mathbf{I}$ tais que

$$
|f(x)| \leq c(1+|x|)^{N} \varepsilon, \quad \forall(\varepsilon, x) \in \mathbf{I}_{\eta} \times \mathbb{R}^{n} .
$$

Logo, fixado $a \in \mathbb{R}^{n}$, tem-se

$$
|f(a)| \leq c(1+|a|)^{N} \varepsilon, \quad \forall \varepsilon \in \mathbf{I}_{\eta}
$$

e portanto $f(a)=0$.

Proposição 1.3.17 Sejam $\Omega$ um aberto de $\mathbb{R}^{n}$, $\Omega^{\prime}$ um aberto convexo de $\mathbb{R}^{p}$, $v \in \mathcal{O}_{M}\left(\Omega^{\prime} ; \mathbb{R}\right) \quad$ e $\left(u_{\varepsilon}\right)_{\varepsilon} \in \mathcal{E}_{M}\left[\Omega ; \mathbb{R}^{p}\right] \quad$ tal que $u_{\varepsilon}(\Omega) \subset \Omega^{\prime}, \quad \forall \varepsilon \in \mathbf{I}$. São verdadeiras as seguintes afirmações:

1. $\left(v \circ u_{\varepsilon}\right)_{\varepsilon} \in \mathcal{E}_{M}[\Omega ; \mathbb{R}]$

2. se $\left(w_{\varepsilon}\right)_{\varepsilon} \in \mathcal{E}_{M}\left[\Omega ; \mathbb{R}^{p}\right]$ é tal que $\left(u_{\varepsilon}-w_{\varepsilon}\right)_{\varepsilon} \in \mathcal{N}\left[\Omega ; \mathbb{R}^{p}\right]$ e $w_{\varepsilon}(\Omega) \subset \Omega^{\prime}$, para todo $\varepsilon \in \mathbf{I}$, então

$$
\left(v \circ u_{\varepsilon}-v \circ w_{\varepsilon}\right)_{\varepsilon} \in \mathcal{N}[\Omega ; \mathbb{R}]
$$

Demonstração: Seja $\left(v_{\varepsilon}\right)_{\varepsilon}:=(v)_{\varepsilon}$. Então $\left(v_{\varepsilon}\right)_{\varepsilon} \in \mathcal{E}_{\tau}\left[\Omega^{\prime} ; \mathbb{R}\right]$. Logo o resultado segue da Proposição 1.3.14 (1.) e (2.).

Do resultado anterior podemos definir a seguinte composta.

Definição 1.3.18 Sejam $\Omega$ um aberto de $\mathbb{R}^{n}, \Omega^{\prime}$ um aberto convexo de $\mathbb{R}^{p}, u \in \mathcal{G}\left(\Omega ; \Omega^{\prime}\right)$ e $v \in \mathcal{O}_{M}\left(\Omega^{\prime} ; \mathbb{R}\right)$. A composta de u e $v$, denotada por $v$ ou, é o elemento de $\mathcal{G}(\Omega ; \mathbb{R})$ definido por

$$
v \circ u:=\left(v \circ u_{\varepsilon}\right)_{\varepsilon}+\mathcal{N}[\Omega ; \mathbb{R}]
$$

onde $\left(u_{\varepsilon}\right)_{\varepsilon}$ é um representante qualquer de u tal que $u_{\varepsilon}(\Omega) \subset \Omega^{\prime}$, para todo $\varepsilon \in \mathbf{I}$. 


\subsection{A álgebra $\widetilde{\mathcal{G}_{\tau}}\left(\Omega_{1} \times \Omega_{2} ; \mathbb{R}^{m}\right)$}

A álgebra $\widetilde{\mathcal{G}}_{\tau}\left(\Omega_{1} \times \Omega_{2} ; \mathbb{R}^{m}\right)$ que estudaremos nesta seção, apresenta características das duas álgebras apresentadas nas seções 1.2 e 1.3. Por apresentar, na segunda variável, uma condição similar à das aplicações generalizadas temperadas optamos por chamá-la de álgebra das aplicações generalizadas temperadas na segunda variável. Como na seção anterior destacamos o Teorema de Nulidade em $\widetilde{\mathcal{E}}_{\tau}\left[\Omega_{1} \times \Omega_{2} ; \mathbb{R}^{m}\right]$ (Teorema 1.4.5).

Definição 1.4.1 Sejam $\Omega_{1}$ e $\Omega_{2}$ abertos, respectivamente, de $\mathbb{R}^{p}$ e $\mathbb{R}^{q}$. Definimos

$$
\begin{aligned}
\widetilde{\mathcal{E}}_{\tau}\left[\Omega_{1} \times \Omega_{2} ; \mathbb{R}\right]:= & \left\{\left(u_{\varepsilon}\right)_{\varepsilon} \in \mathcal{E}\left[\Omega_{1} \times \Omega_{2} ; \mathbb{R}\right] \mid \forall K \subset \subset \Omega_{1}, \forall \alpha \in \mathbb{N}^{p+q}, \exists N \in \mathbb{N},\right. \\
& \exists c>0 e \exists \eta \in \mathbf{I} \text { tais que }\left|\partial^{\alpha} u_{\varepsilon}(x, y)\right| \leq c(1+|y|)^{N} \varepsilon^{-N}, \\
& \left.\forall(\varepsilon, x, y) \in \mathbf{I}_{\eta} \times K \times \Omega_{2}\right\} ; \\
\widetilde{\mathcal{N}}_{\tau}\left[\Omega_{1} \times \Omega_{2} ; \mathbb{R}\right]:=\{ & \left\{\left(u_{\varepsilon}\right)_{\varepsilon} \in \mathcal{E}\left[\Omega_{1} \times \Omega_{2} ; \mathbb{R}\right] \mid \forall K \subset \subset \Omega_{1}, \forall \alpha \in \mathbb{N}^{p+q}, \exists N \in \mathbb{N},\right. \\
& \forall r \in \mathbb{N}, \exists c>0 e \exists \eta \in \mathbf{I} \text { tais que } \\
& \left.\left|\partial^{\alpha} u_{\varepsilon}(x, y)\right| \leq c(1+|y|)^{N} \varepsilon^{r}, \quad \forall(\varepsilon, x, y) \in \mathbf{I}_{\eta} \times K \times \Omega_{2}\right\} .
\end{aligned}
$$

Um elemento de $\widetilde{\mathcal{E}}_{\tau}\left[\Omega_{1} \times \Omega_{2} ; \mathbb{R}\right]$ é chamado de elemento moderado temperado na segunda variável em $\Omega_{1} \times \Omega_{2}$ a valores em $\mathbb{R}$ e um elemento de $\widetilde{\mathcal{N}}_{\tau}\left[\Omega_{1} \times \Omega_{2} ; \mathbb{R}\right]$ é chamado de elemento nulo temperado na segunda variável em $\Omega_{1} \times \Omega_{2}$ a valores em $\mathbb{R}$.

Decorre diretamente da Definição 1.4 .1 que $\widetilde{\mathcal{E}}_{\tau}\left[\Omega_{1} \times \Omega_{2} ; \mathbb{R}\right]$ é uma $\mathbb{R}$-álgebra e que $\widetilde{\mathcal{N}}_{\tau}\left[\Omega_{1} \times \Omega_{2} ; \mathbb{R}\right]$ é um ideal de $\widetilde{\mathcal{E}}_{\tau}\left[\Omega_{1} \times \Omega_{2} ; \mathbb{R}\right]$. Logo, pode-se definir o seguinte.

Definição 1.4.2 Sejam $\Omega_{1}$ e $\Omega_{2}$ abertos, respectivamente, de $\mathbb{R}^{p}$ e $\mathbb{R}^{q}$. A álgebra das funções generalizadas temperadas na segunda variável de Colombeau é definida pelo espaço 
quociente

$$
\widetilde{\mathcal{G}}_{\tau}\left(\Omega_{1} \times \Omega_{2} ; \mathbb{R}\right):=\widetilde{\mathcal{E}}_{\tau}\left[\Omega_{1} \times \Omega_{2} ; \mathbb{R}\right] / \widetilde{\mathcal{N}}_{\tau}\left[\Omega_{1} \times \Omega_{2} ; \mathbb{R}\right]
$$

Um elemento de $\widetilde{\mathcal{G}}_{\tau}\left(\Omega_{1} \times \Omega_{2} ; \mathbb{R}\right)$ é chamado de função generalizada temperada na segunda variável em $\Omega_{1} \times \Omega_{2}$ a valores em $\mathbb{R}$.

Definição 1.4.3 Sejam $\Omega_{1}$ e $\Omega_{2}$ abertos, respectivamente, de $\mathbb{R}^{p}$ e $\mathbb{R}^{q}$ e $m \in \mathbb{N}$ com $m \geq 2$. Definimos

$$
\widetilde{\mathcal{G}}_{\tau}\left(\Omega_{1} \times \Omega_{2} ; \mathbb{R}^{m}\right):=\left(\widetilde{\mathcal{E}}_{\tau}\left[\Omega_{1} \times \Omega_{2} ; \mathbb{R}\right]\right)^{m} /\left(\widetilde{\mathcal{N}}_{\tau}\left[\Omega_{1} \times \Omega_{2} ; \mathbb{R}\right]\right)^{m}
$$

Um elemento de $\widetilde{\mathcal{G}}_{\tau}\left(\Omega_{1} \times \Omega_{2} ; \mathbb{R}^{m}\right)$ é chamado de aplicação generalizada temperada na segunda variável em $\Omega_{1} \times \Omega_{2}$ e a valores em $\mathbb{R}^{m}$.

Para facilitar a escrita escreveremos $\widetilde{\mathcal{E}}_{\tau}\left[\Omega_{1} \times \Omega_{2} ; \mathbb{R}^{m}\right]$ e $\widetilde{\mathcal{N}}_{\tau}\left[\Omega_{1} \times \Omega_{2} ; \mathbb{R}^{m}\right]$ em vez de $\left(\widetilde{\mathcal{E}}_{\tau}\left[\Omega_{1} \times \Omega_{2} ; \mathbb{R}\right]\right)^{m}$ e $\left(\widetilde{\mathcal{N}}_{\tau}\left[\Omega_{1} \times \Omega_{2} ; \mathbb{R}\right]\right)^{m}$, respectivamente.

É fácil verificar que a aplicação

$$
\begin{aligned}
\left(\widetilde{\mathcal{G}}_{\tau}\left(\Omega_{1} \times \Omega_{2} ; \mathbb{R}\right)\right)^{m} & \longrightarrow \widetilde{\mathcal{G}}_{\tau}\left(\Omega_{1} \times \Omega_{2} ; \mathbb{R}^{m}\right) \\
\left(u_{1_{\varepsilon}}+\widetilde{\mathcal{N}}_{\tau}\left[\Omega_{1} \times \Omega_{2} ; \mathbb{R}\right], \cdots, u_{m_{\varepsilon}}+\widetilde{\mathcal{N}}_{\tau}\left[\Omega_{1} \times \Omega_{2} ; \mathbb{R}\right]\right) & \longmapsto\left(u_{1_{\varepsilon}}, \cdots, u_{m_{\varepsilon}}\right)+\widetilde{\mathcal{N}}_{\tau}\left[\Omega_{1} \times \Omega_{2} ; \mathbb{R}^{m}\right],
\end{aligned}
$$

é um isomorfismo de álgebras. Portanto, para cada $m \in \mathbb{N}^{*}$, podemos identificar $\widetilde{\mathcal{G}}_{\tau}\left(\Omega_{1} \times \Omega_{2} ; \mathbb{R}^{m}\right) \operatorname{com}\left(\widetilde{\mathcal{G}}_{\tau}\left(\Omega_{1} \times \Omega_{2} ; \mathbb{R}\right)\right)^{m}$. Note ainda o seguinte.

Observação 1.4.4 Sejam $\Omega_{1} \quad e \quad \Omega_{2}$ abertos, respectivamente, de $\mathbb{R}^{p} \quad e \quad \mathbb{R}^{q} \quad e$ $\left(u_{\varepsilon}\right)_{\varepsilon} \in\left(\mathcal{E}\left[\Omega_{1} \times \Omega_{2} ; \mathbb{R}\right]\right)^{m}$. Tem-se

1. $\left(u_{\varepsilon}\right)_{\varepsilon} \in \widetilde{\mathcal{E}}_{\tau}\left[\Omega_{1} \times \Omega_{2} ; \mathbb{R}^{m}\right]$ se, e somente se, para quaisquer $K \subset \subset \Omega_{1}$ e $\alpha \in \mathbb{N}^{p+q}$, existem $N \in \mathbb{N}, c>0$ e $\eta \in \mathbf{I}$ tais que

$$
\left|\partial^{\alpha} u_{\varepsilon}(x, y)\right| \leq c(1+|y|)^{N} \varepsilon^{-N}, \quad \forall(\varepsilon, x, y) \in \mathbf{I}_{\eta} \times K \times \Omega_{2} ;
$$


2. $\left(u_{\varepsilon}\right)_{\varepsilon} \in \widetilde{\mathcal{N}}_{\tau}\left[\Omega_{1} \times \Omega_{2} ; \mathbb{R}^{m}\right]$ se, e somente se, para quaisquer $K \subset \subset \Omega_{1}$ e $\alpha \in \mathbb{N}^{p+q}$, existe $N \in \mathbb{N}$ tal que, para todo $r \in \mathbb{N}$, existem $c>0$ e $\eta \in \mathbf{I}$ tais que

$$
\left|\partial^{\alpha} u_{\varepsilon}(x, y)\right| \leq c(1+|y|)^{N} \varepsilon^{r}, \quad \forall(\varepsilon, x, y) \in \mathbf{I}_{\eta} \times K \times \Omega_{2}
$$

Note que, se $\Omega_{1}$ e $\Omega_{2}$ são abertos, respectivamente, de $\mathbb{R}^{p}$ e $\mathbb{R}^{q}$, então todo elemento $\left(u_{\varepsilon}\right)_{\varepsilon} \in \widetilde{\mathcal{E}}_{\tau}\left[\Omega_{1} \times \Omega_{2} ; \mathbb{R}^{m}\right]$ está associado a um único elemento $u \in \widetilde{\mathcal{G}}_{\tau}\left(\Omega_{1} \times \Omega_{2} ; \mathbb{R}^{m}\right)$ da seguinte forma

$$
u:=\left[\left(u_{\varepsilon}\right)_{\varepsilon}\right]=\left(u_{\varepsilon}\right)_{\varepsilon}+\widetilde{\mathcal{N}}_{\tau}\left[\Omega_{1} \times \Omega_{2} ; \mathbb{R}^{m}\right] .
$$

Nesse caso, dizemos que $\left(u_{\varepsilon}\right)_{\varepsilon} \in \widetilde{\mathcal{E}}_{\tau}\left[\Omega_{1} \times \Omega_{2} ; \mathbb{R}^{m}\right]$ é um representante de $u \in \widetilde{\mathcal{G}}_{\tau}\left(\Omega_{1} \times \Omega_{2} ; \mathbb{R}^{m}\right)$.

A partir do estudo das provas do Teorema de Nulidade em $\mathcal{E}_{M}\left[\Omega ; \mathbb{R}^{m}\right]$ e do Teorema de Nulidade em $\mathcal{E}_{\tau}\left[\Omega ; \mathbb{R}^{m}\right]$ (Teorema 1.2.5 e Teorema 1.3.7) obtivemos o seguinte resultado.

Teorema 1.4.5 (Teorema de Nulidade em $\widetilde{\mathcal{E}}_{\tau}\left[\Omega_{1} \times \Omega_{2} ; \mathbb{R}^{m}\right]$ ) Sejam $\Omega_{1}$ um aberto de $\mathbb{R}^{p}$, $\Omega_{2}$ um aberto de $\mathbb{R}^{q}$ tal que $\Omega_{2} \in \mathcal{A}^{*}\left(\mathbb{R}^{q}\right)$ e $\left(u_{\varepsilon}\right)_{\varepsilon} \in \widetilde{\mathcal{E}}_{\tau}\left[\Omega_{1} \times \Omega_{2} ; \mathbb{R}^{m}\right]$. São equivalentes as seguintes afirmações

(a) $\left(u_{\varepsilon}\right)_{\varepsilon} \in \widetilde{\mathcal{N}}_{\tau}\left[\Omega_{1} \times \Omega_{2} ; \mathbb{R}^{m}\right] ;$

(b) $\forall K \subset \subset \Omega_{1}, \exists N \in \mathbb{N}, \forall r \in \mathbb{N}, \exists c>0 e \exists \eta \in \mathbf{I}$ tais que

$$
\left|u_{\varepsilon}(x, y)\right| \leq c(1+|y|)^{N} \varepsilon^{r}, \quad \forall(\varepsilon, x, y) \in \mathbf{I}_{\eta} \times K \times \Omega_{2} .
$$

Demonstração: Segue da definição de $\widetilde{\mathcal{N}}_{\tau}\left[\Omega_{1} \times \Omega_{2} ; \mathbb{R}^{m}\right]$ que $(a)$ implica $(b)$. Para obtermos a implicação contrária basta que a mesma seja verdadeira no caso em que $m=1$.

Suponha $m=1$. 
Provaremos, por indução sobre $|\alpha|$, onde $\alpha \in \mathbb{N}^{p+q}$, que

$\forall K \subset \subset \Omega_{1}, \forall \alpha \in \mathbb{N}^{p+q}, \exists N \in \mathbb{N}, \forall r \in \mathbb{N}, \exists c>0$ e $\exists \eta \in \mathbf{I}$ tais que

$$
\left|\partial^{\alpha} u_{\varepsilon}(x, y)\right| \leq c(1+|y|)^{N} \varepsilon^{r}, \quad \forall(\varepsilon, x, y) \in \mathbf{I}_{\eta} \times K \times \Omega_{2} .
$$

É claro, por $(b)$, que, se $|\alpha|=0$, então (1.24) é verdadeira.

Suponha $\alpha=\left(\alpha_{1}, \cdots, \alpha_{p+q}\right) \in \mathbb{N}^{p+q}$ com $|\alpha|>0$ e suponha (1.24) verdadeira para todo $\gamma \in \mathbb{N}^{p+q} \operatorname{com}|\gamma|=|\alpha|-1$. De $|\alpha|>0$ existe $1 \leq j \leq p+q$ tal que $\alpha_{j} \neq 0$, e assim

$$
\partial^{\alpha} u_{\varepsilon}=\frac{\partial}{\partial x_{j}}\left(\frac{\partial^{|\alpha|-1} u_{\varepsilon}}{\partial x_{1}^{\alpha_{1}} \cdots \partial x_{j}^{\alpha_{j}-1} \cdots \partial x_{p+q}^{\alpha_{p+q}}}\right)=\frac{\partial v_{\varepsilon}}{\partial x_{j}}
$$

onde $v_{\varepsilon}:=\frac{\partial^{|\alpha|-1} u_{\varepsilon}}{\partial x_{1}^{\alpha_{1}} \cdots \partial x_{j}^{\alpha_{j}-1} \cdots \partial x_{p+q}^{\alpha_{p+q}}} \in \widetilde{\mathcal{E}}_{\tau}\left[\Omega_{1} \times \Omega_{2} ; \mathbb{R}\right]$, para todo $\varepsilon \in \mathbf{I}$.

Da hipótese de indução, tem-se

$$
\begin{gathered}
\forall K_{1} \subset \subset \Omega_{1}, \exists N \in \mathbb{N}, \forall s \in \mathbb{N}, \exists c>0 \text { e } \exists \eta \in \mathbf{I} \text { tais que } \\
\left|v_{\varepsilon}(x, y)\right| \leq c(1+|y|)^{N} \varepsilon^{s}, \quad \forall(\varepsilon, x, y) \in \mathbf{I}_{\eta} \times K_{1} \times \Omega_{2} .
\end{gathered}
$$

Seja $K \subset \subset \Omega_{1}$ e considere $V$ aberto de $\Omega_{1}$ tal que $K \subset V \subset \bar{V} \subset \subset \Omega_{1}$. Sejam $b:=\frac{1}{2} \min \left\{1, d\left(K, \Omega_{1} \backslash V\right)\right\} \quad$ e $\quad L:=K+\left\{x \in \mathbb{R}^{p}|| x \mid \leq b\right\} . \quad$ Então $K \subset L \subset \subset \Omega_{1}$.

Seja $r \in \mathbb{N}$ e denote $\frac{\partial}{\partial w_{i}}$ e $\frac{\partial^{2}}{\partial w_{i}^{2}}$ por $\partial_{i}$ e $\partial_{i}^{2}$, respectivamente, onde $w_{i}$ é a i-ésima coordenada de um ponto arbitrário de $\mathbb{R}^{p+q}$ e $1 \leq i \leq p+q$.

$\operatorname{De}\left(v_{\varepsilon}\right)_{\varepsilon} \in \widetilde{\mathcal{E}}_{\tau}\left[\Omega_{1} \times \Omega_{2} ; \mathbb{R}\right]$, existe $N_{1} \in \mathbb{N}$ com $N_{1}>1, c_{1}>0$ e $\eta_{1} \in \mathbf{I}$ tais que

$$
\left|\partial_{i}^{2} v_{\varepsilon}(x, y)\right| \leq c_{1}(1+|y|)^{N_{1}} \varepsilon^{-N_{1}}, \quad \forall(\varepsilon, x, y) \in \mathbf{I}_{\eta_{1}} \times L \times \Omega_{2} .
$$

De (1.25) para $K_{1}:=L$ e $s:=2 r+N_{1}$, existem $N \in \mathbb{N} \operatorname{com} N>N_{1}, c_{2}>c_{1}$ e $\eta_{2} \in \mathbf{I}$ $\operatorname{com} \eta_{2}<\eta_{1}$ tais que

$$
\left|v_{\varepsilon}(x, y)\right| \leq c_{2}(1+|y|)^{N} \varepsilon^{2 r+N_{1}}, \quad \forall(\varepsilon, x, y) \in \mathbf{I}_{\eta_{2}} \times L \times \Omega_{2}
$$

Como $\Omega_{2} \in \mathcal{A}^{*}\left(\mathbb{R}^{n}\right)$ existe $\nu>0$ como na Definição 1.3.5. substituindo $\Omega$ por $\Omega_{2}$.

Seja $\eta_{3}=\min \left\{\eta_{2}, b, \nu\right\}$ e fixemos $(\varepsilon, x, y) \in \mathbf{I}_{\eta_{3}} \times K \times \Omega_{2}$. Note que o segmento de extremidades $x$ e $x+\varepsilon^{r+N_{1}} e_{j}$ está contido em $L$, onde $e_{j}$ é o $j$-ésimo elemento da base 
canônica de $\mathbb{R}^{p}, 1 \leq j \leq p$, pois os elementos desse segmento são da forma $x+t \varepsilon^{r+N_{1}} e_{j}$ para $t \in[0,1]$. Observe também que o segmento de extremidades $y$ e $y+\varepsilon^{r+N_{1}} f_{k}$ ou o segmento de extremidades $y-\varepsilon^{r+N_{1}} f_{k}$ está contido em $\Omega_{2}$, onde $f_{k}$ é o $k$-ésimo elemento da base canônica de $\mathbb{R}^{q}, 1 \leq k \leq q$, pois os elementos desse segmento são da forma $y+t \varepsilon^{r+N_{1}} f_{k}$ ou $y-t \varepsilon^{r+N_{1}} f_{k}$ para $t \in[0,1]$.

Suponhamos, em primeiro lugar, $1 \leq i \leq p$. Como o segmento de extremidades $x$ e $x+\varepsilon^{r+N_{1}} e_{i}$ está contido em $L$ temos, usando o Polinômio de Taylor de ordem 1, que

$$
v_{\varepsilon}\left(\left(x+\varepsilon^{r+N_{1}} e_{i}, y\right)\right)=v_{\varepsilon}(x, y)+\partial_{i} v_{\varepsilon}(x, y) \varepsilon^{r+N_{1}}+\frac{1}{2} \partial_{i}^{2} v_{\varepsilon}(z, y)\left(\varepsilon^{r+N_{1}}\right)^{2}
$$

onde $z=x+\lambda \varepsilon^{r+N_{1}} e_{i}$ para algum $\left.\lambda \in\right] 0,1[$, ou seja

$$
\partial_{i} v_{\varepsilon}(x, y) \varepsilon^{r+N_{1}}=v_{\varepsilon}\left(\left(x+\varepsilon^{r+N_{1}} e_{i}, y\right)\right)-v_{\varepsilon}(x, y)-\frac{1}{2} \partial_{i}^{2} v_{\varepsilon}(z, y)\left(\varepsilon^{r+N_{1}}\right)^{2} .
$$

Suponhamos $p+1 \leq i \leq p+q$. Como o segmento de extremidades $y$ e $y+\varepsilon^{r+N_{1}} f_{i-p}$ ou o segmento de extremidades $y$ e $y-\varepsilon^{r+N_{1}} f_{i-p}$ está contido em $\Omega_{2}$ temos, usando o Polinômio de Taylor de ordem 1, que

$$
v_{\varepsilon}\left(\left(x, y \pm \varepsilon^{r+N_{1}} f_{i-p}\right)\right)=v_{\varepsilon}(x, y)+\partial_{i} v_{\varepsilon}(x, y) \varepsilon^{r+N_{1}}+\frac{1}{2} \partial_{i}^{2} v_{\varepsilon}(x, w)\left(\varepsilon^{r+N_{1}}\right)^{2}
$$

onde $w=y+\theta \varepsilon^{r+N_{1}} f_{i-p}$ para algum $\left.\theta \in\right] 0,1[$, ou seja

$$
\partial_{i} v_{\varepsilon}(x, y) \varepsilon^{r+N_{1}}=v_{\varepsilon}\left(\left(x, y \pm \varepsilon^{r+N_{1}} f_{i-p}\right)\right)-v_{\varepsilon}(x, y)-\frac{1}{2} \partial_{i}^{2} v_{\varepsilon}(x, w)\left(\varepsilon^{r+N_{1}}\right)^{2} .
$$

De (1.26), (1.27), (1.28) e (1.29) temos que

$$
\begin{aligned}
\left|\partial_{i} v_{\varepsilon}(x, y)\right| \varepsilon^{r+N_{1}} \leq c_{2}\left(1+|y|+\varepsilon^{r+N_{1}}\right)^{N} \varepsilon^{2 r+N_{1}}+c_{2}(1+|y|)^{N} \varepsilon^{2 r+N_{1}}+ \\
+\frac{1}{2} c_{1}\left(1+|y|+\varepsilon^{r+N_{1}}\right)^{N_{1}} \varepsilon^{-N_{1}}\left(\varepsilon^{r+N_{1}}\right)^{2}
\end{aligned}
$$

ou seja

$$
\begin{aligned}
\left|\partial_{i} v_{\varepsilon}(x, y)\right| & \leq c_{2}\left(1+|y|+\varepsilon^{r+N_{1}}\right)^{N} \varepsilon^{r}+c_{2}(1+|y|)^{N} \varepsilon^{r}+\frac{1}{2} c_{1}\left(1+|y|+\varepsilon^{r+N_{1}}\right)^{N_{1}} \varepsilon^{r} \\
& \leq c_{2}(2+|y|)^{N} \varepsilon^{r}+c_{2}(1+|y|)^{N} \varepsilon^{r}+\frac{1}{2} c_{1}(2+|y|)^{N_{1}} \varepsilon^{r} \\
& \leq c_{2}(2(1+|y|))^{N} \varepsilon^{r}+c_{2}(1+|y|)^{N} \varepsilon^{r}+\frac{1}{2} c_{2}(2(1+|y|))^{N_{1}} \varepsilon^{r} \\
& \leq c_{2}\left(2^{N}+1+2^{N_{1}-1}\right)(1+|y|)^{N} \varepsilon^{r} .
\end{aligned}
$$


Seja $c:=c_{2}\left(2^{N}+1+2^{N_{1}-1}\right)$. Então

$$
\left|\partial_{i} v_{\varepsilon}(x, y)\right| \leq c(1+|y|)^{N} \varepsilon^{r}, \quad \forall(\varepsilon, x, y) \in \mathbf{I}_{\eta_{3}} \times K \times \Omega_{2},
$$

o que prova (1.24).

Para apresentar a definição de derivada de aplicações moderadas temperadas na segunda variável é importante observar que, se $\Omega_{1}$ um aberto de $\mathbb{R}^{p}, \Omega_{2}$ é um aberto de $\mathbb{R}^{q}$, $\left(u_{\varepsilon}\right)_{\varepsilon} \in \widetilde{\mathcal{E}}_{\tau}\left[\Omega_{1} \times \Omega_{2} ; \mathbb{R}^{m}\right], \alpha \in \mathbb{N}^{p+q}$ e $\left(\partial^{\alpha} u_{\varepsilon}\right)_{\varepsilon} \in \mathcal{E}\left[\Omega_{1} \times \Omega_{2} ; \mathbb{R}^{m}\right]$ é definida por:

$$
\left(\partial^{\alpha} u_{\varepsilon}\right)_{\varepsilon}:(x, y) \in \Omega_{1} \times \Omega_{2} \longrightarrow \partial^{\alpha}\left(u_{\varepsilon}\right)(x, y) \in \mathbb{R}^{m}
$$

então $\left(\partial^{\alpha} u_{\varepsilon}\right)_{\varepsilon} \in \widetilde{\mathcal{E}}_{\tau}\left[\Omega_{1} \times \Omega_{2} ; \mathbb{R}^{m}\right]$. Assim das propriedades lineares das derivadas, decorre que a aplicação

$$
\begin{aligned}
\partial^{\alpha}: \widetilde{\mathcal{E}}_{\tau}\left[\Omega_{1} \times \Omega_{2} ; \mathbb{R}^{m}\right] & \longrightarrow \widetilde{\mathcal{E}}_{\tau}\left[\Omega_{1} \times \Omega_{2} ; \mathbb{R}^{m}\right] \\
\left(u_{\varepsilon}\right)_{\varepsilon} & \longmapsto\left(\partial^{\alpha} u_{\varepsilon}\right)_{\varepsilon}
\end{aligned}
$$

é um homomorfismo de $\mathbb{R}$-espaços vetoriais. Note ainda que $\partial^{\alpha}\left(\widetilde{\mathcal{E}}_{\tau}\left[\Omega_{1} \times \Omega_{2} ; \mathbb{R}^{m}\right]\right) \subset \widetilde{\mathcal{E}}_{\tau}\left[\Omega_{1} \times \Omega_{2} ; \mathbb{R}^{m}\right]$ e $\partial^{\alpha}\left(\widetilde{\mathcal{N}}_{\tau}\left[\Omega_{1} \times \Omega_{2} ; \mathbb{R}^{m}\right]\right) \subset \widetilde{\mathcal{N}}_{\tau}\left[\Omega_{1} \times \Omega_{2} ; \mathbb{R}^{m}\right]$. Logo, do Teorema do Homomorfismo, existe uma única aplicação $\mathbb{R}$-linear $\partial^{\alpha}: \widetilde{\mathcal{G}}_{\tau}\left(\Omega_{1} \times \Omega_{2} ; \mathbb{R}^{m}\right) \longrightarrow \widetilde{\mathcal{G}}_{\tau}\left(\Omega_{1} \times \Omega_{2} ; \mathbb{R}^{m}\right)$ tal que o diagrama

$$
\begin{array}{ccc}
\widetilde{\mathcal{E}}_{\tau}\left[\Omega_{1} \times \Omega_{2} ; \mathbb{R}^{m}\right] & \stackrel{\partial^{\alpha}}{\longrightarrow} & \widetilde{\mathcal{E}}_{\tau}\left[\Omega_{1} \times \Omega_{2} ; \mathbb{R}^{m}\right] \\
j \downarrow & j \downarrow \\
\widetilde{\mathcal{G}}_{\tau}\left(\Omega_{1} \times \Omega_{2} ; \mathbb{R}^{m}\right) & \stackrel{\partial^{\alpha}}{\longrightarrow} & \widetilde{\mathcal{G}}_{\tau}\left(\Omega_{1} \times \Omega_{2} ; \mathbb{R}^{m}\right)
\end{array}
$$

comuta, onde $j:\left(u_{\varepsilon}\right)_{\varepsilon} \in \widetilde{\mathcal{E}}_{\tau}\left[\Omega_{1} \times \Omega_{2} ; \mathbb{R}^{m}\right] \longmapsto\left(u_{\varepsilon}\right)_{\varepsilon}+\widetilde{\mathcal{N}}_{\tau}\left[\Omega_{1} \times \Omega_{2} ; \mathbb{R}^{m}\right]$. A partir desse fato, temos a seguinte definição.

Definição 1.4.6 Sejam $\Omega_{1}$ um aberto de $\mathbb{R}^{p}, \quad \Omega_{2}$ um aberto de $\mathbb{R}^{q}$, $u:=\left[\left(u_{\varepsilon}\right)_{\varepsilon}\right] \in \widetilde{\mathcal{G}}_{\tau}\left(\Omega_{1} \times \Omega_{2} ; \mathbb{R}^{m}\right)$ e $\alpha \in \mathbb{N}^{p+q}$. Chamamos de derivada parcial de ordem $\alpha$ de u o elemento $\partial^{\alpha} u:=\left[\left(\partial^{\alpha} u_{\varepsilon}\right)_{\varepsilon}\right] \in \widetilde{\mathcal{G}}_{\tau}\left(\Omega_{1} \times \Omega_{2} ; \mathbb{R}^{m}\right)$. 
A Definição 1.4.6 é compatível com a fórmula de Leibniz, ou seja, se $u, v \in \widetilde{\mathcal{G}}_{\tau}\left(\Omega_{1} \times \Omega_{2} ; \mathbb{R}\right)$ e $\alpha \in \mathbb{N}^{p+q}$, então tem-se

$$
\partial^{\alpha}(u v)=\sum_{0 \leq \beta \leq \alpha}\left(\begin{array}{l}
\alpha \\
\beta
\end{array}\right)\left(\partial^{\beta} u\right)\left(\partial^{\alpha-\beta} v\right)
$$

Para apresentar a composta de um elemento de $\mathcal{G}\left(\Omega ; \mathbb{R}^{p} \times \mathbb{R}^{q}\right)$ com um elemento de $\widetilde{\mathcal{G}_{\tau}}\left(\Omega^{\prime} \times \mathbb{R}^{q} ; \mathbb{R}^{m}\right)$, onde $\Omega$ é um aberto de $\mathbb{R}^{n}$ e $\Omega^{\prime}$ um aberto de $\mathbb{R}^{p}$ utilizaremos, como foi feito na seção 2, de um conjunto especial. Mais precisamente,

Definição 1.4.7 Sejam $\Omega$ um aberto de $\mathbb{R}^{n} \quad$ e $\Omega^{\prime}$ um aberto de $\mathbb{R}^{p}$. Denotamos por $\quad \widetilde{\mathcal{G}}_{*}\left(\Omega ; \Omega^{\prime} \times \mathbb{R}^{q}\right) \quad$ o conjunto das aplicações generalizadas $u:=\left(u_{1}, \cdots, u_{p}, u_{p+1}, \cdots, u_{p+q}\right) \in \mathcal{G}\left(\Omega ; \mathbb{R}^{p} \times \mathbb{R}^{q}\right) \quad$ tais $\quad$ que $\quad\left(u_{1}, \cdots, u_{p}\right) \in \mathcal{G}_{*}\left(\Omega ; \Omega^{\prime}\right)$.

Proposição 1.4.8 Sejam $\Omega$ um aberto de $\mathbb{R}^{n}, \Omega^{\prime}$ um aberto de $\mathbb{R}^{p}$. Se u $\in \widetilde{\mathcal{G}}_{*}\left(\Omega ; \Omega^{\prime} \times \mathbb{R}^{q}\right)$, então, todo representante $\left(u_{\varepsilon}\right)_{\varepsilon}$ de u satisfaz

$$
\forall K \subset \subset \Omega, \exists K^{\prime} \subset \subset \Omega^{\prime} e \exists \eta \in \mathbf{I} \text { tais que } g_{\varepsilon}(K) \subset K^{\prime}, \quad \forall \varepsilon \in \mathbf{I}_{\eta},
$$

onde $\left(g_{\varepsilon}\right)_{\varepsilon}:=\left(\left(u_{1_{\varepsilon}}, \cdots, u_{p_{\varepsilon}}\right)\right)_{\varepsilon} e\left(u_{\varepsilon}\right)_{\varepsilon}:=\left(\left(u_{1_{\varepsilon}}, u_{2_{\varepsilon}}, \cdots, u_{p_{\varepsilon}}, u_{(p+1)_{\varepsilon}}, u_{(p+2)_{\varepsilon}}, \cdots, u_{(p+q)_{\varepsilon}}\right)\right)_{\varepsilon}$.

Demonstração: $\quad$ Sejam $\quad\left(u_{\varepsilon}\right)_{\varepsilon}:=\left(\left(u_{1_{\varepsilon}}, u_{2_{\varepsilon}}, \cdots, u_{p_{\varepsilon}}, u_{(p+1)_{\varepsilon}}, u_{(p+2)_{\varepsilon}}, \cdots, u_{(p+q)_{\varepsilon}}\right)\right)_{\varepsilon} \mathrm{e}$ $\left(v_{\varepsilon}\right)_{\varepsilon}:=\left(\left(v_{1_{\varepsilon}}, v_{2_{\varepsilon}}, \cdots, v_{p_{\varepsilon}}, v_{(p+1)_{\varepsilon}}, v_{(p+2)_{\varepsilon}}, \cdots, v_{(p+q)_{\varepsilon}}\right)\right)_{\varepsilon}$ representantes de $u$. Defina $\left(g_{\varepsilon}\right)_{\varepsilon}:=\left(\left(u_{1_{\varepsilon}}, \cdots, u_{p_{\varepsilon}}\right)\right)_{\varepsilon}$ e $\left(h_{\varepsilon}\right)_{\varepsilon}:=\left(\left(v_{1_{\varepsilon}}, \cdots, v_{p_{\varepsilon}}\right)\right)_{\varepsilon}$. Note que, se $\left(u_{\varepsilon}\right)_{\varepsilon}$ satisfaz $(1.30)$, então $\left(g_{\varepsilon}\right)_{\varepsilon}$ satisfaz $(1.6)$, e como $\left(h_{\varepsilon}\right)_{\varepsilon}$ é um representante de $\left(g_{\varepsilon}\right)_{\varepsilon}+\mathcal{N}\left[\Omega ; \mathbb{R}^{p}\right]$ temos que $\left(h_{\varepsilon}\right)_{\varepsilon}$ também satisfaz (1.6) ([Fer]). Logo $\left(v_{\varepsilon}\right)_{\varepsilon}$ verifica $(1.30)$.

Proposição 1.4.9 Sejam $\Omega$ um aberto de $\mathbb{R}^{n}, \quad \Omega^{\prime}$ um aberto de $\mathbb{R}^{p}$, $\left(v_{\varepsilon}\right)_{\varepsilon} \in \widetilde{\mathcal{E}}_{\tau}\left[\Omega^{\prime} \times \mathbb{R}^{q} ; \mathbb{R}^{m}\right] e\left(u_{\varepsilon}\right)_{\varepsilon}:=\left(\left(u_{1_{\varepsilon}}, \cdots, u_{p_{\varepsilon}}, u_{(p+1)_{\varepsilon}}, \cdots, u_{(p+q)_{\varepsilon}}\right)\right)_{\varepsilon} \in \mathcal{E}_{M}\left[\Omega ; \mathbb{R}^{p} \times \mathbb{R}^{q}\right]$ tal que 
(i) $\forall K \subset \subset \Omega, \exists K^{\prime} \subset \subset \Omega^{\prime}$ e $\eta \in \mathbf{I}$ tais que $g_{\varepsilon}(K) \subset K^{\prime}, \quad \forall \varepsilon \in \mathbf{I}_{\eta}$,

onde $\left(g_{\varepsilon}\right)_{\varepsilon}:=\left(\left(u_{1_{\varepsilon}}, \cdots, u_{p_{\varepsilon}}\right)\right)_{\varepsilon}$. São verdadeiras as seguintes afirmações:

1. $\left(v_{\varepsilon} \circ u_{\varepsilon}\right)_{\varepsilon} \in \mathcal{E}_{M}\left[\Omega ; \mathbb{R}^{m}\right]$;

2. se $\left(w_{\varepsilon}\right)_{\varepsilon} \in \mathcal{E}_{M}\left[\Omega ; \mathbb{R}^{p} \times \mathbb{R}^{q}\right]$ é tal que $\left(w_{\varepsilon}-u_{\varepsilon}\right)_{\varepsilon} \in \mathcal{N}\left[\Omega ; \mathbb{R}^{p} \times \mathbb{R}^{q}\right]$, então

$$
\left(v_{\varepsilon} \circ u_{\varepsilon}-v_{\varepsilon} \circ w_{\varepsilon}\right)_{\varepsilon} \in \mathcal{N}\left[\Omega ; \mathbb{R}^{m}\right]
$$

3. se $\left(f_{\varepsilon}\right)_{\varepsilon} \in \widetilde{\mathcal{E}}_{\tau}\left[\Omega^{\prime} \times \mathbb{R}^{q} ; \mathbb{R}^{m}\right]$ é tal que $\left(f_{\varepsilon}-v_{\varepsilon}\right)_{\varepsilon} \in \widetilde{\mathcal{N}}_{\tau}\left[\Omega^{\prime} \times \mathbb{R}^{q} ; \mathbb{R}^{m}\right]$, então

$$
\left(v_{\varepsilon} \circ u_{\varepsilon}-f_{\varepsilon} \circ u_{\varepsilon}\right)_{\varepsilon} \in \mathcal{N}\left[\Omega ; \mathbb{R}^{m}\right]
$$

4. se $\left(w_{\varepsilon}\right)_{\varepsilon}$ é como em 2. e $\left(f_{\varepsilon}\right)_{\varepsilon}$ é como em 3., então

$$
\left(v_{\varepsilon} \circ u_{\varepsilon}-f_{\varepsilon} \circ w_{\varepsilon}\right)_{\varepsilon} \in \mathcal{N}\left[\Omega ; \mathbb{R}^{m}\right]
$$

Demonstração: Como foi observado na prova da Proposição 1.3.10, podemos admitir, sem perda de generalidade, $m=1$ e que para obter 1 . basta provar que, se $\alpha \in \mathbb{N}^{n}$, então, para todo $K \subset \subset \Omega$ e para quaisquer $\beta \in \mathbb{N}^{p+q}$ e $\gamma \in \mathbb{N}^{n}$ com $|\beta|,|\gamma| \leq|\alpha|$, existem $N \in \mathbb{N}$, $c>0$ e $\eta \in \mathbf{I}$ tais que

$$
\begin{gathered}
\left|\partial^{\gamma} u_{\varepsilon}(x)\right| \leq c \varepsilon^{-N}, \quad \forall(\varepsilon, x) \in \mathbf{I}_{\eta} \times K ; \\
\left|\left(\partial^{\beta} v_{\varepsilon}\right) \circ u_{\varepsilon}(x)\right| \leq c \varepsilon^{-N}, \quad \forall(\varepsilon, x) \in \mathbf{I}_{\eta} \times K .
\end{gathered}
$$

Sejam $\alpha \in \mathbb{N}^{n}$ e $K \subset \subset \Omega$. De $\left(u_{\varepsilon}\right)_{\varepsilon} \in \mathcal{E}_{M}\left[\Omega ; \mathbb{R}^{p} \times \mathbb{R}^{q}\right]$, existem $N_{1} \in \mathbb{N}, c_{1}>0$ e $\eta \in \mathbf{I}$ tais que

$$
\left|\partial^{\gamma} u_{\varepsilon}(x)\right| \leq c_{1} \varepsilon^{-N_{1}}, \quad \forall(\varepsilon, x) \in \mathbf{I}_{\eta_{1}} \times K, \forall \gamma \in \mathbb{N}^{n} \operatorname{com}|\gamma| \leq|\alpha| .
$$

De $(i)$, existem $K^{\prime} \subset \subset \Omega^{\prime}$ e $\eta_{2} \in \mathbf{I}$ com $\eta_{2}<\eta_{1}$ tais que $g_{\varepsilon}(K) \subset K^{\prime}$, para $\varepsilon \in \mathbf{I}_{\eta_{2}}$.

De $\left(v_{\varepsilon}\right)_{\varepsilon} \in \widetilde{\mathcal{E}}_{\tau}\left[\Omega^{\prime} \times \mathbb{R}^{q} ; \mathbb{R}^{m}\right]$, existem $N_{2} \in \mathbb{N}$ com $N_{2}>N_{1}, c_{2}>c_{1}$ e $\eta_{3} \in \mathbf{I} \mathrm{com}$ $\eta_{3}<\eta_{2}$ tais que $\left|\partial^{\beta} v_{\varepsilon}(z, y)\right| \leq c_{3}(1+|y|)^{N_{2}} \varepsilon^{-N_{2}}, \quad \forall(\varepsilon, z, y) \in \mathbf{I}_{\eta_{3}} \times K^{\prime} \times \mathbb{R}^{q}, \forall \beta \in \mathbb{N}^{p+q} \operatorname{com}|\beta| \leq|\alpha|$. 
Portanto, se $\left(h_{\varepsilon}\right)_{\varepsilon}:=\left(\left(u_{(p+1)_{\varepsilon}}, \cdots, u_{(p+q)_{\varepsilon}}\right)\right)_{\varepsilon}$, temos, para todo $\beta \in \mathbb{N}^{p+q} \operatorname{com}|\beta| \leq|\alpha|$, que

$$
\left|\partial^{\beta} v_{\varepsilon}\left(u_{\varepsilon}(x)\right)\right|=\left|\partial^{\beta} v_{\varepsilon}\left(g_{\varepsilon}(x), h_{\varepsilon}(x)\right)\right| \leq c_{3}\left(1+\left|h_{\varepsilon}(x)\right|\right)^{N_{2}} \varepsilon^{-N_{2}}, \quad \forall(\varepsilon, x) \in \mathbf{I}_{\eta_{3}} \times K .
$$

Seja $\beta \in \mathbb{N}^{p+q} \operatorname{com}|\beta| \leq|\alpha|$. De (1.33) e (1.34) temos, para $(\varepsilon, x) \in \mathbf{I}_{\eta_{3}} \times K$, que

$$
\begin{aligned}
\left|\partial^{\beta} v_{\varepsilon}\left(u_{\varepsilon}(x)\right)\right| & \leq c_{3}\left(1+\left|h_{\varepsilon}(x)\right|\right)^{N_{2}} \varepsilon^{-N_{2}} \\
& \leq c_{3}\left(1+\left|u_{\varepsilon}(x)\right|\right)^{N_{2}} \varepsilon^{-N_{2}} \\
& \leq c_{3}\left(1+c_{1} \varepsilon^{-N_{1}}\right)^{N_{2}} \varepsilon^{-N_{2}} \\
& \leq c_{3}\left(1+c_{1}\right)^{N_{2}} \varepsilon^{-N_{1} N_{2}} \varepsilon^{-N_{2}} \\
& \leq c_{3}\left(1+c_{1}\right)^{N_{2}} \varepsilon^{-\left(N_{2}^{2}+N_{2}\right)}
\end{aligned}
$$

o que, juntamente com (1.33), provam (1.31) e (1.32).

Seja $K \subset \subset \Omega$.

Para 2. utilizaremos o Teorema de Nulidade em $\mathcal{E}_{M}\left[\Omega ; \mathbb{R}^{p} \times \mathbb{R}^{q}\right]$ (Teorema 1.2.5).

Suponha que $\left(w_{\varepsilon}\right)_{\varepsilon}:=\left(\left(w_{1_{\varepsilon}}, \cdots, w_{p_{\varepsilon}}, w_{(p+1)_{\varepsilon}}, \cdots, w_{(p+q)_{\varepsilon}}\right)\right)_{\varepsilon} \quad$ e $\quad$ considere $\left(g_{1_{\varepsilon}}\right)_{\varepsilon}:=\left(\left(w_{1_{\varepsilon}}, \cdots, w_{p_{\varepsilon}}\right)\right)_{\varepsilon}$ e $\quad\left(h_{1_{\varepsilon}}\right)_{\varepsilon}:=\left(\left(w_{(p+1)_{\varepsilon}}, \cdots, w_{(p+q)_{\varepsilon}}\right)\right)_{\varepsilon}$.

Da Proposição 1.4.8, existem $K^{\prime} \subset \subset \Omega^{\prime}$ e $\eta_{4} \in \mathbf{I}$ tais que

$$
g_{\varepsilon}(K) \subset K^{\prime} \quad \text { e } \quad g_{1_{\varepsilon}}(K) \subset K^{\prime}, \quad \forall \varepsilon \in \mathbf{I}_{\eta_{4}}
$$

Seja $V$ um aberto de $\mathbb{R}^{p}$ tal que $K^{\prime} \subset V \subset \bar{V} \subset \subset \Omega^{\prime}$. Considere $b:=\frac{1}{2} \min \left\{1, d\left(K^{\prime}, \Omega^{\prime} \backslash V\right)\right\} \quad$ e $L:=K^{\prime}+\left\{x \in \mathbb{R}^{p}|| x \mid \leq b\right\}$.

$\operatorname{De}\left(v_{\varepsilon}\right)_{\varepsilon} \in \widetilde{\mathcal{E}}_{\tau}\left[\Omega^{\prime} \times \mathbb{R}^{q} ; \mathbb{R}^{m}\right]$ e $\left(u_{\varepsilon}\right)_{\varepsilon},\left(w_{\varepsilon}\right)_{\varepsilon} \in \mathcal{E}_{M}\left[\Omega ; \mathbb{R}^{p} \times \mathbb{R}^{q}\right]$, existem $N_{4} \in \mathbb{N}, c_{4}>0$ e $\eta_{5} \in \mathbf{I} \operatorname{com} \eta_{5}<\eta_{4}$ tais que

$$
\begin{gathered}
\left|\partial^{\gamma} v_{\varepsilon}(z, y)\right| \leq c_{4}(1+|y|)^{N_{4}} \varepsilon^{-N_{4}}, \quad \forall(\varepsilon, z, y) \in \mathbf{I}_{\eta_{5}} \times L \times \mathbb{R}^{q}, \forall \gamma \in \mathbb{N}^{p+q} \operatorname{com}|\gamma|=1 ; \\
\left|u_{\varepsilon}(x)\right| \leq c_{4} \varepsilon^{-N_{4}}, \quad \forall(\varepsilon, x) \in \mathbf{I}_{\eta_{5}} \times K \\
\left|w_{\varepsilon}(x)\right| \leq c_{4} \varepsilon^{-N_{4}}, \quad \forall(\varepsilon, x) \in \mathbf{I}_{\eta_{5}} \times K .
\end{gathered}
$$


Seja $r \in \mathbb{N}$.

$\operatorname{De}\left(n_{\varepsilon}\right)_{\varepsilon}:=\left(w_{\varepsilon}-u_{\varepsilon}\right)_{\varepsilon}=\left(g_{\varepsilon}-g_{1_{\varepsilon}}, h_{\varepsilon}-h_{1_{\varepsilon}}\right)=:\left(\left(n_{1_{\varepsilon}}, n_{2_{\varepsilon}}\right)\right)_{\varepsilon} \in \mathcal{N}\left[\Omega ; \mathbb{R}^{p} \times \mathbb{R}^{q}\right]$, para $k:=r+N_{4}^{2}+N_{4}+1$, existem $c_{5}>c_{4}$ e $\eta_{6} \in \mathbf{I}$ com $\eta_{6}<\eta_{5}$ tais que

$$
\left|n_{\varepsilon}(x)\right| \leq c_{5} \varepsilon^{k}, \quad \forall(\varepsilon, x) \in \mathbf{I}_{\eta_{6}} \times K .
$$

Seja $\eta_{7}:=\min \left\{\eta_{6}, \frac{b}{c_{5}}\right\}$ e fixemos $(\varepsilon, x) \in \mathbf{I}_{\eta_{7}} \times K$.

Note que o segmento de extremidades $u_{\varepsilon}(x)$ e $w_{\varepsilon}(x)$ está contido em $L$, pois os elementos desse segmento são da forma

$$
w_{\varepsilon}(x)-t n_{\varepsilon}(x)=\left(g_{1_{\varepsilon}}(x)+t n_{1_{\varepsilon}}(x), h_{1_{\varepsilon}}(x)+t n_{2_{\varepsilon}}(x)\right), \text { para } t \in[0,1]
$$

e, por (1.35) e (1.39), temos que $g_{1_{\varepsilon}}(x) \in K^{\prime}$ e

$$
\left|t n_{1_{\varepsilon}}(x)\right| \leq\left|n_{1_{\varepsilon}}(x)\right| \leq\left|n_{\varepsilon}(x)\right| \leq c_{5} \varepsilon^{k} \leq c_{5} \varepsilon<b \text {, para todo } t \in[0,1] .
$$

Dessa forma, usando o Teorema do Valor Médio, existe $\sigma \in] 0,1[$ tal que

$$
\begin{aligned}
\left|v_{\varepsilon}\left(u_{\varepsilon}(x)\right)-v_{\varepsilon}\left(w_{\varepsilon}(x)\right)\right| & =\left|\left\langle u_{\varepsilon}(x)-w_{\varepsilon}(x), \nabla v_{\varepsilon}\left(w_{\varepsilon}(x)+\sigma n_{\varepsilon}(x)\right)\right\rangle\right| \\
& \leq\left|n_{\varepsilon}(x)\right|\left|\nabla v_{\varepsilon}\left(w_{\varepsilon}(x)+\sigma n_{\varepsilon}(x)\right)\right|,
\end{aligned}
$$

onde $\langle$,$\rangle denota o produto interno usual de \mathbb{R}^{p+q}$. Portanto, de (1.36), (1.37), (1.38) e (1.39), temos que

$$
\begin{aligned}
\left|v_{\varepsilon}\left(u_{\varepsilon}(x)\right)-v_{\varepsilon}\left(w_{\varepsilon}(x)\right)\right| & \leq\left|n_{\varepsilon}(x)\right|\left|\nabla v_{\varepsilon}\left(w_{\varepsilon}(x)+\sigma n_{\varepsilon}(x)\right)\right| \\
& \leq c_{5} \varepsilon^{k} c_{4}(p+q)\left(1+\left|h_{1_{\varepsilon}}(x)+\sigma n_{2_{\varepsilon}}(x)\right|\right)^{N_{4}} \varepsilon^{-N_{4}} \\
& \leq c_{5} \varepsilon^{k} c_{4}(p+q)\left(1+\left|h_{1_{\varepsilon}}(x)\right|+\left|\sigma n_{2_{\varepsilon}}(x)\right|\right)^{N_{4}} \varepsilon^{-N_{4}} \\
& \leq c_{5} \varepsilon^{k} c_{4}(p+q)\left(1+\left|w_{\varepsilon}(x)\right|+\left|n_{\varepsilon}(x)\right|\right)^{N_{4}} \varepsilon^{-N_{4}} \\
& \leq c_{5} \varepsilon^{k} c_{4}(p+q)\left(1+\left|w_{\varepsilon}(x)\right|+\left|u_{\varepsilon}(x)\right|+\left|w_{\varepsilon}(x)\right|\right)^{N_{4}} \varepsilon^{-N_{4}} \\
& \leq c_{5} \varepsilon^{k} c_{4}(p+q)\left(1+3 c_{4} \varepsilon^{-N_{4}}\right)^{N_{4}} \varepsilon^{-N_{4}} \\
& \leq c_{5} \varepsilon^{k} c_{4}(p+q)\left(1+3 c_{4}\right)^{N_{4}} \varepsilon^{-N_{4}^{2}-N_{4}} \\
& \leq(p+q) c_{5}^{2}\left(1+3 c_{5}\right)^{N_{4}} \varepsilon^{k-N_{4}^{2}-N_{4}} .
\end{aligned}
$$


Seja $c:=(p+q) c_{5}^{2}\left(1+3 c_{5}\right)^{N_{4}}$. Então

$$
\left|v_{\varepsilon}\left(u_{\varepsilon}(x)\right)-v_{\varepsilon}\left(w_{\varepsilon}(x)\right)\right| \leq c \varepsilon^{r}, \quad \forall(\varepsilon, x) \in \mathbf{I}_{\eta_{7}} \times K
$$

Para 3. provaremos que, para todo $s \in \mathbb{N}$, existem $c>0$ e $\eta \in \mathbf{I}$ tais que

$$
\left|v_{\varepsilon}\left(u_{\varepsilon}(x)\right)-f_{\varepsilon}\left(u_{\varepsilon}(x)\right)\right| \leq c \varepsilon^{s}, \quad \forall(\varepsilon, x) \in \mathbf{I}_{\eta} \times K,
$$

e, do Teorema de Nulidade em $\mathcal{E}_{M}[\Omega ; \mathbb{R}]$ (Teorema 1.2.5), seguirá que

$$
\left(v_{\varepsilon} \circ u_{\varepsilon}-f_{\varepsilon} \circ u_{\varepsilon}\right)_{\varepsilon} \in \mathcal{N}[\Omega ; \mathbb{R}] .
$$

Sejam $N_{4}, c_{4}$ e $\eta_{5}$ como em (1.37) e (1.38).

De $\left(m_{\varepsilon}\right)_{\varepsilon}:=\left(v_{\varepsilon}-f_{\varepsilon}\right)_{\varepsilon} \in \widetilde{\mathcal{N}}_{\tau}\left[\Omega^{\prime} \times \mathbb{R}^{q} ; \mathbb{R}\right]$, existe $N_{5} \in \mathbb{N}$ com $N_{5}>N_{4}$ tal que, para todo $k \in \mathbb{N}$, existem $c_{k}>c_{4}$ e $\eta_{k} \in \mathbf{I}$ com $\eta_{k}<\eta_{5}$ tais que

$$
\left|m_{\varepsilon}(z, y)\right| \leq c_{k}(1+|y|)^{N_{5}} \varepsilon^{k}, \quad \forall(\varepsilon, z, y) \in \mathbf{I}_{\eta_{k}} \times K^{\prime} \times \mathbb{R}^{q}
$$

Considere $N:=N_{5}^{2}$ e seja $s \in \mathbb{N}$. Então, para $k:=s+N$, existem $c_{6}:=c_{k}$ e $\eta_{8}:=\eta_{k}$ como em (1.40), isto é

$$
\left|m_{\varepsilon}(z, y)\right| \leq c_{6}(1+|y|)^{N_{5}} \varepsilon^{k}, \quad \forall(\varepsilon, z, y) \in \mathbf{I}_{\eta_{8}} \times K^{\prime} \times \mathbb{R}^{q}
$$

De (1.35), (1.37) e (1.41) temos que

$$
\begin{aligned}
\left|v_{\varepsilon}\left(u_{\varepsilon}(x)\right)-f_{\varepsilon}\left(u_{\varepsilon}(x)\right)\right| & =\left|m_{\varepsilon}\left(u_{\varepsilon}(x)\right)\right| \\
& =\left|m_{\varepsilon}\left(g_{\varepsilon}(x), h_{\varepsilon}(x)\right)\right| \\
& \leq c_{6}\left(1+\left|h_{\varepsilon}(x)\right|\right)^{N^{5}} \varepsilon^{k} \\
& \leq c_{6}\left(1+\left|u_{\varepsilon}(x)\right|\right)^{N^{5}} \varepsilon^{k} \\
& \leq c_{6}\left(1+c_{4} \varepsilon^{-N_{4}}\right)^{N_{5}} \varepsilon^{k} \\
& \leq c_{6}\left(\varepsilon^{-N_{4}}+c_{4} \varepsilon^{-N_{4}}\right)^{N_{5}} \varepsilon^{k} \\
& \leq c_{6}\left(1+c_{4}\right)^{N_{5}} \varepsilon^{-N_{4} N_{5}} \varepsilon^{k} \\
& \leq c_{6}\left(1+c_{6}\right)^{N_{5}} \varepsilon^{k-N} .
\end{aligned}
$$


Seja $c:=c_{6}\left(1+c_{6}\right)^{N_{5}}$. Então

$$
\left|v_{\varepsilon}\left(u_{\varepsilon}(x)\right)-f_{\varepsilon}\left(u_{\varepsilon}(x)\right)\right| \leq c \varepsilon^{s}, \quad \forall(\varepsilon, x) \in \mathbf{I}_{\eta_{8}} \times K .
$$

Finalmente para 4. basta observar que

$$
v_{\varepsilon} \circ u_{\varepsilon}-f_{\varepsilon} \circ w_{\varepsilon}=\left(v_{\varepsilon} \circ u_{\varepsilon}-v_{\varepsilon} \circ w_{\varepsilon}\right)+\left(v_{\varepsilon} \circ w_{\varepsilon}-f_{\varepsilon} \circ w_{\varepsilon}\right),
$$

usar 2. e observar que 3. é verdadeira se $\left(u_{\varepsilon}\right)_{\varepsilon}$ for substituído por $\left(w_{\varepsilon}\right)_{\varepsilon}$.

A partir da Proposição 1.4.9 temos a seguinte definição.

Definição 1.4.10 Sejam $\Omega$ um aberto de $\mathbb{R}^{n}$ e $\Omega^{\prime}$ um aberto de $\mathbb{R}^{p}, u \in \widetilde{\mathcal{G}}_{*}\left(\Omega ; \Omega^{\prime} \times \mathbb{R}^{q}\right)$ e $v \in \widetilde{\mathcal{G}}_{\tau}\left(\Omega^{\prime} \times \mathbb{R}^{q} ; \mathbb{R}^{m}\right)$. A composta de u e $v$, denotada por $v \circ u$, é o elemento de $\mathcal{G}\left(\Omega ; \mathbb{R}^{m}\right)$ definido por

$$
v \circ u:=\left(v_{\varepsilon} \circ u_{\varepsilon}\right)_{\varepsilon}+\mathcal{N}\left[\Omega ; \mathbb{R}^{m}\right]
$$

onde $\left(u_{\varepsilon}\right)_{\varepsilon}$ é um representante qualquer de $u$ e $\left(v_{\varepsilon}\right)_{\varepsilon}$ é um representante qualquer de $v$.

Finalizamos esta seção "comparando"os conjuntos $\mathcal{E}_{\tau}\left[\Omega_{1} \times \Omega_{2} ; \mathbb{R}^{m}\right], \widetilde{\mathcal{E}}_{\tau}\left[\Omega_{1} \times \Omega_{2} ; \mathbb{R}^{m}\right]$ e $\mathcal{E}_{M}\left[\Omega_{1} \times \Omega_{2} ; \mathbb{R}^{m}\right]$ e os conjuntos $\mathcal{N}_{\tau}\left[\Omega_{1} \times \Omega_{2} ; \mathbb{R}^{m}\right], \widetilde{\mathcal{N}}_{\tau}\left[\Omega_{1} \times \Omega_{2} ; \mathbb{R}^{m}\right]$ e $\mathcal{N}\left[\Omega_{1} \times \Omega_{2} ; \mathbb{R}^{m}\right]$.

Proposição 1.4.11 Sejam $\Omega_{1}$ um aberto de $\mathbb{R}^{p}$ e $\Omega_{2}$ um aberto do $\mathbb{R}^{q}$. Então

1. $\mathcal{E}_{\tau}\left[\Omega_{1} \times \Omega_{2} ; \mathbb{R}^{m}\right] \subset \widetilde{\mathcal{E}}_{\tau}\left[\Omega_{1} \times \Omega_{2} ; \mathbb{R}^{m}\right] \subset \mathcal{E}_{M}\left[\Omega_{1} \times \Omega_{2} ; \mathbb{R}^{m}\right] ;$

2. $\mathcal{N}_{\tau}\left[\Omega_{1} \times \Omega_{2} ; \mathbb{R}^{m}\right] \subset \widetilde{\mathcal{N}}_{\tau}\left[\Omega_{1} \times \Omega_{2} ; \mathbb{R}^{m}\right] \subset \mathcal{N}\left[\Omega_{1} \times \Omega_{2} ; \mathbb{R}^{m}\right]$.

Demonstração: Decorre da definição de cada conjunto.

A partir da Proposição 1.4.11 (1.) podemos definir uma aplicação de $\mathcal{G}_{\tau}\left(\Omega_{1} \times \Omega_{2} ; \mathbb{R}^{m}\right)$ em $\widetilde{\mathcal{G}}_{\tau}\left(\Omega_{1} \times \Omega_{2} ; \mathbb{R}^{m}\right)$ e uma aplicação de $\widetilde{\mathcal{G}}_{\tau}\left(\Omega_{1} \times \Omega_{2} ; \mathbb{R}^{m}\right)$ em $\mathcal{G}\left(\Omega_{1} \times \Omega_{2} ; \mathbb{R}^{m}\right)$. Mais precisamente: 


$$
\begin{aligned}
\Psi_{1}: \mathcal{G}_{\tau}\left(\Omega_{1} \times \Omega_{2} ; \mathbb{R}^{m}\right) & \longrightarrow \widetilde{\mathcal{G}}_{\tau}\left(\Omega_{1} \times \Omega_{2} ; \mathbb{R}^{m}\right) \\
\left(u_{\varepsilon}\right)_{\varepsilon}+\mathcal{N}_{\tau}\left[\Omega_{1} \times \Omega_{2} ; \mathbb{R}^{m}\right] & \longmapsto\left(u_{\varepsilon}\right)_{\varepsilon}+\widetilde{\mathcal{N}}_{\tau}\left[\Omega_{1} \times \Omega_{2} ; \mathbb{R}^{m}\right] ; \\
\Psi_{2}: \widetilde{\mathcal{G}}_{\tau}\left(\Omega_{1} \times \Omega_{2} ; \mathbb{R}^{m}\right) & \longrightarrow \mathcal{G}\left(\Omega_{1} \times \Omega_{2} ; \mathbb{R}^{m}\right) \\
\left(u_{\varepsilon}\right)_{\varepsilon}+\widetilde{\mathcal{N}}_{\tau}\left[\Omega_{1} \times \Omega_{2} ; \mathbb{R}^{m}\right] & \longmapsto\left(u_{\varepsilon}\right)_{\varepsilon}+\mathcal{N}\left[\Omega_{1} \times \Omega_{2} ; \mathbb{R}^{m}\right]
\end{aligned}
$$

Convém observar que as aplicações $\Psi_{1}$ e $\Psi_{2}$, como mostra o exemplo a seguir, podem não ser injetoras.

Exemplo 1.4.12 Seja $\varphi \in \mathscr{C}^{\infty}(\mathbb{R} ; \mathbb{R})$ como no Exemplo 1.3.13.

1. se $\left(u_{\varepsilon}\right)_{\varepsilon}$ é definida por

$$
u_{\varepsilon}(x, y)=\varphi\left(x-\varepsilon^{-1}\right), \quad \forall(\varepsilon, x, y) \in \mathbf{I} \times \mathbb{R} \times \mathbb{R},
$$

então $\left(u_{\varepsilon}\right)_{\varepsilon} \in \mathcal{E}_{\tau}[\mathbb{R} \times \mathbb{R} ; \mathbb{R}],\left(u_{\varepsilon}\right)_{\varepsilon} \in \widetilde{\mathcal{N}}_{\tau}[\mathbb{R} \times \mathbb{R} ; \mathbb{R}] e\left(u_{\varepsilon}\right)_{\varepsilon} \notin \mathcal{N}_{\tau}[\mathbb{R} \times \mathbb{R} ; \mathbb{R}] ;$

2. se $\left(v_{\varepsilon}\right)_{\varepsilon}$ é definida por

$$
v_{\varepsilon}(x, y)=e^{x} \varphi\left(y-\varepsilon^{-1}\right), \quad \forall(\varepsilon, x, y) \in \mathbf{I} \times \mathbb{R} \times \mathbb{R},
$$

então $\left(v_{\varepsilon}\right)_{\varepsilon} \in \widetilde{\mathcal{E}}_{\tau}[\mathbb{R} \times \mathbb{R} ; \mathbb{R}],\left(v_{\varepsilon}\right)_{\varepsilon} \in \mathcal{N}[\mathbb{R} \times \mathbb{R} ; \mathbb{R}]$ e $\left(v_{\varepsilon}\right)_{\varepsilon} \notin \widetilde{\mathcal{N}}_{\tau}[\mathbb{R} \times \mathbb{R} ; \mathbb{R}]$.

De fato, basta usar o Exemplo 1.3 .13 e observar que $|x| \leq|(x, y)|,|y| \leq|(x, y)|$ e que a função $f(x)=e^{x}$ restrita a compactos de $\mathbb{R}$ tem valor máximo e valor mínimo.

\subsection{As álgebras $\mathcal{G}\left(\left[t_{0},+\infty\left[; \mathbb{R}^{m}\right)\right.\right.$ e $\mathcal{G}_{\tau}\left(\left[t_{0},+\infty\left[; \mathbb{R}^{m}\right)\right.\right.$}

Os resultados aqui encontrados serão utilizados na última seção deste trabalho. Assim como foi feito nas seções anteriores, apresentamos, para cada uma das álgebras aqui estudadas, um teorema de nulidade (Teorema 1.5.5 e Teorema 1.5.6).

Neste capítulo, e em todo o trabalho, utilizaremos a seguinte notação: 
- $\mathscr{C}^{\infty}\left(\left[t_{0},+\infty[; \mathbb{R}):=\left\{\varphi \in \mathbb{R}^{\left[t_{0},+\infty[\right.} \mid \exists s>0\right.\right.\right.$ e $\exists \psi \in \mathscr{C}^{\infty}(] t_{0}-s,+\infty[; \mathbb{R})$ tais que $\left.\varphi=\left.\psi\right|_{\left[t_{0},+\infty[\right.}\right\}$,

onde $t_{0} \in \mathbb{R}$.

Definição 1.5.1 Seja $t_{0} \in \mathbb{R}$. Definimos

$$
\begin{aligned}
& \mathcal{E}\left[\left[t_{0},+\infty[; \mathbb{R}]:=\left(\mathscr { C } ^ { \infty } \left(\left[t_{0},+\infty[; \mathbb{R})\right)^{\mathbf{I}} ;\right.\right.\right.\right. \\
& \mathcal{E}_{M}\left[\left[t_{0},+\infty[; \mathbb{R}]:=\left\{( u _ { \varepsilon } ) _ { \varepsilon } \in \mathcal { E } \left[\left[t_{0},+\infty[; \mathbb{R}] \mid \forall t_{1}>t_{0}, \forall p \in \mathbb{N}, \exists N \in \mathbb{N}, \exists c>0 e\right.\right.\right.\right.\right. \\
& \left.\exists \eta \in \mathbf{I} \text { tais que }\left|\frac{d^{p} u_{\varepsilon}}{d t^{p}}(t)\right| \leq c \varepsilon^{-N}, \quad \forall(\varepsilon, t) \in \mathbf{I}_{\eta} \times\left[t_{0}, t_{1}\right]\right\} ; \\
& \mathcal{N}\left[\left[t_{0},+\infty[; \mathbb{R}]:=\left\{( u _ { \varepsilon } ) _ { \varepsilon } \in \mathcal { E } \left[\left[t_{0},+\infty[; \mathbb{R}] \mid \forall t_{1}>t_{0}, \forall p \in \mathbb{N}, \forall r \in \mathbb{N}, \exists c>0 e\right.\right.\right.\right.\right. \\
& \left.\exists \eta \in \mathbf{I} \text { tais que }\left|\frac{d^{p} u_{\varepsilon}}{d t^{p}}(t)\right| \leq c \varepsilon^{r}, \quad \forall(\varepsilon, t) \in \mathbf{I}_{\eta} \times\left[t_{0}, t_{1}\right]\right\} ; \\
& \mathcal{E}_{\tau}\left[\left[t_{0},+\infty[; \mathbb{R}]:=\left\{( u _ { \varepsilon } ) _ { \varepsilon } \in \mathcal { E } \left[\left[t_{0},+\infty[; \mathbb{R}] \mid \forall p \in \mathbb{N}, \exists N \in \mathbb{N}, \exists c>0 \text { e } \exists \eta \in \mathbf{I}\right.\right.\right.\right.\right. \\
& \text { tais que }\left|\frac{d^{p} u_{\varepsilon}}{d t^{p}}(t)\right| \leq c(1+|t|)^{N} \varepsilon^{-N}, \quad \forall(\varepsilon, t) \in \mathbf{I}_{\eta} \times\left[t_{0},+\infty[\} ;\right. \\
& \mathcal{N}_{\tau}\left[\left[t_{0},+\infty[; \mathbb{R}]:=\left\{( u _ { \varepsilon } ) _ { \varepsilon } \in \mathcal { E } \left[\left[t_{0},+\infty[; \mathbb{R}] \mid \forall p \in \mathbb{N}, \exists N \in \mathbb{N}, \forall r \in \mathbb{N}, \exists c>0 e \exists \eta \in \mathbf{I}\right.\right.\right.\right.\right. \\
& \text { tais que }\left|\frac{d^{p} u_{\varepsilon}}{d t^{p}}(t)\right| \leq c(1+|t|)^{N} \varepsilon^{r}, \quad \forall(\varepsilon, t) \in \mathbf{I}_{\eta} \times\left[t_{0},+\infty[\}\right. \text {. }
\end{aligned}
$$

Um elemento de $\mathcal{E}_{M}\left[\left[t_{0},+\infty[; \mathbb{R}]\right.\right.$ é chamado de função moderada em $\left[t_{0},+\infty[a\right.$ valores em $\mathbb{R}$ e um elemento de $\mathcal{N}\left[\left[t_{0},+\infty[; \mathbb{R}]\right.\right.$ é chamado de função nula em $\left[t_{0},+\infty[\right.$ a valores em $\mathbb{R}$.

Um elemento de $\mathcal{E}_{\tau}\left[\left[t_{0},+\infty[; \mathbb{R}]\right.\right.$ é chamado de função moderada temperada em $\left[t_{0},+\infty\left[\right.\right.$ a valores em $\mathbb{R}$ e um elemento de $\mathcal{N}_{\tau}\left[\left[t_{0},+\infty[; \mathbb{R}]\right.\right.$ é chamado de função nula temperada em $\left[t_{0},+\infty[\right.$ a valores em $\mathbb{R}$.

Os conjuntos $\mathcal{E}_{M}\left[\left[t_{0},+\infty[; \mathbb{R}]\right.\right.$ e $\mathcal{E}_{\tau}\left[\left[t_{0},+\infty[; \mathbb{R}]\right.\right.$ munidos das operações usuais de soma e produto de funções e produto de número real por função são $\mathbb{R}$-álgebras. Decorre 
diretamente da Definição 1.5 .1 que $\mathcal{N}\left[\left[t_{0},+\infty[; \mathbb{R}]\right.\right.$ é um ideal de $\mathcal{E}_{M}\left[\left[t_{0},+\infty[; \mathbb{R}]\right.\right.$ e $\mathcal{N}_{\tau}\left[\left[t_{0},+\infty[; \mathbb{R}]\right.\right.$ é um ideal de $\mathcal{E}_{\tau}\left[\left[t_{0},+\infty[; \mathbb{R}]\right.\right.$. Assim, pode-se definir o seguinte.

Definição 1.5.2 Seja $t_{0} \in \mathbb{R}$. Definimos

$$
\begin{gathered}
\mathcal{G}\left(\left[t_{0},+\infty[; \mathbb{R}):=\mathcal{E}_{M}\left[\left[t_{0},+\infty[; \mathbb{R}] / \mathcal{N}\left[\left[t_{0},+\infty[; \mathbb{R}]\right.\right.\right.\right.\right.\right. \\
\mathcal{G}_{\tau}\left(\left[t_{0},+\infty[; \mathbb{R}):=\mathcal{E}_{\tau}\left[\left[t_{0},+\infty[; \mathbb{R}] / \mathcal{N}_{\tau}\left[\left[t_{0},+\infty[; \mathbb{R}]\right.\right.\right.\right.\right.\right.
\end{gathered}
$$

Um elemento de $\mathcal{G}\left(\left[t_{0},+\infty[; \mathbb{R})\right.\right.$ é chamado função generalizada em $\left[t_{0},+\infty[a\right.$ valores em $\mathbb{R}$ e um elemento de $\mathcal{G}_{\tau}\left(\left[t_{0},+\infty[; \mathbb{R})\right.\right.$ é chamado função generalizada temperada em $\left[t_{0},+\infty[\right.$ a valores em $\mathbb{R}$.

Definição 1.5.3 Sejam $t_{0} \in \mathbb{R}$ e $m \in \mathbb{N}$ com $m \geq 2$. Definimos

$$
\begin{gathered}
\mathcal{G}\left(\left[t_{0},+\infty\left[; \mathbb{R}^{m}\right):=\left(\mathcal { E } _ { M } \left[\left[t_{0},+\infty[; \mathbb{R}]\right)^{m} /\left(\mathcal { N } \left[\left[t_{0},+\infty[; \mathbb{R}]\right)^{m} ;\right.\right.\right.\right.\right.\right. \\
\mathcal{G}_{\tau}\left(\left[t_{0},+\infty\left[; \mathbb{R}^{m}\right):=\left(\mathcal { E } _ { \tau } \left[\left[t_{0},+\infty[; \mathbb{R}]\right)^{m} /\left(\mathcal { N } _ { \tau } \left[\left[t_{0},+\infty[; \mathbb{R}]\right)^{m}\right.\right.\right.\right.\right.\right.
\end{gathered}
$$

Um elemento de $\mathcal{G}\left(\left[t_{0},+\infty\left[; \mathbb{R}^{m}\right)\right.\right.$ é chamado de aplicação generalizada em $\left[t_{0},+\infty[\right.$ a valores em $\mathbb{R}^{m}$ e um elemento de $\mathcal{G}_{\tau}\left(\left[t_{0},+\infty\left[; \mathbb{R}^{m}\right)\right.\right.$ é chamado de aplicação generalizada temperada em $\left[t_{0},+\infty\left[\right.\right.$ a valores em $\mathbb{R}^{m}$.

Para facilitar a escrita escreveremos $\mathcal{E}_{M}\left[\left[t_{0},+\infty\left[; \mathbb{R}^{m}\right], \quad \mathcal{N}\left[\left[t_{0},+\infty\left[; \mathbb{R}^{m}\right]\right.\right.\right.\right.$, $\mathcal{E}_{\tau}\left[\left[t_{0},+\infty\left[; \mathbb{R}^{m}\right]\right.\right.$ e $\mathcal{N}_{\tau}\left[\Omega ; \mathbb{R}^{m}\right]$ em vez de $\left(\mathcal{E}_{M}\left[\left[t_{0},+\infty[; \mathbb{R}]\right)^{m}, \quad\left(\mathcal{N}\left[\left[t_{0},+\infty[; \mathbb{R}]\right)^{m}\right.\right.\right.\right.$, $\left(\mathcal{E}_{\tau}\left[\left[t_{0},+\infty[; \mathbb{R}]\right)^{m}\right.\right.$ e $\left(\mathcal{N}_{\tau}\left[\left[t_{0},+\infty[; \mathbb{R}]\right)^{m}\right.\right.$, respectivamente.

Note que as funções

$$
\begin{aligned}
\left(\mathcal { G } \left(\left[t_{0},+\infty[; \mathbb{R})\right)^{m}\right.\right. & \longrightarrow \mathcal{G}\left(\left[t_{0},+\infty\left[; \mathbb{R}^{m}\right)\right.\right. \\
\left(u_{1_{\varepsilon}}+\mathcal{N}\left[\left[t_{0},+\infty[; \mathbb{R}], \cdots, u_{m_{\varepsilon}}+\mathcal{N}\left[\left[t_{0},+\infty[; \mathbb{R}]\right)\right.\right.\right.\right. & \longmapsto\left(u_{1_{\varepsilon}}, \cdots, u_{m_{\varepsilon}}\right)+\mathcal{N}\left[\left[t_{0},+\infty\left[; \mathbb{R}^{m}\right] ;\right.\right.
\end{aligned}
$$




$$
\begin{aligned}
\left(\mathcal { G } _ { \tau } \left(\left[t_{0},+\infty[; \mathbb{R})\right)^{m}\right.\right. & \longrightarrow \mathcal{G}_{\tau}\left(\left[t_{0},+\infty\left[; \mathbb{R}^{m}\right)\right.\right. \\
\left(u_{1_{\varepsilon}}+\mathcal{N}_{\tau}\left[\left[t_{0},+\infty[; \mathbb{R}], \cdots, u_{m_{\varepsilon}}+\mathcal{N}_{\tau}\left[\left[t_{0},+\infty[; \mathbb{R}]\right)\right.\right.\right.\right. & \longmapsto\left(u_{1_{\varepsilon}}, \cdots, u_{m_{\varepsilon}}\right)+\mathcal{N}_{\tau}\left[\left[t_{0},+\infty\left[; \mathbb{R}^{m}\right],\right.\right.
\end{aligned}
$$

são isomorfismos de álgebras. Assim, para todo $m \in \mathbb{N}^{*}$, podemos identificar $\mathcal{G}\left(\left[t_{0},+\infty\left[; \mathbb{R}^{m}\right) \operatorname{com}\left(\mathcal{G}\left(\left[t_{0},+\infty[; \mathbb{R})\right)^{m}\right.\right.\right.\right.$ e $\mathcal{G}_{\tau}\left(\left[t_{0},+\infty\left[; \mathbb{R}^{m}\right) \operatorname{com}\left(\mathcal{G}_{\tau}\left(\left[t_{0},+\infty[; \mathbb{R})\right)^{m}\right.\right.\right.\right.$.

Além disso, observe o seguinte.

Observação 1.5.4 Sejam $t_{0} \in \mathbb{R} e\left(u_{\varepsilon}\right)_{\varepsilon} \in\left(\mathcal{E}\left[\left[t_{0},+\infty[; \mathbb{R}]\right)^{m}\right.\right.$. Tem-se

1. $\left(u_{\varepsilon}\right)_{\varepsilon} \in \mathcal{E}_{M}\left[\left[t_{0},+\infty\left[; \mathbb{R}^{m}\right]\right.\right.$ se, e somente se, para quaisquer $t_{1}>t_{0}$ e $p \in \mathbb{N}$, existem $N \in \mathbb{N}$, c $>0$ e $\eta \in \mathbf{I}$ tais que

$$
\left|\frac{d^{p} u_{\varepsilon}}{d t^{p}}(t)\right| \leq c \varepsilon^{-N}, \quad \forall(\varepsilon, t) \in \mathbf{I}_{\eta} \times\left[t_{0}, t_{1}\right] ;
$$

2. $\left(u_{\varepsilon}\right)_{\varepsilon} \in \mathcal{N}\left[\left[t_{0},+\infty\left[; \mathbb{R}^{m}\right]\right.\right.$ se, e somente se, para quaisquer $t_{1}>t_{0}, p \in \mathbb{N}$ e $r \in \mathbb{N}$, existem $c>0$ e $\eta \in \mathbf{I}$ tais que

$$
\left|\frac{d^{p} u_{\varepsilon}}{d t^{p}}(t)\right| \leq c \varepsilon^{r}, \quad \forall(\varepsilon, t) \in \mathbf{I}_{\eta} \times\left[t_{0}, t_{1}\right] ;
$$

3. $\left(u_{\varepsilon}\right)_{\varepsilon} \in \mathcal{E}_{\tau}\left[\left[t_{0},+\infty\left[; \mathbb{R}^{m}\right]\right.\right.$ se, e somente se, para qualquer $p \in \mathbb{N}$, existem $N \in \mathbb{N}, c>0$ e $\eta \in \mathbf{I}$ tais que

$$
\left|\frac{d^{p} u_{\varepsilon}}{d t^{p}}(t)\right| \leq c(1+|t|)^{N} \varepsilon^{-N}, \quad \forall(\varepsilon, t) \in \mathbf{I}_{\eta} \times\left[t_{0},+\infty[;\right.
$$

4. $\left(u_{\varepsilon}\right)_{\varepsilon} \in \mathcal{N}_{\tau}\left[\left[t_{0},+\infty\left[; \mathbb{R}^{m}\right]\right.\right.$ se, e somente se, para qualquer $p \in \mathbb{N}$, existe $N \in \mathbb{N}$ tal que, para todo $r \in \mathbb{N}$, existem $c>0$ e $\eta \in \mathbf{I}$ tais que

$$
\left|\frac{d^{p} u_{\varepsilon}}{d t^{p}}(t)\right| \leq c(1+|t|)^{N} \varepsilon^{r}, \quad \forall(\varepsilon, t) \in \mathbf{I}_{\eta} \times\left[t_{0},+\infty[.\right.
$$

Convém notar que, se $t_{0} \in \mathbb{R}$, então todo $\left(u_{\varepsilon}\right)_{\varepsilon} \in \mathcal{E}_{M}\left[\left[t_{0},+\infty\left[; \mathbb{R}^{m}\right]\right.\right.$ está associado a um único elemento $u \in \mathcal{G}\left(\left[t_{0},+\infty\left[; \mathbb{R}^{m}\right)\right.\right.$ da seguinte forma

$$
u:=\left[\left(u_{\varepsilon}\right)_{\varepsilon}\right]=\left(u_{\varepsilon}\right)_{\varepsilon}+\mathcal{N}\left[\left[t_{0},+\infty\left[; \mathbb{R}^{m}\right] .\right.\right.
$$


Nesse caso, dizemos que $\left(u_{\varepsilon}\right)_{\varepsilon} \in \mathcal{E}_{M}\left[\left[t_{0},+\infty\left[; \mathbb{R}^{m}\right]\right.\right.$ é um representante de $u \in \mathcal{G}\left(\left[t_{0},+\infty\left[; \mathbb{R}^{m}\right)\right.\right.$. Além disso, todo $\left(u_{\varepsilon}\right)_{\varepsilon} \in \mathcal{E}_{\tau}\left[\left[t_{0},+\infty\left[; \mathbb{R}^{m}\right]\right.\right.$ também está associado a um único elemento $u \in \mathcal{G}_{\tau}\left(\left[t_{0},+\infty\left[; \mathbb{R}^{m}\right)\right.\right.$ da seguinte forma

$$
u:=\left[\left(u_{\varepsilon}\right)_{\varepsilon}\right]=\left(u_{\varepsilon}\right)_{\varepsilon}+\mathcal{N}_{\tau}\left[\left[t_{0},+\infty\left[; \mathbb{R}^{m}\right]\right.\right.
$$

Nesse caso, dizemos que $\left(u_{\varepsilon}\right)_{\varepsilon} \in \mathcal{E}_{\tau}\left[\left[t_{0},+\infty\left[; \mathbb{R}^{m}\right]\right.\right.$ é um representante de $u \in \mathcal{G}_{\tau}\left(\left[t_{0},+\infty\left[; \mathbb{R}^{m}\right)\right.\right.$.

A partir do estudo das provas do Teorema de Nulidade em $\mathcal{E}_{M}\left[\Omega ; \mathbb{R}^{m}\right]$ e do Teorema de Nulidade em $\mathcal{E}_{\tau}\left[\Omega ; \mathbb{R}^{m}\right]$ (Teorema 1.2 .5 e Teorema 1.3.7) obtivemos o Teorema 1.5 .5 e o Teorema 1.5.6 que são, respectivamente, o Teorema de Nulidade em $\mathcal{E}_{M}\left[\left[t_{0},+\infty[; \mathbb{R}]\right.\right.$ e o Teorema de Nulidade em $\mathcal{E}_{\tau}\left[\left[t_{0},+\infty[; \mathbb{R}]\right.\right.$.

Teorema 1.5.5 (Teorema de Nulidade em $\mathcal{E}_{M}\left[\left[t_{0},+\infty\left[; \mathbb{R}^{m}\right]\right.\right.$ ) Sejam $t_{0} \in \mathbb{R} \quad e$ $\left(u_{\varepsilon}\right)_{\varepsilon} \in \mathcal{E}_{M}\left[\left[t_{0},+\infty\left[; \mathbb{R}^{m}\right]\right.\right.$. São equivalentes as seguintes afirmações:

(a) $\left(u_{\varepsilon}\right)_{\varepsilon} \in \mathcal{N}\left[\left[t_{0},+\infty\left[; \mathbb{R}^{m}\right]\right.\right.$

(b) $\forall t_{1}>t_{0}, \forall r \in \mathbb{N}, \exists c>0$ e $\eta \in \mathbf{I}$ tais que

$$
\left|u_{\varepsilon}(t)\right| \leq c \varepsilon^{r}, \quad \forall(\varepsilon, t) \in \mathbf{I} \times\left[t_{0}, t_{1}\right]
$$

Demonstração: Prova análoga à do Teorema 1.2.5.

Teorema 1.5.6 (Teorema de Nulidade em $\mathcal{E}_{\tau}\left[\left[t_{0},+\infty\left[; \mathbb{R}^{m}\right]\right.\right.$ ) Sejam $t_{0} \in \mathbb{R} \quad e$ $\left(u_{\varepsilon}\right)_{\varepsilon} \in \mathcal{N}_{\tau}\left[\left[t_{0},+\infty\left[; \mathbb{R}^{m}\right]\right.\right.$. São equivalentes as seguintes afirmações:

(a) $\left(u_{\varepsilon}\right)_{\varepsilon} \in \mathcal{N}_{\tau}\left[\left[t_{0},+\infty\left[; \mathbb{R}^{m}\right] ;\right.\right.$

(b) $\exists N \in \mathbb{N}, \forall r \in \mathbb{N}, \exists c>0$ e $\eta \in \mathbf{I}$ tais que

$$
\left|u_{\varepsilon}(t)\right| \leq c(1+|x|)^{N} \varepsilon^{r}, \forall(\varepsilon, t) \in \mathbf{I} \times\left[t_{0},+\infty[.\right.
$$


Demonstração: Prova análoga à do Teorema 1.3.7.

Na Definição 1.5.8 apresentaremos a composta de um elemento de $\mathcal{G}_{\tau}\left(\left[t_{0},+\infty\left[; \mathbb{R}^{n}\right)\right.\right.$ com um elemento de $\mathcal{G}_{\tau}\left(\mathbb{R}^{n} ; \mathbb{R}^{m}\right)$, e na Definição 1.5.10 apresentaremos a composta de um elemento de $\mathcal{G}\left(\left[t_{0},+\infty\left[; \mathbb{R}^{n}\right)\right.\right.$ com um elemento de $\mathcal{G}_{\tau}\left(\left[t_{0},+\infty\left[; \mathbb{R}^{m}\right)\right.\right.$. Para isto utilizaremos a Proposição 1.5 .7 e a Proposição 1.5.9, respectivamente.

Proposição 1.5.7 Sejam $t_{0} \in \mathbb{R},\left(v_{\varepsilon}\right)_{\varepsilon} \in \mathcal{E}_{\tau}\left[\mathbb{R}^{n} ; \mathbb{R}^{m}\right] e\left(u_{\varepsilon}\right)_{\varepsilon} \in \mathcal{E}_{\tau}\left[\left[t_{0},+\infty\left[; \mathbb{R}^{n}\right]\right.\right.$. Então

1. $\left(v_{\varepsilon} \circ u_{\varepsilon}\right)_{\varepsilon} \in \mathcal{E}_{\tau}\left[\left[t_{0},+\infty\left[; \mathbb{R}^{m}\right]\right.\right.$

2. se $\left(w_{\varepsilon}\right)_{\varepsilon} \in \mathcal{E}_{\tau}\left[\left[t_{0},+\infty\left[; \mathbb{R}^{n}\right]\right.\right.$ é tal que $\left(w_{\varepsilon}-u_{\varepsilon}\right)_{\varepsilon} \in \mathcal{N}_{\tau}\left[\left[t_{0},+\infty\left[; \mathbb{R}^{n}\right]\right.\right.$, então

$$
\left(v_{\varepsilon} \circ u_{\varepsilon}-v_{\varepsilon} \circ w_{\varepsilon}\right)_{\varepsilon} \in \mathcal{N}_{\tau}\left[\left[t_{0},+\infty\left[; \mathbb{R}^{m}\right] ;\right.\right.
$$

3. se $\left(f_{\varepsilon}\right)_{\varepsilon} \in \mathcal{E}_{\tau}\left[\mathbb{R}^{n} ; \mathbb{R}^{m}\right]$ é tal que $\left(f_{\varepsilon}-v_{\varepsilon}\right)_{\varepsilon} \in \mathcal{N}_{\tau}\left[\mathbb{R}^{n} ; \mathbb{R}^{m}\right]$, então

$$
\left(v_{\varepsilon} \circ u_{\varepsilon}-f_{\varepsilon} \circ u_{\varepsilon}\right)_{\varepsilon} \in \mathcal{N}_{\tau}\left[\left[t_{0},+\infty\left[; \mathbb{R}^{m}\right] ;\right.\right.
$$

4. se $\left(w_{\varepsilon}\right)_{\varepsilon}$ é como em 2. e $\left(f_{\varepsilon}\right)_{\varepsilon}$ é como em 3., então $\left(v_{\varepsilon} \circ u_{\varepsilon}-f_{\varepsilon} \circ w_{\varepsilon}\right)_{\varepsilon} \in \mathcal{E}_{\tau}\left[\left[t_{0},+\infty\left[; \mathbb{R}^{m}\right]\right.\right.$.

Demonstração: Prova análoga à da Proposição 1.3.10.

A partir da Proposição 1.5.7 temos a seginte definição.

Definição 1.5.8 Sejam $t_{0} \in \mathbb{R}, v \in \mathcal{G}_{\tau}\left(\mathbb{R}^{n} ; \mathbb{R}^{m}\right)$ e $u \in \mathcal{G}_{\tau}\left(\left[t_{0},+\infty\left[; \mathbb{R}^{n}\right)\right.\right.$. A composta de $u$ e $v$, denotada por $v \circ u$, é o elemento de $\mathcal{G}_{\tau}\left(\left[t_{0},+\infty\left[; \mathbb{R}^{m}\right)\right.\right.$ definido por

$$
v \circ u:=\left(v_{\varepsilon} \circ u_{\varepsilon}\right)_{\varepsilon}+\mathcal{N}_{\tau}\left[\left[t_{0},+\infty\left[; \mathbb{R}^{m}\right],\right.\right.
$$

onde $\left(u_{\varepsilon}\right)_{\varepsilon}$ é um representante qualquer de u e $\left(v_{\varepsilon}\right)_{\varepsilon}$ é um representante qualquer de $v$. 
Proposição 1.5.9 Sejam $t_{0} \in \mathbb{R},\left(v_{\varepsilon}\right)_{\varepsilon} \in \mathcal{E}_{\tau}\left[\mathbb{R}^{n} ; \mathbb{R}^{m}\right]$ e $\left(u_{\varepsilon}\right)_{\varepsilon} \in \mathcal{E}_{M}\left[\left[t_{0},+\infty\left[; \mathbb{R}^{n}\right]\right.\right.$. Então

1. $\left(v_{\varepsilon} \circ u_{\varepsilon}\right)_{\varepsilon} \in \mathcal{E}_{M}\left[\left[t_{0},+\infty\left[; \mathbb{R}^{m}\right] ;\right.\right.$

2. se $\left(w_{\varepsilon}\right)_{\varepsilon} \in \mathcal{E}_{M}\left[\left[t_{0},+\infty\left[; \mathbb{R}^{n}\right]\right.\right.$ é tal que $\left(w_{\varepsilon}-u_{\varepsilon}\right)_{\varepsilon} \in \mathcal{N}\left[\left[t_{0},+\infty\left[; \mathbb{R}^{n}\right]\right.\right.$, então

$$
\left(v_{\varepsilon} \circ u_{\varepsilon}-v_{\varepsilon} \circ w_{\varepsilon}\right)_{\varepsilon} \in \mathcal{N}\left[\left[t_{0},+\infty\left[; \mathbb{R}^{m}\right] ;\right.\right.
$$

3. se $\left(f_{\varepsilon}\right)_{\varepsilon} \in \mathcal{E}_{\tau}\left[\mathbb{R}^{n} ; \mathbb{R}^{m}\right]$ é tal que $\left(f_{\varepsilon}-v_{\varepsilon}\right)_{\varepsilon} \in \mathcal{N}_{\tau}\left[\mathbb{R}^{n} ; \mathbb{R}^{m}\right]$, então

$$
\left(v_{\varepsilon} \circ u_{\varepsilon}-f_{\varepsilon} \circ u_{\varepsilon}\right)_{\varepsilon} \in \mathcal{N}\left[\left[t_{0},+\infty\left[; \mathbb{R}^{m}\right]\right.\right.
$$

4. se $\left(w_{\varepsilon}\right)_{\varepsilon}$ é como em 2. e $\left(f_{\varepsilon}\right)_{\varepsilon}$ é como em 3., então $\left(v_{\varepsilon} \circ u_{\varepsilon}-f_{\varepsilon} \circ w_{\varepsilon}\right)_{\varepsilon} \in \mathcal{E}_{M}\left[\left[t_{0},+\infty\left[; \mathbb{R}^{m}\right]\right.\right.$.

Demonstração: Prova análoga à da Proposição 1.3.14.

A partir da Proposição 1.5.9 temos a seguinte definição.

Definição 1.5.10 Sejam $t_{0} \in \mathbb{R}, v \in \mathcal{G}_{\tau}\left(\mathbb{R}^{n} ; \mathbb{R}^{m}\right)$ e $u \in \mathcal{G}\left(\left[t_{0},+\infty\left[; \mathbb{R}^{n}\right)\right.\right.$. A composta de $u$ e $v$, denotada por $v \circ u$, é o elemento de $\mathcal{G}\left(\left[t_{0},+\infty\left[; \mathbb{R}^{m}\right)\right.\right.$ definido por

$$
v \circ u:=\left(v_{\varepsilon} \circ u_{\varepsilon}\right)_{\varepsilon}+\mathcal{N}\left[\left[t_{0},+\infty\left[; \mathbb{R}^{m}\right],\right.\right.
$$

onde $\left(u_{\varepsilon}\right)_{\varepsilon}$ é um representante qualquer de $u$ e $\left(v_{\varepsilon}\right)_{\varepsilon}$ é um representante qualquer de $v$.

Finalizamos este capítulo "comparando" os conjuntos $\mathcal{E}_{\tau}\left[\left[t_{0},+\infty\left[; \mathbb{R}^{m}\right]\right.\right.$ e $\mathcal{E}_{M}\left[\left[t_{0},+\infty\left[; \mathbb{R}^{m}\right]\right.\right.$ e as álgebras $\mathcal{G}_{\tau}\left(\left[t_{0},+\infty\left[; \mathbb{R}^{m}\right)\right.\right.$ e $\mathcal{G}\left(\left[t_{0},+\infty\left[; \mathbb{R}^{m}\right)\right.\right.$.

Proposição 1.5.11 Seja $t_{0} \in \mathbb{R}$. Então

1. $\mathcal{E}_{\tau}\left[\left[t_{0},+\infty\left[; \mathbb{R}^{m}\right] \subset \mathcal{E}_{M}\left[\left[t_{0},+\infty\left[; \mathbb{R}^{m}\right] ;\right.\right.\right.\right.$

2. $\mathcal{N}_{\tau}\left[\left[t_{0},+\infty\left[; \mathbb{R}^{m}\right] \subset \mathcal{N}\left[\left[t_{0},+\infty\left[; \mathbb{R}^{m}\right]\right.\right.\right.\right.$. 
Demonstração: Decorre das definições de cada conjunto.

A partir da Proposição 1.5 .11 (1.) podemos definir uma aplicação de $\mathcal{G}_{\tau}\left(\left[t_{0},+\infty\left[; \mathbb{R}^{m}\right)\right.\right.$ em $\mathcal{G}\left(\left[t_{0},+\infty\left[; \mathbb{R}^{m}\right)\right.\right.$. Mais precisamente,

$$
\begin{aligned}
\mathcal{G}_{\tau}\left(\left[t_{0},+\infty\left[; \mathbb{R}^{m}\right)\right.\right. & \longrightarrow \mathcal{G}\left(\left[t_{0},+\infty\left[; \mathbb{R}^{m}\right)\right.\right. \\
\left(u_{\varepsilon}\right)_{\varepsilon}+\mathcal{N}_{\tau}\left[\left[t_{0},+\infty\left[; \mathbb{R}^{m}\right]\right.\right. & \longmapsto\left(u_{\varepsilon}\right)_{\varepsilon}+\mathcal{N}\left[\left[t_{0},+\infty\left[; \mathbb{R}^{m}\right]\right.\right.
\end{aligned}
$$

Note que a aplicação acima, como mostra o exemplo a seguir, pode não ser injetora.

Exemplo 1.5.12 Sejam $t_{0} \in \mathbb{R}$ e $\varphi \in \mathscr{C}^{\infty}(\mathbb{R} ; \mathbb{R})$ tal que

$$
\left.\varphi \equiv 1 \text { em }\left[t_{0}-\frac{1}{4}, t_{0}+\frac{1}{4}\right] \text { e } \varphi \equiv 0 \text { em } \mathbb{R} \backslash\right] t_{0}-\frac{1}{2}, t_{0}+\frac{1}{2}[.
$$

$S e\left(v_{\varepsilon}\right)_{\varepsilon}$ é definida por

$$
v_{\varepsilon}(t)=\varphi\left(t+t_{0}-\frac{1}{\varepsilon}\right), \quad \forall(\varepsilon, t) \in \mathbf{I} \times\left[t_{0},+\infty[,\right.
$$

então $\left(v_{\varepsilon}\right)_{\varepsilon} \in \mathcal{E}_{\tau}\left[\left[t_{0},+\infty[; \mathbb{R}],\left(v_{\varepsilon}\right)_{\varepsilon} \in \mathcal{N}\left[\left[t_{0},+\infty[; \mathbb{R}] \operatorname{mas}\left(v_{\varepsilon}\right)_{\varepsilon} \notin \mathcal{N}_{\tau}\left[\left[t_{0},+\infty[; \mathbb{R}]\right.\right.\right.\right.\right.\right.$.

De fato, de $\varphi \in \mathscr{C}^{\infty}(\mathbb{R} ; \mathbb{R})$ ser limitada e $\frac{d^{p} v_{\varepsilon}}{d t^{p}}(t)=\frac{d^{p} \varphi}{d t^{p}}\left(t+t_{0}-\frac{1}{\varepsilon}\right)$, para todo $(\varepsilon, p, t) \in \mathbf{I} \times \mathbb{N} \times\left[t_{0},+\infty\left[\right.\right.$, é claro que $\left(v_{\varepsilon}\right)_{\varepsilon} \in \mathcal{E}_{\tau}\left[\left[t_{0},+\infty[; \mathbb{R}] \quad\right.\right.$ e que $\left(v_{\varepsilon}\right)_{\varepsilon} \in \mathcal{E}_{M}\left[\left[t_{0},+\infty[; \mathbb{R}]\right.\right.$. Para verificar que $\left(v_{\varepsilon}\right)_{\varepsilon} \in \mathcal{N}\left[\left[t_{0},+\infty[; \mathbb{R}]\right.\right.$, considere $p \in \mathbb{N}$, $t_{1}>t_{0}, T>0$ tal que $\left.\left[t_{0}, t_{1}\right] \subset\right]-T, T\left[\right.$ e $\eta:=\frac{1}{T+1}$. Então

$$
\left.A:=\left\{t+t_{0}-\frac{1}{\varepsilon} \mid(\varepsilon, t) \in \mathbf{I}_{\eta} \times\right]-T, T[\} \subset\right]-\infty, t_{0}-1[,
$$

e assim, como $\left.\varphi\right|_{]-\infty, t_{0}-1[} \equiv 0$, temos que $\frac{d^{p} v_{\varepsilon}}{d t^{p}}(t) \equiv 0$ em $]-T, T\left[\right.$. Logo $\frac{d^{p} v_{\varepsilon}}{d t^{p}}(t) \equiv 0$ em $\left[t_{0}, t_{1}\right]$, ou seja $\left(v_{\varepsilon}\right)_{\varepsilon} \in \mathcal{N}\left[\left[t_{0},+\infty[; \mathbb{R}]\right.\right.$. Para finalizar, suponha, por absurdo, que $\left(v_{\varepsilon}\right)_{\varepsilon} \in \mathcal{N}_{\tau}[\mathbb{R} ; \mathbb{R}]$. Então, existe $N \in \mathbb{N}$ tal que, para $r:=N+1$, existem $c>1$ e $\eta_{1} \in \mathbf{I}$ com $\eta_{1}<\frac{1}{2}$ tais que

$$
\left|v_{\varepsilon}(t)\right| \leq c(1+|t|)^{N} \varepsilon^{r}, \quad \forall(\varepsilon, x) \in \mathbf{I}_{\eta_{1}} \times\left[t_{0},+\infty[\right.
$$


isto é,

$$
\left|v_{\varepsilon}(t)\right| \leq c(1+|t|)^{N} \varepsilon^{N+1}, \quad \forall(\varepsilon, x) \in \mathbf{I}_{\eta_{1}} \times\left[t_{0},+\infty[\right.
$$

Portanto,

$$
1=\varphi\left(t_{0}\right)=v_{\varepsilon}\left(\frac{1}{\varepsilon}\right) \leq c\left(1+\frac{1}{\varepsilon}\right)^{N} \varepsilon^{N+1} \leq c\left(\frac{1}{\varepsilon}+\frac{1}{\varepsilon}\right)^{N} \varepsilon^{N+1} \leq 2^{N} c \varepsilon, \quad \forall \varepsilon \in \mathbf{I}_{\eta_{1}},
$$

o que é um absurdo. $\operatorname{Logo}\left(v_{\varepsilon}\right)_{\varepsilon} \notin \mathcal{N}_{\tau}\left[\left[t_{0},+\infty[; \mathbb{R}]\right.\right.$. 


\section{Capítulo 2}

\section{Distribuições e funções generalizadas de}

\section{Colombeau}

O objetivo deste capítulo é apresentar uma imersão do conjunto das distribuições (denotado por $\mathcal{D}^{\prime}(\Omega ; \mathbb{R})$ ) no conjunto das funções generalizadas de Colombeau e uma imersão do conjunto das distribuições temperadas (denotado por $\mathcal{S}^{\prime}\left(\mathbb{R}^{n} ; \mathbb{R}\right)$ ) no conjunto das funções generalizadas temperadas de Colombeau. Essas imersões, que estão nas seções 2.3 e 2.4, respectivamente, foram baseadas nas imersões encontradas em [Del] e [GKOS]. Para facilitar a leitura, introduzimos, na seção 2.1, a definição e algumas propriedades dos conjuntos $\mathcal{D}^{\prime}(\Omega ; \mathbb{R})$ e $\mathcal{S}^{\prime}\left(\mathbb{R}^{n} ; \mathbb{R}\right)$ que nos serão úteis.

\subsection{Os conjuntos $\mathcal{D}^{\prime}(\Omega ; \mathbb{R})$ e $\mathcal{S}^{\prime}\left(\mathbb{R}^{n} ; \mathbb{R}\right)$}

Nesta secão, apresentaremos os conjuntos $\mathcal{D}^{\prime}(\Omega ; \mathbb{R})$ e $\mathcal{S}^{\prime}\left(\mathbb{R}^{n} ; \mathbb{R}\right)$ e destacaremos algumas de suas propriedades que nos serão úteis para obter uma imersão desses conjuntos na teoria das funções generalizadas de Colombeau. Os resultados aqui encontrados foram 
extraídos de [Cor], [CK], [Hör] e [Bar].

Iniciaremos esta seção com um breve estudo sobre o conjunto das funções de suporte compacto que é o domínio de uma distribuição.

Sejam $\Omega$ um aberto de $\mathbb{R}^{n}, \varphi \in \mathscr{C}(\Omega ; \mathbb{R})$. Em todo este trabalho utilizaremos as sequintes notações:

- $\operatorname{supp} \varphi:=\overline{\{x \in \Omega: \varphi(x) \neq 0\}}$

- $\mathscr{C}_{c}(\Omega ; \mathbb{R}):=\{\varphi \in \mathscr{C}(\Omega ; \mathbb{R}) \mid \operatorname{supp} \varphi$ é compacto em $\Omega\}$

- $\mathscr{C}_{c}^{\infty}(\Omega ; \mathbb{R}):=\left\{\varphi \in \mathscr{C}^{\infty}(\Omega ; \mathbb{R}) \mid \operatorname{supp} \varphi\right.$ é compacto em $\left.\Omega\right\}$.

O conjunto supp $\varphi$ é chamado de suporte de $\varphi$. É fácil verificar que $x \notin \operatorname{supp} \varphi$ se, e somente se, existe $\sigma>0$ tal que $\varphi \equiv 0$ em $B_{\sigma}(x)$.

O conjunto $\mathscr{C}_{c}(\Omega ; \mathbb{R})$ é chamado conjunto das funções contínuas de suporte compacto e o conjunto $\mathscr{C}_{c}^{\infty}(\Omega ; \mathbb{R})$ é chamado conjunto das funções infinitamente diferenciáveis de suporte compacto e é também denotado por $\mathcal{D}(\Omega ; \mathbb{R})$.

Notemos que $\mathscr{C}_{c}(\Omega ; \mathbb{R})$ e $\mathscr{C}_{c}^{\infty}(\Omega ; \mathbb{R})$ munidos com as operações usuais de adição de funções e multiplicação de função por número real são $\mathbb{R}$-espaços vetoriais.

Um resultado importante e que, por exemplo, encontra-se em [Cor] é o seguinte:

Se $\Omega$ é um aberto de $\mathbb{R}^{n}$ e $K \subset \subset \Omega$, então existe uma $\varphi \in \mathscr{C}_{c}^{\infty}(\Omega ; \mathbb{R})$ tal que $0 \leq \varphi \leq 1$ e $\varphi \equiv 1$ em um aberto de $\Omega$ contendo $K$.

Além dos conjuntos citados anteriormente, utilizaremos com freqüência a seguinte operação entre funções de $\mathscr{C}\left(\mathbb{R}^{n} ; \mathbb{R}\right)$ e $\mathscr{C}_{c}\left(\mathbb{R}^{n} ; \mathbb{R}\right)$.

Sejam $\varphi \in \mathscr{C}\left(\mathbb{R}^{n} ; \mathbb{R}\right)$ e $\psi \in \mathscr{C}_{c}\left(\mathbb{R}^{n} ; \mathbb{R}\right)$. Denotamos por $\varphi * \psi$, e chamamos de produto de convolução de $\varphi$ e $\psi$, à aplicação que a cada $x \in \mathbb{R}^{n}$ associa o elemento 


$$
(\varphi * \psi)(x):=\int_{R^{n}} \varphi(x-y) \psi(y) d y
$$

Convém destacar que o produto convolução de $\varphi$ e $\psi$ define um elemento de $\mathscr{C}\left(\mathbb{R}^{n} ; \mathbb{R}\right)$ e que,

$$
\begin{gathered}
\operatorname{supp}(\varphi * \psi) \subset \operatorname{supp} \varphi+\operatorname{supp} \psi \\
(\varphi * \psi) * \mu=\varphi *(\psi * \mu), \quad \forall \mu \in \mathscr{C}_{c}\left(\mathbb{R}^{n} ; \mathbb{R}\right) .
\end{gathered}
$$

Além disso, se $\varphi \in \mathscr{C}^{\infty}\left(\mathbb{R}^{n} ; \mathbb{R}\right)$ e $\alpha \in \mathbb{N}^{n}$, então

$$
\begin{gathered}
\varphi * \psi \in \mathscr{C}^{\infty}\left(\mathbb{R}^{n} ; \mathbb{R}\right) ; \\
\partial^{\alpha}(\varphi * \psi)=\left(\partial^{\alpha} \varphi\right) * \psi .
\end{gathered}
$$

Na Definição 2.1.1, apresentaremos um conceito de convergência para famílias de elementos de $\mathscr{C}_{c}^{\infty}(\Omega ; \mathbb{R})$. Conceito esse que será muito útil para apresentar uma caracterização do espaço das distribuições (Teorema 2.1.5).

Definição 2.1.1 Sejam $\Omega$ um aberto de $\mathbb{R}^{n}, \quad\left(\varphi_{\varepsilon}\right)_{\varepsilon \in \mathbf{I}}$ uma família de elementos de $\mathscr{C}_{c}^{\infty}(\Omega ; \mathbb{R})$ e $\varphi \in \mathscr{C}_{c}^{\infty}(\Omega ; \mathbb{R})$. Dizemos que $\left(\varphi_{\varepsilon}\right)_{\varepsilon \in \mathbf{I}}$ converge para $\varphi$ em $\mathscr{C}_{c}^{\infty}(\Omega ; \mathbb{R})$, quando $\varepsilon \downarrow 0$, e escrevemos $\varphi_{\varepsilon} \rightarrow \varphi$, quando $\varepsilon \downarrow 0$, se

(i) $\exists K \subset \subset \Omega$ tal que supp $\varphi_{\varepsilon} \subset K, \quad \forall \varepsilon \in \mathbf{I}$;

(ii) $\forall \alpha \in \mathbb{N}^{n}$, a familia $\left(\partial^{\alpha} \varphi_{\varepsilon}\right)_{\varepsilon \in \mathbf{I}}$ converge uniformente para $\partial^{\alpha} \varphi$, quando $\varepsilon \downarrow 0$.

Após este breve comentário sobre os conjuntos das funções de suporte compacto, estamos em condições de introduzir e apresentar algumas propriedades de uma distribuição sobre $\Omega$. 
Definição 2.1.2 Seja $\Omega$ um aberto de $\mathbb{R}^{n}$. Uma distribuição sobre $\Omega$ é uma função linear $T: \mathscr{C}_{c}^{\infty}(\Omega ; \mathbb{R}) \longrightarrow \mathbb{R}$ com a seguinte propriedade:

$$
\begin{gathered}
\forall K \subset \subset \Omega, \exists c>0 e \exists k \in \mathbb{N} \text { tais que } \\
|T(\varphi)| \leq c \sum_{|\alpha| \leq k}\left\|\partial^{\alpha} \varphi\right\|_{\infty}, \quad \forall \varphi \in \mathscr{C}_{c}^{\infty}(\Omega ; \mathbb{R}) \operatorname{com} \operatorname{supp} \varphi \subseteq K,
\end{gathered}
$$

onde $\left\|\partial^{\alpha} \varphi\right\|_{\infty}:=\sup \left\{\left|\partial^{\alpha} \varphi(x)\right| \mid x \in \Omega\right\}$.

No Exemplo 2.1.3 a seguir, apresentaremos dois exemplos muito importantes de distribuições.

Exemplo 2.1.3 1. Sejam $\Omega$ um aberto de $\mathbb{R}^{n}$ e $a \in \Omega$. Então a função $\delta_{a}: \mathscr{C}_{c}^{\infty}(\Omega ; \mathbb{R}) \longrightarrow \mathbb{R}$ definida por

$$
\delta_{a}(\varphi):=\varphi(a)
$$

é uma distribuição sobre $\Omega$.

2. Sejam $a \in \mathbb{R}$ e $H_{a}: \mathbb{R} \longrightarrow \mathbb{R}$ uma função tal que $H_{a}(x)=0$ se $x<a$, e $H_{a}(x)=1$ se $x>$ a. Então a função $T_{H_{a}}: \mathscr{C}_{c}^{\infty}(\mathbb{R} ; \mathbb{R}) \longrightarrow \mathbb{R}$ definida por

$$
T_{H_{a}}(\varphi):=\int_{R} H_{a}(x) \varphi(x) d x=\int_{[a,+\infty[} \varphi(x) d x
$$

é uma distribuição sobre $\mathbb{R}$.

De fato, para 1. basta observar que

$$
\left|\delta_{a}(\varphi)\right|=|\varphi(a)| \leq\|\varphi\|_{\infty}, \quad \forall \varphi \in \mathscr{C}_{c}^{\infty}(\Omega ; \mathbb{R})
$$

e assim, para todo $K \subset \subset \Omega$ a afirmação (2.5) é verdadeira para $c=1$ e $k=0$. Para 2. basta notar que, se $K_{1} \subset \subset \mathbb{R}$ e $\varphi \in \mathscr{C}_{c}^{\infty}(\mathbb{R} ; \mathbb{R})$ é tal que $\operatorname{supp} \varphi \subseteq K_{1}$, então

$$
\left|\int_{[a,+\infty[} \varphi(x) d x\right| \leq \int_{[a,+\infty[}|\varphi(x)| d x \leq \int_{[-r, r]}|\varphi(x)| d x \leq 2 r\|\varphi\|_{\infty},
$$


onde $r>0$ é tal que $K_{1} \subseteq[-r, r]$. Assim, a afirmação (2.5) é verdadeira para $c=2 r$ e $k=0$.

$O$ elemento $\delta_{a}$ é chamado de Medida de Dirac e, quando $a=0$, escreveremos apenas $\delta$ para denotar $\delta_{0} . A$ distribuição $T_{H_{a}}$ é chamada de Degrau de Heaviside.

O próximo resultado nos fornece uma classe de exemplos muito importantes de distribuições e que, no desenvolvimento deste trabalho, serão muito úteis.

Proposição 2.1.4 Sejam $\Omega$ um aberto de $\mathbb{R}^{n}$ e $f \in \mathscr{C}(\Omega ; \mathbb{R})$. Então a aplicação $d_{f}: \mathscr{C}_{c}^{\infty}(\Omega ; \mathbb{R}) \longrightarrow \mathbb{R}$ definida por

$$
d_{f}(\varphi)=\int_{\Omega} f(x) \varphi(x) d x
$$

é uma distribuição sobre $\Omega$.

Demonstração: Seja $K \subset \subset \Omega$. Como $f \in \mathscr{C}(\Omega ; \mathbb{R})$ e $K \subset \subset \mathbb{R}^{n}$ existe $c_{1}>0$ tal que $|f(x)| \leq c_{1}$, para todo $x \in K$ e existe $r>0$ tal que $K \subset[-r, r]^{n}$. Então, para toda $\varphi \in \mathscr{C}_{c}^{\infty}(\Omega ; \mathbb{R}) \operatorname{com} \operatorname{supp} \varphi \subset K$, temos

$$
\begin{aligned}
\left|d_{f}(\varphi)\right| & =\left|\int_{\Omega} f(x) \varphi(x) d x\right| \\
& =\left|\int_{K} f(x) \varphi(x) d x\right| \\
& \leq \int_{K}|f(x)||\varphi(x)| d x \\
& \leq\|\varphi\|_{\infty} \int_{K}|f(x)| d x \\
& \leq(2 r)^{n} c_{1}\|\varphi\|_{\infty} .
\end{aligned}
$$

Logo, a afirmação (2.5) está satisfeita para $c=(2 r)^{n} c_{1}$ e $k=0$.

Note que, se $\Omega$ é um aberto de $\mathbb{R}^{n}, f \in \mathscr{C}(\Omega ; \mathbb{R})$ e $V$ é um aberto de $\Omega$ podemos 
definir a distribuição

$$
\left.\varphi \in \mathscr{C}_{c}^{\infty}(V ; \mathbb{R}) \longmapsto \int_{V} f\right|_{V}(x) \varphi(x) d x=\int_{V} f(x) \varphi(x) d x,
$$

ou seja a distribuição $d_{f}$, onde $\left.f\right|_{V}$ é a restrição de $f$ a $V$. Para facilitar a escrita, escreveremos $d_{f}$ distribuição sobre $V$ no lugar de $\left.d_{f}\right|_{V}$.

Uma importante caracterização de uma distribuição sobre $\Omega$ é a seguinte.

Teorema 2.1.5 Sejam $\Omega$ um aberto de $\mathbb{R}^{n}$ e $T: \mathscr{C}_{c}^{\infty}(\Omega ; \mathbb{R}) \longrightarrow \mathbb{R}$ uma função linear. São equivalentes:

(a) Té uma distribuição sobre $\Omega$;

(b) para toda $\left(\varphi_{\varepsilon}\right)_{\varepsilon \in \mathbf{I}}$ família de elementos de $\mathscr{C}_{c}^{\infty}(\Omega ; \mathbb{R})$ com $\left(\varphi_{\varepsilon}\right)_{\varepsilon \in \mathbf{I}}$ convergindo para 0 em $\mathscr{C}_{c}^{\infty}(\Omega ; \mathbb{R})$, quando $\varepsilon \downarrow 0$ (Definição 2.1.1), tem-se que $\left(T\left(\varphi_{\varepsilon}\right)\right)_{\varepsilon \in \mathbf{I}}$ converge para 0 , quando $\varepsilon \downarrow 0$, isto é,

$$
\text { se } \varphi_{\varepsilon} \longrightarrow 0, \text { quando } \varepsilon \downarrow 0 \text {, então } \lim _{\varepsilon \downarrow 0} T\left(\varphi_{\varepsilon}\right)=0 \text {. }
$$

Demonstração: Suponhamos $(a)$ verdadeira e seja $\left(\varphi_{\varepsilon}\right)_{\varepsilon \in \mathbf{I}}$ uma família de elementos de $\mathscr{C}_{c}^{\infty}(\Omega ; \mathbb{R})$ tal que $\varphi_{\varepsilon} \rightarrow 0$, quando $\varepsilon \downarrow 0$. Então existe $K \subset \subset \Omega$ tal que supp $\varphi_{\varepsilon} \subset K$, para todo $\varepsilon \in \mathbf{I}$, e $\left(\partial^{\alpha} \varphi_{\varepsilon}\right)_{\varepsilon \in \mathbf{I}}$ converge uniformemente para 0 , quando $\varepsilon \downarrow 0$, para todo $\alpha \in \mathbb{N}^{n}$.

Do fato de $T$ ser uma distribuição sobre $\Omega$, existem $c>0$ e $k \in \mathbb{N}$ tais que (2.5) é verdadeira, e assim,

$$
\left|T\left(\varphi_{\varepsilon}\right)\right| \leq c \sum_{|\alpha| \leq k}\left\|\partial^{\alpha} \varphi_{\varepsilon}\right\|_{\infty}, \quad \forall \varepsilon \in \mathbf{I}
$$

Como $\left(\partial^{\alpha} \varphi_{\varepsilon}\right)_{\varepsilon \in \mathbf{I}}$ converge uniformemente para 0 , quando $\varepsilon \downarrow 0$, para todo $|\alpha| \leq k$, temos que

$$
\lim _{\varepsilon \downarrow 0} \sum_{|\alpha| \leq k}\left\|\partial^{\alpha} \varphi_{\varepsilon}\right\|_{\infty}=\sum_{|\alpha| \leq k} \lim _{\varepsilon \downarrow 0}\left\|\partial^{\alpha} \varphi_{\varepsilon}\right\|_{\infty}=0
$$


Logo, por (2.7), temos que $\lim _{\varepsilon \downarrow 0} T\left(\varphi_{\varepsilon}\right)=0$, o que prova $(b)$.

Provaremos, a seguir, que (b) implica (a). Para isso, suponhamos que $T$ não seja uma distribuição sobre $\Omega$. Então existe $K \subset \subset \Omega$ tal que, para todo $j \in \mathbb{N}^{*}$, existe $\varphi_{j} \in \mathscr{C}_{c}^{\infty}(\Omega ; \mathbb{R}) \mathrm{com}$

$$
\operatorname{supp} \varphi_{j} \subset K \quad \text { e }\left|T\left(\varphi_{j}\right)\right|>j \sum_{|\beta| \leq j}\left\|\partial^{\beta} \varphi_{j}\right\|_{\infty} .
$$

Considere a família $\left(\psi_{\varepsilon}\right)_{\varepsilon \in \mathbf{I}}$ de elementos de $\mathscr{C}_{c}^{\infty}(\Omega ; \mathbb{R})$ definida por

$$
\psi_{\varepsilon}:=\frac{\varphi_{k+1}}{\left|T\left(\varphi_{k+1}\right)\right|} \quad \text { se } \quad \frac{1}{k+1}<\varepsilon \leq \frac{1}{k} .
$$

Provaremos que $\psi_{\varepsilon} \rightarrow 0$, quando $\varepsilon \downarrow 0$.

É claro que supp $\psi_{\varepsilon} \subset K$, para todo $\varepsilon \in \mathbf{I}$.

Seja $\alpha \in \mathbb{N}^{n}$ e considere $j_{0} \in \mathbb{N}$ com $j_{0}>|\alpha|$. Então, para $\varepsilon \leq \frac{1}{j_{0}}$, temos que $\frac{1}{k+1}<\varepsilon \leq \frac{1}{k}$ para algum $k \geq j_{0}$. Logo, por (2.8), segue que

$$
\begin{aligned}
\left\|\partial^{\alpha} \psi_{\varepsilon}\right\|_{\infty} & =\frac{1}{\left|T\left(\varphi_{k+1}\right)\right|}\left\|\partial^{\alpha} \varphi_{k+1}\right\|_{\infty} \\
& \leq \frac{1}{\left|T\left(\varphi_{k+1}\right)\right|} \sum_{|\beta| \leq|\alpha|}\left\|\partial^{\beta} \varphi_{k+1}\right\|_{\infty} \\
& \leq \frac{1}{\left|T\left(\varphi_{k+1}\right)\right|} \sum_{|\beta| \leq k+1}\left\|\partial^{\beta} \varphi_{k+1}\right\|_{\infty} \\
& \leq \frac{1}{k+1} \\
& \leq \varepsilon .
\end{aligned}
$$

Portanto

$$
\left.\left.\left\|\partial^{\alpha} \psi_{\varepsilon}\right\|_{\infty}<\varepsilon, \quad \forall \varepsilon \in\right] 0, \frac{1}{j_{0}}\right]
$$

ou seja, $\left(\partial^{\alpha} \psi_{\varepsilon}\right)_{\varepsilon \in \mathbf{I}}$ converge uniformemente para 0 , quando $\varepsilon \downarrow 0$.

De $\alpha \in \mathbb{N}^{n}$ ser arbitrário concluímos que $\psi_{\varepsilon} \rightarrow 0$, quando $\varepsilon \downarrow 0$, o que, juntamente com o fato de $\left|T\left(\psi_{\varepsilon}\right)\right|=1$ para todo $\varepsilon \in \mathbf{I}$, contradiz a afirmação $(b)$.

O Teorema 2.1 .5 , observando que $\mathcal{D}(\Omega ; \mathbb{R})$ também denota o conjunto $\mathscr{C}_{c}^{\infty}(\Omega ; \mathbb{R})$, justifica a seguinte notação. 
Notação 2.1.6 Seja $\Omega$ um aberto de $\mathbb{R}^{n}$. Denotamos por $\mathcal{D}^{\prime}(\Omega ; \mathbb{R})$ o conjunto das distribuições sobre $\Omega$.

Convém observar que $\mathcal{D}^{\prime}(\Omega ; \mathbb{R})$ é um subespaço vetorial do espaço vetorial $\mathbb{R}^{\mathscr{C}_{c}^{\infty}(\Omega ; R)}$. Além disso a aplicação

$$
\begin{aligned}
\mathscr{C}(\Omega ; \mathbb{R}) & \longrightarrow \mathcal{D}^{\prime}(\Omega ; \mathbb{R}) \\
f & \longmapsto d_{f}
\end{aligned}
$$

onde $d_{f}$ é a distribuição sobre $\Omega$ definida em (2.6), é um homomorfismo injetor de álgebras [Cor]. Por esse motivo, $\mathscr{C}(\Omega ; \mathbb{R})$ pode ser considerado como um subespaço de $\mathcal{D}^{\prime}(\Omega ; \mathbb{R})$.

A Observação 2.1.7 será útil para ter sentido a Definição 2.1.8.

Observação 2.1.7 Sejam $\Omega$ um aberto de $\mathbb{R}^{n}, T \in \mathcal{D}^{\prime}(\Omega ; \mathbb{R}), f \in \mathscr{C}^{\infty}(\Omega ; \mathbb{R})$ e $\alpha \in \mathbb{N}^{n}$. Então as aplicações lineares dadas por

$$
\begin{gathered}
\varphi \in \mathscr{C}_{c}^{\infty}(\Omega ; \mathbb{R}) \longmapsto T(f \varphi) ; \\
\varphi \in \mathscr{C}_{c}^{\infty}(\Omega ; \mathbb{R}) \longmapsto(-1)^{|\alpha|} T\left(\partial^{\alpha} \varphi\right)
\end{gathered}
$$

são distribuições sobre $\Omega$.

De fato, basta usar o Teorema 2.1 .5 e observar que, se $\left(\varphi_{\varepsilon}\right)_{\varepsilon \in \mathbf{I}}$ é uma família de elementos de $\mathscr{C}_{c}^{\infty}(\Omega ; \mathbb{R})$ tal que $\varphi_{\varepsilon} \rightarrow 0$, quando $\varepsilon \downarrow 0$, então $\left(f \varphi_{\varepsilon}\right)_{\varepsilon \in \mathbf{I}}$ e $\left(\partial^{\alpha} \varphi_{\varepsilon}\right)_{\varepsilon \in \mathbf{I}}$ são famílias de elementos de $\mathscr{C}_{c}^{\infty}(\Omega ; \mathbb{R})$ tais que $f \varphi_{\varepsilon} \rightarrow 0$ e $\partial^{\alpha} \varphi_{\varepsilon} \rightarrow 0$, quando $\varepsilon \downarrow 0$.

Definição 2.1.8 Sejam $\Omega$ um aberto de $\mathbb{R}^{n}, T \in \mathcal{D}^{\prime}(\Omega ; \mathbb{R}), f \in \mathscr{C}^{\infty}(\Omega ; \mathbb{R})$ e $\alpha \in \mathbb{N}^{n}$.

1. A distribuição sobre $\Omega$ definida por

$$
\varphi \in \mathscr{C}_{c}^{\infty}(\Omega ; \mathbb{R}) \longmapsto T(f \varphi)
$$

é chamada de produto de f por $T$ e é denotada por $f T$. 
2. A distribuição sobre $\Omega$ definida por

$$
\varphi \in \mathscr{C}_{c}^{\infty}(\Omega ; \mathbb{R}) \longmapsto(-1)^{|\alpha|} T\left(\partial^{\alpha} \varphi\right)
$$

é chamada de derivada parcial de ordem $|\alpha|$ de $T$ e é denotada por $\partial^{\alpha} T$.

Uma função que nos será muito útil é a obtida pelo produto de convolução entre uma distribuição sobre $\mathbb{R}^{n}$ e um elemento de $\mathscr{C}_{c}^{\infty}\left(\mathbb{R}^{n} ; \mathbb{R}\right)$, isto é,

Definição 2.1.9 Sejam $T \in \mathcal{D}^{\prime}\left(\mathbb{R}^{n} ; \mathbb{R}\right)$ e $\varphi \in \mathscr{C}_{c}^{\infty}\left(\mathbb{R}^{n} ; \mathbb{R}\right)$. A aplicação dada por:

$$
x \in \mathbb{R}^{n} \longmapsto T(\varphi(x-\bullet))
$$

é chamada de produto de convolução entre $T$ e $\varphi$, e é denotada por $T * \varphi$.

Note que, se $f \in \mathscr{C}\left(\mathbb{R}^{n} ; \mathbb{R}\right)$ e $\varphi \in \mathscr{C}_{c}^{\infty}\left(\mathbb{R}^{n} ; \mathbb{R}\right)$, então

$$
(f * \varphi)(x)=\int_{R^{n}} f(y) \varphi(x-y) d y=d_{f}(\varphi(x-\bullet))=\left(d_{f} * \varphi\right)(x),
$$

onde $d_{f}$ é a distribuição sobre $\mathbb{R}^{n}$ definida em (2.6) substituindo $\Omega$ por $\mathbb{R}^{n}$.

Destacamos as seguintes propriedades do produto de convolução cujas provas podem ser encontradas, por exemplo, em [Hör].

$\operatorname{Sejam} \varphi, \psi \in \mathscr{C}_{c}^{\infty}\left(\mathbb{R}^{n} ; \mathbb{R}\right), T \in \mathcal{D}^{\prime}\left(\mathbb{R}^{n} ; \mathbb{R}\right)$ e $\alpha \in \mathbb{N}^{n}$. Então

$$
\begin{gathered}
T * \varphi \in \mathscr{C}^{\infty}\left(\mathbb{R}^{n} ; \mathbb{R}\right) ; \\
(T * \varphi) * \psi=T *(\varphi * \psi) ; \\
\partial^{\alpha}(T * \varphi)=T *\left(\partial^{\alpha} \varphi\right) .
\end{gathered}
$$

No espaço das distribuições temos o seguinte conceito de convergência. 
Definição 2.1.10 Sejam $\Omega$ um aberto de $\mathbb{R}^{n}, \quad\left(T_{\varepsilon}\right)_{\varepsilon \in \mathbf{I}}$ uma família de elementos de $\mathcal{D}^{\prime}(\Omega ; \mathbb{R})$ e $T \in \mathcal{D}^{\prime}(\Omega ; \mathbb{R})$. Dizemos que $\left(T_{\varepsilon}\right)_{\varepsilon \in \mathbf{I}}$ converge para $T$ em $\mathcal{D}^{\prime}(\Omega ; \mathbb{R})$, e escrevemos $T_{\varepsilon} \rightarrow T$, quando $\varepsilon \downarrow 0$, se, e somente se, $\left(T_{\varepsilon}(\varphi)\right)_{\varepsilon \in \mathbf{I}}$ converge para $T(\varphi)$, quando $\varepsilon \downarrow 0$, para toda $\varphi \in \mathscr{C}_{c}^{\infty}(\Omega ; \mathbb{R})$.

Exemplo 2.1.11 Seja $\mu \in \mathscr{C}_{c}^{\infty}\left(\mathbb{R}^{n} ; \mathbb{R}\right)$ tal que $\int_{R^{n}} \mu(x) d x=1$. Para todo $\varepsilon \in \mathbf{I}$, seja $\mu_{\varepsilon} a$ função definida em $\mathbb{R}^{n}$ por

$$
\mu_{\varepsilon}(x):=\frac{1}{\varepsilon^{n}} \mu\left(\frac{x}{\varepsilon}\right) .
$$

Então, para todo $\varepsilon \in \mathbf{I}$, tem-se $\mu_{\varepsilon} \in \mathscr{C}_{c}^{\infty}\left(\mathbb{R}^{n} ; \mathbb{R}\right)$ e supp $\mu_{\varepsilon}=\varepsilon$ supp $\mu$. Além disso, $d_{\mu_{\varepsilon}} \rightarrow \delta$ em $\mathcal{D}^{\prime}\left(\mathbb{R}^{n} ; \mathbb{R}\right)$, quando $\varepsilon \downarrow 0$, onde $\delta$ é a Medida de Dirac (Exemplo 2.1 .3 (1.)) e $d_{\mu_{\varepsilon}}$ é a distribuição sobre $\mathbb{R}^{n}$ definida em (2.6) substituindo $\Omega$ por $\mathbb{R}^{n}$.

De fato, é claro que $\mu_{\varepsilon} \in \mathscr{C}_{c}^{\infty}\left(\mathbb{R}^{n} ; \mathbb{R}\right)$ e supp $\mu_{\varepsilon}=\varepsilon \operatorname{supp} \mu$, para todo $\varepsilon \in \mathbf{I}$. Sejam $\varphi \in \mathscr{C}_{c}^{\infty}\left(\mathbb{R}^{n} ; \mathbb{R}\right)$ e $r>0$ tal que supp $\mu \subset[-r, r]^{n}$. Então

$$
\begin{aligned}
d_{\mu_{\varepsilon}}(\varphi)-\delta(\varphi) & =\int_{R^{n}} \mu_{\varepsilon}(x) \varphi(x) d x-\varphi(0) \\
& =\int_{R^{n}} \mu\left(\frac{x}{\varepsilon}\right) \varphi(x) \frac{1}{\varepsilon^{n}} d x-\int_{R^{n}} \varphi(0) \mu(x) d x \\
& =\int_{R^{n}} \mu(x)[\varphi(\varepsilon x)-\varphi(0)] d x \\
& =\int_{[-r, r]^{n}} \mu(x)[\varphi(\varepsilon x)-\varphi(0)] d x,
\end{aligned}
$$

para todo $\varepsilon \in \mathbf{I}$. Note que, pelo Teorema do Valor Médio, temos que

$$
|\varphi(\varepsilon x)-\varphi(0)| \leq\left(\sum_{|\alpha|=1}\left\|\partial^{\alpha} \varphi\right\|_{\infty}\right)|\varepsilon x| \leq 2 n r\left(\sum_{|\alpha|=1}\left\|\partial^{\alpha} \varphi\right\|_{\infty}\right) \varepsilon
$$

para todo $(\varepsilon, x) \in \mathbf{I} \times[-r, r]^{n}$. Assim,

$$
\begin{aligned}
\left|d_{\mu_{\varepsilon}}(\varphi)-\varphi(0)\right| & \leq \int_{[-r, r]^{n}}|\mu(x)||\varphi(\varepsilon x)-\varphi(0)| d x \\
& \leq\left[2 n r\left(\sum_{|\alpha|=1}\left\|\partial^{\alpha} \varphi\right\|_{\infty}\right) \int_{R^{n}}|\mu(x)| d x\right] \varepsilon
\end{aligned}
$$


para todo $\varepsilon \in \mathbf{I}$. Logo, $\lim _{\varepsilon \downarrow 0} d_{\mu_{\varepsilon}}(\varphi)=\varphi(0)$.

Apresentamos, a seguir, a definição de restrição de uma distribuição.

Definição 2.1.12 Seja $\Omega$ um aberto de $\mathbb{R}^{n}, W \subset \Omega$ um aberto de $\mathbb{R}^{n}$ e $T \in \mathcal{D}^{\prime}(\Omega ; \mathbb{R})$. A restrição de $T$ a W, denotada por $\left.T\right|_{W}$ é a aplicaçãa definida por

$$
\left.T\right|_{W}(\varphi):=T(\varphi), \quad \forall \varphi \in \mathscr{C}_{c}^{\infty}(\Omega ; \mathbb{R}) \text { com supp } \varphi \subset W .
$$

Finalizamos este breve estudo do conjunto $\mathcal{D}^{\prime}(\Omega ; \mathbb{R})$ destacando o seguinte resultado que será fundamental na seção 2 deste capítulo e cuja prova pode ser encontrada, por exemplo, em [Bar].

Sejam $\Omega$ e $\Omega^{\prime}$ abertos de $\mathbb{R}^{n}$ e $T \in \mathcal{D}^{\prime}(\Omega ; \mathbb{R})$. Se $\overline{\Omega^{\prime}} \subset \subset \Omega$, então existem $\alpha \in \mathbb{N}^{n}$, $f \in \mathscr{C}_{c}\left(\mathbb{R}^{n} ; \mathbb{R}\right)$ e $W$ aberto de $\mathbb{R}^{n}$ tais que

$$
\overline{\Omega^{\prime}} \subset W \subset \Omega, \quad \operatorname{supp} f \subset W \quad \text { e }\left.T\right|_{\Omega^{\prime}}=\partial^{\alpha} d_{f}
$$

onde $d_{f}$ é a distribuição definida em (2.6).

Antes de apresentar algumas propriedades do conjunto $\mathcal{S}^{\prime}\left(\mathbb{R}^{n} ; \mathbb{R}\right)$ lembramos que $\mathcal{S}\left(\mathbb{R}^{n} ; \mathbb{R}\right)$ é o conjunto das funções infinitamente diferenciáveis que são rapidamente decrescentes no infinito. Mais precisamente,

\section{Definição 2.1.13 Definimos}

$$
\begin{gathered}
\mathcal{S}\left(\mathbb{R}^{n} ; \mathbb{R}\right):=\left\{f \in \mathscr{C}^{\infty}\left(\mathbb{R}^{n} ; \mathbb{R}\right) \mid \forall \alpha \in \mathbb{N}^{n}, \quad \forall m \in \mathbb{N}, \exists c>0\right. \text { tal que } \\
\left.\left|\partial^{\alpha} f(x)\right| \leq c(1+|x|)^{-m}, \quad \forall x \in \mathbb{R}^{n}\right\} .
\end{gathered}
$$

Convém observar o seguinte: 
Observação 2.1.14 1. $\mathcal{S}\left(\mathbb{R}^{n} ; \mathbb{R}\right)$ é um espaço vetorial.

2. Se $f \in \mathcal{S}\left(\mathbb{R}^{n} ; \mathbb{R}\right)$ e g é um polinômio, então $g f \in \mathcal{S}\left(\mathbb{R}^{n} ; \mathbb{R}\right)$.

3. Se $f \in \mathcal{S}\left(\mathbb{R}^{n} ; \mathbb{R}\right)$, então $f$ é limitada $e \int_{R^{n}}|x|^{s}|f(x)| d x<+\infty$, para todo $s \in \mathbb{N}$.

4. Se $f \in \mathcal{S}\left(\mathbb{R}^{n} ; \mathbb{R}\right)$, então $f$ é uniformemente contínua em $\mathbb{R}^{n}$.

De fato, as três primeiras asserções são imediatas. Para verificarmos a afirmação 4., seja $b>0$. De $f \in \mathcal{S}\left(\mathbb{R}^{n} ; \mathbb{R}\right)$ existe $c>0$ tal que

$$
|f(x)| \leq \frac{c}{1+|x|}, \quad \forall x \in \mathbb{R}^{n}
$$

Considere $r=\frac{4 c}{b}$. Como $\left.f\right|_{\frac{B_{r}(0)}{}}$ é uniformemente contínua, existe $s_{1}>0$ tal que

$$
\text { se } \quad x, y \in \overline{B_{r}(0)} \quad \text { e } \quad|x-y|<s_{1}, \quad \text { então } \quad|f(x)-f(y)|<\frac{b}{2 c+1} \text {. }
$$

Note que, se $s_{2}:=\min \left\{s_{1}, \frac{r}{4}\right\}$ e se $x, y \in \mathbb{R}^{n}$ são tais que $|x-y|<s_{2}$, então $x, y \in \overline{B_{r}(0)}$ ou $x, y \in \mathbb{R}^{n} \backslash \overline{B_{r / 2}(0)}$. Dessa forma, se $|x-y|<s_{2}$ temos, por (2.14) e (2.15), que

$$
\begin{gathered}
|f(x)-f(y)|<\frac{b}{2 c+1}<b, \text { se } x, y \in \overline{B_{r}(0)} \\
|f(x)-f(y)|<c\left(\frac{1}{1+|x|}+\frac{1}{1+|y|}\right)<\frac{4 c}{r}=b, \text { se } x, y \in \mathbb{R}^{n} \backslash \overline{B_{r / 2}(0)} .
\end{gathered}
$$

Logo, $f$ é uniformemente contínua em $\mathbb{R}^{n}$.

No espaço vetorial $\mathcal{S}\left(\mathbb{R}^{n} ; \mathbb{R}\right)$ vamos considerar o seguinte conceito de convergência.

Definição 2.1.15 Seja $\left(\varphi_{\varepsilon}\right)_{\varepsilon \in \mathbf{I}}$ uma família de elementos de $\mathcal{S}\left(\mathbb{R}^{n} ; \mathbb{R}\right)$. Dizemos que $\left(\varphi_{\varepsilon}\right)_{\varepsilon \in \mathbf{I}}$ converge para a função nula em $\mathcal{S}\left(\mathbb{R}^{n} ; \mathbb{R}\right)$, quando $\varepsilon \downarrow 0$, e escrevemos $\varphi_{\varepsilon} \longrightarrow 0$, quando $\varepsilon \downarrow 0$, se para todo $(\alpha, \beta) \in \mathbb{N}^{n} \times \mathbb{N}^{n}$ tem-se que a família $\left(\psi_{\alpha, \beta, \varepsilon}\right)_{\varepsilon \in \mathbf{I}}$, onde $\psi_{\alpha, \beta, \varepsilon}$ é a função definida em $\mathbb{R}^{n}$ por $\psi_{\alpha, \beta, \varepsilon}(x)=x^{\beta} \partial^{\alpha} \varphi_{\varepsilon}(x)$, converge uniformemente para 0 , quando $\varepsilon \downarrow 0$. 
Utilizando o conceito de convergência em $\mathcal{S}\left(\mathbb{R}^{n} ; \mathbb{R}\right)$ tem-se a seguinte definição.

Definição 2.1.16 Uma distribuição temperada é uma função linear $T: \mathcal{S}\left(\mathbb{R}^{n} ; \mathbb{R}\right) \longrightarrow \mathbb{R}$ com a seguinte propriedade:

para toda $\left(\varphi_{\varepsilon}\right)_{\varepsilon \in \mathbf{I}}$ familia de elementos de $\mathcal{S}\left(\mathbb{R}^{n} ; \mathbb{R}\right)$ com $\left(\varphi_{\varepsilon}\right)_{\varepsilon \in \mathbf{I}}$ convergindo para a função nula em $\mathcal{S}\left(\mathbb{R}^{n} ; \mathbb{R}\right)$, quando $\varepsilon \downarrow 0$, tem-se que a família $\left(T\left(\varphi_{\varepsilon}\right)\right)_{\varepsilon \in \mathbf{I}}$ converge para 0 , quando $\varepsilon \downarrow 0$, isto é,

$$
\text { se } \varphi_{\varepsilon} \longrightarrow 0, \quad \text { então } \lim _{\varepsilon \downarrow 0} T\left(\varphi_{\varepsilon}\right)=0
$$

A Definição 2.1.16 justifica a seguinte notação.

Notação 2.1.17 Denotamos por $\mathcal{S}^{\prime}\left(\mathbb{R}^{n} ; \mathbb{R}\right)$ o conjunto das distribuições temperadas.

No espaço das distribuições temperadas temos o seguinte conceito de convergência.

Definição 2.1.18 Sejam $T \in \mathcal{S}^{\prime}\left(\mathbb{R}^{n} ; \mathbb{R}\right) \quad e \quad\left(T_{\varepsilon}\right)_{\varepsilon \in \mathbf{I}}$ uma família de elementos de $\mathcal{S}^{\prime}\left(\mathbb{R}^{n} ; \mathbb{R}\right)$. Dizemos que $\left(T_{\varepsilon}\right)_{\varepsilon \in \mathbf{I}}$ converge para $T$ em $\mathcal{S}^{\prime}\left(\mathbb{R}^{n} ; \mathbb{R}\right)$, quando $\varepsilon \downarrow 0$, e escrevemos $T_{\varepsilon} \rightarrow T$, quando $\varepsilon \downarrow 0$, se e somente se, $\left(T_{\varepsilon}(\varphi)\right)_{\varepsilon \in \mathbf{I}}$ converge para $T(\varphi)$, quando $\varepsilon \downarrow 0$, para toda $\varphi \in \mathcal{S}\left(\mathbb{R}^{n} ; \mathbb{R}\right)$.

Note que, da definição de $\mathcal{S}\left(\mathbb{R}^{n} ; \mathbb{R}\right)$, tem-se

$$
\mathscr{C}_{c}^{\infty}\left(\mathbb{R}^{n} ; \mathbb{R}\right) \subset \mathcal{S}\left(\mathbb{R}^{n} ; \mathbb{R}\right) \subset \mathscr{C}^{\infty}\left(\mathbb{R}^{n} ; \mathbb{R}\right)
$$

Desse fato, e observando que, se $\varphi_{\varepsilon} \rightarrow 0$ em $\mathscr{C}_{c}^{\infty}\left(\mathbb{R}^{n} ; \mathbb{R}\right)$, quando $\varepsilon \downarrow 0$, tem-se $\varphi_{\varepsilon} \rightarrow 0$ em $\mathcal{S}\left(\mathbb{R}^{n} ; \mathbb{R}\right)$, quando $\varepsilon \downarrow 0$, concluímos que $\left.T\right|_{\mathscr{C}_{c}^{\infty}\left(R^{n} ; R\right)} \in \mathcal{D}^{\prime}\left(\mathbb{R}^{n} ; \mathbb{R}\right)$, para toda 
$T \in \mathcal{S}^{\prime}\left(\mathbb{R}^{n} ; \mathbb{R}\right)$ (Teorema 2.1.5). Assim podemos definir a aplicação linear

$$
\begin{aligned}
\Phi: \mathcal{S}^{\prime}\left(\mathbb{R}^{n} ; \mathbb{R}\right) & \longrightarrow \mathcal{D}^{\prime}\left(\mathbb{R}^{n} ; \mathbb{R}\right) \\
T & \left.\longmapsto T\right|_{\mathscr{C}_{c}^{\infty}\left(R^{n} ; R\right)}
\end{aligned}
$$

É claro que $\Phi$ é contínua. Além disso, do fato de $\mathscr{C}_{c}^{\infty}\left(\mathbb{R}^{n} ; \mathbb{R}\right)$ ser denso em $\mathcal{S}\left(\mathbb{R}^{n} ; \mathbb{R}\right)$ ([Bar]), temos que $\Phi$ é injetora.

Exemplo 2.1.19 Seja $a \in \mathbb{R}^{n}$. A aplicação $\widetilde{\delta}_{a}: \mathcal{S}\left(\mathbb{R}^{n} ; \mathbb{R}\right) \longrightarrow \mathbb{R}$ definida por

$$
\widetilde{\delta}_{a}(\varphi)=\varphi(a)
$$

é uma distribuição temperada.

De fato, basta observar que, se $\varphi_{\varepsilon} \longrightarrow 0$, quando $\varepsilon \downarrow 0$, então $\left(\varphi_{\varepsilon}\right)_{\varepsilon \in \mathbf{I}}$ converge uniformemente para 0 , quando $\varepsilon \downarrow 0$, e assim, $\left(\varphi_{\varepsilon}(a)\right)_{\varepsilon \in \mathbf{I}}$ converge para 0 , quando $\varepsilon \downarrow 0$.

Note que $\left.\widetilde{\delta}_{a}\right|_{\mathscr{C}_{c}^{\infty}\left(R^{n} ; R\right)}=\delta_{a}$, onde $\delta_{a}$ é a Medida de Dirac (Exemplo 2.1.3 (1.)). Por esse motivo escreveremos $\delta_{a}$ distribuição temperada em vez de $\widetilde{\delta}_{a}$.

Para facilitar a escrita de alguns resultados, introduziremos a seguinte definição.

Definição 2.1.20 Dizemos que $f$ tem crescimento lento no infinito, e escrevemos $f$ tem CLI, se $f$ tem a seguinte propriedade:

$$
\exists N \in \mathbb{N} \text { e } \exists c>0 \text { tais que }|f(x)| \leq c(1+|x|)^{N}, \quad \forall x \in \mathbb{R}^{n} .
$$

Observe que, se $f \in \mathcal{O}_{M}\left(\mathbb{R}^{n} ; \mathbb{R}\right)$ (Definição 1.3.16) ou $f \in \mathscr{C}_{c}\left(\mathbb{R}^{n} ; \mathbb{R}\right)$, então $f$ tem CLI. 
A Proposição 2.1.21 nos fornecerá mais um exemplo importante de distribuição temperada.

Proposição 2.1.21 Seja $f \in \mathscr{C}\left(\mathbb{R}^{n} ; \mathbb{R}\right)$ tal que $f$ tem CLI. Então a aplicação $\widetilde{d}_{f}: \mathcal{S}\left(\mathbb{R}^{n} ; \mathbb{R}\right) \longrightarrow \mathbb{R}$ definida por

$$
\widetilde{d}_{f}(\varphi)=\int_{R^{n}} f(x) \varphi(x) d x
$$

é uma distribuição temperada.

Demonstração: Como $f$ tem $C L I$, existem $N \in \mathbb{N}$ e $c>0$ tais que

$$
|f(x)| \leq c(1+|x|)^{N}, \quad \forall x \in \mathbb{R}^{n} .
$$

Seja $\left(\varphi_{\varepsilon}\right)_{\varepsilon}$ uma família de elementos de $\mathcal{S}\left(\mathbb{R}^{n} ; \mathbb{R}\right)$ tal que $\varphi_{\varepsilon} \rightarrow 0$, quando $\varepsilon \downarrow 0$.

Note que

$$
\begin{aligned}
\left|\widetilde{d}_{f}\left(\varphi_{\varepsilon}\right)\right| & =\left|\int_{R^{n}} f(x) \varphi_{\varepsilon}(x) d x\right| \\
& \leq \int_{R^{n}}|f(x)|\left|\varphi_{\varepsilon}(x)\right| d x \\
& \leq \int_{R^{n}} c(1+|x|)^{N}\left|\varphi_{\varepsilon}(x)\right| d x
\end{aligned}
$$

e assim

$$
\left|\widetilde{d}_{f}\left(\varphi_{\varepsilon}\right)\right| \leq \int_{R^{n}} \frac{c}{(1+|x|)^{2 n}}(1+|x|)^{N+2 n}\left|\varphi_{\varepsilon}(x)\right| d x, \quad \forall \varepsilon \in \mathbf{I} .
$$

Sejam $g: \mathbb{R}^{n} \longrightarrow \mathbb{R}$ e $\left(g_{\varepsilon}\right)_{\varepsilon}$ a família de funções definidas por

$$
\begin{gathered}
g(x):=\frac{c}{(1+|x|)^{2 n}}, \quad \forall x \in \mathbb{R}^{n} ; \\
g_{\varepsilon}(x):=(1+|x|)^{N+2 n}\left|\varphi_{\varepsilon}(x)\right|, \quad \forall x \in \mathbb{R}^{n} .
\end{gathered}
$$

Observe que $g$ é integrável em $\mathbb{R}^{n}$ e que

$$
\begin{aligned}
\left|g_{\varepsilon}(x)\right| & \leq\left(1+\sum_{|\beta|=1}\left|x^{\beta}\right|\right)^{N+2 n}\left|\varphi_{\varepsilon}(x)\right| \\
& \leq\left(2 \max \left\{1, n \max \left\{\left|x^{\beta}\right|:|\beta|=1\right\}\right\}\right)^{N+2 n}\left|\varphi_{\varepsilon}(x)\right| \\
& \leq 2^{N+2 n}\left|\varphi_{\varepsilon}(x)\right|+2^{N+2 n} n^{N+2 n} \sum_{|\gamma|=N+2 n}\left|x^{\gamma} \varphi_{\varepsilon}(x)\right|
\end{aligned}
$$


para todo $(\varepsilon, x) \in \mathbf{I} \times \mathbb{R}^{n}$. Logo, como $\varphi_{\varepsilon} \longrightarrow 0$, temos que $\left(\left\|g_{\varepsilon}\right\|_{\infty}\right)_{\varepsilon}$ converge para 0 , ou seja, dado $b>0$ existe $\eta_{b} \in \mathbf{I}$ tal que

$$
\left|g_{\varepsilon}(x)\right| \leq \frac{b}{\int_{R^{n}} g(x) d x}, \quad \forall(\varepsilon, x) \in \mathbf{I}_{\eta_{b}} \times \mathbb{R}^{n}
$$

De (2.17) e (2.18) temos que

$$
\left|\tilde{d}_{f}\left(\varphi_{\varepsilon}\right)\right| \leq b, \quad \forall(\varepsilon, x) \in \mathbf{I}_{\eta_{b}} \times \mathbb{R}^{n}
$$

Portanto, como $\delta>0$ é arbitrário, concluímos que

$$
\lim _{\varepsilon \downarrow 0} \widetilde{d}_{f}\left(\varphi_{\varepsilon}\right)=0
$$

e assim, $\widetilde{d}_{f}$ é uma distribuição temperada.

Tendo em vista que $\left.\tilde{d}_{f}\right|_{\mathscr{C}_{c}^{\infty}\left(R^{n} ; R\right)}=d_{f}$, onde $d_{f}$ é a distribuição sobre $\mathbb{R}^{n}$ definida em (2.6) substituindo $\Omega$ por $\mathbb{R}^{n}$, escreveremos $d_{f}$ distribuição temperada em vez de $\widetilde{d}_{f}$.

A Observação 2.1.22 será útil para apresentarmos a próxima definição.

Observação 2.1.22 Seja $T \in \mathcal{S}^{\prime}\left(\mathbb{R}^{n} ; \mathbb{R}\right), \quad f \in \mathcal{O}_{M}\left(\mathbb{R}^{n} ; \mathbb{R}\right)$ e $\alpha \in \mathbb{N}^{n}$. Então as aplicações lineares dadas por

$$
\begin{gathered}
\varphi \in \mathcal{S}\left(\mathbb{R}^{n} ; \mathbb{R}\right) \longmapsto T(f \varphi) ; \\
\varphi \in \mathcal{S}\left(\mathbb{R}^{n} ; \mathbb{R}\right) \longmapsto(-1)^{|\alpha|} T\left(\partial^{\alpha} \varphi\right)
\end{gathered}
$$

são distribuições temperadas.

De fato, basta observar que, se $\left(\varphi_{\varepsilon}\right)_{\varepsilon \in \mathbf{I}}$ é uma família de elementos de $\mathcal{S}\left(\mathbb{R}^{n} ; \mathbb{R}\right)$ tal que $\varphi_{\varepsilon} \longrightarrow 0$, quando $\varepsilon \downarrow 0$, então $\left(f \varphi_{\varepsilon}\right)_{\varepsilon \in \mathbf{I}}$ e $\left(\partial^{\alpha} \varphi_{\varepsilon}\right)_{\varepsilon \in \mathbf{I}}$ são famílias de elementos de $\mathcal{S}\left(\mathbb{R}^{n} ; \mathbb{R}\right)$ tais que $f \varphi_{\varepsilon} \longrightarrow 0$ e $\partial^{\alpha} \varphi_{\varepsilon} \rightarrow 0$, quando $\varepsilon \downarrow 0$. 
Definição 2.1.23 Sejam $\Omega$ um aberto de $\mathbb{R}^{n}, T \in \mathcal{S}^{\prime}\left(\mathbb{R}^{n} ; \mathbb{R}\right), f \in \mathcal{O}_{M}\left(\mathbb{R}^{n} ; \mathbb{R}\right)$ e $\alpha \in \mathbb{N}^{n}$.

1. A distribuição temperada definida por

$$
\varphi \in \mathcal{S}\left(\mathbb{R}^{n} ; \mathbb{R}\right) \longmapsto T(f \varphi)
$$

é chamada de produto de $f$ por $T$ e é denotada por $f T$.

2. A distribuição temperada definida por

$$
\varphi \in \mathcal{S}\left(\mathbb{R}^{n} ; \mathbb{R}\right) \longmapsto(-1)^{|\alpha|} T\left(\partial^{\alpha} \varphi\right)
$$

é chamada de derivada parcial de ordem $|\alpha|$ de $T$ e é denotada por $\partial^{\alpha} T$.

Para apresentar uma imersão de $\mathcal{S}^{\prime}\left(\mathbb{R}^{n} ; \mathbb{R}\right)$ em $\mathcal{G}_{\tau}\left(\mathbb{R}^{n} ; \mathbb{R}\right)$ utilizaremos o produto de convolução entre uma distribuição temperada e um elemento de $\mathcal{S}\left(\mathbb{R}^{n} ; \mathbb{R}\right)$. Esse produto é definido da seguinte forma.

Definição 2.1.24 Sejam $T \in \mathcal{S}^{\prime}\left(\mathbb{R}^{n} ; \mathbb{R}\right)$ e $\varphi \in \mathcal{S}\left(\mathbb{R}^{n} ; \mathbb{R}\right)$. A aplicação definida por:

$$
x \in \mathbb{R}^{n} \longmapsto T(\varphi(x-\bullet))
$$

é chamada de produto de convolução entre $T$ e $\varphi$, e é denotada por $T * \varphi$.

Observe que, para $\psi \in \mathscr{C}_{c}^{\infty}\left(\mathbb{R}^{n} ; \mathbb{R}\right)$ e $T \in \mathcal{S}^{\prime}\left(\mathbb{R}^{n} ; \mathbb{R}\right)$, tem-se $\left.T\right|_{\mathscr{C}_{c}^{\infty}\left(R^{n} ; R\right)} * \psi$ é o produto de convolução definido na Definição 2.1.9. Note ainda que o produto de convolução tem as seguintes propriedades cujas provas podem ser encontradas, por exemplo em [Bar].

$$
\begin{gathered}
S e \quad \varphi, \psi \in \mathcal{S}\left(\mathbb{R}^{n} ; \mathbb{R}\right), \quad T \in \mathcal{S}^{\prime}\left(\mathbb{R}^{n} ; \mathbb{R}\right) \quad \text { e } \alpha \in \mathbb{N}^{n}, \quad \text { então } \\
T * \varphi \in \mathcal{S}\left(\mathbb{R}^{n} ; \mathbb{R}\right) ; \\
(T * \varphi) * \psi=T *(\varphi * \psi) ;
\end{gathered}
$$




$$
\partial^{\alpha}(T * \varphi)=T *\left(\partial^{\alpha} \varphi\right) .
$$

Encerramos esta seção destacando o seguinte resultado que será muito importante para obtermos uma imersão de $\mathcal{S}^{\prime}\left(\mathbb{R}^{n} ; \mathbb{R}\right)$ em $\mathcal{G}_{\tau}\left(\mathbb{R}^{n} ; \mathbb{R}\right)$ e cuja prova pode ser encontrada, por exemplo, em [Bar].

Seja $T \in \mathcal{S}^{\prime}\left(\mathbb{R}^{n} ; \mathbb{R}\right)$. Então existem $\alpha \in \mathbb{N}^{n}, m \in \mathbb{N}$ e $f \in \mathscr{C}\left(\mathbb{R}^{n} ; \mathbb{R}\right)$ tais que

$$
f \text { é limitada e } T=\partial^{\alpha}\left(d_{(1+|x|)^{m} f}\right)
$$

onde $d_{(1+|x|)^{m} f}$ é a distribuição temperada definida em (2.16) substitutindo $f$ pela função $x \in \mathbb{R}^{n} \longmapsto(1+|x|)^{m} f(x)$. Além disso, tem-se que

$$
T * \varphi \in \mathcal{O}_{M}\left(\mathbb{R}^{n} ; \mathbb{R}\right), \quad \forall \varphi \in \mathcal{S}\left(\mathbb{R}^{n} ; \mathbb{R}\right)
$$

\subsection{Uma imersão de $\mathcal{D}^{\prime}(\Omega ; \mathbb{R})$ em $\mathcal{G}(\Omega ; \mathbb{R})$}

Esta seção é dedicada à construção de uma imersão de $\mathcal{D}^{\prime}(\Omega ; \mathbb{R})$ em $\mathcal{G}(\Omega ; \mathbb{R})$, onde $\Omega$ é um aberto de $\mathbb{R}^{n}$. A imersão que apresentamos é basicamente a descrita por A. Delcroix em [Del]. A diferença entre a imersão obtida por A. Delcroix e a aqui encontrada é a substituição de $\ln \varepsilon$ por $\sqrt{\varepsilon}$ na função $\theta_{\varepsilon}$ descrita a seguir. Essa mudança foi motivada pela construção, encontrada em [GKOS], de um representante da distribuição temperada $\delta$ como função generalizada temperada.

Para atingir o nosso objetivo, definiremos duas famílias de funções que serão muito úteis para a construção da imersão de $\mathcal{D}^{\prime}(\Omega ; \mathbb{R})$ em $\mathcal{G}(\Omega ; \mathbb{R})$, onde $\Omega$ é um aberto de $\mathbb{R}^{n}$. 
Sejam $\rho \in \mathcal{S}\left(\mathbb{R}^{n} ; \mathbb{R}\right)$ e $\chi \in \mathscr{C}_{c}^{\infty}\left(\mathbb{R}^{n} ; \mathbb{R}\right)$ tais que

$$
\begin{gathered}
\int_{R^{n}} \rho(x) d x=1 ; \\
\int_{R^{n}} x^{p} \rho(x) d x=0, \quad \forall p \in \mathbb{N}^{n} \backslash\{0\} ; \\
0 \leq \chi \leq 1 ; \\
\chi(x)=1, \quad \forall x \in B_{1}(0) ; \\
\chi(x)=0, \quad \forall x \in \mathbb{R}^{n} \backslash B_{2}(0) .
\end{gathered}
$$

Para todo $\varepsilon \in \mathbf{I}$, denotaremos por $\rho_{\varepsilon}$ e $\theta_{\varepsilon}$ as funções:

$$
\begin{gathered}
\rho_{\varepsilon}(x):=\frac{1}{\varepsilon^{n}} \rho\left(\frac{x}{\varepsilon}\right), \quad \forall x \in \mathbb{R}^{n} ; \\
\theta_{\varepsilon}(x):=\rho_{\varepsilon}(x) \chi\left(\frac{x}{\sqrt{\varepsilon}}\right), \quad \forall x \in \mathbb{R}^{n} .
\end{gathered}
$$

É claro que $\left(\rho_{\varepsilon}\right)_{\varepsilon}$ e $\left(\theta_{\varepsilon}\right)_{\varepsilon}$ pertencem a $\left(\mathscr{C}^{\infty}\left(\mathbb{R}^{n} ; \mathbb{R}\right)\right)^{\mathbf{I}}$.

Destacaremos, a seguir, algumas propriedades de $\left(\rho_{\varepsilon}\right)_{\varepsilon}$ e de $\left(\theta_{\varepsilon}\right)_{\varepsilon}$ que serão utilizadas no desenvolvimento deste capítulo.

Notemos que, através de uma mudança de variável, tem-se, para todo $\varepsilon \in \mathbf{I}$, que

$$
\begin{gathered}
\int_{R^{n}} \rho_{\varepsilon}(x) d x=1 \\
\int_{R^{n}} x^{p} \rho_{\varepsilon}(x) d x=0, \quad \forall p \in \mathbb{N}^{n} \backslash\{0\} .
\end{gathered}
$$

Em relação a família $\left(\theta_{\varepsilon}\right)_{\varepsilon}$ observaremos um número maior de propriedades. Primeiro, notemos que, para todo $\alpha \in \mathbb{N}^{n}$, tem-se que supp $\partial^{\alpha} \theta_{\varepsilon} \subseteq \overline{B_{2 \sqrt{\varepsilon}}(0)}$. 
A Proposição 2.2.1 nos fornecerá estimativas para as derivadas parciais de $\rho_{\varepsilon}$ e $\theta_{\varepsilon}$.

Proposição 2.2.1 São verdadeiras as seguintes afirmações:

1. $\forall \alpha \in \mathbb{N}^{n}, \exists N \in \mathbb{N}$ e $\exists c>0$ tais que

$$
\left|\partial^{\alpha} \rho_{\varepsilon}(x)\right| \leq c(1+|x|)^{N} \varepsilon^{-N}, \quad \forall(\varepsilon, x) \in \mathbf{I} \times \mathbb{R}^{n}
$$

2. $\forall \alpha \in \mathbb{N}^{n}, \exists N \in \mathbb{N} e \exists c>0$ tais que

$$
\left|\partial^{\alpha} \theta_{\varepsilon}(x)\right| \leq c(1+|x|)^{N} \varepsilon^{-N}, \quad \forall(\varepsilon, x) \in \mathbf{I} \times \mathbb{R}^{n}
$$

3. $\left(\theta_{\varepsilon}\right)_{\varepsilon} \in \mathcal{E}_{\tau}\left[\mathbb{R}^{n} ; \mathbb{R}\right] \quad$ e $\left(\rho_{\varepsilon}\right)_{\varepsilon} \in \mathcal{E}_{\tau}\left[\mathbb{R}^{n} ; \mathbb{R}\right]$;

4. $\left(\theta_{\varepsilon}\right)_{\varepsilon} \in \mathcal{E}_{M}\left[\mathbb{R}^{n} ; \mathbb{R}\right] \quad$ e $\left(\rho_{\varepsilon}\right)_{\varepsilon} \in \mathcal{E}_{M}\left[\mathbb{R}^{n} ; \mathbb{R}\right]$.

Demonstração: Notemos que 3. é conseqüência das afirmações 1. e 2., e que 4. segue de 3. e da Proposição 1.3.12 (1.). Provaremos, a seguir, 1. e 2..

Fixemos $\alpha \in \mathbb{N}^{n}$.

Para mostrarmos 1., observemos que, do fato de $\rho \in \mathcal{S}\left(\mathbb{R}^{n} ; \mathbb{R}\right)$, existe $c_{1}>0$ tal que

$$
\left|\partial^{\alpha} \rho(x)\right| \leq c_{1}(1+|x|)^{-1}, \quad \forall x \in \mathbb{R}^{n}
$$

Logo, para todo $(\varepsilon, x) \in \mathbf{I} \times \mathbb{R}^{n}$, temos que

$$
\begin{aligned}
\left|\partial^{\alpha} \rho_{\varepsilon}(x)\right| & =\left|\partial^{\alpha}\left(\frac{1}{\varepsilon^{n}} \rho\left(\frac{x}{\varepsilon}\right)\right)\right| \\
& =\left|\partial^{\alpha} \rho\left(\frac{x}{\varepsilon}\right)\right| \varepsilon^{-n-|\alpha|} \\
& \leq c_{1}\left(1+\left|\frac{x}{\varepsilon}\right|\right)^{-1} \varepsilon^{-n-|\alpha|} \\
& \leq c_{1}(1+|x|)^{-1} \varepsilon^{-n-|\alpha|} \\
& \leq c_{1}(1+|x|)^{n+|\alpha|} \varepsilon^{-n-|\alpha|}
\end{aligned}
$$

Seja $N:=n+|\alpha|$. Logo, temos que

$$
\left|\partial^{\alpha} \rho_{\varepsilon}(x)\right| \leq c_{1}(1+|x|)^{N} \varepsilon^{-N}, \quad \forall(\varepsilon, x) \in \mathbf{I} \times \mathbb{R}^{n},
$$


o que prova $1 .$.

Para 2. notemos que

$$
\partial^{\alpha} \theta_{\varepsilon}(x)=\sum_{0 \leq \beta \leq \alpha}\left(\begin{array}{l}
\alpha \\
\beta
\end{array}\right) \varepsilon^{-n-|\beta|} \partial^{\beta} \rho\left(\frac{x}{\varepsilon}\right) \partial^{\alpha-\beta} \chi\left(\frac{x}{\sqrt{\varepsilon}}\right) \varepsilon^{(|\beta|-|\alpha|) / 2},
$$

para todo $(\varepsilon, x) \in \mathbf{I} \times \mathbb{R}^{n}$.

Fixemos $\beta \in \mathbb{N}^{n}$ com $0 \leq \beta \leq \alpha$. Como $\rho \in \mathcal{S}\left(\mathbb{R}^{n} ; \mathbb{R}\right)$ e $\chi \in \mathscr{C}_{c}^{\infty}\left(\mathbb{R}^{n} ; \mathbb{R}\right)$ existe $c_{\beta}>0$ tal que

$$
\begin{gathered}
\left|\partial^{\beta} \rho(y)\right| \leq c_{\beta}(1+|y|)^{-1}, \quad \forall y \in \mathbb{R}^{n} \\
\left|\partial^{\alpha-\beta} \chi(y)\right| \leq c_{\beta}, \quad \forall y \in \mathbb{R}^{n} .
\end{gathered}
$$

A partir dessas desigualdades temos

$$
\begin{aligned}
\left|\partial^{\alpha} \theta_{\varepsilon}(x)\right| & \leq \sum_{0 \leq \beta \leq \alpha}\left(\begin{array}{l}
\alpha \\
\beta
\end{array}\right) c_{\beta}\left(1+\left|\frac{x}{\varepsilon}\right|\right)^{-1} c_{\beta} \varepsilon^{-(n+|\alpha|)} \\
& \leq \sum_{0 \leq \beta \leq \alpha}\left(\begin{array}{l}
\alpha \\
\beta
\end{array}\right)\left(c_{\beta}\right)^{2}(1+|x|)^{-1} \varepsilon^{-(n+|\alpha|)} \\
& \leq \sum_{0 \leq \beta \leq \alpha}\left(\begin{array}{l}
\alpha \\
\beta
\end{array}\right)\left(c_{\beta}\right)^{2}(1+|x|)^{n+|\alpha|} \varepsilon^{-(n+|\alpha|)},
\end{aligned}
$$

para todo $(\varepsilon, x) \in \mathbf{I} \times \mathbb{R}^{n}$

$$
\begin{aligned}
& \text { Sejam } N_{1}:=n+|\alpha| \text { e } c:=\sum_{0 \leq \beta \leq \alpha}\left(\begin{array}{l}
\alpha \\
\beta
\end{array}\right)\left(c_{\beta}\right)^{2} \text {. Então } \\
& \quad\left|\partial^{\alpha} \theta_{\varepsilon}(x)\right| \leq c(1+|x|)^{N_{1}} \varepsilon^{-N_{1}}, \quad \forall(\varepsilon, x) \in \mathbf{I} \times \mathbb{R}^{n},
\end{aligned}
$$

o que prova 2.

Na Proposição 2.2.3 destacaremos mais algumas propriedades de $\left(\theta_{\varepsilon}\right)_{\varepsilon}$ que também serão muito úteis no nosso trabalho. Para tal utilizaremos o seguinte resultado.

Proposição 2.2.2 São verdadeiras as seguintes afirmações:

1. $\forall m \in \mathbb{N}, \exists c>0 \quad$ tal que $\left|\rho_{\varepsilon}(x)-\theta_{\varepsilon}(x)\right| \leq c \varepsilon^{m}, \quad \forall(\varepsilon, x) \in \mathbf{I} \times \mathbb{R}^{n}$; 
2. $\forall m \in \mathbb{N}, \exists N \in \mathbb{N}, \exists c>0$ tais que $\left|\rho_{\varepsilon}(x)-\theta_{\varepsilon}(x)\right| \leq c(1+|x|)^{N} \varepsilon^{m}$, $\forall(\varepsilon, x) \in \mathbf{I} \times \mathbb{R}^{n} ;$

3. $\forall m \in \mathbb{N}, \exists c>0 \quad e \quad \exists \eta \in \mathbf{I} \quad$ tais que $\left|\int_{R^{n}} \theta_{\varepsilon}(x) d x-1\right| \leq c \varepsilon^{m}, \quad \forall \varepsilon \in \mathbf{I}_{\eta}$;

4. $\forall p \in \mathbb{N}^{n} \backslash\{0\}, \forall m \in \mathbb{N}, \exists c>0 \quad$ e $\exists \eta \in \mathbf{I} \quad$ tais que $\left|\int_{R^{n}} x^{p} \theta_{\varepsilon}(x) d x\right| \leq c \varepsilon^{m}$, $\forall \varepsilon \in \mathbf{I}_{\eta}$.

Demonstração: Note que para 2. basta escolher $N=0$ e usar 1.. Antes de provarmos as outras afirmações notemos que

$$
\left|\rho_{\varepsilon}(x)-\theta_{\varepsilon}(x)\right|=\left|\rho_{\varepsilon}(x)\right|\left|1-\chi\left(\frac{x}{\sqrt{\varepsilon}}\right)\right| \leq\left|\rho_{\varepsilon}(x)\right|, \quad \forall(\varepsilon, x) \in \mathbf{I} \times \mathbb{R}^{n},
$$

onde a última desigualdade decorre de $0 \leq \chi \leq 1$. Além disso, de $\chi \equiv 1$ em $B_{1}(0)$, temos

$$
\left|\rho_{\varepsilon}(x)-\theta_{\varepsilon}(x)\right|=0, \quad \forall x \in B_{\sqrt{\varepsilon}}(0)
$$

e, do fato de $\rho \in \mathcal{S}\left(\mathbb{R}^{n} ; \mathbb{R}\right)$, temos que, para todo $q \in \mathbb{N}$, existe $c_{1}>0$ tal que

$$
|\rho(y)| \leq c_{1}(1+|y|)^{-q}, \quad \forall y \in \mathbb{R}^{n}
$$

Seja $m \in \mathbb{N}$.

Para obtermos 1 , considere $q:=2(m+n+1)$ e seja $c:=c_{1}$, onde $c_{1}>0$ é como em $(2.31)$

Fixemos $\varepsilon \in$ I. Então, para todo $x \notin B_{\sqrt{\varepsilon}}(0)$ temos, por (2.29) e (2.31) que

$$
\begin{aligned}
\left|\rho_{\varepsilon}(x)-\theta_{\varepsilon}(x)\right| & \leq\left|\rho_{\varepsilon}(x)\right| \\
& \leq \frac{1}{\varepsilon^{n}}\left|\rho\left(\frac{x}{\varepsilon}\right)\right| \\
& \leq c_{1} \varepsilon^{-n}\left(1+\left|\frac{x}{\varepsilon}\right|\right)^{-q} \\
& \leq c_{1} \varepsilon^{-n+q}(\varepsilon+|x|)^{-q} \\
& \leq c_{1} \varepsilon^{-n+q}(\varepsilon+\sqrt{\varepsilon})^{-q} \\
& \leq 2^{-q} c_{1} \varepsilon^{q / 2-n} \\
& \leq c \varepsilon^{m} .
\end{aligned}
$$


Logo, desse fato e de (2.30), temos que

$$
\left|\rho_{\varepsilon}(x)-\theta_{\varepsilon}(x)\right| \leq c \varepsilon^{m}, \quad \forall(\varepsilon, x) \in \mathbf{I} \times \mathbb{R}^{n},
$$

o que prova 1 ..

Provaremos a seguir que, para todo $p \in \mathbb{N}^{n}$ existe $c>0$ tal que

$$
\left|\int_{R^{n}} x^{p}\left(\theta_{\varepsilon}(x)-\rho_{\varepsilon}(x)\right) d x\right| \leq c \varepsilon^{m}, \quad \forall \varepsilon \in \mathbf{I},
$$

e assim, por (2.26) e (2.27), teremos 3. e 4..

Fixemos $p \in \mathbb{N}$ e $\varepsilon \in \mathbf{I}$. Então, por (2.29) e (2.30), temos

$$
\begin{aligned}
\left|\int_{R^{n}} x^{p}\left(\theta_{\varepsilon}(x)-\rho_{\varepsilon}(x)\right) d x\right| & =\left|\int_{R^{n} \backslash B_{\sqrt{\varepsilon}}(0)} x^{p}\left(\theta_{\varepsilon}(x)-\rho_{\varepsilon}(x)\right) d x+\int_{B_{\sqrt{\varepsilon}}(0)} x^{p}\left(\theta_{\varepsilon}(x)-\rho_{\varepsilon}(x)\right) d x\right| \\
& =\left|\int_{R^{n} \backslash B_{\sqrt{\varepsilon}}(0)} x^{p}\left(\theta_{\varepsilon}(x)-\rho_{\varepsilon}(x)\right) d x\right| \\
& \leq \int_{R^{n} \backslash B_{\sqrt{\varepsilon}}(0)}\left|x^{p}\right|\left|\theta_{\varepsilon}(x)-\rho_{\varepsilon}(x)\right| d x \\
& \leq \int_{R^{n} \backslash B_{\sqrt{\varepsilon}}(0)}\left|x^{p}\right|\left|\rho_{\varepsilon}(x)\right| d x .
\end{aligned}
$$

Considere $q:=\max \{p+2 n, m+2 n\}$ e seja $c:=\frac{c_{1}(2 \pi)^{n-1}}{n}$, onde $c_{1}>0$ é como em (2.31). Dessa forma temos que

$$
\begin{aligned}
\left|\int_{R^{n}} x^{p}\left(\theta_{\varepsilon}(x)-\rho_{\varepsilon}(x)\right) d x\right| & \leq \int_{R^{n} \backslash B_{\sqrt{\varepsilon}}(0)}\left|x^{p}\right| \frac{1}{\varepsilon^{n}}\left|\rho\left(\frac{x}{\varepsilon}\right)\right| \\
& \leq \int_{R^{n} \backslash B_{\sqrt{\varepsilon}}(0)} c_{1}\left|x^{p}\right|\left(1+\left|\frac{x}{\varepsilon}\right|\right)^{-q} \varepsilon^{-n} d x \\
& \leq \int_{R^{n} \backslash B_{\sqrt{\varepsilon}}(0)} c_{1} \varepsilon^{-n+q} \frac{|x|^{p}}{(\varepsilon+|x|)^{q}} d x \\
& \leq \int_{R^{n} \backslash B_{\sqrt{\varepsilon}}(0)} c_{1} \varepsilon^{-n+q}|x|^{p-q} d x \\
& \leq \int_{R^{n} \backslash B_{\sqrt{\varepsilon}}(0)} \frac{c_{1} \varepsilon^{-n+q}}{|x|^{2 n}} d x
\end{aligned}
$$




$$
\begin{aligned}
& \leq \frac{c_{1} \varepsilon^{-n+q}(2 \pi)^{n-1}}{n(\sqrt{\varepsilon})^{n}} \\
& \leq c \varepsilon^{m}
\end{aligned}
$$

o que prova (2.32).

Proposição 2.2.3 São verdadeiras as seguintes afirmações:

1. $\left(\rho_{\varepsilon}-\theta_{\varepsilon}\right)_{\varepsilon} \in \mathcal{N}\left[\mathbb{R}^{n} ; \mathbb{R}\right]$

2. $\left(\rho_{\varepsilon}-\theta_{\varepsilon}\right)_{\varepsilon} \in \mathcal{N}_{\tau}\left[\mathbb{R}^{n} ; \mathbb{R}\right]$;

3. $\left(\int_{R^{n}} \theta_{\varepsilon}(x) d x-1\right)_{\varepsilon} \in \mathcal{N}(\mathbb{R})$;

4. $\left(\int_{R^{n}} x^{p} \theta_{\varepsilon}(x) d x\right)_{\varepsilon} \in \mathcal{N}(\mathbb{R}), \quad \forall p \in \mathbb{N}^{n} \backslash\{0\}$.

Demonstração: Todas as asserções são conseqüências imediatas da Proposição 2.2 .1 (3.) e (4.), da Proposição 2.2 .2 e dos teoremas de nulidade em $\mathcal{E}_{M}\left[\mathbb{R}^{n} ; \mathbb{R}\right]$ e em $\mathcal{E}_{\tau}\left[\mathbb{R}^{n} ; \mathbb{R}\right]$ (Teorema 1.2.5 e Teorema 1.3.7).

Além das famílias $\left(\rho_{\varepsilon}\right)_{\varepsilon}$ e $\left(\theta_{\varepsilon}\right)_{\varepsilon}$, precisaremos considerar uma família $\left(K_{\varepsilon}\right)_{\varepsilon}$ de subconjuntos de $\Omega$, que será descrita a seguir, e de uma terceira família de funções que será construída a partir de $\left(K_{\varepsilon}\right)_{\varepsilon}$.

Seja $\Omega$ um aberto de $\mathbb{R}^{n}$. Defina, para todo $\varepsilon \in \mathbf{I}$,

$$
\begin{gathered}
K_{\varepsilon}:=\left\{x \in \Omega \mid d\left(x, \mathbb{R}^{n} \backslash \Omega\right) \geq \varepsilon \text { e }|x| \leq \frac{1}{\varepsilon}\right\}, \text { se } \Omega \neq \mathbb{R}^{n}, \\
K_{\varepsilon}:=\left\{x \in \mathbb{R}^{n}|| x \mid \leq \frac{1}{\varepsilon}\right\}, \text { se } \Omega=\mathbb{R}^{n} . \\
\text { Seja }\left(\psi_{\varepsilon}\right)_{\varepsilon} \in\left(\mathscr{C}_{c}^{\infty}(\Omega ; \mathbb{R}) \cup\{0\}\right)^{\mathbf{I}} \text { tal que, para todo } \varepsilon \in \mathbf{I}, \text { tem-se } \\
0 \leq \psi_{\varepsilon} \leq 1 ; \psi_{\varepsilon} \equiv 1 \text { em } K_{\varepsilon} \text { se } K_{\varepsilon} \neq \emptyset ; \quad \psi_{\varepsilon} \equiv 0 \text { se } K_{\varepsilon}=\emptyset .
\end{gathered}
$$


No ínicio deste capítulo, observamos que existe $\left(\psi_{\varepsilon}\right)_{\varepsilon}$ como em (2.35).

Em toda esta seção e na próxima, $\left(\rho_{\varepsilon}\right)_{\varepsilon},\left(\theta_{\varepsilon}\right)_{\varepsilon}$ e $\left(\psi_{\varepsilon}\right)_{\varepsilon}$ denotarão as famílias consideradas em (2.24), (2.25) e (2.35) e, para todo $\varepsilon \in \mathbf{I}, K_{\varepsilon}$ denotará o conjunto descrito em (2.33) ou $(2.34)$.

Convém observar o seguinte.

Observação 2.2.4 Seja $\Omega$ um aberto de $\mathbb{R}^{n}$. Então

1. $K_{\varepsilon} \subset \subset \Omega, \quad \forall \varepsilon \in \mathbf{I}$;

2. $\exists \eta \in \mathbf{I}$ tal que $K_{\varepsilon} \neq \emptyset, \quad \forall \varepsilon \in \mathbf{I}_{\eta}$;

3. $\forall K \subset \subset \Omega, \exists \eta \in \mathbf{I}$ tal que $K \subset K_{\varepsilon}, \quad \forall \varepsilon \in \mathbf{I}_{\eta}$;

4. $\forall K \subset \subset \Omega, \quad \forall L$ aberto de $\mathbb{R}^{n}$ tal que $K \subset L \subset \bar{L} \subset \subset \Omega, \exists \eta_{1} \in \mathbf{I}, \exists \nu>0$ tais que

$$
\begin{gathered}
K \subset L \subset K_{\varepsilon}, \quad \forall \varepsilon \in \mathbf{I}_{\eta_{1}} ; \\
\operatorname{supp}_{\varepsilon}(x-\bullet) \subset \overline{B_{\nu}(x)} \subset L, \quad \forall(\varepsilon, x) \in \mathbf{I}_{\eta_{1}} \times K .
\end{gathered}
$$

De fato, 1. e 2. decorrem da definição de $K_{\varepsilon}$. Para 3. basta considerar $b_{1}:=\max \{|x| \mid x \in K\} \quad$ e $\quad$ definir $\quad \eta:=\min \left\{1, \frac{1}{b_{1}}\right\} \quad$ se $\quad \Omega=\mathbb{R}^{n} \mathrm{e}$ $\eta:=\min \left\{1, \frac{1}{b_{1}}, d\left(K, \mathbb{R}^{n} \backslash \Omega\right)\right\}$ se $\Omega \neq \mathbb{R}^{n}$. Para 4. basta considerar $\eta_{1}:=\min \left\{\eta,\left(\frac{\nu}{2}\right)^{2}\right\}$, onde $\nu:=\frac{1}{2} d\left(K, \mathbb{R}^{n} \backslash L\right)$ e $\eta$ é como em 3. substituindo $K$ por $\bar{L}$.

Após termos observado algumas propriedades das famílias $\left(\rho_{\varepsilon}\right)_{\varepsilon}$ e $\left(\theta_{\varepsilon}\right)_{\varepsilon}$, iremos apresentar alguns resultados que nos serão úteis para obtermos uma imersão de $\mathcal{D}^{\prime}(\Omega ; \mathbb{R})$ em $\mathcal{G}(\Omega ; \mathbb{R})$, onde $\Omega$ é um aberto de $\mathbb{R}^{n}$, que é o objetivo desta seção. Iniciaremos observando o seguinte. 
Observação 2.2.5 Sejam $\Omega$ um aberto de $\mathbb{R}^{n}, \varepsilon \in \mathbf{I}$ e $T \in \mathcal{D}^{\prime}(\Omega ; \mathbb{R})$. Então a aplicação

$$
T_{\varepsilon}: \varphi \in \mathscr{C}_{c}^{\infty}\left(\mathbb{R}^{n} ; \mathbb{R}\right) \longmapsto T\left(\left.\psi_{\varepsilon} \varphi\right|_{\Omega}\right)
$$

é uma distribuição sobre $\mathbb{R}^{n}$, onde $\psi_{\varepsilon}$ é como em (2.35).

De fato, basta usar o Teorema 2.1 .5 e observar que, do fato de $\psi_{\varepsilon} \in \mathscr{C}_{c}^{\infty}(\Omega ; \mathbb{R})$ tem-se, se $\varphi_{\tau} \rightarrow 0$ em $\mathscr{C}_{c}^{\infty}\left(\mathbb{R}^{n} ; \mathbb{R}\right)$, quando $\tau \downarrow 0$, então $\left.\psi_{\varepsilon} \varphi_{\tau}\right|_{\Omega} \rightarrow 0$ em $\mathscr{C}_{c}^{\infty}(\Omega ; \mathbb{R})$, quando $\tau \downarrow 0$.

Note que, se $\varphi \in \mathscr{C}_{c}^{\infty}(\Omega ; \mathbb{R})$, $\varphi^{*}$ é a função definida em $\mathbb{R}^{n}$ por

$$
\varphi^{*}(x)=\left\{\begin{array}{lll}
\varphi(x) & \text { se } & x \in W \\
0 & \text { se } & x \notin W
\end{array},\right.
$$

onde $W$ é um aberto de $\mathbb{R}^{n}$ tal que supp $\varphi \subset W \subset \Omega$, e se $T_{\varepsilon}$ é como em (2.36), então

$$
\left.T_{\varepsilon}\right|_{\Omega}\left(\varphi^{*}\right)=T_{\varepsilon}\left(\varphi^{*}\right)=T\left(\left.\psi_{\varepsilon} \varphi^{*}\right|_{\Omega}\right)=T\left(\psi_{\varepsilon} \varphi\right)=\left(\psi_{\varepsilon} T\right)(\varphi) \text {, }
$$

onde $\psi_{\varepsilon} T$ é como na Definição 2.1 .8 (1.). Por esse motivo escreveremos $\psi_{\varepsilon} T$ no lugar de $T_{\varepsilon}$, ou seja,

$$
\left(\psi_{\varepsilon} T\right)(\varphi):=T\left(\left.\psi_{\varepsilon} \varphi\right|_{\Omega}\right), \quad \forall \varphi \in \mathscr{C}_{c}^{\infty}\left(\mathbb{R}^{n} ; \mathbb{R}\right) .
$$

Observe ainda que, de (2.10), (2.11) e (2.12) tem-se

$$
\begin{gathered}
\psi_{\varepsilon} T * \theta_{\varepsilon} \in \mathscr{C}^{\infty}\left(\mathbb{R}^{n} ; \mathbb{R}\right) ; \\
\left(\psi_{\varepsilon} T * \varphi\right) * \psi=\psi_{\varepsilon} T *(\varphi * \psi), \quad \forall \varphi, \psi \in \mathscr{C}_{c}^{\infty}\left(\mathbb{R}^{n} ; \mathbb{R}\right) ; \\
\partial^{\alpha}\left(\psi_{\varepsilon} T * \varphi\right)=\psi_{\varepsilon} T *\left(\partial^{\alpha} \varphi\right), \quad \forall \varphi \in \mathscr{C}_{c}^{\infty}\left(\mathbb{R}^{n} ; \mathbb{R}\right), \quad \forall \alpha \in \mathbb{N}^{n},
\end{gathered}
$$

para todo $\varepsilon \in \mathbf{I}$.

De (2.38), para todo $\varepsilon \in \mathbf{I}$, podemos definir, sobre $\Omega$, a distribuição $d_{\psi_{\varepsilon} T * \theta_{\varepsilon}}$. Na Proposição 2.2.6, estudaremos o comportamento de $\left(d_{\psi_{\varepsilon} T * \theta_{\varepsilon}}\right)_{\varepsilon}$, quando $\varepsilon \downarrow 0$. 
Proposição 2.2.6 Sejam $\Omega$ um aberto de $\mathbb{R}^{n}$ e $T \in \mathcal{D}^{\prime}(\Omega ; \mathbb{R})$. Então

$$
d_{\psi_{\varepsilon} T * \theta_{\varepsilon}} \longrightarrow T e m \mathcal{D}^{\prime}(\Omega ; \mathbb{R}), \text { quando } \varepsilon \downarrow 0,
$$

onde $d_{\psi_{\varepsilon} T * \theta_{\varepsilon}}$ é a distribuição sobre $\Omega$ definida em (2.6).

Demonstração: Antes de iniciarmos a prova introduziremos a seguinte notação.

Se $g \in \mathscr{C}^{\infty}\left(\mathbb{R}^{n} ; \mathbb{R}\right)$, denotaremos por $g^{\nu}$ a função definida em $\mathbb{R}^{n}$ por

$$
g^{\nu}(x):=g(-x), \quad \forall x \in \mathbb{R}^{n}
$$

Seja $\varphi \in \mathscr{C}_{c}^{\infty}(\Omega ; \mathbb{R})$. Provaremos que $\lim _{\varepsilon \downarrow 0} d_{\psi_{\varepsilon} T * \theta_{\varepsilon}}(\varphi)=T(\varphi)$. Para isso, considere $\Phi$ a função definida em $\mathbb{R}^{n}$ por

$$
\Phi(x)=\left\{\begin{array}{lll}
\varphi(x) & \text { se } & x \in W \\
0 & \text { se } & x \notin W
\end{array},\right.
$$

onde $W$ é um aberto de $\mathbb{R}^{n}$ tal que $\operatorname{supp} \varphi \subset W \subset \Omega$.

Notemos que, para $\varepsilon \in \mathbf{I}$,

$$
d_{\psi_{\varepsilon} T * \theta_{\varepsilon}}(\varphi)=\int_{\Omega}\left(\psi_{\varepsilon} T * \theta_{\varepsilon}\right)(y) \varphi(y) d y=\int_{R^{n}}\left(\psi_{\varepsilon} T * \theta_{\varepsilon}\right)(y) \Phi(y) d y
$$

ou seja,

$$
d_{\psi_{\varepsilon} T * \theta_{\varepsilon}}(\varphi)=\int_{R^{n}}\left(\psi_{\varepsilon} T * \theta_{\varepsilon}\right)(y) \Phi^{\nu}(0-y) d y=\left(\left(\psi_{\varepsilon} T * \theta_{\varepsilon}\right) * \Phi^{\nu}\right)(0) .
$$

Logo, por (2.39), temos que

$$
d_{\psi_{\varepsilon} T * \theta_{\varepsilon}}(\varphi)=\left(\left(\psi_{\varepsilon} T * \theta\right)_{\varepsilon} * \Phi^{\nu}\right)(0)=\left(\psi_{\varepsilon} T *\left(\theta_{\varepsilon} * \Phi^{\nu}\right)\right)(0)=\psi_{\varepsilon} T\left(\left(\theta_{\varepsilon} * \Phi^{\nu}\right)^{\nu}\right),
$$

isto é

$$
d_{\psi_{\varepsilon} T * \theta_{\varepsilon}}(\varphi)=T\left(\left.\psi_{\varepsilon}\left(\theta_{\varepsilon} * \Phi^{\nu}\right)^{\nu}\right|_{\Omega}\right), \quad \forall \varepsilon \in \mathbf{I} .
$$

Seja $K=\operatorname{supp} \Phi^{\nu}$. Note que $\operatorname{supp} \varphi=\operatorname{supp} \Phi=\left\{x \in \mathbb{R}^{n} \mid-x \in K\right\}$. Considere $L$ aberto de $\mathbb{R}^{n}$ tal que

$$
K \subset L \subset \bar{L} \subset \subset\left\{x \in \mathbb{R}^{n} \mid-x \in \Omega\right\}=:-\Omega
$$


e $s:=\frac{1}{4} d(-\Omega \backslash L ; K)$.

De $K$ ser compacto e $K \subset \bigcup_{x \in K} B_{s}(x)$ existem $x_{1}, \cdots, x_{l} \in K$ tais que

$$
K \subset \bigcup_{i=1}^{l} B_{s}\left(x_{i}\right) \subset \bigcup_{i=1}^{l} \overline{B_{2 s}\left(x_{i}\right)} \subset L .
$$

$$
\begin{aligned}
& \text { Seja } \eta_{1}:=\min \left\{1, \frac{s^{2}}{4}\right\} \text {. Então } \\
& \operatorname{supp}\left(\theta_{\varepsilon} * \Phi^{\nu}\right) \subset \operatorname{supp} \theta_{\varepsilon}+\operatorname{supp} \Phi^{\nu} \subset \overline{B_{2 \sqrt{\varepsilon}}(0)}+K \subset L, \quad \forall \varepsilon \in \mathbf{I}_{\eta_{1}},
\end{aligned}
$$

onde a primeira inclusão decorre de (2.1) e a última segue de, se $y \in K$, então, por (2.42), existe $1 \leq i \leq l$ tal que $\left|y-x_{i}\right|<s$, e assim

$$
\left|x+y-x_{i}\right| \leq|x|+\left|y-x_{i}\right| \leq 2 \sqrt{\varepsilon}+s<2 \frac{s}{2}+s=2 s, \quad \forall x \in \overline{B_{2 \sqrt{\varepsilon}}(0)}
$$

Seja $V:=\left\{x \in \mathbb{R}^{n} \mid-x \in L\right\}$. Então $V$ é um aberto de $\Omega$ e, por (2.43),

$$
\operatorname{supp}\left(\theta_{\varepsilon} * \Phi^{\nu}\right)^{\nu} \subset V \subset \bar{V} \subset \subset \Omega, \quad \forall \varepsilon \in \mathbf{I}_{\eta_{1}} \text {. }
$$

Considere $\eta_{2} \in \mathbf{I}$ com $\eta_{2}<\eta_{1}$ tal que $\bar{V} \subset K_{\varepsilon}$ para todo $\varepsilon \in \mathbf{I}_{\eta_{2}}$ (Observação 2.2.4 (3.)). Então, de (2.35), (2.41) e (2.44), temos que

$$
d_{\psi_{\varepsilon} T * \theta_{\varepsilon}}(\varphi)=\left.T\right|_{V}\left(\left.\psi_{\varepsilon}\left(\theta_{\varepsilon} * \Phi^{\nu}\right)^{\nu}\right|_{\Omega}\right)=\left.T\right|_{V}\left(\left.\left(\theta_{\varepsilon} * \Phi^{\nu}\right)^{\nu}\right|_{\Omega}\right)=T\left(\left.\left(\theta_{\varepsilon} * \Phi^{\nu}\right)^{\nu}\right|_{\Omega}\right),
$$

para todo $\varepsilon \in \mathbf{I}_{\eta_{2}}$.

Seja $\left(g_{\varepsilon}\right)_{\varepsilon}$ a família de elementos de $\mathscr{C}_{c}^{\infty}(\Omega ; \mathbb{R})$ definida por

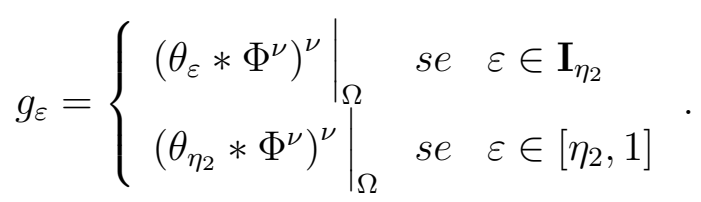

Provaremos, a seguir, que

$$
g_{\varepsilon} \rightarrow \varphi \mathrm{em} \mathscr{C}_{c}^{\infty}(\Omega ; \mathbb{R}), \text { quando } \varepsilon \downarrow 0,
$$

e assim, por (2.45) e pelo Teorema 2.1.5, teremos que

$$
\lim _{\varepsilon \downarrow 0} d_{\psi_{\varepsilon} T * \theta_{\varepsilon}}(\varphi)=\lim _{\varepsilon \downarrow 0} T\left(g_{\varepsilon}\right)=T(\varphi)
$$


que é o que queremos demonstrar.

Afim de obtermos (2.46) verificaremos, em primeiro lugar, que

$$
\theta_{\varepsilon} * \psi \stackrel{u}{\longrightarrow} \psi \text {, quando } \varepsilon \downarrow 0, \quad \forall \psi \in \mathscr{C}_{c}^{\infty}\left(\mathbb{R}^{n} ; \mathbb{R}\right),
$$

onde $\stackrel{u}{\longrightarrow}$ significa convergência uniforme.

Seja $\psi \in \mathscr{C}_{c}^{\infty}\left(\mathbb{R}^{n} ; \mathbb{R}\right)$. Então

$$
\left|\theta_{\varepsilon} * \psi-\psi\right|(x)=\left|\int_{R^{n}} \psi(x-y) \theta_{\varepsilon}(y) d y-\psi(x)\right|, \quad \forall(\varepsilon, x) \in \mathbf{I} \times \mathbb{R}^{n} .
$$

Da Proposição 2.2.2 (3.), existem $\eta_{3} \in \mathbf{I} \operatorname{com} \eta_{3}<\eta_{2}$ e $c>0$ tais que

$$
\left|1-\int_{R^{n}} \theta_{\varepsilon}(y) d y\right| \leq c \varepsilon, \quad \forall \varepsilon \in \mathbf{I}_{\eta_{3}} .
$$

Logo, para todo $(\varepsilon, t) \in \mathbf{I}_{\eta_{3}} \times \mathbb{R}^{n}$ tem-se

$$
\begin{aligned}
\left|\theta_{\varepsilon} * \psi-\psi\right|(x) & =\left|\int_{R^{n}} \psi(x-y) \theta_{\varepsilon}(y) d y-\psi(x)\left[1-\int_{R^{n}} \theta_{\varepsilon}(y) d y\right]-\int_{R^{n}} \psi(x) \theta_{\varepsilon}(y) d y\right| \\
& \left.\leq \int_{R^{n}} \mid \psi(x-y)-\psi(x)\right] \theta_{\varepsilon}(y)|d y+| \psi(x)|| 1-\int_{R^{n}} \theta_{\varepsilon}(y) d y \mid \\
& \leq \int_{R^{n}}|\psi(x-y)-\psi(x)|\left|\theta_{\varepsilon}(y)\right| d y+c \varepsilon|| \psi \|_{\infty}
\end{aligned}
$$

e assim

$$
\left|\theta_{\varepsilon} * \psi-\psi\right|(x) \leq \int_{R^{n}}|\psi(x-y)-\psi(x)|\left|\theta_{\varepsilon}(y)\right| d y+c \varepsilon\|\psi\|_{\infty} .
$$

Seja $b>0$. Como $\rho \in \mathcal{S}\left(\mathbb{R}^{n} ; \mathbb{R}\right)$ podemos considerar $M:=\int_{R^{n}}|\rho(y)| d y$ (Observação 2.1 .14 (3.)).

De $\psi \in \mathscr{C}_{c}\left(\mathbb{R}^{n} ; \mathbb{R}\right)$ temos que $\psi$ é uniformemente contínua em $\mathbb{R}^{n}$, e assim existe $r>0$ tal que

$$
|\psi(w)-\psi(z)|<\frac{b}{2 M}, \quad \forall w, z \in \mathbb{R}^{n} \operatorname{com}|w-z|<r .
$$

Seja $\eta_{4}:=\min \left\{\eta_{3}, \frac{r^{2}}{4}, \frac{b}{2 c\left(\|\psi\|_{\infty}+1\right)}\right\}$ e observe que

$$
\theta_{\varepsilon}(y)=\rho_{\varepsilon}(y) \chi\left(\frac{y}{\sqrt{\varepsilon}}\right)=0, \quad \forall(\varepsilon, x) \in \mathbf{I}_{\eta_{4}} \times\left(\mathbb{R}^{n} \backslash B_{r}(0)\right),
$$


pois $\chi \equiv 0$ em $\mathbb{R}^{n} \backslash B_{2}(0)$.

Fixe $(\varepsilon, x) \in \mathbf{I}_{\eta_{4}} \times \mathbb{R}^{n}$. Então, por (2.49) e (2.50), tem-se

$$
\begin{aligned}
\int_{R^{n}}|\psi(x-y)-\psi(x)|\left|\theta_{\varepsilon}(y)\right| d y & =\int_{B_{r}(0)}|\psi(x-y)-\psi(x)|\left|\theta_{\varepsilon}(y)\right| d y \\
& \leq \frac{b}{2 M} \int_{B_{r}(0)}\left|\theta_{\varepsilon}(y)\right| d y \\
& \leq \frac{b}{2 M} \int_{R^{n}}\left|\theta_{\varepsilon}(y)\right| d y \\
& \leq \frac{b}{2 M} \int_{R^{n}}\left|\rho_{\varepsilon}(y)\right| d y=\frac{b}{2 M} \int_{R^{n}}|\rho(y)| d y=\frac{b}{2} .
\end{aligned}
$$

Logo,

$$
\int_{R^{n}}|\psi(x-y)-\psi(x)|\left|\theta_{\varepsilon}(y)\right| d y \leq \frac{b}{2}, \quad \forall(\varepsilon, x) \in \mathbf{I}_{\eta_{4}} \times \mathbb{R}^{n} .
$$

De (2.48) e (2.51), temos que

$$
\left|\left(\theta_{\varepsilon} * \psi\right)(x)-\psi(x)\right| \leq \frac{b}{2}+c \varepsilon\|\psi\|_{\infty}<\frac{b}{2}+\frac{b}{2}=b, \quad \forall(\varepsilon, x) \in \mathbf{I}_{\eta_{4}} \times \mathbb{R}^{n} .
$$

Portanto, como $b>0$ é arbitrário, obtemos (2.47).

De (2.47), é claro que

$$
\theta_{\varepsilon} *\left(\partial^{\alpha} \Phi\right)^{\nu} \stackrel{u}{\longrightarrow}\left(\partial^{\alpha} \Phi\right)^{\nu}, \text { quando } \varepsilon \downarrow 0, \quad \forall \alpha \in \mathbb{N}^{n},
$$

e assim

$$
\left(\theta_{\varepsilon} *\left(\partial^{\alpha} \Phi\right)^{\nu}\right)^{\nu} \stackrel{u}{\longrightarrow} \partial^{\alpha} \Phi, \text { quando } \varepsilon \downarrow 0, \quad \forall \alpha \in \mathbb{N}^{n} .
$$

Observando que

$$
\partial^{\alpha}\left(g^{\nu}\right)=(-1)^{|\alpha|}\left(\partial^{\alpha} g\right)^{\nu}, \quad \forall g \in \mathscr{C}^{\infty}\left(\mathbb{R}^{n} ; \mathbb{R}\right)
$$

e usando (2.4) temos que

$\partial^{\alpha}\left[\left(\theta_{\varepsilon} * \Phi^{\nu}\right)^{\nu}\right]=(-1)^{|\alpha|}\left(\partial^{\alpha}\left(\theta_{\varepsilon} * \Phi^{\nu}\right)\right)^{\nu}=(-1)^{|\alpha|}\left(\theta_{\varepsilon} * \partial^{\alpha}\left(\Phi^{\nu}\right)\right)^{\nu}=(-1)^{|\alpha|}(-1)^{|\alpha|}\left(\theta_{\varepsilon} *\left(\partial^{\alpha} \Phi\right)^{\nu}\right)^{\nu}$, ou seja

$$
\partial^{\alpha}\left[\left(\theta_{\varepsilon} * \Phi^{\nu}\right)^{\nu}\right]=\left[\theta_{\varepsilon} *\left(\partial^{\alpha} \Phi\right)^{\nu}\right]^{\nu}, \quad \forall \varepsilon \in \mathbf{I} .
$$


De (2.52) e (2.53) temos que

$$
\partial^{\alpha}\left(\left(\theta_{\varepsilon} * \Phi^{\nu}\right)^{\nu}\right) \stackrel{u}{\longrightarrow} \partial^{\alpha} \Phi, \text { quando } \varepsilon \downarrow 0, \quad \forall \alpha \in \mathbb{N}^{n},
$$

e assim

$$
\left.\partial^{\alpha} g_{\varepsilon} \stackrel{u}{\longrightarrow} \partial^{\alpha} \Phi\right|_{\Omega}=\varphi, \text { quando } \varepsilon \downarrow 0, \quad \forall \alpha \in \mathbb{N}^{n} .
$$

Finalmente, de $(2.44)$ e $(2.54)$ tem-se que $g_{\varepsilon} \rightarrow \varphi$ em $\mathscr{C}_{c}^{\infty}(\Omega ; \mathbb{R})$, quando $\varepsilon \downarrow 0$, o que prova (2.46).

A Proposição 2.2.7, a seguir, nos mostrará que, dada uma $T \in \mathcal{D}^{\prime}(\Omega ; \mathbb{R})$, tem-se que $\left(\left.\left(\psi_{\varepsilon} T * \theta_{\varepsilon}\right)\right|_{\Omega}\right)_{\varepsilon} \in \mathcal{E}_{M}[\Omega ; \mathbb{R}]$

Proposição 2.2.7 Sejam $\Omega$ um aberto de $\mathbb{R}^{n}$ e $T \in \mathcal{D}^{\prime}(\Omega ; \mathbb{R})$. Tem-se que

$$
\left(\left.\left(\psi_{\varepsilon} T * \theta_{\varepsilon}\right)\right|_{\Omega}\right)_{\varepsilon} \in \mathcal{E}_{M}[\Omega ; \mathbb{R}] .
$$

Demonstração: Sejam $K \subset \subset \Omega$ e $\beta \in \mathbb{N}^{n}$. Considere $L$ aberto de $\mathbb{R}^{n}$ tal que

$$
K \subset L \subset \bar{L} \subset \subset \Omega
$$

Da Observação 2.2.4 (4), considere $\eta_{1} \in \mathbf{I}$ tal que

$$
\begin{gathered}
K \subset L \subset K_{\varepsilon}, \quad \forall \varepsilon \in \mathbf{I}_{\eta_{1}} ; \\
\operatorname{supp}^{\beta} \theta_{\varepsilon}(x-\bullet) \subset L, \quad \forall(\varepsilon, x) \in \mathbf{I}_{\eta_{1}} \times K .
\end{gathered}
$$

Fixemos $(\varepsilon, x) \in \mathbf{I}_{\eta_{1}} \times K$. Então, por $(2.12)$,

$$
\begin{aligned}
\partial^{\beta}\left(\psi_{\varepsilon} T * \theta_{\varepsilon}\right)(x) & =\left(\left(\psi_{\varepsilon} T\right) * \partial^{\beta} \theta_{\varepsilon}\right)(x) \\
& =\left(\psi_{\varepsilon} T\right)\left(\partial^{\beta} \theta_{\varepsilon}(x-\bullet)\right) \\
& =T\left(\left.\psi_{\varepsilon}(\bullet) \partial^{\beta} \theta_{\varepsilon}(x-\bullet)\right|_{\Omega}\right)
\end{aligned}
$$


e assim, de (2.56), de $\psi_{\varepsilon} \equiv 1$ em $K_{\varepsilon}$ e de (2.55), temos que

$$
\partial^{\beta}\left(\psi_{\varepsilon} T * \theta_{\varepsilon}\right)(x)=\left.T\right|_{L}\left(\left.\psi_{\varepsilon}(\bullet) \partial^{\beta} \theta_{\varepsilon}(x-\bullet)\right|_{\Omega}\right)=\left.T\right|_{L}\left(\left.\partial^{\beta} \theta_{\varepsilon}(x-\bullet)\right|_{\Omega}\right) .
$$

De $\bar{L} \subset \subset \Omega$ e de (2.13), existem $\alpha \in \mathbb{N}^{n}, W$ aberto de $\Omega$ e $f \in \mathscr{C}_{c}\left(\mathbb{R}^{n} ; \mathbb{R}\right)$ tais que

$$
\bar{L} \subset W \quad, \quad \operatorname{supp} f \subset W \text { e }\left.T\right|_{L}=\partial^{\alpha} d_{f}
$$

onde $d_{f}$ é a distribuição sobre $\Omega$ definida em (2.6). Então, por (2.12), tem-se

$$
\begin{aligned}
\partial^{\beta}\left(\psi_{\varepsilon} T * \theta_{\varepsilon}\right)(x) & =\left.T\right|_{L}\left(\left.\partial^{\beta} \theta_{\varepsilon}(x-\bullet)\right|_{\Omega}\right) \\
& =\partial^{\alpha} d_{f}\left(\left.\partial^{\beta} \theta_{\varepsilon}(x-\bullet)\right|_{\Omega}\right) \\
& =(-1)^{|\alpha|} d_{f}\left(\partial^{\alpha}\left(\left.\partial^{\beta} \theta_{\varepsilon}(x-\bullet)\right|_{\Omega}\right)\right) \\
& =d_{f}\left(\left.\partial^{\alpha+\beta} \theta_{\varepsilon}(x-\bullet)\right|_{\Omega}\right) \\
& =\int_{\Omega} \partial^{\alpha+\beta} \theta_{\varepsilon}(x-y) f(y) d y \\
& =\int_{L} \partial^{\alpha+\beta} \theta_{\varepsilon}(x-y) f(y) d y
\end{aligned}
$$

para todo $(\varepsilon, x) \in \mathbf{I}_{\eta_{1}} \times K$.

Da Proposição 2.2.1 (4.), usando que $\{x-y \mid x \in K$ e $y \in L\} \subset K+\overline{\{-y \mid y \in L\}} \subset \subset \mathbb{R}^{n}$, existem $N \in \mathbb{N}, c_{1}>0$ e $\eta \in \mathbf{I} \operatorname{com} \eta<\eta_{1}$ tais que

$$
\left|\partial^{\alpha+\beta} \theta_{\varepsilon}(x-y)\right| \leq c_{1} \varepsilon^{-N}, \quad \forall(\varepsilon, x, y) \in \mathbf{I}_{\eta} \times K \times L .
$$

Logo, temos que

$$
\begin{aligned}
\left|\partial^{\beta}\left(\psi_{\varepsilon} T * \theta_{\varepsilon}\right)(x)\right| & =\left|\int_{L} \partial^{\alpha+\beta} \theta_{\varepsilon}(x-y) f(y) d y\right| \\
& \leq \int_{L}\left|\partial^{\alpha+\beta} \theta_{\varepsilon}(x-y)\right||f(y)| d y \\
& \leq c_{1} \varepsilon^{-N} \int_{L}|f(y)| d y \\
& \leq c_{1} \varepsilon^{-N} \int_{R^{n}}|f(y)| d y,
\end{aligned}
$$

para todo $(\varepsilon, x) \in I_{\eta} \times K$. 
Seja $c:=c_{1} \int_{R^{n}}|f(y)| d y$. Então

$$
\left|\partial^{\beta}\left(\psi_{\varepsilon} T * \theta_{\varepsilon}\right)(x)\right| \leq c \varepsilon^{-N}, \quad \forall(\varepsilon, x) \in \mathbf{I}_{\eta} \times K
$$

Portanto, $\left(\left.\left(\psi_{\varepsilon} T * \theta_{\varepsilon}\right)\right|_{\Omega}\right)_{\varepsilon} \in \mathcal{E}_{M}[\Omega ; \mathbb{R}]$.

Da Proposição 2.2.7, podemos definir a aplicação

$$
T \in \mathcal{D}^{\prime}(\Omega ; \mathbb{R}) \longmapsto\left(\left.\left(\psi_{\varepsilon} T * \theta_{\varepsilon}\right)\right|_{\Omega}\right)_{\varepsilon}+\mathcal{N}[\Omega ; \mathbb{R}] .
$$

A seguir, provaremos que essa aplicação é injetora. Com esse resultado teremos construído uma imersão de $\mathcal{D}^{\prime}(\Omega ; \mathbb{R})$ em $\mathcal{G}(\Omega ; \mathbb{R})$.

Proposição 2.2.8 Sejam $\Omega$ um aberto de $\mathbb{R}^{n} \quad$ e $\quad T \in \mathcal{D}^{\prime}(\Omega ; \mathbb{R})$ tal que $\left(\left.\left(\psi_{\varepsilon} T * \theta_{\varepsilon}\right)\right|_{\Omega}\right)_{\varepsilon} \in \mathcal{N}[\Omega ; \mathbb{R}]$. Então $T \equiv 0$.

Demonstração: Seja $\varphi \in \mathscr{C}_{c}^{\infty}(\Omega ; \mathbb{R})$. Provaremos que $T(\varphi)=0$.

Da Proposição 2.2.6 temos que

$$
d_{\psi_{\varepsilon} T * \theta_{\varepsilon}} \longrightarrow T \text { em } \mathcal{D}^{\prime}(\Omega ; \mathbb{R}), \text { quando } \varepsilon \downarrow 0
$$

e, com isto,

$$
d_{\psi_{\varepsilon} T * \theta_{\varepsilon}}(\varphi) \longrightarrow T(\varphi), \text { quando } \varepsilon \downarrow 0,
$$

ou seja,

$$
\int_{\Omega} \varphi(y)\left(\psi_{\varepsilon} T * \theta_{\varepsilon}\right)(y) d y \longrightarrow T(\varphi), \text { quando } \varepsilon \downarrow 0 .
$$

Por outro lado, como $\left(\psi_{\varepsilon} T * \theta_{\varepsilon}\right)_{\varepsilon} \in \mathcal{N}[\Omega ; \mathbb{R}]$ e $\operatorname{supp} \varphi \subset \subset \Omega$, existem $c>0$ e $\eta_{1} \in \mathbf{I}$ tais que

$$
\left|\left(\psi_{\varepsilon} T * \theta_{\varepsilon}\right)(x)\right| \leq c \varepsilon, \quad \forall(\varepsilon, x) \in \mathbf{I}_{\eta_{1}} \times \operatorname{supp} \varphi
$$


Logo,

$$
\begin{aligned}
\left|\int_{\Omega} \varphi(y)\left(\psi_{\varepsilon} T * \theta_{\varepsilon}\right)(y) d y\right| & =\left|\int_{\text {supp }} \varphi(y)\left(\psi_{\varepsilon} T * \theta_{\varepsilon}\right)(y) d y\right| \\
& \leq \int_{\text {supp }}|\varphi(y)|\left|\left(\psi_{\varepsilon} T * \theta_{\varepsilon}\right)(y)\right| d y \\
& \leq c \varepsilon \int_{\Omega}|\varphi(y)| d y
\end{aligned}
$$

para todo $\varepsilon \in \mathbf{I}_{\eta_{1}}$. Portanto

$$
\left|\int_{\Omega} \varphi(y)\left(\psi_{\varepsilon} T * \theta_{\varepsilon}\right)(y) d y\right| \longrightarrow 0, \text { quando } \varepsilon \downarrow 0 .
$$

De (2.57) e (2.58) concluímos que $T(\varphi)=0$.

Como $\varphi \in \mathscr{C}_{c}^{\infty}(\Omega ; \mathbb{R})$ é arbitrária, temos que $T \equiv 0$.

Com os resultados até aqui apresentados, podemos definir a aplicação

$$
i_{\mathcal{D}^{\prime}}: T \in \mathcal{D}^{\prime}(\Omega ; \mathbb{R}) \longmapsto\left(\left.\left(\psi_{\varepsilon} T * \theta_{\varepsilon}\right)\right|_{\Omega}\right)_{\varepsilon}+\mathcal{N}[\Omega ; \mathbb{R}] \in \mathcal{G}(\Omega ; \mathbb{R})
$$

e temos que essa aplicação é injetora. No Teorema 2.2.9 iremos verificar que $i_{\mathcal{D}^{\prime}}\left(d_{f}\right)=i_{\mathscr{C} \infty(\Omega ; R)}(f)$ para toda $f \in \mathscr{C}^{\infty}(\Omega ; \mathbb{R})$, onde $i_{\mathscr{C} \infty(\Omega ; R)}$ é como em (1.1). Visto que, por (2.9), o conjunto $\left\{d_{f} \mid f \in \mathscr{C}^{\infty}(\Omega ; \mathbb{R})\right\}$ é identificado com $\mathscr{C}^{\infty}(\Omega ; \mathbb{R})$, iremos escrever $\left.\quad i_{\mathcal{D}^{\prime}}\right|_{\mathscr{C} \infty(\Omega ; R)}$ ao invés de $\left.i_{\mathcal{D}^{\prime}}\right|_{\left\{d_{f} \mid f \in \mathscr{C} \infty(\Omega ; R)\right\}}$.

Teorema 2.2.9 Seja $\Omega$ um aberto de $\mathbb{R}^{n}$. A aplicação

$$
\begin{aligned}
i_{\mathcal{D}^{\prime}}: \mathcal{D}^{\prime}(\Omega ; \mathbb{R}) & \longrightarrow \mathcal{G}(\Omega ; \mathbb{R}) \\
T & \longmapsto\left(\left.\left(\psi_{\varepsilon} T * \theta_{\varepsilon}\right)\right|_{\Omega}\right)_{\varepsilon}+\mathcal{N}[\Omega ; \mathbb{R}]
\end{aligned}
$$

é um homomorfismo injetivo de álgebras. Além disso, $\left.i_{\mathcal{D}^{\prime}}\right|_{\mathscr{C} \infty(\Omega ; R)}=i_{\mathscr{C} \infty(\Omega ; R)}$.

Demonstração: Como $i_{\mathcal{D}^{\prime}}$ é uma aplicação linear e, pela Proposição 2.2.8, é injetora, basta verificar, para concluir a prova, que $\left.i_{\mathcal{D}^{\prime}}\right|_{\mathscr{C} \infty(\Omega ; R)}=i_{\mathscr{C} \infty(\Omega ; R)}$. 
Para facilitar a escrita escreveremos $i_{\mathscr{C} \infty}$ ao invés de $i_{\mathscr{C} \infty(\Omega ; R)}$.

Seja $f \in \mathscr{C}^{\infty}(\Omega ; \mathbb{R})$ e considere $\Delta:=i_{\mathcal{D}^{\prime}}\left(d_{f}\right)-i_{\mathscr{C} \infty}(f)$, onde $d_{f}$ é a distribuição sobre $\Omega$ definida em (2.6). Um representante de $\Delta$ é dado por

$$
\Delta_{\varepsilon}(x):=\left(\psi_{\varepsilon} d_{f} * \theta_{\varepsilon}\right)(x)-f(x), \quad \forall(\varepsilon, x) \in \mathbf{I} \times \Omega .
$$

Provaremos que $\left(\Delta_{\varepsilon}\right)_{\varepsilon} \in \mathcal{N}[\Omega ; \mathbb{R}]$. Para essa finalidade usaremos o Teorema de Nulidade em $\mathcal{E}_{M}[\Omega ; \mathbb{R}]$ (Teorema 1.2.5).

Sejam $K \subset \subset \Omega$ e $m \in \mathbb{N}$. Considere $L$ aberto de $\mathbb{R}^{n}$ tal que

$$
K \subset L \subset \bar{L} \subset \subset \Omega
$$

Sejam $\eta_{1} \in \mathbf{I}$ e $\nu>0$ como na Observação 2.2.4 (4.), isto é,

$$
\begin{gathered}
K \subset L \subset K_{\varepsilon}, \quad \forall \varepsilon \in \mathbf{I}_{\eta_{1}} ; \\
\operatorname{supp} \theta_{\varepsilon}(x-\bullet) \subset \overline{B_{\nu}(x)} \subset L, \quad \forall(\varepsilon, x) \in \mathbf{I}_{\eta_{1}} \times K .
\end{gathered}
$$

Logo,

$$
\begin{aligned}
\left(\psi_{\varepsilon} d_{f} * \theta_{\varepsilon}\right)(x) & =\left(\psi_{\varepsilon} d_{f}\right)\left(\theta_{\varepsilon}(x-\bullet)\right) \\
& =d_{f}\left(\left.\psi_{\varepsilon}(\bullet) \theta_{\varepsilon}(x-\bullet)\right|_{\Omega}\right) \\
& =\left.d_{f}\right|_{L}\left(\left.\psi_{\varepsilon}(\bullet) \theta_{\varepsilon}(x-\bullet)\right|_{\Omega}\right) \\
& =\left.d_{f}\right|_{L}\left(\left.\theta_{\varepsilon}(x-\bullet)\right|_{\Omega}\right)
\end{aligned}
$$

para todo $(\varepsilon, x) \in \mathbf{I}_{\eta_{1}} \times K$, onde a terceira igualdade decorre de (2.60) e a última, do fato de que $\psi_{\varepsilon} \equiv 1$ em $K_{\varepsilon}$ e de $(2.59)$.

Da Proposição 2.2 .3 (3.), existe $\left(n_{\varepsilon}\right)_{\varepsilon} \in \mathcal{N}(\mathbb{R})$ tal que

$$
\int_{R^{n}} \theta_{\varepsilon}(y) d y=1+n_{\varepsilon}, \quad \forall \varepsilon \in \mathbf{I}
$$

De (2.60) e (2.61) temos, para $(\varepsilon, x) \in \mathbf{I}_{\eta_{1}} \times K$, que

$$
1+n_{\varepsilon}=\int_{R^{n}} \theta_{\varepsilon}(y) d y=\int_{R^{n}} \theta_{\varepsilon}(x-y) d y=\int_{L} \theta_{\varepsilon}(x-y) d y .
$$


Assim,

$$
\begin{aligned}
\Delta_{\varepsilon}(x) & =\left(\psi_{\varepsilon} d_{f} * \theta_{\varepsilon}\right)(x)-f(x) \\
& =\left.d_{f}\right|_{L}\left(\left.\theta_{\varepsilon}(x-\bullet)\right|_{\Omega}\right)-f(x) \\
& =d_{f}\left(\left.\theta_{\varepsilon}(x-\bullet)\right|_{\Omega}\right)-f(x) \\
& =\int_{\Omega} f(y) \theta_{\varepsilon}(x-y) d y-f(x) \\
& =\int_{L} f(y) \theta_{\varepsilon}(x-y) d y-f(x)\left[\int_{L} \theta_{\varepsilon}(x-y) d y-n_{\varepsilon}\right] \\
& =\int_{L}[f(y)-f(x)] \theta_{\varepsilon}(x-y) d y+f(x) n_{\varepsilon} \\
& =\int_{B_{\nu}(x)}[f(y)-f(x)] \theta_{\varepsilon}(x-y) d y+f(x) n_{\varepsilon},
\end{aligned}
$$

para $(\varepsilon, x) \in \mathbf{I}_{\eta_{1}} \times K$ onde a última igualdade decorre de (2.60).

Como $f \in \mathscr{C}^{\infty}(\Omega ; \mathbb{R})$ podemos utilizar o polinômio de Taylor de ordem $m+1 \mathrm{em}$ torno de $x \in K$. Para facilitar a escrita desse polinômio denotaremos, para $s \in \mathbb{N} \backslash\{0\}$, $v=\left(v_{1}, \cdots, v_{n}\right) \in \mathbb{R}^{n}$ e $a=\left(a_{1}, \cdots, a_{n}\right) \in \mathbb{R}^{n}$,

$$
\nabla^{s} f(a) \bullet v^{s}:=\sum_{i_{1}=1}^{n} \sum_{i_{2}=1}^{n} \cdots \sum_{i_{s}=1}^{n} \frac{\partial^{s} f}{\partial x_{i_{1}} \partial x_{i_{2}} \cdots \partial x_{i_{s}}}(a) v_{i_{1}} v_{i_{2}} \cdots v_{i_{s}}
$$

Portanto, para $y \in \overline{B_{\nu}(x)} \subset L$, existe $\sigma_{y} \in[0,1]$ tal que

$$
f(y)-f(x)=\sum_{i=1}^{m+1} \frac{1}{i !} \nabla^{i} f(x) \bullet(y-x)^{i}+\frac{1}{(m+2) !} \nabla^{m+2} f\left(x+\sigma_{y}(x-y)\right) \bullet(y-x)^{m+2} .
$$

Logo,

$$
\begin{aligned}
\Delta_{\varepsilon}(x)= & \int_{\frac{B_{\nu}(x)}{B}}[f(y)-f(x)] \theta_{\varepsilon}(x-y) d y+f(x) n_{\varepsilon} \\
= & \sum_{i=1}^{m+1} \frac{\int}{B_{\nu}(x)} \frac{1}{i !} \nabla^{i} f(x) \bullet(y-x)^{i} \theta_{\varepsilon}(x-y) d y+ \\
& +\int_{\frac{1}{B_{\nu}(x)}} \frac{1}{(m+2) !} \nabla^{m+2} f\left(x+\sigma_{y}(x-y)\right) \bullet(y-x)^{m+2} \theta_{\varepsilon}(x-y) d y+f(x) n_{\varepsilon},
\end{aligned}
$$

$\operatorname{para}(\varepsilon, x) \in \mathbf{I}_{\eta_{1}} \times K$. 
De $f \in \mathscr{C}^{\infty}(\Omega ; \mathbb{R})$, existe $M>0$ tal que

$$
\left|\partial^{\alpha} f(w)\right| \leq M, \quad \forall w \in \bar{L}, \quad \forall \alpha \in \mathbb{N}^{n} \operatorname{com}|\alpha| \leq m+2,
$$

e de $\left(n_{\varepsilon}\right)_{\varepsilon} \in \mathcal{N}(\mathbb{R})$ e $\left(\int_{R^{n}} w^{\beta} \theta_{\varepsilon}(w) d w\right)_{\varepsilon} \in \mathcal{N}(\mathbb{R})$, para todo $\beta \in \mathbb{N}^{n} \backslash\{0\}$ (Proposição 2.2 .3 (4.)), existem $c_{1}>0$ e $\eta \in \mathbf{I} \operatorname{com} \eta<\eta_{1}$ tais que

$$
\begin{gathered}
\left|n_{\varepsilon}\right| \leq c_{1} \varepsilon^{m}, \quad \forall \varepsilon \in \mathbf{I}_{\eta} ; \\
\left|\int_{R^{n}} w^{\beta} \theta_{\varepsilon}(w) d w\right| \leq c_{1} \varepsilon^{m}, \quad \forall \varepsilon \in \mathbf{I}_{\eta} \quad \text { e } \forall \beta \in \mathbb{N}^{n} \backslash\{0\} \quad \operatorname{com}|\beta| \leq m+2 .
\end{gathered}
$$

Fixemos $(\varepsilon, x) \in \mathbf{I}_{\eta} \times K$ e sejam

$$
\begin{gathered}
G_{\varepsilon}(x):=\sum_{i=1}^{m+1} \frac{\int}{B_{\nu}(x)} \frac{1}{i !} \nabla^{i} f(x) \bullet(x-y)^{i} \theta_{\varepsilon}(x-y) d y \\
H_{\varepsilon}(x):=\int_{\frac{B_{\nu}(x)}{(m+2) !}} \frac{1}{(m+2} f\left(x+\sigma_{y}(x-y)\right) \bullet(y-x)^{m+2} \theta_{\varepsilon}(x-y) d y .
\end{gathered}
$$

Então,

$$
\left|\Delta_{\varepsilon}(x)\right| \leq\left|G_{\varepsilon}(x)\right|+\left|H_{\varepsilon}(x)\right|+|f(x)|\left|n_{\varepsilon}\right| .
$$

Notemos que

$$
\begin{aligned}
G_{\varepsilon}(x) & =\sum_{i=1}^{m+1} \frac{1}{i !} \frac{\int}{B_{\nu}(x)} \sum_{|\beta|=i} \partial^{\beta} f(x)(x-y)^{\beta} \theta_{\varepsilon}(x-y) d y \\
& =\sum_{i=1}^{m+1} \sum_{|\beta|=i} \frac{1}{i !} \partial^{\beta} f(x) \int_{\frac{B_{\nu}(x)}{}}(x-y)^{\beta} \theta_{\varepsilon}(x-y) d y \\
& =\sum_{i=1}^{m+1} \sum_{|\beta|=i} \frac{1}{i !} \partial^{\beta} f(x) \int_{R^{n}}(x-y)^{\beta} \theta_{\varepsilon}(x-y) d y \\
& =\sum_{i=1}^{m+1} \sum_{|\beta|=i} \frac{1}{i !} \partial^{\beta} f(x) \int_{R^{n}} w^{\beta} \theta_{\varepsilon}(w) d w,
\end{aligned}
$$

onde a penúltima igualdade decorre de (2.60). Logo, de (2.63) e (2.65) temos

$$
\left|G_{\varepsilon}(x)\right| \leq\left(\sum_{i=1}^{m+1} \sum_{|\beta|=i} \frac{M}{i !} c_{1}\right) \varepsilon^{m} .
$$


Como

$$
\begin{aligned}
H_{\varepsilon}(x) & =\int_{\frac{1}{B_{\nu}(x)}} \frac{1}{(m+2) !} \sum_{|\beta|=m+2} \partial^{\beta} f\left(x+\sigma_{y}(x-y)\right)(y-x)^{\beta} \theta_{\varepsilon}(x-y) d y \\
& =\sum_{|\beta|=m+2} \frac{1}{(m+2) !} \int_{\frac{B_{\nu}(x)}{2}} \partial^{\beta} f\left(x+\sigma_{y}(x-y)\right)(y-x)^{\beta} \frac{1}{\varepsilon^{n}} \rho\left(\frac{x-y}{\varepsilon}\right) \chi\left(\frac{x-y}{\sqrt{\varepsilon}}\right) d y,
\end{aligned}
$$

temos, por $(2.63)$ e de $0 \leq \chi \leq 1$, que

$$
\begin{aligned}
\left|H_{\varepsilon}(x)\right| & \leq \sum_{|\beta|=m+2} \frac{1}{(m+2) !} \int_{\frac{B_{\nu}(x)}{(m \mid=m+2}}\left|M(x-y)^{\beta} \frac{1}{\varepsilon^{n}} \rho\left(\frac{x-y}{\varepsilon}\right)\right| d y \\
& \leq \sum_{|\beta|} \frac{1}{(m+2) !} M \int_{\frac{B_{\frac{\nu}{\varepsilon}}(0)}{}}\left|\varepsilon^{|\beta|} w^{\beta} \rho(w)\right| d w \\
& \leq \sum_{|\beta|=m+2} \frac{1}{(m+2) !} M \varepsilon^{m+2} \int\left|w^{\beta} \rho(w)\right| d w .
\end{aligned}
$$

Logo, pela Observação 2.1.14 (3.), temos que

$$
\left|H_{\varepsilon}(x)\right| \leq\left(\sum_{|\beta|=m+2} \frac{M}{(m+2) !} \int_{R^{n}}\left|w^{\beta} \rho(w)\right| d w\right) \varepsilon^{m} .
$$

De (2.63), (2.64), (2.67) e (2.68) concluímos que

$$
\left|\Delta_{\varepsilon}(x)\right| \leq\left(\sum_{i=1}^{m+1} \sum_{|\beta|=i} \frac{M c_{1}}{i !}\right) \varepsilon^{m}+\left(\sum_{|\beta|=m+2} \frac{M}{(m+2) !} \int_{R^{n}}\left|w^{\beta} \rho(w)\right| d w\right) \varepsilon^{m}+M c_{1} \varepsilon^{m} .
$$

Portanto, se $c:=\sum_{i=1}^{m+1} \sum_{|\beta|=i} \frac{M c_{1}}{i !}+\sum_{|\beta|=m+2} \frac{M}{(m+2) !} \int_{R^{n}}\left|w^{\beta} \rho(w)\right| d w+M c_{1}$, temos

$$
\left|\Delta_{\varepsilon}(x)\right| \leq c \varepsilon^{m}, \quad \forall(\varepsilon, x) \in \mathbf{I}_{\eta} \times K
$$

Assim, pelo Teorema de Nulidade em $\mathcal{E}_{M}[\Omega ; \mathbb{R}]$ (Teorema 1.2.5), concluímos que $\left(\Delta_{\varepsilon}\right)_{\varepsilon} \in \mathcal{N}[\Omega ; \mathbb{R}]$.

No caso em que $\Omega=\mathbb{R}^{n}$, a aplicação $i_{\mathcal{D}^{\prime}}$, como observaremos a seguir, tem uma expressão mais simples. 
Observação 2.2.10 Se $\Omega=\mathbb{R}^{n}$, então a aplicação $i_{\mathcal{D}^{\prime}}$, definida no Teorema 2.2.9, é a aplicação

$$
\begin{aligned}
\mathcal{D}^{\prime}\left(\mathbb{R}^{n} ; \mathbb{R}\right) & \longrightarrow \mathcal{G}\left(\mathbb{R}^{n} ; \mathbb{R}\right) \\
T & \longmapsto\left(T * \theta_{\varepsilon}\right)_{\varepsilon}+\mathcal{N}\left[\mathbb{R}^{n} ; \mathbb{R}\right]
\end{aligned}
$$

De fato, basta observar que, para todo $r>0$, tem-se, pela Observação 2.2 .4 (4.), que existe $\eta \in \mathbf{I}$ tal que

$$
B_{r+2}(0) \subset K_{\varepsilon} \quad \text { e } \operatorname{supp} \theta_{\varepsilon}(x-\bullet) \subset B_{r+2}(0), \quad \forall(\varepsilon, x) \in \mathbf{I}_{\eta} \times \overline{B_{r}(0)}
$$

Assim,

$$
\begin{aligned}
\left(T * \theta_{\varepsilon}\right)(x) & =T\left(\theta_{\varepsilon}(x-\bullet)\right) \\
& =\left.T\right|_{B_{r+2}(0)}\left(\theta_{\varepsilon}(x-\bullet)\right) \\
& =\left.T\right|_{B_{r+2}(0)}\left(\psi_{\varepsilon}(\bullet) \theta_{\varepsilon}(x-\bullet)\right) \\
& =T\left(\psi_{\varepsilon}(\bullet) \theta_{\varepsilon}(x-\bullet)\right) \\
& =\left(\left(\psi_{\varepsilon} T\right) * \theta_{\varepsilon}\right)(x)
\end{aligned}
$$

para todo $(\varepsilon, x) \in \mathbf{I}_{\eta} \times \overline{B_{r}(0)}$, onde a terceira igualdade segue de $\psi_{\varepsilon} \equiv 1 \mathrm{em} K_{\varepsilon}$.

Exemplo 2.2.11 1. Sejam $\Omega=\mathbb{R}^{n}, a \in \mathbb{R}^{n}$ e $\delta_{a}$ a "Medida de Dirac" (Exemplo 2.1.3 (1.)). Então

$$
i_{\mathcal{D}^{\prime}}\left(\delta_{a}\right)=\left(x \in \mathbb{R}^{n} \longmapsto \rho_{\varepsilon}(x-a)\right)_{\varepsilon}+\mathcal{N}\left[\mathbb{R}^{n} ; \mathbb{R}\right]
$$

2. Sejam $a \in \mathbb{R}$ e $T_{H_{a}}$ o "Degrau de Heaviside" (Exemplo 2.1.3 (2.)). Então

$$
i_{\mathcal{D}^{\prime}}\left(T_{H_{a}}\right)=\left(x \in \mathbb{R} \longmapsto \int_{[a,+\infty[} \theta_{\varepsilon}(x-y) d y\right)_{\varepsilon}+\mathcal{N}[\mathbb{R} ; \mathbb{R}] .
$$

De fato, basta usar a Observação 2.2.10 e observar que

$$
\left(\delta_{a} * \theta_{\varepsilon}\right)(x)=\delta_{a}\left(\theta_{\varepsilon}(x-\bullet)\right)=\theta_{\varepsilon}(x-a), \quad \forall(\varepsilon, x) \in \mathbf{I} \times \mathbb{R}^{n}
$$




$$
\left(T_{H_{a}} * \theta_{\varepsilon}\right)(x)=T_{H_{a}}\left(\theta_{\varepsilon}(x-\bullet)\right)=\int_{[a,+\infty[} \theta_{\varepsilon}(x-y) d y, \quad \forall(\varepsilon, x) \in \mathbf{I} \times \mathbb{R},
$$

e que, da Proposição 2.2.3, (1.), tem-se que

$$
\left(x \in \mathbb{R}^{n} \longmapsto \theta_{\varepsilon}(x-a)-\rho_{\varepsilon}(x-a)\right)_{\varepsilon} \in \mathcal{N}\left[\mathbb{R}^{n} ; \mathbb{R}\right] .
$$

Finalizamos esta seção com o seguinte resultado.

Proposição 2.2.12 Sejam $\Omega$ um aberto de $\mathbb{R}^{n}$ e $\alpha \in \mathbb{N}^{n}$. Então

$$
i_{\mathcal{D}^{\prime}}\left(\partial^{\alpha} T\right)=\partial^{\alpha}\left(i_{\mathcal{D}^{\prime}}(T)\right), \quad \forall T \in \mathcal{D}^{\prime}(\Omega ; \mathbb{R}) .
$$

Demonstração: Notemos que

$$
\begin{gathered}
i_{\mathcal{D}^{\prime}}\left(\partial^{\alpha} T\right)=\left(x \in \Omega \longmapsto\left(\psi_{\varepsilon} \partial^{\alpha} T * \theta_{\varepsilon}\right)(x)=\partial^{\alpha} T\left(\left.\psi_{\varepsilon}(\bullet) \theta_{\varepsilon}(x-\bullet)\right|_{\Omega}\right)\right)_{\varepsilon}+\mathcal{N}[\Omega ; \mathbb{R}] ; \\
i_{\mathcal{D}^{\prime}}(T)=\left(x \in \Omega \longmapsto\left(\psi_{\varepsilon} T * \theta_{\varepsilon}\right)(x)\right)_{\varepsilon}+\mathcal{N}[\Omega ; \mathbb{R}] .
\end{gathered}
$$

Seja $K \subset \subset \Omega$. Considere $L$ aberto de $\mathbb{R}^{n}$ tal que

$$
K \subset L \subset \bar{L} \subset \subset \Omega
$$

Seja $\eta_{1} \in \mathbf{I}$ como na Observação 2.2 .4 (4.), isto é,

$$
\begin{gathered}
K \subset L \subset K_{\varepsilon}, \quad \forall \varepsilon \in \mathbf{I}_{\eta_{1}} ; \\
\operatorname{supp}_{\varepsilon}(x-\bullet) \subset L, \quad \forall(\varepsilon, x) \in \mathbf{I}_{\eta_{1}} \times K .
\end{gathered}
$$

De (2.69), (2.70), de $\psi_{\varepsilon} \equiv 1$ em $K_{\varepsilon}$ e de (2.12), temos, para todo $(\varepsilon, x) \in \mathbf{I}_{\eta_{1}} \times K$, que

$$
\begin{aligned}
\partial^{\alpha} T\left(\left.\psi_{\varepsilon}(\bullet) \theta_{\varepsilon}(x-\bullet)\right|_{\Omega}\right) & =\left.\partial^{\alpha} T\right|_{L}\left(\left.\theta_{\varepsilon}(x-\bullet)\right|_{\Omega}\right) \\
& =\partial^{\alpha} T\left(\left.\theta_{\varepsilon}(x-\bullet)\right|_{\Omega}\right) \\
& =(-1)^{|\alpha|} T\left(\partial^{\alpha}\left(\left.\theta_{\varepsilon}(x-\bullet)\right|_{\Omega}\right)\right)
\end{aligned}
$$




$$
\begin{aligned}
& =T\left(\left.\partial^{\alpha} \theta_{\varepsilon}(x-\bullet)\right|_{\Omega}\right) \\
& =\left.T\right|_{L}\left(\left.\partial^{\alpha} \theta_{\varepsilon}(x-\bullet)\right|_{\Omega}\right) \\
& =\left.T\right|_{L}\left(\left.\psi_{\varepsilon}(\bullet) \partial^{\alpha} \theta_{\varepsilon}(x-\bullet)\right|_{\Omega}\right) \\
& =T\left(\left.\psi_{\varepsilon}(\bullet) \partial^{\alpha} \theta_{\varepsilon}(x-\bullet)\right|_{\Omega}\right) \\
& =\left(\psi_{\varepsilon} T * \partial^{\alpha} \theta_{\varepsilon}\right)(x) \\
& =\partial^{\alpha}\left(\left(\psi_{\varepsilon} T * \theta_{\varepsilon}\right)\right)(x) .
\end{aligned}
$$

Logo, usando o Teorema de Nulidade em $\mathcal{E}_{M}[\Omega ; \mathbb{R}]$ (Teorema 1.2.5), temos que

$$
\left(x \in \mathbb{R}^{n} \longmapsto \partial^{\alpha} T\left(\left.\psi_{\varepsilon}(\bullet) \theta_{\varepsilon}(x-\bullet)\right|_{\Omega}\right)-\partial^{\alpha}\left(\psi_{\varepsilon} T * \theta_{\varepsilon}\right)(x)\right)_{\varepsilon}
$$

é nula, ou seja, $i_{\mathcal{D}^{\prime}}\left(\partial^{\alpha} T\right)=\partial^{\alpha} i_{\mathcal{D}^{\prime}}(T)$.

\subsection{Uma imersão de $\mathcal{S}^{\prime}\left(\mathbb{R}^{n} ; \mathbb{R}\right)$ em $\mathcal{G}_{\tau}\left(\mathbb{R}^{n} ; \mathbb{R}\right)$}

A imersão do conjunto $\mathcal{S}^{\prime}\left(\mathbb{R}^{n} ; \mathbb{R}\right)$ em $\mathcal{G}_{\tau}\left(\mathbb{R}^{n} ; \mathbb{R}\right)$ aqui apresentada foi baseada na encontrada em [GKOS] e na Observação 2.2.10. Antes de construirmos tal imersão lembramos que, em toda esta seção, os símbolos $\rho_{\varepsilon}$ e $\theta_{\varepsilon}$ denotarão as funções definidas em (2.24) e $(2.25)$.

Iniciaremos mostrando, como a partir de uma distribuição temperada pode-se construir uma função moderada temperada.

Proposição 2.3.1 Seja $T \in \mathcal{S}^{\prime}\left(\mathbb{R}^{n} ; \mathbb{R}\right)$. Então $\left(T * \theta_{\varepsilon}\right)_{\varepsilon} \in \mathcal{E}_{\tau}\left[\mathbb{R}^{n} ; \mathbb{R}\right]$.

Demonstração: Como $T \in \mathcal{S}^{\prime}\left(\mathbb{R}^{n} ; \mathbb{R}\right)$ temos, por $(2.22)$, que existem $f \in \mathscr{C}\left(\mathbb{R}^{n} ; \mathbb{R}\right)$ limitada, $N_{1} \in \mathbb{N}$ e $\alpha \in \mathbb{N}^{n}$ tais que

$$
T=\partial^{\alpha} d_{(1+|y|)^{N_{1}} f} .
$$


Sejam $\varepsilon \in \mathbf{I}$ e $\beta \in \mathbb{N}^{n}$. Então, por (2.21), temos que

$$
\begin{aligned}
\partial^{\beta}\left(T * \theta_{\varepsilon}\right)(x) & =\left(T * \partial^{\beta} \theta_{\varepsilon}\right)(x) \\
& =\left(\partial^{\alpha} d_{(1+|y|)^{N_{1}} f} * \partial^{\beta} \theta_{\varepsilon}\right)(x) \\
& =\partial^{\alpha} d_{(1+|y|)^{N_{1}} f}\left(\partial^{\beta} \theta_{\varepsilon}(x-\bullet)\right) \\
& =(-1)^{|\alpha|} d_{(1+|y|)^{N_{1}} f}\left(\partial^{\alpha}\left(\partial^{\beta} \theta_{\varepsilon}(x-\bullet)\right)\right) \\
& =d_{(1+|y|)^{N_{1}} f}\left(\partial^{\alpha+\beta} \theta_{\varepsilon}(x-\bullet)\right) \\
& =\int_{R^{n}}(1+|y|)^{N_{1}} f(y) \partial^{\alpha+\beta} \theta_{\varepsilon}(x-y) d y
\end{aligned}
$$

ou seja,

$$
\partial^{\beta}\left(T * \theta_{\varepsilon}\right)(x)=\int_{R^{n}}(1+|x-w|)^{N_{1}} f(x-w) \partial^{\alpha+\beta} \theta_{\varepsilon}(w) d w, \quad \forall x \in \mathbb{R}^{n} .
$$

Note que

$$
(1+|x-w|)^{N_{1}} \leq(1+|x|+|w|)^{N_{1}}=(1+|x|+(1+|x|)|w|)^{N_{1}}=(1+|x|)^{N_{1}}(1+|w|)^{N_{1}}
$$

para todo $(x, w) \in \mathbb{R}^{n} \times \mathbb{R}^{n}$. Logo,

$$
\left|\partial^{\beta}\left(T * \theta_{\varepsilon}\right)(x)\right| \leq \int_{R^{n}}(1+|x|)^{N_{1}}(1+|w|)^{N_{1}}|f(x-w)|\left|\partial^{\alpha+\beta} \theta_{\varepsilon}(w)\right| d w,
$$

para todo $x \in \mathbb{R}^{n}$.

Observe ainda que

$$
\begin{aligned}
\left|\partial^{\alpha+\beta} \theta_{\varepsilon}(w)\right| & =\left|\sum_{0 \leq \gamma \leq \alpha+\beta}\left(\begin{array}{c}
\alpha+\beta \\
\gamma
\end{array}\right) \partial^{\gamma} \rho\left(\frac{w}{\varepsilon}\right) \varepsilon^{-n-|\gamma|} \partial^{\alpha+\beta-\gamma} \chi\left(\frac{w}{\sqrt{\varepsilon}}\right) \varepsilon^{\frac{-|\alpha+\beta|+\gamma}{2}}\right| \\
& \leq \sum_{0 \leq \gamma \leq \alpha+\beta}\left(\begin{array}{c}
\alpha+\beta \\
\gamma
\end{array}\right)\left|\partial^{\gamma} \rho\left(\frac{w}{\varepsilon}\right) \partial^{\alpha+\beta-\gamma} \chi\left(\frac{w}{\sqrt{\varepsilon}}\right)\right| \varepsilon^{-n-|\alpha+\beta|},
\end{aligned}
$$

para todo $w \in \mathbb{R}^{n}$.

Seja, para $\gamma \in \mathbb{N}^{n}, g_{\gamma}$ a função definida em $\mathbb{R}^{n}$ por

$$
g_{\gamma}(w):=\partial^{\gamma} \rho\left(\frac{w}{\varepsilon}\right) \partial^{\alpha+\beta-\gamma} \chi\left(\frac{w}{\sqrt{\varepsilon}}\right), \quad \forall w \in \mathbb{R}^{n} .
$$




\section{Então}

$$
\left|\partial^{\beta}\left(T * \theta_{\varepsilon}\right)(x)\right| \leq(1+|x|)^{N_{1}} \sum_{0 \leq \gamma \leq \alpha+\beta}\left(\begin{array}{c}
\alpha+\beta \\
\gamma
\end{array}\right) \int_{R^{n}}(1+|w|)^{N_{1}}|f(x-w)|\left|g_{\gamma}(w)\right| \varepsilon^{-n-|\alpha+\beta|} d w,
$$

para todo $x \in \mathbb{R}^{n}$.

Do fato de

$$
\int_{R^{n}}\left|g_{\gamma}(w)\right|(1+|w|)^{N_{1}}|f(x-w)| d w=\int_{R^{n}}\left|g_{\gamma}(\varepsilon y)\right|(1+\varepsilon|y|)^{N_{1}}|f(x-\varepsilon y)| \varepsilon^{n} d y,
$$

para todo $\gamma \in \mathbb{N}^{n}$, temos que

$$
\left|\partial^{\beta}\left(T * \theta_{\varepsilon}\right)(x)\right| \leq(1+|x|)^{N_{1}} \varepsilon^{-|\alpha+\beta|} \sum_{0 \leq \gamma \leq \alpha+\beta}\left(\begin{array}{c}
\alpha+\beta \\
\gamma
\end{array}\right) \int_{R^{n}}\left|g_{\gamma}(\varepsilon y)\right|(1+\varepsilon|y|)^{N_{1}}|f(x-\varepsilon y)| d y,
$$

para todo $x \in \mathbb{R}^{n}$.

Como $\chi \in \mathscr{C}_{c}^{\infty}\left(\mathbb{R}^{n} ; \mathbb{R}\right), f \in \mathscr{C}\left(\mathbb{R}^{n} ; \mathbb{R}\right)$ é limitada e $\rho \in \mathcal{S}\left(\mathbb{R}^{n} ; \mathbb{R}\right)$, existe $M>0$ e, para todo $\gamma \in \mathbb{N}^{n}$ com $\gamma \leq \alpha+\beta$, existe $c_{\gamma}>0$ tais que

$$
\begin{gathered}
|f(z)| \leq M, \quad \forall z \in \mathbb{R}^{n} ; \\
\left|\partial^{\alpha+\beta-\gamma} \chi(z)\right| \leq c_{\gamma}, \quad \forall z \in \mathbb{R}^{n} ; \\
\left|\partial^{\gamma} \rho(z)\right| \leq c_{\gamma}(1+|z|)^{-N_{1}-2 n}, \quad \forall z \in \mathbb{R}^{n} .
\end{gathered}
$$

Logo, $\left|g_{\gamma}(\varepsilon y)\right| \leq c_{\gamma}^{2}(1+|y|)^{-N_{1}-2 n}$, para todo $y \in \mathbb{R}^{n}$.

Seja $r:=\max \left\{M, \max \left\{c_{\gamma} \mid \gamma \in \mathbb{N}^{n}\right.\right.$ e $\left.\left.\gamma \leq \alpha+\beta\right\}\right\}$. Assim,

$$
\begin{aligned}
\left|\partial^{\beta}\left(T * \theta_{\varepsilon}\right)(x)\right| & \leq(1+|x|)^{N_{1}} \varepsilon^{-|\alpha+\beta|} \sum_{0 \leq \gamma \leq \alpha+\beta}\left(\begin{array}{c}
\alpha+\beta \\
\gamma
\end{array}\right) \int_{R^{n}} r^{3}(1+|y|)^{-N_{1}-2 n}(1+\varepsilon|y|)^{N_{1}} d y \\
& \leq(1+|x|)^{N_{1}} \varepsilon^{-|\alpha+\beta|} \sum_{0 \leq \gamma \leq \alpha+\beta}\left(\begin{array}{c}
\alpha+\beta \\
\gamma
\end{array}\right) r^{3} \int_{R^{n}} \frac{1}{(1+|y|)^{2 n}} d y,
\end{aligned}
$$

para todo $x \in \mathbb{R}^{n}$.

$$
\begin{gathered}
\text { Sejam } c:=\sum_{0 \leq \gamma \leq \alpha+\beta}\left(\begin{array}{c}
\alpha+\beta \\
\gamma
\end{array}\right) r^{3} \int_{R^{n}} \frac{1}{(1+|y|)^{2 n}} d y \text { e } N:=\max \left\{N_{1},|\alpha+\beta|\right\} . \text { Então } \\
\left|\partial^{\beta}\left(T * \theta_{\varepsilon}\right)(x)\right| \leq c(1+|x|)^{N} \varepsilon^{-N}, \quad \forall x \in \mathbb{R}^{n} .
\end{gathered}
$$

Portanto $\left(T * \theta_{\varepsilon}\right)_{\varepsilon} \in \mathcal{E}_{\tau}\left[\mathbb{R}^{n} ; \mathbb{R}\right]$. 
A partir da Proposição 2.3.1, pode-se definir a aplicação linear

$$
\begin{aligned}
i_{\mathcal{S}^{\prime}}: \mathcal{S}^{\prime}\left(\mathbb{R}^{n} ; \mathbb{R}\right) & \longrightarrow \mathcal{G}_{\tau}\left(\mathbb{R}^{n} ; \mathbb{R}\right) \\
T & \longmapsto\left(T * \theta_{\varepsilon}\right)_{\varepsilon}+\mathcal{N}_{\tau}\left[\mathbb{R}^{n} ; \mathbb{R}\right]
\end{aligned}
$$

Para obtermos que essa aplicação é injetora necessitamos do seguinte resultado.

Proposição 2.3.2 Seja $T \in \mathcal{S}^{\prime}\left(\mathbb{R}^{n} ; \mathbb{R}\right)$ tal que $\left(T * \theta_{\varepsilon}\right)_{\varepsilon} \in \mathcal{N}_{\tau}\left[\mathbb{R}^{n} ; \mathbb{R}\right]$. Então $T \equiv 0$.

Demonstração: $\operatorname{De}\left(T * \theta_{\varepsilon}\right)_{\varepsilon} \in \mathcal{N}_{\tau}\left[\mathbb{R}^{n} ; \mathbb{R}\right]$ e $\mathcal{N}_{\tau}\left[\mathbb{R}^{n} ; \mathbb{R}\right] \subset \mathcal{N}\left[\mathbb{R}^{n} ; \mathbb{R}\right]$ (Proposição 1.3.12 (2.)), temos que $\left(T * \theta_{\varepsilon}\right)_{\varepsilon} \in \mathcal{N}\left[\mathbb{R}^{n} ; \mathbb{R}\right]$.

Seja $S:=\left.T\right|_{\mathscr{C}_{c}^{\infty}\left(R^{n} ; R\right)}$. Então, como foi observado após a Definição 2.1.18, a função $S$ é uma distribuição sobre $\mathbb{R}^{n}$.

Observe que, para $(\varepsilon, x) \in \mathbf{I} \times \mathbb{R}^{n}$, tem-se $\operatorname{supp} \theta_{\varepsilon}(x-\bullet) \subset \overline{B_{2}(x)} . \quad$ Logo $\theta_{\varepsilon}(x-\bullet) \in \mathscr{C}_{c}^{\infty}\left(\mathbb{R}^{n} ; \mathbb{R}\right)$, e assim

$$
\left(T * \theta_{\varepsilon}\right)_{\varepsilon}(x)=T\left(\theta_{\varepsilon}(x-\bullet)\right)=S\left(\theta_{\varepsilon}(x-\bullet)\right)=\left(S * \theta_{\varepsilon}\right)_{\varepsilon}(x), \quad \forall(\varepsilon, x) \in \mathbf{I} \times \mathbb{R}^{n} .
$$

Dos fatos acima concluímos que

$$
S \in \mathcal{D}^{\prime}\left(\mathbb{R}^{n} ; \mathbb{R}\right) \quad \text { e } \quad\left(S * \theta_{\varepsilon}\right)_{\varepsilon} \in \mathcal{N}\left[\mathbb{R}^{n} ; \mathbb{R}\right]
$$

Dessa forma, do Teorema 2.2.9 e da Observação 2.2.10, obtemos que $S \equiv 0$, ou seja, $\left.T\right|_{\mathscr{C}_{c}^{\infty}\left(R^{n} ; R\right)} \equiv 0$.

Como $\mathscr{C}_{c}^{\infty}\left(\mathbb{R}^{n} ; \mathbb{R}\right)$ é denso em $\mathcal{S}\left(\mathbb{R}^{n} ; \mathbb{R}\right)([\mathrm{Cor}])$ e $\left.T\right|_{\mathscr{C}_{c}^{\infty}\left(R^{n} ; R\right)} \equiv 0$, segue $T \equiv 0$.

Com os resultados até aqui apresentados, podemos definir a aplicação

$$
i_{\mathcal{S}^{\prime}}: T \in \mathcal{S}^{\prime}\left(\mathbb{R}^{n} ; \mathbb{R}\right) \longmapsto\left(T * \theta_{\varepsilon}\right)_{\varepsilon}+\mathcal{N}_{\tau}\left[\mathbb{R}^{n} ; \mathbb{R}\right] \in \mathcal{G}_{\tau}\left(\mathbb{R}^{n} ; \mathbb{R}\right)
$$

e temos que essa aplicação é injetora.

Considere

$$
\begin{gathered}
\mathcal{O}_{C}\left(\mathbb{R}^{n} ; \mathbb{R}\right):=\left\{f \in \mathscr{C}^{\infty}\left(\mathbb{R}^{n} ; \mathbb{R}\right) \mid \exists N \in \mathbb{N}, \forall \alpha \in \mathbb{N}^{n}, \exists c>0\right. \text { tal que } \\
\left.\left|\partial^{\alpha} f(x)\right| \leq c(1+|x|)^{N}, \quad \forall x \in \mathbb{R}^{n}\right\} .
\end{gathered}
$$


É claro que $\mathcal{O}_{C}\left(\mathbb{R}^{n} ; \mathbb{R}\right) \subset \mathcal{E}_{\tau}\left[\mathbb{R}^{n} ; \mathbb{R}\right]$, e assim, pode-se definir

$$
\begin{aligned}
i_{\mathcal{O}_{C}}: \mathcal{O}_{C}\left(\mathbb{R}^{n} ; \mathbb{R}\right) & \longrightarrow \mathcal{G}_{\tau}\left(\mathbb{R}^{n} ; \mathbb{R}\right) \\
f & \longmapsto(f)_{\varepsilon}+\mathcal{N}_{\tau}\left[\mathbb{R}^{n} ; \mathbb{R}\right]
\end{aligned}
$$

Note que $i_{\mathcal{O}_{C}}$ é injetiva, pois, se $(f)_{\varepsilon} \in \mathcal{N}_{\tau}\left[\mathbb{R}^{n} ; \mathbb{R}\right]$, então existem $N \in \mathbb{N}, c>0$ e $\eta \in \mathbf{I}$ tais que

$$
|f(x)| \leq c(1+|x|)^{N} \varepsilon, \quad \forall(\varepsilon, x) \in \mathbf{I}_{\eta} \times \mathbb{R}^{n} .
$$

Logo, fixado $a \in \mathbb{R}^{n}$, tem-se

$$
|f(a)| \leq c(1+|a|)^{N} \varepsilon, \quad \forall \varepsilon \in \mathbf{I}_{\eta}
$$

e portanto $f(a)=0$.

Da Proposição 2.1.21, pode-se considerar a aplicação

$$
\begin{aligned}
\mathcal{O}_{C}\left(\mathbb{R}^{n} ; \mathbb{R}\right) & \longrightarrow \mathcal{S}^{\prime}\left(\mathbb{R}^{n} ; \mathbb{R}\right) \\
f & \longmapsto d_{f}
\end{aligned}
$$

onde $d_{f}$ é a distribuição temperada definida em (2.16). Observe que essa aplicação é injetora, pois se $d_{f}=0$, tem-se que $0=d_{f}(\varphi)=\int_{R^{n}} f(y) \varphi(y) d y$, para toda $\varphi \in \mathcal{S}\left(\mathbb{R}^{n} ; \mathbb{R}\right)$, e portanto para toda $\varphi \in \mathscr{C}_{c}^{\infty}\left(\mathbb{R}^{n} ; \mathbb{R}\right)$. Logo, $f=0$ ([Cor]).

No próximo teorema provaremos que

$$
\left.i_{\mathcal{S}^{\prime}}\right|_{\left\{d_{f} \mid f \in \mathcal{O}_{C}\left(R^{n} ; R\right)\right\}}=i_{\mathcal{O}_{C}} .
$$

Como, o conjunto $\left\{d_{f} \mid f \in \mathcal{O}_{C}\left(\mathbb{R}^{n} ; \mathbb{R}\right)\right\}$ pode ser identificado, como vimos, com o conjunto $\mathcal{O}_{C}\left(\mathbb{R}^{n} ; \mathbb{R}\right)$ escreveremos, para facilitar a escrita, $\left.i_{\mathcal{S}^{\prime}}\right|_{\mathcal{O}_{C}\left(R^{n} ; R\right)}$ ao invés de $\left.i_{\mathcal{S}^{\prime}}\right|_{\left\{d_{f} \mid f \in \mathcal{O}_{C}\left(R^{n} ; R\right)\right\}}$.

Teorema 2.3.3 A aplicação

$$
\begin{aligned}
i_{\mathcal{S}^{\prime}}: \mathcal{S}^{\prime}\left(\mathbb{R}^{n} ; \mathbb{R}\right) & \longrightarrow \mathcal{G}_{\tau}\left(\mathbb{R}^{n} ; \mathbb{R}\right) \\
T & \longmapsto\left(T * \theta_{\varepsilon}\right)_{\varepsilon}+\mathcal{N}_{\tau}\left[\mathbb{R}^{n} ; \mathbb{R}\right]
\end{aligned}
$$

é um homomorfiso injetivo de álgebras. Além disso, tem-se que $\left.i_{\mathcal{S}^{\prime}}\right|_{\mathcal{O}_{C}\left(R^{n} ; R\right)}=i_{\mathcal{O}_{C}}$. 
Demonstração: É claro que $i_{\mathcal{S}^{\prime}}$ é linear. Além disso, da Proposição 2.3.2, tem-se que $i_{\mathcal{S}^{\prime}}$ é injetora. Portanto, para concluir a prova, basta verificar que $\left.i_{\mathcal{S}^{\prime}}\right|_{\mathcal{O}_{C}\left(R^{n} ; R\right)}=i_{\mathcal{O}_{C}}$.

Seja $f \in \mathcal{O}_{C}\left(\mathbb{R}^{n} ; \mathbb{R}\right)$ e considere $\Delta:=i_{\mathcal{S}^{\prime}}\left(d_{f}\right)-i_{\mathcal{O}_{C}}(f)$, onde $d_{f}$ é a distribuição temperada definida em (2.16). Note que um representante de $\Delta$ é dado por

$$
\Delta_{\varepsilon}(x)=\left(d_{f} * \theta_{\varepsilon}\right)(x)-f(x)=\int_{R^{n}} f(y) \theta_{\varepsilon}(x-y) d y-f(x), \quad \forall(\varepsilon, x) \in \mathbf{I} \times \mathbb{R}^{n} .
$$

Da Proposição 2.2.3 (3.), existe $\left(n_{\varepsilon}\right)_{\varepsilon} \in \mathcal{N}(\mathbb{R})$ tal que

$$
\int_{R^{n}} \theta_{\varepsilon}(y) d y=1+n_{\varepsilon}, \quad \forall \varepsilon \in \mathbf{I} .
$$

Portanto, de (2.71), temos que

$$
\Delta_{\varepsilon}(x)=\int_{R^{n}}[f(y)-f(x)] \theta_{\varepsilon}(x-y) d y+f(x) n_{\varepsilon}, \quad \forall(\varepsilon, x) \in \mathbf{I} \times \mathbb{R}^{n} .
$$

Seja $m \in \mathbb{N}$. Como $f \in \mathscr{C}^{\infty}\left(\mathbb{R}^{n} ; \mathbb{R}\right)$ podemos utilizar o polinômio de Taylor de ordem $m+1$ em torno de $x \in \mathbb{R}^{n}$. Para facilitar a escrita desse polinômio denotaremos, para $s \in \mathbb{N} \backslash\{0\}, v=\left(v_{1}, \ldots, v_{n}\right) \in \mathbb{R}^{n}$ e $a=\left(a_{1}, \cdots, a_{n}\right) \in \mathbb{R}^{n}$,

$$
\nabla^{s} f(a) \bullet v^{s}:=\sum_{i_{1}=1}^{n} \sum_{i_{2}=1}^{n} \cdots \sum_{i_{s}=1}^{n} \frac{\partial^{s} f}{\partial x_{i_{1}} \partial x_{i_{2}} \cdots \partial x_{i_{s}}}(a) v_{i_{1}} v_{i_{2}} \cdots v_{i_{s}} .
$$

Portanto, para $y \in \mathbb{R}^{n}$, existe $\sigma_{y} \in[0,1]$ tal que

$$
f(y)-f(x)=\sum_{i=1}^{m+1} \frac{1}{i !} \nabla^{i} f(x) \bullet(y-x)^{i}+\frac{1}{(m+2) !} \nabla^{m+2} f\left(x+\sigma_{y}(x-y)\right) \bullet(y-x)^{m+2} .
$$

Logo, por (2.72), tem-se

$$
\begin{aligned}
\Delta_{\varepsilon}(x)= & \sum_{i=1}^{m+1} \frac{1}{i !} \int_{R^{n}} \nabla^{i} f(x) \bullet(y-x)^{i} \theta_{\varepsilon}(x-y) d y+ \\
& \quad+\int_{R^{n}} \frac{1}{(m+2) !} \nabla^{m+2} f\left(x+\sigma_{y}(x-y)\right) \bullet(y-x)^{m+2} \theta_{\varepsilon}(x-y) d y+f(x) n_{\varepsilon},
\end{aligned}
$$

para $(\varepsilon, x) \in \mathbf{I} \times \mathbb{R}^{n}$.

Como $f \in \mathcal{O}_{C}\left(\mathbb{R}^{n} ; \mathbb{R}\right)$, existe $N \in \mathbb{N}$ tal que

$\forall \alpha \in \mathbb{N}^{n}, \exists c_{\alpha}>0$ tal que $\left|\partial^{\alpha} f(x)\right| \leq c_{\alpha}(1+|x|)^{N}, \quad \forall x \in \mathbb{R}^{n}$. 
Logo, existe $c>0$ satisfazendo

$$
\left|\partial^{\beta} f(x)\right| \leq c(1+|x|)^{N}, \quad \forall x \in \mathbb{R}^{n} \text { e } \forall \beta \in \mathbb{N}^{n} \text { com }|\beta| \leq m+2 .
$$

De $\left(n_{\varepsilon}\right)_{\varepsilon} \in \mathcal{N}(\mathbb{R})$ e $\left(\int_{R^{n}} w^{\beta} \theta_{\varepsilon}(w) d w\right)_{\varepsilon} \in \mathcal{N}(\mathbb{R})$, para todo $\beta \in \mathbb{N}^{n} \backslash\{0\}$ (Proposição 2.2 .3 (4.)), existem $c_{1}>c$ e $\eta_{1} \in \mathbf{I}$ tais que

$$
\begin{gathered}
\left|n_{\varepsilon}\right| \leq c_{1} \varepsilon^{m}, \quad \forall \varepsilon \in \mathbf{I}_{\eta_{1}} ; \\
\left|\int_{R^{n}} w^{\beta} \theta_{\varepsilon}(w) d w\right| \leq c_{1} \varepsilon^{m}, \quad \forall \varepsilon \in \mathbf{I} \text { e } \forall \beta \in \mathbb{N}^{n} \backslash\{0\} \text { com }|\beta| \leq m+2 .
\end{gathered}
$$

Fixe $(\varepsilon, x) \in \mathbf{I}_{\eta_{1}} \times \mathbb{R}^{n}$ e considere

$$
\begin{gathered}
G_{\varepsilon}(x):=\sum_{i=1}^{m+1} \int_{R^{n}} \frac{1}{i !} \nabla^{i} f(x) \bullet(x-y)^{i} \theta_{\varepsilon}(x-y) d y \\
H_{\varepsilon}(x):=\int_{R^{n}} \frac{1}{(m+2) !} \nabla^{m+2} f\left(x+\sigma_{y}(x-y)\right) \bullet(y-x)^{m+2} \theta_{\varepsilon}(x-y) d y .
\end{gathered}
$$

Então,

$$
\left|\Delta_{\varepsilon}(x)\right| \leq\left|G_{\varepsilon}(x)\right|+\left|H_{\varepsilon}(x)\right|+|f(x)|\left|n_{\varepsilon}\right| .
$$

Notemos que

$$
\begin{aligned}
G_{\varepsilon}(x) & =\sum_{i=1}^{m+1} \sum_{|\beta|=i} \frac{1}{i !} \partial^{\beta} f(x) \int_{R^{n}}(x-y)^{\beta} \theta_{\varepsilon}(x-y) d y \\
& =\sum_{i=1}^{m+1} \sum_{|\beta|=i} \frac{1}{i !} \partial^{\beta} f(x) \int_{R^{n}} w^{\beta} \theta_{\varepsilon}(w) d w,
\end{aligned}
$$

e assim, por (2.74)e (2.76), temos que

$$
\left|G_{\varepsilon}(x)\right| \leq\left(\sum_{i=1}^{m+1} \sum_{|\beta|=i} \frac{1}{i !} c_{1}^{2}\right)(1+|x|)^{N} \varepsilon^{m} .
$$

Como

$$
H_{\varepsilon}(x)=\sum_{|\beta|=m+2} \frac{1}{(m+2) !} \int_{R^{n}} \partial^{\beta} f\left(x+\sigma_{y}(x-y)\right)(y-x)^{\beta} \frac{1}{\varepsilon^{n}} \rho\left(\frac{x-y}{\varepsilon}\right) \chi\left(\frac{x-y}{\sqrt{\varepsilon}}\right) d y,
$$


temos, por $(2.74)$ e de $0 \leq \chi \leq 1$, que

$$
\begin{aligned}
\left|H_{\varepsilon}(x)\right| & \leq \sum_{|\beta|=m+2} \frac{1}{(m+2) !} \int_{R^{n}} c\left(1+\left|x+\sigma_{y}(x-y)\right|\right)^{N}\left|(y-x)^{\beta} \frac{1}{\varepsilon^{n}} \rho\left(\frac{x-y}{\varepsilon}\right)\right| d y \\
& \leq \sum_{|\beta|=m+2} \frac{1}{(m+2) !} \int_{R^{n}} c(1+|x|+|x-y|)^{N}\left|(y-x)^{\beta} \frac{1}{\varepsilon^{n}} \rho\left(\frac{x-y}{\varepsilon}\right)\right| d y \\
& \leq \sum_{|\beta|=m+2} \frac{1}{(m+2) !} \int_{R^{n}} c(1+|x|+|\varepsilon w|)^{N} \varepsilon^{|\beta|}\left|w^{\beta} \rho(w)\right| d w \\
& \leq \sum_{|\beta|=m+2} \frac{1}{(m+2) !} \int_{R^{n}} c(1+|x|+|w|)^{N} \varepsilon^{m+2}|w|^{|\beta|}|\rho(w)| d w \\
& \leq \sum_{|\beta|=m+2} \frac{1}{(m+2) !} \int_{R^{n}} c(1+|x|)^{N}(1+|w|)^{N} \varepsilon^{m+2}|w|^{|\beta|}|\rho(w)| d w \\
& \leq\left(\sum_{|\beta|=m+2} \frac{1}{(m+2) !} \int_{R^{n}}(1+|w|)^{N}|w|^{|\beta|}|\rho(w)| d w\right) c(1+|x|)^{N} \varepsilon^{m+2}
\end{aligned}
$$

e assim

$$
\left|H_{\varepsilon}(x)\right| \leq\left(\sum_{|\beta|=m+2} \frac{1}{(m+2) !} \int_{R^{n}}(1+|w|)^{N}|w|^{m+2}|\rho(w)| d w\right) c(1+|x|)^{N} \varepsilon^{m+2}
$$

(note que, pela Observação 2.1.14 (3.), a integral que aparece em (2.79) é finita).

De $(2.75),(2.77),(2.78)$ e $(2.79)$, temos que, se

$$
c_{2}:=\left(\sum_{i=1}^{m+1} \sum_{|\beta|=i} \frac{1}{i !} c_{1}^{2}\right)+\left(\sum_{|\beta|=m+2} \frac{c}{(m+2) !} \int_{R^{n}}(1+|w|)^{N}|w|^{m+2}|\rho(w)| d w\right)+c_{1},
$$

então

$$
\left|\Delta_{\varepsilon}(x)\right| \leq c_{2}(1+|x|)^{N} \varepsilon^{m}, \quad \forall(\varepsilon, x) \in \mathbf{I}_{\eta_{1}} \times \mathbb{R}^{n} .
$$

Logo, pelo Teorema de Nulidade em $\mathcal{E}_{\tau}\left[\mathbb{R}^{n} ; \mathbb{R}\right]$ (Teorema 1.3.7), segue que $\left(\Delta_{\varepsilon}\right) \varepsilon \in \mathcal{N}_{\tau}\left[\mathbb{R}^{n} ; \mathbb{R}\right]$.

É importante observar que na prova do Teorema 2.3.3 foi fundamental o fato de $f$ pertencer a $\mathcal{O}_{C}\left(\mathbb{R}^{n} ; \mathbb{R}\right)$ para exibir um $N$ que não dependesse de $m$. Note que não teríamos como garantir esse fato caso tivéssemos $f \in \mathcal{O}_{M}\left(\mathbb{R}^{n} ; \mathbb{R}\right)$. 
Notemos que, do Exemplo 2.1.19, a Medida de Dirac pode ser vista como um elemento de $\mathcal{S}^{\prime}\left(\mathbb{R}^{n} ; \mathbb{R}\right)$ e, dessa forma, $i_{\mathcal{S}^{\prime}}(\delta) \in \mathcal{G}_{\tau}\left(\mathbb{R}^{n} ; \mathbb{R}\right)$. O Exemplo 2.3.4, a seguir, nos mostrará qual é a classe de $i_{\mathcal{S}^{\prime}}(\delta)$ em $\mathcal{G}_{\tau}\left(\mathbb{R}^{n} ; \mathbb{R}\right)$. Essa classe será útil na seção 2 do Capítulo 3.

Exemplo 2.3.4 Sejam $a \in \mathbb{R}^{n}$ e $\widetilde{\delta}_{a}$ (também denotada por $\delta_{a}$ ) a distribuição temperada definida no Exemplo 2.1.19. Então

$$
i_{\mathcal{S}^{\prime}}\left(\widetilde{\delta}_{a}\right)=\left(x \in \mathbb{R}^{n} \longmapsto \rho_{\varepsilon}(x-a)\right)_{\varepsilon}+\mathcal{N}_{\tau}\left[\mathbb{R}^{n} ; \mathbb{R}\right] .
$$

De fato, basta observar que

$$
\left(\widetilde{\delta}_{a} * \theta_{\varepsilon}\right)(x)=\widetilde{\delta}_{a}\left(\theta_{\varepsilon}(x-\bullet)\right)=\theta_{\varepsilon}(x-a), \quad \forall(\varepsilon, x) \in \mathbf{I} \times \mathbb{R}^{n},
$$

e que, da Proposição 2.2.3 (2.), tem-se que

$$
\left(x \in \mathbb{R}^{n} \longmapsto \theta_{\varepsilon}(x-a)-\rho_{\varepsilon}(x-a)\right)_{\varepsilon} \in \mathcal{N}_{\tau}\left[\mathbb{R}^{n} ; \mathbb{R}\right] .
$$

Finalizamos este capítulo com o seguinte resultado.

Proposição 2.3.5 Seja $\alpha \in \mathbb{N}^{n}$. Então

$$
i_{\mathcal{S}^{\prime}}\left(\partial^{\alpha} T\right)=\partial^{\alpha}\left(i_{\mathcal{S}^{\prime}}(T)\right), \quad \forall T \in \mathcal{S}^{\prime}\left(\mathbb{R}^{n} ; \mathbb{R}\right) .
$$

Demonstração: Notemos que

$$
\begin{gathered}
i_{\mathcal{S}^{\prime}}\left(\partial^{\alpha} T\right)=\left(x \in \mathbb{R}^{n} \longmapsto\left(\partial^{\alpha} T * \theta_{\varepsilon}\right)(x)\right)_{\varepsilon}+\mathcal{N}_{\tau}\left[\mathbb{R}^{n} ; \mathbb{R}\right] ; \\
i_{\mathcal{S}^{\prime}}(T)=\left(x \in \mathbb{R}^{n} \longmapsto\left(T * \theta_{\varepsilon}\right)(x)\right)_{\varepsilon}+\mathcal{N}_{\tau}\left[\mathbb{R}^{n} ; \mathbb{R}\right] .
\end{gathered}
$$

De (2.21) temos, para todo $(\varepsilon, x) \in \mathbf{I} \times \mathbb{R}^{n}$, que

$$
\begin{aligned}
\left(\partial^{\alpha} T * \theta_{\varepsilon}\right)(x) & =\partial^{\alpha} T\left(\theta_{\varepsilon}(x-\bullet)\right)=(-1)^{|\alpha|} T\left(\partial^{\alpha}\left(\theta_{\varepsilon}(x-\bullet)\right)\right) \\
& =T\left(\partial^{\alpha} \theta_{\varepsilon}(x-\bullet)\right)=\left(T * \partial^{\alpha} \theta_{\varepsilon}\right)(x)=\partial^{\alpha}\left(T * \theta_{\varepsilon}\right)(x) .
\end{aligned}
$$

$\operatorname{Logo}, i_{\mathcal{S}^{\prime}}\left(\partial^{\alpha} T\right)=\partial^{\alpha} i_{\mathcal{S}^{\prime}}(T)$. 


\section{Capítulo 3}

\section{Equações diferenciais ordinárias}

Um estudo sobre as equações diferenciais ordinárias no contexto das funções generalizadas temperadas de Colombeau pode ser encontrado na seção 1.5 de [GKOS]. Apresentar esses resultados, de forma detalhada, é o objetivo deste capítulo.

Na seção 3.1, iremos estabelecer algumas condições sobre $F \in \mathcal{G}_{\tau}\left(J \times \mathbb{R}^{n} ; \mathbb{R}^{n}\right)$ ou

$F \in \widetilde{\mathcal{G}}_{\tau}\left(J \times \mathbb{R}^{n} ; \mathbb{R}^{n}\right)$, para que, dados $t_{0} \in \mathbb{R}$ e $x_{0} \in \overline{\mathbb{R}}^{n}$, exista uma (ou apenas uma) aplicação generalizada $u \in \mathcal{G}\left(J ; \mathbb{R}^{n}\right)$ ou, no caso em que $F \in \mathcal{G}_{\tau}\left(J \times \mathbb{R}^{n} ; \mathbb{R}^{n}\right)$, uma (ou apenas uma) aplicação generalizada temperada $u \in \mathcal{G}_{\tau}\left(J ; \mathbb{R}^{n}\right)$ tal que

$$
\frac{d u}{d t}=F \circ(i d, u) \quad \text { e } u\left(t_{0}\right)=x_{0}
$$

onde $J$ é um intervalo aberto de $\mathbb{R}$ e $i d$ denota, no caso de $u \in \mathcal{G}\left(J ; \mathbb{R}^{n}\right)$, a função generalizada definida por

$$
i d:=(t \in J \longmapsto t)_{\varepsilon}+\mathcal{N}[J ; \mathbb{R}]
$$

ou, no caso de $u \in \mathcal{G}_{\tau}\left(J ; \mathbb{R}^{n}\right)$, a função generalizada temperada definida por

$$
i d:=(t \in J \longmapsto t)_{\varepsilon}+\mathcal{N}_{\tau}[J ; \mathbb{R}] .
$$

Note que, a composta $F \circ(i d, u)$ pertence a $\mathcal{G}\left(J ; \mathbb{R}^{n}\right)$ se $u \in \mathcal{G}\left(J ; \mathbb{R}^{n}\right)$ (Definição 1.3.15 e Definição 1.4.10), e pertence a $\mathcal{G}_{\tau}\left(J ; \mathbb{R}^{n}\right)$ se $F \in \mathcal{G}_{\tau}\left(J \times \mathbb{R}^{n} ; \mathbb{R}^{n}\right)$ e $u \in \mathcal{G}_{\tau}\left(J ; \mathbb{R}^{n}\right)$ (Definição 1.3.11). 
A idéia utilizada, para encontrar tais condições sobre $F$, é a de fixar um representante $\left(F_{\varepsilon}\right)_{\varepsilon}$ de $F$ e um representante $\left(x_{0_{\varepsilon}}\right)_{\varepsilon}$ de $x_{0}$, e verificar que propriedades $\left(F_{\varepsilon}\right)_{\varepsilon}$ deve ter para que o problema, a nível de representantes, tenha solução moderada (respectivamente moderada temperada) "definida em $J$ ", isto é, determinar condições para que exista $u_{\varepsilon} \in \mathcal{E}_{M}\left[J ; \mathbb{R}^{n}\right]$ (resp. $\left.\mathcal{E}_{\tau}\left[J ; \mathbb{R}^{n}\right]\right)$ de modo que

$$
\frac{d u_{\varepsilon}}{d t}(t)=F_{\varepsilon}\left(t, u_{\varepsilon}(t)\right) \quad \text { e } \quad u_{\varepsilon}\left(t_{0}\right)=x_{0_{\varepsilon}}, \quad \forall(\varepsilon, t) \in I \times J
$$

A partir desse raciocínio, é fundamental o conhecimento da teoria clássica das Equações Diferenciais Ordinárias. Para facilitar a leitura, os resultados da teoria clássica que utilizaremos estarão enunciados no trabalho. Alguns desses resultados são bem elementares, isto é, são apresentados em qualquer estudo inicial de E.D.O., outros nem tanto. Para os que se enquadram nesse último caso, como os que serão usados na seções 3.2 e 3.3, optamos por demonstrá-los.

Estudaremos, na seção 3.2, uma específica equação de Euler-Lagrange e finalizamos o capítulo, e este trabalho, com resultados sobre a existência e unicidade de solução em $\mathcal{G}\left(\left[t_{0},+\infty\left[; \mathbb{R}^{n}\right)\right.\right.$ e em $\mathcal{G}_{\tau}\left(\left[t_{0},+\infty\left[; \mathbb{R}^{n}\right)\right.\right.$ para sistemas autônomos, isto é, da forma

$$
\frac{d u}{d t}=F \circ u \quad \text { e } \quad u\left(t_{0}\right)=x_{0}
$$

onde $F \in \mathcal{G}_{\tau}\left(\mathbb{R}^{n} ; \mathbb{R}\right)$.

Note que, $F \circ u$ pertence a $\mathcal{G}\left(\left[t_{0},+\infty\left[; \mathbb{R}^{n}\right)\right.\right.$ se $u \in \mathcal{G}\left(\left[t_{0},+\infty\left[; \mathbb{R}^{n}\right)\right.\right.$ (Definição 1.5.10), e pertence a $\mathcal{G}_{\tau}\left(\left[t_{0},+\infty\left[; \mathbb{R}^{n}\right)\right.\right.$ se $u \in \mathcal{G}_{\tau}\left(\left[t_{0},+\infty\left[; \mathbb{R}^{n}\right)\right.\right.$ (Definição 1.5.8).

\subsection{Existência e unicidade de soluções}

Para que possamos apresentar alguns resultados sobre a existência e unicidade de soluções das equações diferenciais ordinárias que serão abordadas nesta seção, precisamos 
antes, apresentar uma série de resultados muito importantes para tal estudo, como por exemplo, condições de crescimento para funções moderadas temperadas (Definição 3.1 .1 e Definição 3.1.7).

Definição 3.1.1 Sejam $\Omega$ um aberto de $\mathbb{R}^{n}$ e $\left(f_{\varepsilon}\right)_{\varepsilon} \in \mathcal{E}_{\tau}\left[\Omega ; \mathbb{R}^{m}\right]$. Dizemos que

(i) $\left(f_{\varepsilon}\right)_{\varepsilon}$ é $L^{\infty}$-tipo em $\mathcal{E}_{\tau}\left[\Omega ; \mathbb{R}^{m}\right]$ se existem $N \in \mathbb{N}, c>0$ e $\eta \in \mathbf{I}$ tais que

$$
\left|f_{\varepsilon}(x)\right| \leq c \varepsilon^{-N}, \quad \forall(\varepsilon, x) \in \mathbf{I}_{\eta} \times \Omega
$$

(ii) $\left(f_{\varepsilon}\right)_{\varepsilon}$ é $L^{\infty}-\ln$-tipo em $\mathcal{E}_{\tau}\left[\Omega ; \mathbb{R}^{m}\right]$ se existem $c>0$ e $\eta \in \mathbf{I}$ tais que

$$
\left|f_{\varepsilon}(x)\right| \leq c|\ln \varepsilon|, \quad \forall(\varepsilon, x) \in \mathbf{I}_{\eta} \times \Omega
$$

Definição 3.1.2 Seja $\Omega$ um aberto de $\mathbb{R}^{n}$. Definimos

$$
\begin{aligned}
& \mathcal{E}_{\tau, L^{\infty}}\left[\Omega ; \mathbb{R}^{m}\right]:=\left\{\left(f_{\varepsilon}\right)_{\varepsilon} \in \mathcal{E}_{\tau}\left[\Omega ; \mathbb{R}^{m}\right] \mid\left(f_{\varepsilon}\right)_{\varepsilon} \text { é } L^{\infty} \text {-tipo em } \mathcal{E}_{\tau}\left[\Omega ; \mathbb{R}^{m}\right]\right\} \\
& \mathcal{E}_{\tau, \ln }\left[\Omega ; \mathbb{R}^{m}\right]:=\left\{\left(f_{\varepsilon}\right)_{\varepsilon} \in \mathcal{E}_{\tau}\left[\Omega ; \mathbb{R}^{m}\right] \mid\left(f_{\varepsilon}\right)_{\varepsilon} \text { é } L^{\infty} \text {-ln -tipo em } \mathcal{E}_{\tau}\left[\Omega ; \mathbb{R}^{m}\right]\right\} .
\end{aligned}
$$

Note que $\mathcal{E}_{\tau, L^{\infty}}\left[\Omega ; \mathbb{R}^{m}\right]=\left(\mathcal{E}_{\tau, L^{\infty}}[\Omega ; \mathbb{R}]\right)^{m}$ e $\mathcal{E}_{\tau, \ln }\left[\Omega ; \mathbb{R}^{m}\right]=\left(\mathcal{E}_{\tau, \ln }[\Omega ; \mathbb{R}]\right)^{m}$. Assim para fornecer exemplos de funções que satisfazem, ou não, a Definição 3.1.1 basta construir exemplos no caso em que $m=1$.

Exemplo 3.1.3 Seja $\Omega$ um aberto de $\mathbb{R}^{n}, r \in \mathbb{N}, \varphi \in \mathscr{C}^{\infty}\left(\mathbb{R}^{n} ; \mathbb{R}\right)$ limitada e não nula $e$ $\left(f_{\varepsilon}\right)_{\varepsilon},\left(g_{\varepsilon}\right)_{\varepsilon} \in \mathcal{E}_{\tau}[\Omega ; \mathbb{R}]$ definidas por

$$
\begin{array}{ll}
f_{\varepsilon}(x)=\ln \varepsilon \varphi\left(\frac{x}{\varepsilon}\right), & \forall x \in \Omega ; \\
g_{\varepsilon}(x)=\frac{1}{\varepsilon^{r}} \varphi\left(\frac{x}{\varepsilon}\right), & \forall x \in \Omega .
\end{array}
$$

Tem-se 
1. $\left(f_{\varepsilon}\right)_{\varepsilon} \in \mathcal{E}_{\tau, \ln }[\Omega ; \mathbb{R}]$

2. $\left(f_{\varepsilon}\right)_{\varepsilon},\left(g_{\varepsilon}\right)_{\varepsilon} \in \mathcal{E}_{\tau, L^{\infty}}[\Omega ; \mathbb{R}]$;

3. se $0 \in \Omega$ e $\varphi(0) \neq 0$, então $\left(g_{\varepsilon}\right)_{\varepsilon} \notin \mathcal{E}_{\tau, \ln }[\Omega ; \mathbb{R}]$;

4. $\left(\theta_{\varepsilon}\right)_{\varepsilon} \in \mathcal{E}_{\tau, L^{\infty}}\left[\mathbb{R}^{n} ; \mathbb{R}\right]$ e $\left(\theta_{\varepsilon}\right)_{\varepsilon} \notin \mathcal{E}_{\tau, \ln }\left[\mathbb{R}^{n} ; \mathbb{R}\right]$, onde $\left(\theta_{\varepsilon}\right)_{\varepsilon}$ é como em $(2.25)$.

De fato, basta usar a Definição 3.1.1 e observar, para as três primeiras, que $\varphi$ é limitada, que $\lim _{\varepsilon \downarrow 0}(\varepsilon|\ln \varepsilon|)=0$ e que $\lim _{\varepsilon \downarrow 0} \frac{|\varphi(0)|}{\varepsilon^{r}|\ln \varepsilon|}=+\infty$, se $0 \in \Omega$ e $\varphi(0) \neq 0$. Para obter 4., além da Definição 3.1.1, usar que

$$
\left|\theta_{\varepsilon}(x)\right|=\frac{1}{\varepsilon^{n}}\left|\rho\left(\frac{x}{\varepsilon}\right)\right|\left|\chi\left(\frac{x}{\sqrt{\varepsilon}}\right)\right| \leq \frac{1}{\varepsilon^{n}}\left|\rho\left(\frac{x}{\varepsilon}\right)\right| ;
$$

que, se $a \in \mathbb{R}^{n}$ é tal que $\rho(a) \neq 0$, então

$$
\left|\theta_{\varepsilon}(\varepsilon a)\right|=\left|\frac{1}{\varepsilon^{n}} \rho(a)\right|, \quad \text { para } 0<\varepsilon<\left(\frac{1}{1+|a|}\right)^{2} ;
$$

que $\rho$ é limitada e que $\lim _{\varepsilon \downarrow 0} \frac{|\rho(a)|}{\varepsilon^{n}|\ln \varepsilon|}=+\infty$.

Os conjuntos $\mathcal{E}_{\tau, L^{\infty}}\left[\Omega ; \mathbb{R}^{m}\right]$ e $\mathcal{E}_{\tau, \ln }\left[\Omega ; \mathbb{R}^{m}\right]$ com as operações usuais de soma de funções e produto de número real por função são $\mathbb{R}$-espaços vetoriais. Além disso, tem-se,

Proposição 3.1.4 Seja $\Omega$ um aberto de $\mathbb{R}^{n}$. Então

1. $\mathcal{E}_{\tau, L^{\infty}}[\Omega ; \mathbb{R}]$ é uma $\mathbb{R}$-álgebra;

2. $\mathcal{E}_{\tau, \ln }\left[\Omega ; \mathbb{R}^{m}\right] \varsubsetneqq \mathcal{E}_{\tau, L^{\infty}}\left[\Omega ; \mathbb{R}^{m}\right]$;

3. se $\left(f_{\varepsilon}\right)_{\varepsilon} \in \mathcal{E}_{\tau, \ln }[\Omega ; \mathbb{R}]$ e $\left(h_{\varepsilon}\right)_{\varepsilon} \in \mathcal{E}_{\tau, L^{\infty}}[\Omega ; \mathbb{R}]$, então $\left(f_{\varepsilon} h_{\varepsilon}\right)_{\varepsilon} \in \mathcal{E}_{\tau, L^{\infty}}[\Omega ; \mathbb{R}]$.

Demonstração: A asserção 1. é imediata. A afirmação 3. e que $\mathcal{E}_{\tau, \ln }\left[\Omega ; \mathbb{R}^{m}\right] \subset \mathcal{E}_{\tau, L^{\infty}}\left[\Omega ; \mathbb{R}^{m}\right]$ segue de $\lim _{\varepsilon \downarrow 0}(\varepsilon|\ln \varepsilon|)=0$. Para concluir a prova basta observar que, se $\left(g_{\varepsilon}\right)_{\varepsilon} \in \mathcal{E}_{\tau}[\Omega ; \mathbb{R}]$ é definida por $g_{\varepsilon}(x)=\frac{1}{\varepsilon}$, então $\left(\left(g_{\varepsilon}, 0 \cdots, 0\right)\right)_{\varepsilon} \in \mathcal{E}_{\tau, L^{\infty}}\left[\Omega ; \mathbb{R}^{m}\right] \backslash \mathcal{E}_{\tau, \ln }\left[\Omega ; \mathbb{R}^{m}\right]$. 
Definição 3.1.5 Seja $\Omega$ um aberto de $\mathbb{R}^{n}$ e $f \in \mathcal{G}_{\tau}\left(\Omega ; \mathbb{R}^{m}\right)$. Dizemos que

(i) fé $L^{\infty}$-tipo em $\mathcal{G}_{\tau}\left(\Omega ; \mathbb{R}^{m}\right)$ se, e somente se, f tem um representante que é $L^{\infty}$-tipo em $\mathcal{E}_{\tau}\left[\Omega ; \mathbb{R}^{m}\right]$

(ii) $f$ é $L^{\infty}-\ln$-tipo em $\mathcal{G}_{\tau}\left(\Omega ; \mathbb{R}^{m}\right)$ se, e somente se, f tem um representante que é $L^{\infty}-\ln$-tipo em $\mathcal{E}_{\tau}\left[\Omega ; \mathbb{R}^{m}\right]$.

Exemplo 3.1.6 Sejam $f \in \mathscr{C}^{\infty}(\mathbb{R} ; \mathbb{R})$ uma função limitada, com $f(0) \neq 0$, e $r \in \mathbb{N}$. Então a função

$$
g:=\left(x \longmapsto \frac{1}{\varepsilon^{r}} f\left(\frac{x}{\varepsilon}\right)\right)_{\varepsilon}+\mathcal{N}_{\tau}[\mathbb{R} ; \mathbb{R}]
$$

é $L^{\infty}$-tipo em $\mathcal{G}_{\tau}(\mathbb{R} ; \mathbb{R})$ mas não é $L^{\infty}$ - $\ln$-tipo em $\mathcal{G}_{\tau}(\mathbb{R} ; \mathbb{R})$.

De fato, seja $\left(g_{\varepsilon}\right)_{\varepsilon}$ definida por

$$
g_{\varepsilon}(x):=\frac{1}{\varepsilon^{r}} f\left(\frac{x}{\varepsilon}\right), \quad \forall(\varepsilon, x) \in \mathbf{I} \times \mathbb{R} .
$$

É claro que $\left(g_{\varepsilon}\right)_{\varepsilon} \in \mathcal{E}_{\tau, L^{\infty}}[\mathbb{R} ; \mathbb{R}]$, e assim $g$ é $L^{\infty}$-tipo em $\mathcal{G}_{\tau}(\mathbb{R} ; \mathbb{R})$. Suponha, por absurdo, que exista $\left(h_{\varepsilon}\right)_{\varepsilon}$ representante de $g$ tal que $\left(h_{\varepsilon}\right)_{\varepsilon}$ seja $L^{\infty}$ - ln-tipo em $\mathcal{E}_{\tau}[\mathbb{R} ; \mathbb{R}]$. Então existe $N \in \mathbb{N}, c>0$ e $\eta \in \mathbf{I}$ tais que

$$
\begin{gathered}
\left|h_{\varepsilon}(x)-g_{\varepsilon}(x)\right| \leq c(1+|x|)^{N} \varepsilon, \quad \forall(\varepsilon, x) \in \mathbf{I}_{\eta} \times \mathbb{R} ; \\
\left|h_{\varepsilon}(x)\right| \leq c|\ln \varepsilon|, \quad \forall(\varepsilon, x) \in \mathbf{I}_{\eta} \times \mathbb{R} .
\end{gathered}
$$

De (3.1) e (3.2) temos que

$$
0<|f(0)| \leq\left|\varepsilon^{r} g_{\varepsilon}(0)\right| \leq \varepsilon^{r}\left(\left|g_{\varepsilon}(0)-h_{\varepsilon}(0)\right|+\left|h_{\varepsilon}(0)\right|\right) \leq \varepsilon^{r}(c \varepsilon+c \ln \varepsilon)
$$

e assim

$$
0<|f(0)| \leq \varepsilon^{r}(c \varepsilon+c \ln \varepsilon), \quad \forall \varepsilon \in \mathbf{I}_{\eta},
$$

o que é um absurdo, visto que $\lim _{\varepsilon \downarrow 0}\left(\varepsilon^{r+1}+\varepsilon^{r} \ln \varepsilon\right)=0$. 
A seguir, apresentamos condições de crescimento para funções moderadas temperadas na segunda variável. Essas condições são semelhantes às da Definição 3.1.1.

Definição 3.1.7 Sejam $\Omega_{1}$ um aberto de $\mathbb{R}^{p}, \Omega_{2}$ um aberto de $\mathbb{R}^{q} e$ $\left(f_{\varepsilon}\right)_{\varepsilon} \in \widetilde{\mathcal{E}}_{\tau}\left[\Omega_{1} \times \Omega_{2} ; \mathbb{R}^{m}\right]$. Dizemos que

(i) $\left(f_{\varepsilon}\right)_{\varepsilon}$ é $L^{\infty}$-tipo em $\widetilde{\mathcal{E}}_{\tau}\left[\Omega_{1} \times \Omega_{2} ; \mathbb{R}^{m}\right]$ se, para todo $K \subset \subset \Omega_{1}$, existem $N \in \mathbb{N}, c>0$ e $\eta \in \mathbf{I}$ tais que

$$
\left|f_{\varepsilon}\left(x_{1}, x_{2}\right)\right| \leq c \varepsilon^{-N}, \quad \forall\left(\varepsilon, x_{1}, x_{2}\right) \in \mathbf{I}_{\eta} \times K \times \Omega_{2}
$$

(ii) $\left(f_{\varepsilon}\right)_{\varepsilon}$ é $L^{\infty}$-ln-tipo em $\widetilde{\mathcal{E}}_{\tau}\left[\Omega_{1} \times \Omega_{2} ; \mathbb{R}^{m}\right]$ se, para todo $K \subset \subset \Omega_{1}$, existem c $>0$ e $\eta \in \mathbf{I}$ tais que

$$
\left|f_{\varepsilon}\left(x_{1}, x_{2}\right)\right| \leq c|\ln \varepsilon|, \quad \forall\left(\varepsilon, x_{1}, x_{2}\right) \in \mathbf{I}_{\eta} \times K \times \Omega_{2}
$$

Definição 3.1.8 Sejam $\Omega_{1}$ um aberto de $\mathbb{R}^{p}$ e $\Omega_{2}$ um aberto de $\mathbb{R}^{q}$. Definimos

$$
\begin{aligned}
& \widetilde{\mathcal{E}}_{\tau, L^{\infty}}\left[\Omega_{1} \times \Omega_{2} ; \mathbb{R}^{m}\right]:=\left\{\left(f_{\varepsilon}\right)_{\varepsilon} \in \widetilde{\mathcal{E}}_{\tau}\left[\Omega_{1} \times \Omega_{2} ; \mathbb{R}^{m}\right] \mid\left(f_{\varepsilon}\right)_{\varepsilon} \text { é } L^{\infty} \text {-tipo em } \widetilde{\mathcal{E}}_{\tau}\left[\Omega_{1} \times \Omega_{2} ; \mathbb{R}^{m}\right]\right\} ; \\
& \widetilde{\mathcal{E}}_{\tau, \ln }\left[\Omega_{1} \times \Omega_{2} ; \mathbb{R}^{m}\right]:=\left\{\left(f_{\varepsilon}\right)_{\varepsilon} \in \widetilde{\mathcal{E}}_{\tau}\left[\Omega_{1} \times \Omega_{2} ; \mathbb{R}^{m}\right] \mid\left(f_{\varepsilon}\right)_{\varepsilon} \text { é } L^{\infty} \text {-ln -tipo em } \widetilde{\mathcal{E}}_{\tau}\left[\Omega_{1} \times \Omega_{2} ; \mathbb{R}^{m}\right]\right\} .
\end{aligned}
$$

No caso em que $\Omega_{1}=\mathbb{R}^{p}$, tem-se a seguinte proposição.

Proposição 3.1.9 Sejam $\Omega_{2}$ um aberto de $\mathbb{R}^{q}$ e $\left(f_{\varepsilon}\right)_{\varepsilon} \in \widetilde{\mathcal{E}}_{\tau}\left(\mathbb{R}^{p} \times \Omega_{2} ; \mathbb{R}^{m}\right)$.

1. São equivalentes:

(a) $\left(f_{\varepsilon}\right)_{\varepsilon}$ é $L^{\infty}$-tipo em $\widetilde{\mathcal{E}}_{\tau}\left(\mathbb{R}^{p} \times \Omega_{2} ; \mathbb{R}^{m}\right)$;

(b) para todo $T \in \mathbb{N}$, existem $N \in \mathbb{N}, c>0$ e $\eta \in \mathbf{I}$ tais que

$$
\left|f_{\varepsilon}\left(x_{1}, x_{2}\right)\right| \leq c \varepsilon^{-N}, \quad \forall\left(\varepsilon, x_{1}, x_{2}\right) \in \mathbf{I}_{\eta} \times[-T, T]^{p} \times \Omega_{2} .
$$


2. São equivalentes:

(a) $\left(f_{\varepsilon}\right)_{\varepsilon}$ é $L^{\infty}$-ln-tipo em $\widetilde{\mathcal{E}}_{\tau}\left(\mathbb{R}^{p} \times \Omega_{2} ; \mathbb{R}^{m}\right)$;

(b) para todo $T \in \mathbb{N}$, existem $c>0$ e $\eta \in \mathbf{I}$ tais que

$$
\left|f_{\varepsilon}\left(x_{1}, x_{2}\right)\right| \leq c|\ln \varepsilon|, \quad \forall\left(\varepsilon, x_{1}, x_{2}\right) \in \mathbf{I}_{\eta} \times[-T, T]^{p} \times \Omega_{2} .
$$

Demonstração: Basta usar a Definição 3.1.7, observar que, se $K \subset \subset \mathbb{R}^{p}$, então existe $T \in \mathbb{N}$ tal que $K \subset[-T, T]^{p}$, e que todo conjunto da forma $[-r, r]^{p}$, para $r \geq 0$, é um subconjunto compacto de $\mathbb{R}^{p}$.

A seguir, veremos alguns exemplos de funções pertencentes a $\widetilde{\mathcal{E}}_{\tau, L^{\infty}}\left[\Omega_{1} \times \Omega_{2} ; \mathbb{R}\right]$ e a $\quad \widetilde{\mathcal{E}}_{\tau, \mathrm{ln}}\left[\Omega_{1} \times \Omega_{2} ; \mathbb{R}\right]$. A partir desses exemplos é possível construir funções em $\widetilde{\mathcal{E}}_{\tau, L^{\infty}}\left[\Omega_{1} \times \Omega_{2} ; \mathbb{R}^{m}\right]$ e em $\quad \widetilde{\mathcal{E}}_{\tau, \ln }\left[\Omega_{1} \times \Omega_{2} ; \mathbb{R}^{m}\right]$, visto que, esses conjuntos são $\left(\widetilde{\mathcal{E}}_{\tau, L \infty}\left[\Omega_{1} \times \Omega_{2} ; \mathbb{R}\right]\right)^{m}$ e $\left(\widetilde{\mathcal{E}}_{\tau, \ln }\left[\Omega_{1} \times \Omega_{2} ; \mathbb{R}\right]\right)^{m}$, respectivamente.

Exemplo 3.1.10 Sejam $\Omega_{1}$ um aberto de $\mathbb{R}^{p}$ e $\Omega_{2}$ um aberto de $\mathbb{R}^{q}$.

1. Se $\varphi \in \mathscr{C}^{\infty}\left(\mathbb{R}^{p} \times \mathbb{R}^{q} ; \mathbb{R}\right)$ é limitada, $r \in \mathbb{N}$ e $\left(f_{\varepsilon}\right)_{\varepsilon},\left(g_{\varepsilon}\right)_{\varepsilon} \in \widetilde{\mathcal{E}}_{\tau}\left[\Omega_{1} \times \Omega_{2} ; \mathbb{R}\right]$ são dadas por

$$
\begin{gathered}
f_{\varepsilon}(x, y)=\ln \varepsilon \varphi\left(\frac{x}{\varepsilon}, \frac{y}{\varepsilon}\right), \quad \forall(x, y) \in \Omega_{1} \times \Omega_{2} ; \\
g_{\varepsilon}(x, y)=\frac{1}{\varepsilon^{r}} \varphi\left(\frac{x}{\varepsilon}, \frac{y}{\varepsilon}\right), \quad \forall(x, y) \in \Omega_{1} \times \Omega_{2},
\end{gathered}
$$

então

(i) $\left(f_{\varepsilon}\right)_{\varepsilon} \in \widetilde{\mathcal{E}}_{\tau, \ln }\left[\Omega_{1} \times \Omega_{2} ; \mathbb{R}\right]$;

(ii) $\left(f_{\varepsilon}\right)_{\varepsilon},\left(g_{\varepsilon}\right)_{\varepsilon} \in \widetilde{\mathcal{E}}_{\tau, L^{\infty}}\left[\Omega_{1} \times \Omega_{2} ; \mathbb{R}\right]$;

(iii) se $(0,0) \in \Omega_{1} \times \Omega_{2}$ e $\varphi(0,0) \neq 0$, ent $\tilde{a} o\left(g_{\varepsilon}\right)_{\varepsilon} \notin \widetilde{\mathcal{E}}_{\tau, \ln }\left[\Omega_{1} \times \Omega_{2} ; \mathbb{R}\right]$. 
2. $S e \quad\left(u_{\varepsilon}\right)_{\varepsilon} \in \mathcal{E}_{M}\left[\Omega_{1} ; \mathbb{R}\right], \quad\left(v_{\varepsilon}\right)_{\varepsilon} \in \mathcal{E}_{\tau, L^{\infty}}\left[\Omega_{2} ; \mathbb{R}\right], \quad\left(w_{\varepsilon}\right)_{\varepsilon} \in \mathcal{E}_{\tau, \ln }\left[\Omega_{2} ; \mathbb{R}\right] \quad e$ $\left(h_{\varepsilon}\right)_{\varepsilon},\left(\Phi_{\varepsilon}\right)_{\varepsilon} \in \widetilde{\mathcal{E}}_{\tau}\left[\Omega_{1} \times \Omega_{2} ; \mathbb{R}\right]$ são dadas por

$$
\begin{gathered}
h_{\varepsilon}(x, y)=u_{\varepsilon}(x) v_{\varepsilon}(y), \quad \forall(x, y) \in \Omega_{1} \times \Omega_{2} ; \\
\Phi_{\varepsilon}(x, y)=w_{\varepsilon}(y), \quad \forall(x, y) \in \Omega_{1} \times \Omega_{2},
\end{gathered}
$$

então

$(i v)\left(h_{\varepsilon}\right)_{\varepsilon} \in \widetilde{\mathcal{E}}_{\tau, L^{\infty}}\left[\Omega_{1} \times \Omega_{2} ; \mathbb{R}\right]$

$(v)\left(\Phi_{\varepsilon}\right)_{\varepsilon} \in \widetilde{\mathcal{E}}_{\tau, \ln }\left[\Omega_{1} \times \Omega_{2} ; \mathbb{R}\right]$

De fato, basta usar a Definição 3.1.7, a Definição 3.1.1 e observar, para 1., que $\lim _{\varepsilon \downarrow 0}(\varepsilon|\ln \varepsilon|)=0$ e que $\lim _{\varepsilon \downarrow 0} \frac{|\varphi(0,0)|}{\varepsilon^{r}|\ln \varepsilon|}=+\infty$ se $(0,0) \in \Omega_{1} \times \Omega_{2}$ e $\varphi(0,0) \neq 0$.

Os conjuntos $\widetilde{\mathcal{E}}_{\tau, L^{\infty}}\left[\Omega_{1} \times \Omega_{2} ; \mathbb{R}\right]$ e $\widetilde{\mathcal{E}}_{\tau, \ln }\left[\Omega_{1} \times \Omega_{2} ; \mathbb{R}\right]$ com as operações usuais de soma de funções e produto de número real por função são $\mathbb{R}$-espaços vetoriais. Além disso, tem-se,

Proposição 3.1.11 Sejam $\Omega_{1}$ um aberto de $\mathbb{R}^{p}$ e $\Omega_{2}$ um aberto de $\mathbb{R}^{q}$. Então

1. $\widetilde{\mathcal{E}}_{\tau, L^{\infty}}\left[\Omega_{1} \times \Omega_{2} ; \mathbb{R}\right]$ é uma $\mathbb{R}$-álgebra;

2. $\widetilde{\mathcal{E}}_{\tau, \ln }\left[\Omega_{1} \times \Omega_{2} ; \mathbb{R}^{m}\right] \varsubsetneqq \widetilde{\mathcal{E}}_{\tau, L^{\infty}}\left[\Omega_{1} \times \Omega_{2} ; \mathbb{R}^{m}\right]$;

3. $s e\left(f_{\varepsilon}\right)_{\varepsilon} \in \widetilde{\mathcal{E}}_{\tau, \ln }\left[\Omega_{1} \times \Omega_{2} ; \mathbb{R}\right]$ e $\left(g_{\varepsilon}\right)_{\varepsilon} \in \widetilde{\mathcal{E}}_{\tau, L^{\infty}}\left[\Omega_{1} \times \Omega_{2} ; \mathbb{R}\right]$, então

$$
\left(f_{\varepsilon} g_{\varepsilon}\right)_{\varepsilon} \in \widetilde{\mathcal{E}}_{\tau, L^{\infty}}\left[\Omega_{1} \times \Omega_{2} ; \mathbb{R}\right]
$$

Demonstração: Análoga à da Proposição 3.1.4 substituindo $\Omega$ por $\Omega_{1} \times \Omega_{2}$.

Definição 3.1.12 Sejam $\Omega_{1}$ um aberto de $\mathbb{R}^{p}, \quad \Omega_{2}$ um aberto de $\mathbb{R}^{q} e$ $f \in \widetilde{\mathcal{G}}_{\tau}\left(\Omega_{1} \times \Omega_{2} ; \mathbb{R}^{m}\right)$. Dizemos que 
(i) $f$ é $L^{\infty}$-tipo em $\widetilde{\mathcal{G}}_{\tau}\left(\Omega_{1} \times \Omega_{2} ; \mathbb{R}^{m}\right)$ se, e somente se, $f$ tem um representante que é $L^{\infty}$-tipo em $\widetilde{\mathcal{E}}_{\tau}\left[\Omega_{1} \times \Omega_{2} ; \mathbb{R}^{m}\right]$;

(ii) f é $L^{\infty}$-ln-tipo em $\widetilde{\mathcal{G}}_{\tau}\left(\Omega_{1} \times \Omega_{2} ; \mathbb{R}^{m}\right)$ se, e somente se, $f$ tem um representante que é $L^{\infty}$-ln -tipo em $\widetilde{\mathcal{E}}_{\tau}\left[\Omega_{1} \times \Omega_{2} ; \mathbb{R}^{m}\right]$.

Exemplo 3.1.13 $\operatorname{Seja}\left(g_{\varepsilon}\right)_{\varepsilon} \in\left(\mathscr{C}^{\infty}(\mathbb{R} \times \mathbb{R} ; \mathbb{R})\right)^{\mathbf{I}}$ definida por

$$
g_{\varepsilon}(t, x):=\frac{1}{\varepsilon}, \quad \forall(\varepsilon, t, x) \in \mathbf{I} \times \mathbb{R} \times \mathbb{R} .
$$

\section{Então}

1. $g:=\left[\left(g_{\varepsilon}\right)_{\varepsilon}\right] \in \mathcal{G}_{\tau}(\mathbb{R} \times \mathbb{R} ; \mathbb{R})$ não é $L^{\infty}$-ln-tipo em $\mathcal{G}_{\tau}(\mathbb{R} \times \mathbb{R} ; \mathbb{R})$;

2. $g:=\left[\left(g_{\varepsilon}\right)_{\varepsilon}\right] \in \widetilde{\mathcal{G}}_{\tau}(\mathbb{R} \times \mathbb{R} ; \mathbb{R})$ não é $L^{\infty}$-ln -tipo em $\widetilde{\mathcal{G}}_{\tau}(\mathbb{R} \times \mathbb{R} ; \mathbb{R})$.

De fato, é claro que $\left(g_{\varepsilon}\right)_{\varepsilon} \in \mathcal{E}_{\tau}[\mathbb{R} \times \mathbb{R} ; \mathbb{R}]$, e portanto $\left(g_{\varepsilon}\right)_{\varepsilon} \in \widetilde{\mathcal{E}}_{\tau}[\mathbb{R} \times \mathbb{R} ; \mathbb{R}]$ (Proposição 1.4.11 (1.)). Para 1., observe que, se $\left(n_{\varepsilon}\right)_{\varepsilon} \in \mathcal{N}_{\tau}[\mathbb{R} \times \mathbb{R} ; \mathbb{R}]$ e $\left(n_{\varepsilon}+g_{\varepsilon}\right)_{\varepsilon} \in \mathcal{E}_{\tau, \ln }[\mathbb{R} \times \mathbb{R} ; \mathbb{R}]$, então existem $p>0, c>0$ e $\eta \in \mathbf{I}$ tais que

$$
\begin{gathered}
\left|n_{\varepsilon}(0, x)\right| \leq c \varepsilon(1+|x|)^{p}, \quad \forall(\varepsilon, x) \in \mathbf{I}_{\eta} \times \mathbb{R} ; \\
\frac{1}{\varepsilon}\left|1+\varepsilon n_{\varepsilon}(0, x)\right|=\left|g_{\varepsilon}(0, x)+n_{\varepsilon}(0, x)\right| \leq c|\ln \varepsilon|, \quad \forall(\varepsilon, x) \in \mathbf{I}_{\eta} \times \mathbb{R} ;
\end{gathered}
$$

e assim

$$
1 \leq\left|1+\varepsilon n_{\varepsilon}(0,0)\right|+\varepsilon\left|n_{\varepsilon}(0,0)\right| \leq c \varepsilon|\ln \varepsilon|+c \varepsilon^{2}, \quad \forall \varepsilon \in \mathbf{I}_{\eta} ;
$$

o que é um absurdo, pois $\lim _{\varepsilon \downarrow 0}\left(\varepsilon|\ln \varepsilon|+\varepsilon^{2}\right)=0$. Logo $\left(n_{\varepsilon}+g_{\varepsilon}\right)_{\varepsilon} \notin \mathcal{E}_{\tau, \ln }[\mathbb{R} \times \mathbb{R} ; \mathbb{R}]$, para todo $\left(n_{\varepsilon}\right)_{\varepsilon} \in \mathcal{N}_{\tau}[\mathbb{R} \times \mathbb{R} ; \mathbb{R}]$, ou seja, $g$ não é $L^{\infty}$-ln-tipo em $\mathcal{G}_{\tau}(\mathbb{R} \times \mathbb{R} ; \mathbb{R})$. Note que 2. é obtido de modo análogo a 1. considerando $K=\{0\}, \quad\left(n_{\varepsilon}\right)_{\varepsilon} \in \widetilde{\mathcal{N}}_{\tau}[\mathbb{R} \times \mathbb{R} ; \mathbb{R}]$ e $\left(n_{\varepsilon}+g_{\varepsilon}\right)_{\varepsilon} \in \widetilde{\mathcal{E}}_{\tau, \ln }[\mathbb{R} \times \mathbb{R} ; \mathbb{R}]$. 
Exemplo 3.1.14 Seja $\left(g_{\varepsilon}\right)_{\varepsilon} \in\left(\mathscr{C}^{\infty}(\mathbb{R} \times \mathbb{R} ; \mathbb{R})\right)^{\mathbf{I}}$ definida por

$$
g_{\varepsilon}(t, x):=\frac{1}{\varepsilon}\left(\operatorname{sech}\left(\frac{x}{\varepsilon}\right)\right)^{2}, \quad \forall(\varepsilon, t, x) \in \mathbf{I} \times \mathbb{R} \times \mathbb{R} .
$$

\section{Então}

1. $g:=\left[\left(g_{\varepsilon}\right)_{\varepsilon}\right] \in \mathcal{G}_{\tau}(\mathbb{R} \times \mathbb{R} ; \mathbb{R})$ não é $L^{\infty}$ - $\ln$-tipo em $\mathcal{G}_{\tau}(\mathbb{R} \times \mathbb{R} ; \mathbb{R})$;

2. $g:=\left[\left(g_{\varepsilon}\right)_{\varepsilon}\right] \in \widetilde{\mathcal{G}}_{\tau}(\mathbb{R} \times \mathbb{R} ; \mathbb{R})$ não é $L^{\infty}$ - $\ln$-tipo em $\widetilde{\mathcal{G}}_{\tau}(\mathbb{R} \times \mathbb{R} ; \mathbb{R})$.

De fato, é claro que $\left(g_{\varepsilon}\right)_{\varepsilon} \in \mathcal{E}_{\tau}[\mathbb{R} \times \mathbb{R} ; \mathbb{R}]$, e portanto $\left(g_{\varepsilon}\right)_{\varepsilon} \in \widetilde{\mathcal{E}}_{\tau}[\mathbb{R} \times \mathbb{R} ; \mathbb{R}]$ (Proposição 1.4 .11 (1.) ). Para 1., note que se, $\left(n_{\varepsilon}\right)_{\varepsilon} \in \mathcal{N}_{\tau}[\mathbb{R} \times \mathbb{R} ; \mathbb{R}]$ e $\left(n_{\varepsilon}+g_{\varepsilon}\right)_{\varepsilon} \in \mathcal{E}_{\tau, \ln }[\mathbb{R} \times \mathbb{R} ; \mathbb{R}]$, então existem $p>0, c>0$ e $\eta \in \mathbf{I}$ tais que

$$
\begin{gathered}
\left|n_{\varepsilon}(0, x)\right| \leq c \varepsilon(1+|x|)^{p}, \quad \forall(\varepsilon, x) \in \mathbf{I}_{\eta} \times \mathbb{R} ; \\
\frac{1}{\varepsilon}\left|\left(\operatorname{sech}\left(\frac{x}{\varepsilon}\right)\right)^{2}+\varepsilon n_{\varepsilon}(0, x)\right|=\left|g_{\varepsilon}(0, x)+n_{\varepsilon}(0, x)\right| \leq c|\ln \varepsilon|, \quad \forall(\varepsilon, x) \in \mathbf{I}_{\eta} \times \mathbb{R} .
\end{gathered}
$$

Assim

$$
1=\left|\operatorname{sech}\left(\frac{0}{\varepsilon}\right)\right|^{2} \leq\left|\left(\operatorname{sech}\left(\frac{0}{\varepsilon}\right)\right)^{2}+\varepsilon n_{\varepsilon}(0,0)\right|+\left|\varepsilon n_{\varepsilon}(0,0)\right| \leq c \varepsilon|\ln \varepsilon|+c \varepsilon^{2}, \quad \forall \varepsilon \in \mathbf{I}_{\eta},
$$

o que é um absurdo, pois $\lim _{\varepsilon \downarrow 0}\left(\varepsilon|\ln \varepsilon|+\varepsilon^{2}\right)=0$. Logo, $\left(n_{\varepsilon}+g_{\varepsilon}\right)_{\varepsilon} \notin \mathcal{E}_{\tau, \ln }[\mathbb{R} \times \mathbb{R} ; \mathbb{R}]$, para todo $\left(n_{\varepsilon}\right)_{\varepsilon} \in \mathcal{N}_{\tau}[\mathbb{R} \times \mathbb{R} ; \mathbb{R}]$, ou seja, $g$ não é $L^{\infty}$-ln-tipo em $\mathcal{G}_{\tau}(\mathbb{R} \times \mathbb{R} ; \mathbb{R})$. Observe que 2. é obtido de modo análogo a 1. considerando $K=\{0\}, \quad\left(n_{\varepsilon}\right)_{\varepsilon} \in \widetilde{\mathcal{N}}_{\tau}[\mathbb{R} \times \mathbb{R} ; \mathbb{R}]$ e $\left(n_{\varepsilon}+g_{\varepsilon}\right)_{\varepsilon} \in \widetilde{\mathcal{E}}_{\tau, \ln }[\mathbb{R} \times \mathbb{R} ; \mathbb{R}]$.

Na Proposição 3.1 .15 veremos uma relação direta entre a Definição 3.1 .1 e a Definição 3.1.7, substituindo o aberto $\Omega$ da Definição 3.1 .1 por um aberto da forma $\Omega_{1} \times \Omega_{2}$.

Proposição 3.1.15 Sejam $\Omega_{1}$ um aberto de $\mathbb{R}^{p}$ e $\Omega_{2}$ um aberto de $\mathbb{R}^{q}$. Tem-se que

1. $\mathcal{E}_{\tau, L^{\infty}}\left[\Omega_{1} \times \Omega_{2} ; \mathbb{R}^{m}\right] \subset \widetilde{\mathcal{E}}_{\tau, L^{\infty}}\left[\Omega_{1} \times \Omega_{2} ; \mathbb{R}^{m}\right]$

2. $\mathcal{E}_{\tau, \ln }\left[\Omega_{1} \times \Omega_{2} ; \mathbb{R}^{m}\right] \subset \widetilde{\mathcal{E}}_{\tau, \ln }\left[\Omega_{1} \times \Omega_{2} ; \mathbb{R}^{m}\right]$. 
Demonstração: Decorre da definição de cada conjunto.

Após este breve estudo sobre condições de crescimento para funções moderadas temperadas e moderadas temperadas na segunda variável, iremos nos dedicar ao propósito desta seção, isto é, estudaremos, sob quais condições é garantida, a existência de uma (ou somente uma) solução generalizada ou solução generalizada temperada, para o problema

$$
\begin{aligned}
\frac{d u}{d t} & =F \circ(i d, u) \\
u\left(t_{0}\right) & =x_{0}
\end{aligned}
$$

onde $F \in \widetilde{\mathcal{G}}_{\tau}\left(J \times \mathbb{R}^{n} ; \mathbb{R}^{n}\right)$ ou $F \in \mathcal{G}_{\tau}\left(J \times \mathbb{R}^{n} ; \mathbb{R}^{n}\right), J$ é um intervalo aberto de $\mathbb{R}, t_{0} \in J$ e $x_{0} \in \overline{\mathbb{R}}^{n}$ e $i d$ denota, no caso de (3.3) ter uma solução generalizada, a função generalizada definida por

$$
i d:=(t \in J \longmapsto t)_{\varepsilon}+\mathcal{N}[J ; \mathbb{R}]
$$

ou, no caso de (3.3) ter uma solução generalizada temperada, a função generalizada temperada definida por

$$
i d:=(t \in J \longmapsto t)_{\varepsilon}+\mathcal{N}_{\tau}[J ; \mathbb{R}]
$$

No estudo da existência e unicidade de solução, no contexto clássico, de Equações Diferenciais Ordinárias, utiliza-se com freqüência o seguinte Lema:

Lema de Gronwall: Sejam $u, v:[a, b] \longrightarrow \mathbb{R}$ continuas e $k \in \mathbb{R}$. Se $v \geq 0$ e $u(t) \leq k+\int_{a}^{t} u(s) v(s) d s$ para todo $t \in[a, b]$, então $u(t) \leq k \exp \left(\int_{a}^{t} v(s) d s\right)$, para todo $t \in[a, b]$.

Estudando a demonstração do Lema de Gronwall, encontrada em [FS], verificamos que ele pode ser escrito como faremos no Lema 3.1.16. Essa formulação será útil para as demonstrações dos principais resultados deste trabalho. 
Lema 3.1.16 Sejam $J$ um intervalo aberto limitado de $\mathbb{R}, u, v: J \longrightarrow \mathbb{R}$ contínuas, $k \in \mathbb{R}$ e c $\in J$. Se $u \geq 0, v \geq 0$ e $u(t) \leq k+\left|\int_{c}^{t} u(s) v(s) d s\right|$ para todo $t \in J$, então

$$
u(t) \leq k \exp \left(\left|\int_{c}^{t} v(s) d s\right|\right), \text { para todo } t \in J
$$

Demonstração: Notemos que, se $J$ é um intervalo aberto limitado de $\mathbb{R}$, então existem $a, b \in \mathbb{R}$ tais que $J=] a, b[$. Além disso, se $t \in[c, b[$, então

$$
\left|\int_{c}^{t} u(s) v(s) d s\right|=\int_{c}^{t} u(s) v(s) d s
$$

pois, $u \geq 0$ e $v \geq 0$. Assim

$$
u(t) \leq k+\int_{c}^{t} u(s) v(s) d s, \text { para todo } t \in[c, b[.
$$

Seja $\varphi$ a função definida em $[c, b[$ por

$$
\varphi(t):=k+\int_{c}^{t} u(s) v(s) d s .
$$

Observe que

$$
\frac{d \varphi}{d t}(t)=u(t) v(t) \leq \varphi(t) v(t), \text { para todo } t \in[c, b[,
$$

e assim,

$$
\frac{d}{d t}\left(\exp \left(-\int_{c}^{t} v(s) d s\right) \varphi(t)\right) \leq 0, \text { para todo } t \in[c, b[
$$

Logo, a função

$$
t \in\left[c, b\left[\longmapsto \exp \left(-\int_{c}^{t} v(s) d s\right) \varphi(t)\right.\right.
$$

é não-crescente para todo $t \in[c, b[$, ou seja

$$
\exp \left(-\int_{c}^{t} v(s) d s\right) \varphi(t) \leq \varphi(c)=k, \text { para todo } t \in[c, b[.
$$

Portanto

$$
u(t) \leq \varphi(t) \leq k \exp \left(\int_{c}^{t} v(s) d s\right), \text { para todo } t \in[c, b[
$$

Note que, se $t \in] a, c]$, então

$$
\left|\int_{c}^{t} u(s) v(s) d s\right|=\left|-\int_{t}^{c} u(s) v(s) d s\right|=\int_{t}^{c} u(s) v(s) d s
$$


pois, $u \geq 0$ e $v \geq 0$. Assim

$$
\left.\left.u(t) \leq k+\int_{t}^{c} u(s) v(s) d s, \text { para todo } t \in\right] a, c\right] .
$$

Seja $w$ a função definida em ] $a, c]$ por

$$
w(t):=-\left(k+\int_{t}^{c} u(s) v(s) d s\right) .
$$

Observe que

$$
\left.\left.\frac{d w}{d t}(t)=u(t) v(t) \leq-w(t) v(t), \text { para todo } t \in\right] a, c\right]
$$

e assim,

$$
\left.\left.\frac{d}{d t}\left(\exp \left(-\int_{t}^{c} v(s) d s\right) w(t)\right) \leq 0, \text { para todo } t \in\right] a, c\right]
$$

Logo, a função

$$
t \in] a, c] \longmapsto \exp \left(-\int_{t}^{c} v(s) d s\right) w(t)
$$

é não-crescente para todo $t \in] a, c]$. Logo

$$
\left.\left.\exp \left(-\int_{t}^{c} v(s) d s\right) w(t) \geq w(c)=-k, \text { para todo } t \in\right] a, c\right]
$$

ou seja,

$$
\left.\left.u(t) \leq-w(t) \leq k \exp \left(\int_{t}^{c} v(s) d s\right)=k \exp \left(-\int_{c}^{t} v(s) d s\right), \text { para todo } t \in\right] a, c\right]
$$

De (3.4) e (3.5) tem-se

$$
u(t) \leq k \exp \left(\left|\int_{c}^{t} v(s) d s\right|\right), \text { para todo } t \in J .
$$

Iniciamos nosso estudo apresentando algumas propriedades de $\left(u_{\varepsilon}\right)_{\varepsilon} \in\left(\mathscr{C}^{\infty}\left(J ; \mathbb{R}^{n}\right)\right)^{\mathbf{I}}$ tal que

$$
\frac{d u_{\varepsilon}}{d t}(x)=F_{\varepsilon}\left(t, u_{\varepsilon}(t)\right), \quad \forall(\varepsilon, t) \in \mathbf{I} \times J,
$$

onde $J$ é um intervalo aberto de $\mathbb{R}$ e $\left(F_{\varepsilon}\right)_{\varepsilon} \in \widetilde{\mathcal{E}}_{\tau}\left[J \times \mathbb{R}^{n} ; \mathbb{R}^{n}\right]$ ou $\left(F_{\varepsilon}\right)_{\varepsilon} \in \mathcal{E}_{\tau}\left[J \times \mathbb{R}^{n} ; \mathbb{R}^{n}\right]$. 
Proposição 3.1.17 Sejam $J$ um intervalo aberto de $\mathbb{R}, \quad\left(u_{\varepsilon}\right)_{\varepsilon} \in\left(\mathscr{C}^{\infty}\left(J ; \mathbb{R}^{n}\right)\right)^{\mathbf{I}}$ $e\left(F_{\varepsilon}\right)_{\varepsilon} \in \widetilde{\mathcal{E}}_{\tau}\left[J \times \mathbb{R}^{n} ; \mathbb{R}^{n}\right]$ tais que

(i) $\frac{d u_{\varepsilon}}{d t}(t)=F_{\varepsilon}\left(t, u_{\varepsilon}(t)\right), \quad \forall(\varepsilon, t) \in \mathbf{I} \times J ;$

(ii) $\forall K \subset \subset J, \exists N_{1} \in \mathbb{N}, \exists c_{1}>0 e \exists \eta_{1} \in \mathbf{I}$ satisfazendo

$$
\left|u_{\varepsilon}(t)\right| \leq c_{1} \varepsilon^{-N_{1}}, \quad \forall(\varepsilon, t) \in \mathbf{I}_{\eta_{1}} \times K .
$$

$\operatorname{Ent} \tilde{a} o\left(u_{\varepsilon}\right)_{\varepsilon} \in \mathcal{E}_{M}\left[J ; \mathbb{R}^{n}\right]$

Demonstração: Seja $K \subset \subset J$. Provaremos, pelo Princípio de Indução Finita, que para todo $p \in \mathbb{N}$, existem $N \in \mathbb{N}, c>0$ e $\eta \in \mathbf{I}$ tais que

$$
\left|\frac{d^{p} u_{\varepsilon}}{d t^{p}}(t)\right| \leq c \varepsilon^{-N}, \quad \forall(\varepsilon, t) \in \mathbf{I}_{\eta} \times K,
$$

e como $K \subset \subset \Omega$ é arbitrário, concluíremos que $\left(u_{\varepsilon}\right)_{\varepsilon} \in \mathcal{E}_{M}\left[J ; \mathbb{R}^{n}\right]$.

Seja $p \in \mathbb{N}$. Da hipótese $(i i)$, existem $N_{1} \in \mathbb{N}, c_{1}>0$ e $\eta_{1} \in \mathbf{I}$ satisfazendo (3.6). Como $\left(F_{\varepsilon}\right)_{\varepsilon} \in \widetilde{\mathcal{E}}_{\tau}\left[J \times \mathbb{R}^{n} ; \mathbb{R}^{n}\right]$, existem $N_{2} \in \mathbb{N}$ com $N_{2}>N_{1}, c_{2}>c_{1}$ e $\eta_{2} \in \mathbf{I}$ com $\eta_{2}<\eta_{1}$ tais que

$$
\left|\partial^{\beta} F_{\varepsilon}(t, y)\right| \leq c_{2}(1+|y|)^{N_{2}} \varepsilon^{-N_{2}}, \quad \forall(\varepsilon, t, y) \in \mathbf{I}_{\eta_{2}} \times K \times \mathbb{R}^{n} \text { e } \forall \beta \in \mathbb{N}^{n+1} \operatorname{com}|\beta| \leq p
$$

De (3.6) e (3.8) temos, para quaisquer $(\varepsilon, t) \in \mathbf{I}_{\eta_{2}} \times K$ e $\beta \in \mathbb{N}^{n+1}$ com $|\beta| \leq p$, que

$$
\begin{aligned}
\left|\partial^{\beta} F_{\varepsilon}\left(t, u_{\varepsilon}(t)\right)\right| & \leq c_{2}\left(1+\left|u_{\varepsilon}(t)\right|\right)^{N_{2}} \varepsilon^{-N_{2}} \\
& \leq c_{2}\left(1+c_{1} \varepsilon^{-N_{1}}\right)^{N_{2}} \varepsilon^{-N_{2}} \\
& \leq c_{2}\left(\varepsilon^{-N_{1}}+c_{1} \varepsilon^{-N_{1}}\right)^{N_{2}} \varepsilon^{-N_{2}} \\
& \leq c_{2}\left(1+c_{1}\right) \varepsilon^{-\left(N_{1} N_{2}+N_{2}\right)} \\
& \leq c_{2}\left(1+c_{2}\right)^{N_{2}} \varepsilon^{-\left(N_{2}^{2}+N_{2}\right)} .
\end{aligned}
$$

Portanto, para todo $\beta \in \mathbb{N}^{n+1} \operatorname{com}|\beta| \leq p$, temos

$$
\left|\partial^{\beta} F_{\varepsilon}\left(t, u_{\varepsilon}(t)\right)\right| \leq c_{2}\left(1+c_{2}\right)^{N_{2}} \varepsilon^{-\left(N_{2}^{2}+N_{2}\right)}, \quad \forall(\varepsilon, t) \in \mathbf{I}_{\eta_{2}} \times K
$$


Note que, por (3.6), $(i)$ e (3.9) com $|\beta|=0$, tem-se que (3.7) é verdadeira para $p=0$ ou $p=1$.

Suponhamos $p \geq 2$ e que (3.7) seja verdadeira para todo $s \in \mathbb{N}$ com $s \leq p-1$. Então existem $N_{3} \in \mathbb{N}, c_{3}>0$ e $\eta_{3} \in \mathbf{I}$ com $\eta_{3}<\eta_{2}$ tais que

$$
\left|\frac{d^{s} u_{\varepsilon}}{d t^{s}}(t)\right| \leq c_{3} \varepsilon^{-N_{3}}, \quad \forall(\varepsilon, t) \in \mathbf{I}_{\eta_{3}} \times K \text { e } \forall s \in \mathbb{N} \text { com } s \leq p-1 .
$$

Admita que $\left(u_{\varepsilon}\right)_{\varepsilon}:=\left(\left(u_{1_{\varepsilon}}, \cdots, u_{n_{\varepsilon}}\right)\right)_{\varepsilon}$ e $\left(F_{\varepsilon}\right)_{\varepsilon}:=\left(\left(F_{1_{\varepsilon}}, \cdots, F_{n_{\varepsilon}}\right)\right)_{\varepsilon}$. Para $(\varepsilon, t) \in \mathbf{I}_{\eta_{3}} \times K$ fixado, considere os conjuntos

$$
\begin{gathered}
A:=\left\{\frac{d^{s} u_{i_{\varepsilon}}}{d t^{s}}(t) \mid 0 \leq s \leq p-1 \text { e } 1 \leq i \leq n\right\} ; \\
B:=\left\{\partial^{\beta} F_{i_{\varepsilon}}\left(t, u_{\varepsilon}(t)\right)|| \beta \mid \leq p-1 \text { e } 1 \leq i \leq n\right\} .
\end{gathered}
$$

De $(i)$ observamos que cada coordenada de $\frac{d^{p} u_{\varepsilon}}{d t^{p}}(t)$ é soma e produto de funções que pertencem a $A \cup B$. Portanto, de (3.9) e (3.10), temos que existem $N_{4} \in \mathbb{N}$ e $c_{4}>0$ tais que

$$
\left|\frac{d^{p} u_{\varepsilon}}{d t^{p}}(t)\right| \leq c_{4} \varepsilon^{-N_{4}}, \quad \forall(\varepsilon, t) \in \mathbf{I}_{\eta_{3}} \times K
$$

Logo, (3.7) é verdadeira para $p$.

Para $\left(F_{\varepsilon}\right)_{\varepsilon} \in \mathcal{E}_{\tau}\left[J \times \mathbb{R}^{n} ; \mathbb{R}^{n}\right]$ temos um resultado semelhante ao da Proposição 3.1.17. Mais precisamente,

Proposição 3.1.18 Sejam $J$ um intervalo aberto de $\mathbb{R}, \quad\left(u_{\varepsilon}\right)_{\varepsilon} \in\left(\mathscr{C}^{\infty}\left(J ; \mathbb{R}^{n}\right)\right)^{\mathbf{I}} e$ $\left(F_{\varepsilon}\right)_{\varepsilon} \in \mathcal{E}_{\tau}\left[J \times \mathbb{R}^{n} ; \mathbb{R}^{n}\right]$ tais que

$$
\frac{d u_{\varepsilon}}{d t}(t)=F_{\varepsilon}\left(t, u_{\varepsilon}(t)\right), \quad \forall(\varepsilon, t) \in \mathbf{I} \times J .
$$

São verdadeiras as seguintes afirmações:

1. se existem $N_{1} \in \mathbb{N}, c_{1}>0$ e $\eta_{1} \in \mathbf{I}$ satisfazendo

$$
\left|u_{\varepsilon}(t)\right| \leq c_{1} \varepsilon^{-N_{1}}, \quad \forall(\varepsilon, t) \in \mathbf{I}_{\eta_{1}} \times J
$$


então $\left(u_{\varepsilon}\right)_{\varepsilon} \in \mathcal{E}_{M}\left[J ; \mathbb{R}^{n}\right]$

2. se existem $N_{1} \in \mathbb{N}, c_{1}>0$ e $\eta_{1} \in \mathbf{I}$ satisfazendo

$$
\left|u_{\varepsilon}(t)\right| \leq c_{1}(1+|x|)^{N_{1}} \varepsilon^{-N_{1}}, \quad \forall(\varepsilon, t) \in \mathbf{I}_{\eta_{1}} \times J
$$

então $\left(u_{\varepsilon}\right)_{\varepsilon} \in \mathcal{E}_{\tau}\left[J ; \mathbb{R}^{n}\right]$.

Demonstração: Para 1. basta observar que $\left(F_{\varepsilon}\right)_{\varepsilon} \in \widetilde{\mathcal{E}}_{\tau}\left[J \times \mathbb{R}^{n} ; \mathbb{R}^{n}\right]$ (Proposição 1.4.11 (1.)) e usar a Proposição 3.1.17. Para obter 2. proceda como na prova da Proposição 3.1.17, substitutindo (3.7) por

$$
\left|\frac{d^{p} u_{\varepsilon}}{d t^{p}}(t)\right| \leq c(1+|t|)^{N} \varepsilon^{-N}, \quad \forall(\varepsilon, t) \in \mathbf{I}_{\eta} \times J,
$$

substituindo (ii) pela hipótese de 2., substituindo (3.8) por

$$
\left|\partial^{\beta} F_{\varepsilon}(t, y)\right| \leq c_{2}(1+|y|)^{N_{2}} \varepsilon^{-N_{2}}, \quad \forall(\varepsilon, t, y) \in \mathbf{I}_{\eta_{2}} \times J \times \mathbb{R}^{n} \text { e } \forall \beta \in \mathbb{N}^{n+1} \text { com }|\beta| \leq p,
$$

substituindo (3.9) por

$$
\left|\partial^{\beta} F_{\varepsilon}\left(t, u_{\varepsilon}(t)\right)\right| \leq c_{2}\left(1+\left|c_{1}\right|\right)^{N_{2}}(1+|t|)^{N_{2}^{2}+N_{2}} \varepsilon^{-\left(N_{2}^{2}+N_{2}\right)}, \quad \forall(\varepsilon, t) \in \mathbf{I}_{\eta_{2}} \times J
$$

e substituindo (3.10) por

$$
\left|\frac{d^{s} u_{\varepsilon}}{d t^{s}}(t)\right| \leq c_{3}(1+|t|)^{N_{3}} \varepsilon^{-N_{3}}, \quad \forall(\varepsilon, t) \in \mathbf{I}_{\eta_{2}} \times J \text { e } \forall s \in \mathbb{N} \text { com } s \leq p-1 .
$$

A partir daqui utilizaremos a seguinte notação.

Seja $g:=\left(g_{1}, \cdots, g_{n}\right) \in \mathscr{C}^{\infty}\left(J \times \Omega_{2} ; \mathbb{R}^{m}\right)$, onde $J$ é um intervalo aberto de $\mathbb{R}$ e $\Omega_{2}$ um aberto de $\mathbb{R}^{q}$. Denotamos por $\nabla_{x} g$ o elemento de $\left(\mathscr{C}^{\infty}\left(J \times \Omega_{2} ; \mathbb{R}\right)\right)^{m q}$ definido por

$$
\nabla_{x} g:=\left(\frac{\partial g_{1}}{\partial x_{1}}, \cdots, \frac{\partial g_{1}}{\partial x_{q}}, \frac{\partial g_{2}}{\partial x_{1}}, \cdots, \frac{\partial g_{2}}{\partial x_{q}}, \cdots, \frac{\partial g_{m}}{\partial x_{1}}, \cdots, \frac{\partial g_{m}}{\partial x_{q}}\right)
$$

onde $\quad x:=\left(x_{1}, \cdots, x_{q}\right) \quad$ e $(t, x):=\left(t, x_{1}, \cdots, x_{q}\right)$ denotam um ponto arbitrário de $\mathbb{R}^{q} e$ de $\mathbb{R} \times \mathbb{R}^{q}$, respectivamente. 
Note que, se $\left(h_{\varepsilon}\right)_{\varepsilon}$ e $\left(g_{\varepsilon}\right)_{\varepsilon}$ são representantes de $h \in \mathcal{G}_{\tau}\left(J \times \Omega_{2} ; \mathbb{R}^{m}\right)$ (respectivamente $\left.\widetilde{\mathcal{G}}_{\tau}\left(J \times \Omega_{2} ; \mathbb{R}^{m}\right)\right)$, então $\left(\nabla_{x} h_{\varepsilon}-\nabla_{x} g_{\varepsilon}\right)_{\varepsilon} \in \mathcal{N}_{\tau}\left[J \times \Omega_{2} ; \mathbb{R}^{m q}\right] \quad\left(\operatorname{resp} . \quad \widetilde{\mathcal{N}}_{\tau}\left[J \times \Omega_{2} ; \mathbb{R}^{m q}\right]\right)$. Dessa forma podemos definir o seguinte:

Se $h \in \mathcal{G}_{\tau}\left(J \times \Omega_{2} ; \mathbb{R}^{m}\right)$ (respectivamente $\widetilde{\mathcal{G}}_{\tau}\left(J \times \Omega_{2} ; \mathbb{R}^{m}\right)$ ) denotaremos por $\nabla_{x} h$ a classe de $\left(\nabla_{x} h_{\varepsilon}\right)_{\varepsilon}$ em $\mathcal{G}_{\tau}\left(J \times \Omega_{2} ; \mathbb{R}^{m q}\right)$ (resp. $\left.\widetilde{\mathcal{G}}_{\tau}\left(J \times \Omega_{2} ; \mathbb{R}^{m q}\right)\right)$, onde $\left(h_{\varepsilon}\right)_{\varepsilon}$ é um representante qualquer de $h$.

Nos próximos resultados estabeleceremos condições para que exista $\left(u_{\varepsilon}\right)_{\varepsilon}$ moderada tal que

$$
u_{\varepsilon} \in \mathscr{C}^{\infty}(J ; \mathbb{R}) \quad \text { e } \frac{d u_{\varepsilon}}{d t}(t)=F_{\varepsilon}\left(t, u_{\varepsilon}(t)\right), \quad \forall(\varepsilon, t) \in \mathbf{I} \times J .
$$

Antes de garantirmos a moderação de $\left(u_{\varepsilon}\right)_{\varepsilon}$ precisamos obter condições para que (3.12) seja verdadeira. Recorrendo à teoria clássica das Equações Diferenciais Ordinárias temos, utilizando o Teorema 56-B de [Sim], o seguinte.

Se $U$ é um aberto de $\mathbb{R}$ e $g \in \mathscr{C}^{\infty}\left(U \times \mathbb{R}^{n} ; \mathbb{R}^{n}\right)$ é tal que $\nabla_{x} g$ é limitado em $[c, d] \times \mathbb{R}^{n}$, para todo $[c, d] \subset U$, então, dados $t_{0} \in U$ e $w_{0} \in \mathbb{R}^{n}$, existe uma única solução $w \in \mathscr{C}^{\infty}\left(U ; \mathbb{R}^{n}\right)$ tal que

$$
w\left(t_{0}\right)=w_{0} \quad \text { e } \quad \frac{d w}{d t}(t)=g(t, w(t)), \quad \forall t \in \mathbb{R}^{n} .
$$

Dessa forma, é fácil verificar que

Se $U$ é um aberto de $\mathbb{R}, t_{0} \in U$ e $\left(x_{0_{\varepsilon}}\right)_{\varepsilon} \in\left(\mathbb{R}^{\mathbf{I}}\right)^{n} e\left(F_{\varepsilon}\right)_{\varepsilon} \in \widetilde{\mathcal{E}}_{\tau}\left[U \times \mathbb{R}^{n} ; \mathbb{R}^{n}\right]$ é tal que $\left(\nabla_{x} F_{\varepsilon}\right)_{\varepsilon}$ é $L^{\infty}$-tipo em $\widetilde{\mathcal{E}}_{\tau}\left[U \times \mathbb{R}^{n} ; \mathbb{R}^{n}\right]$, então existe $\left(u_{\varepsilon}\right)_{\varepsilon} \in\left(\mathscr{C}^{\infty}\left(U ; \mathbb{R}^{n}\right)\right)^{\mathbf{I}}$ satisfazendo

$$
u_{\varepsilon}\left(t_{0}\right)=x_{0_{\varepsilon}} \text { e } \frac{d u_{\varepsilon}}{d t}(t)=F_{\varepsilon}\left(t, u_{\varepsilon}(t)\right), \quad \forall(\varepsilon, t) \in \mathbf{I} \times U .
$$

Logo, para termos (3.12), é razoável impor que $\left(\nabla_{x} F_{\varepsilon}\right)_{\varepsilon}$ seja $L^{\infty}$-tipo. Contudo, como veremos mais adiante (Observação 3.1.24), isso não será suficiente para que $\left(u_{\varepsilon}\right)_{\varepsilon}$ seja moderada. Veremos, a seguir, que a moderação será garantida se $\left(\nabla_{x} F_{\varepsilon}\right)_{\varepsilon}$ for $L^{\infty}$ - $\ln$-tipo. 
Proposição 3.1.19 Sejam $J$ um intervalo aberto de $\mathbb{R}, t_{0} \in J,\left(x_{0_{\varepsilon}}\right)_{\varepsilon} \in\left(\mathcal{E}_{M}(\mathbb{R})\right)^{n} e$ $\left(F_{\varepsilon}\right)_{\varepsilon} \in \widetilde{\mathcal{E}}_{\tau}\left[J \times \mathbb{R}^{n} ; \mathbb{R}^{n}\right]$ tal que $\left(\nabla_{x} F_{\varepsilon}\right)_{\varepsilon}$ é $L^{\infty}$-ln -tipo em $\widetilde{\mathcal{E}}_{\tau}\left[J \times \mathbb{R}^{n} ; \mathbb{R}^{n^{2}}\right]$. Então existe $\left(u_{\varepsilon}\right)_{\varepsilon} \in\left(\mathscr{C}^{\infty}\left(J ; \mathbb{R}^{n}\right)\right)^{\mathbf{I}}$ tal que

1. $\frac{d u_{\varepsilon}}{d t}(t)=F_{\varepsilon}\left(t, u_{\varepsilon}(t)\right), \quad \forall(\varepsilon, t) \in \mathbf{I} \times J$

2. $u_{\varepsilon}\left(t_{0}\right)=x_{0_{\varepsilon}}, \quad \forall \varepsilon \in \mathbf{I}$;

3. $\left(u_{\varepsilon}\right)_{\varepsilon} \in \mathcal{E}_{M}\left[J ; \mathbb{R}^{n}\right]$.

Demonstração: As afirmações 1. e 2. seguem de (3.13). Para 3., utilizando a Proposição 3.1.17, basta provarmos que (3.6) é verdadeira. É isso que faremos a seguir.

Seja $K \subset \subset J$. Considere $a, b \in \mathbb{R}$ e $T \in \mathbb{N}^{*}$ tais que

$$
\left.K \cup\left\{t_{0}\right\} \subset\right] a, b[\subset[a, b] \subset \subset J \quad \text { e } \quad[a, b] \subset[-T, T]
$$

De $\quad\left(x_{0_{\varepsilon}}\right)_{\varepsilon} \in\left(\mathcal{E}_{M}(\mathbb{R})\right)^{n}, \quad\left(F_{\varepsilon}\right)_{\varepsilon} \in \widetilde{\mathcal{E}}_{\tau}\left[J \times \mathbb{R}^{n} ; \mathbb{R}^{n}\right]$ e $\left(\nabla_{x} F_{\varepsilon}\right)_{\varepsilon} \quad$ ser $\quad L^{\infty}$-ln-tipo em $\widetilde{\mathcal{E}}_{\tau}\left[J \times \mathbb{R}^{n} ; \mathbb{R}^{n^{2}}\right]$, existem $N_{1} \in \mathbb{N}, c_{1}>0$ e $\eta \in \mathbf{I}$ tais que

$$
\begin{gathered}
\left|x_{0_{\varepsilon}}\right| \leq c_{1} \varepsilon^{-N_{1}}, \quad \forall \varepsilon \in \mathbf{I}_{\eta} ; \\
\left|F_{\varepsilon}(t, y)\right| \leq c_{1}(1+|y|)^{N_{1}} \varepsilon^{-N_{1}}, \quad \forall(\varepsilon, t, y) \in \mathbf{I}_{\eta} \times[a, b] \times \mathbb{R}^{n} ; \\
\left|\nabla_{x} F_{\varepsilon}(t, y)\right| \leq c_{1}|\ln \varepsilon|, \quad \forall(\varepsilon, t, y) \in \mathbf{I}_{\eta} \times[a, b] \times \mathbb{R}^{n} .
\end{gathered}
$$

De 1. e 2. tem-se

$$
u_{\varepsilon}(t)=x_{0_{\varepsilon}}+\int_{t_{0}}^{t} F_{\varepsilon}\left(s, u_{\varepsilon}(s)\right) d s, \quad \forall(\varepsilon, t) \in \mathbf{I} \times J
$$

ou seja,

$$
u_{i_{\varepsilon}}(t)=x_{0_{i_{\varepsilon}}}+\int_{t_{0}}^{t} F_{i_{\varepsilon}}\left(s, u_{\varepsilon}(s)\right) d s, \quad \forall(\varepsilon, t) \in \mathbf{I} \times J \text { e } 1 \leq i \leq n,
$$

onde $\left(u_{\varepsilon}\right)_{\varepsilon}:=\left(\left(u_{1_{\varepsilon}}, \cdots, u_{n_{\varepsilon}}\right)\right)_{\varepsilon},\left(x_{0_{\varepsilon}}\right)_{\varepsilon}:=\left(\left(x_{0_{1_{\varepsilon}}}, \cdots, x_{0_{n_{\varepsilon}}}\right)\right)_{\varepsilon}$ e $\left(F_{\varepsilon}\right)_{\varepsilon}:=\left(\left(F_{1_{\varepsilon}}, \cdots, F_{n_{\varepsilon}}\right)\right)_{\varepsilon}$.

Fixemos $1 \leq i \leq n$. 
Para facilitar a escrita, considere $\left(G_{\varepsilon}\right)_{\varepsilon}:=\left(F_{i_{\varepsilon}}\right)_{\varepsilon}$. Então, de (3.14), (3.15), (3.16) e (3.17), temos que

$$
\begin{gathered}
\left|x_{0_{i_{\varepsilon}}}\right| \leq c_{1} \varepsilon^{-N_{1}}, \quad \forall \varepsilon \in \mathbf{I}_{\eta} ; \\
\left|G_{\varepsilon}(t, y)\right| \leq c_{1}(1+|y|)^{N_{1}} \varepsilon^{-N_{1}}, \quad \forall(\varepsilon, t, y) \in \mathbf{I}_{\eta} \times[a, b] \times \mathbb{R}^{n} ; \\
\left|\nabla_{x} G_{\varepsilon}(t, y)\right| \leq c_{1}|\ln \varepsilon|, \quad \forall(\varepsilon, t, y) \in \mathbf{I}_{\eta} \times[a, b] \times \mathbb{R}^{n} ; \\
u_{i_{\varepsilon}}(t)=x_{0_{i_{\varepsilon}}}+\int_{t_{0}}^{t} G_{\varepsilon}\left(s, u_{\varepsilon}(s)\right) d s, \quad \forall(\varepsilon, t) \in \mathbf{I} \times J .
\end{gathered}
$$

Fixemos $\left.(\varepsilon, t) \in \mathbf{I}_{\eta} \times\right] a, b[$. Como

$$
\int_{t_{0}}^{t} G_{\varepsilon}\left(s, u_{\varepsilon}(s)\right) d s=\int_{t_{0}}^{t}\left(G_{\varepsilon}(s, 0)+G_{\varepsilon}\left(s, u_{\varepsilon}(s)\right)-G_{\varepsilon}(s, 0)\right) d s,
$$

temos, pelo Teorema do Valor Médio, que

$$
\int_{t_{0}}^{t} G_{\varepsilon}\left(s, u_{\varepsilon}(s)\right) d s=\int_{t_{0}}^{t} G_{\varepsilon}(s, 0) d s+\int_{t_{0}}^{t}\left\langle\left(0, u_{\varepsilon}(s)\right),\left(\frac{d G_{\varepsilon}}{d t}, \nabla_{x} G_{\varepsilon}\right)\left(s, \sigma_{s} u_{\varepsilon}(s)\right)\right\rangle d s,
$$

para algum $\sigma_{s} \in[0,1]$, onde $<,>$ denota o produto interno usual de $\mathbb{R}^{n+1}$. Logo

$$
u_{i_{\varepsilon}}(t)=x_{0_{i_{\varepsilon}}}+\int_{t_{0}}^{t} G_{\varepsilon}(s, 0) d s+\int_{t_{0}}^{t}\left\langle u_{\varepsilon}(s), \nabla_{x} G_{\varepsilon}\left(s, \sigma_{s} u_{\varepsilon}(s)\right)\right\rangle d s,
$$

para algum $\sigma_{s} \in[0,1]$.

Por (3.18) e (3.19) temos

$$
\begin{aligned}
\left|x_{0_{i_{\varepsilon}}}+\int_{t_{0}}^{t} G_{\varepsilon}(s, 0) d s\right| & \leq\left|x_{0_{i_{\varepsilon}}}\right|+\left|\int_{t_{0}}^{t}\right| G_{\varepsilon}(s, 0) d s|| \\
& \leq c_{1} \varepsilon^{-N_{1}}+\left|\int_{t_{0}}^{t} c_{1}(1+|0|)^{N_{1}} \varepsilon^{-N_{1}} d s\right| \\
& \leq c_{1} \varepsilon^{-N_{1}}+c_{1} \varepsilon^{-N_{1}}\left|t-t_{0}\right| \\
& \leq c_{1}(1+2 T) \varepsilon^{-N_{1}},
\end{aligned}
$$

e por (3.20) temos

$$
\begin{aligned}
\left|\int_{t_{0}}^{t}\left\langle u_{\varepsilon}(s), \nabla_{x} G_{\varepsilon}\left(s, \sigma_{s} u_{\varepsilon}(s)\right)\right\rangle d s\right| & \leq\left|\int_{t_{0}}^{t}\right| u_{\varepsilon}(s)|| \nabla_{x} G_{\varepsilon}\left(s, \sigma_{s} u_{\varepsilon}(s)\right)|d s| \\
& \leq\left|\int_{t_{0}}^{t}\right| u_{\varepsilon}(s)\left|c_{1}\right| \ln \varepsilon|d s| .
\end{aligned}
$$


Portanto, de (3.22), temos que

$$
\begin{aligned}
\left|u_{i_{\varepsilon}}(t)\right| & \leq\left|x_{0_{i_{\varepsilon}}}+\int_{t_{0}}^{t} G_{\varepsilon}(s, 0) d s\right|+\left|\int_{t_{0}}^{t}\left\langle u_{\varepsilon}(s), \nabla_{x} G_{\varepsilon}\left(s, \sigma_{s} u_{\varepsilon}(s)\right)\right\rangle d s\right| \\
& \leq c_{2} \varepsilon^{-N_{1}}+\left|\int_{t_{0}}^{t}\right| u_{\varepsilon}(s)\left|c_{1}\right| \ln \varepsilon|d s|
\end{aligned}
$$

onde $c_{2}:=c_{1}(1+2 T)$.

Como $\left.(\varepsilon, t) \in \mathbf{I}_{\eta} \times\right] a, b[$ e $1 \leq i \leq n$ são arbitrários, concluímos que

$$
\left.\left|u_{i_{\varepsilon}}(t)\right| \leq c_{2} \varepsilon^{-N_{1}}+\left|\int_{t_{0}}^{t}\right| u_{\varepsilon}(s)\left|c_{1}\right| \ln \varepsilon|d s|, \quad \forall(\varepsilon, t) \in \mathbf{I}_{\eta} \times\right] a, b[
$$

Logo,

$$
\left.\left|u_{\varepsilon}(t)\right| \leq \sum_{i=1}^{n}\left|u_{i_{\varepsilon}}(t)\right| \leq n c_{2} \varepsilon^{-N_{1}}+\left|\int_{t_{0}}^{t}\right| u_{\varepsilon}(s)\left|n c_{1}\right| \ln \varepsilon|d s|, \quad \forall(\varepsilon, t) \in \mathbf{I}_{\eta} \times\right] a, b[
$$

Assim, pelo Lema 3.1.16, obtemos

$$
\begin{aligned}
\left|u_{\varepsilon}(t)\right| & \leq n c_{2} \varepsilon^{-N_{1}} \exp \left(\left|\int_{t_{0}}^{t} n c_{1}\right| \ln \varepsilon|d s|\right) \\
& \leq n c_{2} \varepsilon^{-N_{1}} \exp \left(\left|t-t_{0}\right| n c_{1}|\ln \varepsilon|\right) \\
& \leq n c_{2} \varepsilon^{-N_{1}} \exp \left(2 n T c_{1}|\ln \varepsilon|\right) \\
& \leq n c_{2} \varepsilon^{-N_{1}} \exp \left(\ln \varepsilon^{-2 n T c_{1}}\right) \\
& \leq n c_{2} \varepsilon^{-\left(N_{1}+2 n T c_{1}\right)},
\end{aligned}
$$

para todo $\left.(\varepsilon, t) \in \mathbf{I}_{\eta} \times\right] a, b[$.

Sejam $c:=n c_{2} \quad$ e $\quad N:=N_{1}+2 n T c_{1}$. Então, temos que

$$
\left|u_{\varepsilon}(t)\right| \leq c \varepsilon^{-N}, \quad \forall(\varepsilon, t) \in \mathbf{I}_{\eta} \times K
$$

e, assim (3.6) é verdadeira.

O próximo resultado é para $\left(F_{\varepsilon}\right)_{\varepsilon} \in \mathcal{E}_{\tau}\left[J \times \mathbb{R}^{n} ; \mathbb{R}^{n}\right]$. Veremos que ele é similar ao anterior. 
Proposição 3.1.20 Sejam $J$ um intervalo aberto de $\mathbb{R}, t_{0} \in J,\left(x_{0_{\varepsilon}}\right)_{\varepsilon} \in\left(\mathcal{E}_{M}(\mathbb{R})\right)^{n} e$ $\left(F_{\varepsilon}\right)_{\varepsilon} \in \mathcal{E}_{\tau}\left[J \times \mathbb{R}^{n} ; \mathbb{R}^{n}\right]$ tal que $\left(\nabla_{x} F_{\varepsilon}\right)_{\varepsilon}$ é $L^{\infty}$-ln-tipo em $\mathcal{E}_{\tau}\left[J \times \mathbb{R}^{n} ; \mathbb{R}^{n^{2}}\right]$. Então existe $\left(u_{\varepsilon}\right)_{\varepsilon} \in\left(\mathscr{C}^{\infty}\left(J ; \mathbb{R}^{n}\right)\right)^{\mathbf{I}}$ tal que

1. $\frac{d u_{\varepsilon}}{d t}(t)=F_{\varepsilon}\left(t, u_{\varepsilon}(t)\right), \quad \forall(\varepsilon, t) \in \mathbf{I} \times J ;$

2. $u_{\varepsilon}\left(t_{0}\right)=x_{0_{\varepsilon}}, \quad \forall \varepsilon \in \mathbf{I}$;

3. $\left(u_{\varepsilon}\right)_{\varepsilon} \in \mathcal{E}_{M}\left[J ; \mathbb{R}^{n}\right]$.

Além disso, se $J$ é limitado, então $\left(u_{\varepsilon}\right)_{\varepsilon} \in \mathcal{E}_{\tau}\left[J ; \mathbb{R}^{n}\right]$.

Demonstração: De $\mathcal{E}_{\tau}\left[J \times \mathbb{R}^{n} ; \mathbb{R}^{n}\right] \subset \widetilde{\mathcal{E}}_{\tau}\left[J \times \mathbb{R}^{n} ; \mathbb{R}^{n}\right] \quad($ Proposição 1.4.11 (1.)) e de $\mathcal{E}_{\tau, \ln }\left[J \times \mathbb{R}^{n} ; \mathbb{R}^{n}\right] \subset \widetilde{\mathcal{E}}_{\tau, \ln }\left[J \times \mathbb{R}^{n} ; \mathbb{R}^{n}\right]$ (Proposição 3.1 .15 (2.)) temos, pela Proposição 3.1.19, as afirmações 1., 2. e 3..

Suponhamos $J$ limitado. Verificaremos que existem $N \in \mathbb{N}, c>0$ e $\eta \in \mathbf{I}$ tais que

$$
\left|u_{\varepsilon}(t)\right| \leq c(1+|t|)^{N} \varepsilon^{-N}, \quad \forall(\varepsilon, t) \in \mathbf{I}_{\eta} \times J
$$

e assim, pela Proposição 3.1 .18 (2.), concluiremos que $\left(u_{\varepsilon}\right)_{\varepsilon} \in \mathcal{E}_{\tau}\left[J ; \mathbb{R}^{n}\right]$.

De $\left(x_{0_{\varepsilon}}\right)_{\varepsilon} \in\left(\mathcal{E}_{M}(\mathbb{R})\right)^{n}, \quad\left(F_{\varepsilon}\right)_{\varepsilon} \in \mathcal{E}_{\tau}\left[J \times \mathbb{R}^{n} ; \mathbb{R}^{n}\right] \quad$ e $\left(\nabla_{x} F_{\varepsilon}\right)_{\varepsilon}$ ser $\quad L^{\infty}$-ln-tipo em $\mathcal{E}_{\tau}\left[J \times \mathbb{R}^{n} ; \mathbb{R}^{n^{2}}\right]$, existem $N_{1} \in \mathbb{N}, c_{1}>0$ e $\eta \in \mathbf{I}$ tais que

$$
\begin{gathered}
\left|x_{0_{\varepsilon}}\right| \leq c_{1} \varepsilon^{-N_{1}}, \quad \forall \varepsilon \in \mathbf{I}_{\eta} ; \\
\left|F_{\varepsilon}(t, y)\right| \leq c_{1}(1+|(t, y)|)^{N_{1}} \varepsilon^{-N_{1}}, \quad \forall(\varepsilon, t, y) \in \mathbf{I}_{\eta} \times J \times \mathbb{R}^{n} ; \\
\left|\nabla_{x} F_{\varepsilon}(t, y)\right| \leq c_{1}|\ln \varepsilon|, \quad \forall(\varepsilon, t, y) \in \mathbf{I}_{\eta} \times J \times \mathbb{R}^{n} .
\end{gathered}
$$

De 1. e 2. temos que

$$
u_{\varepsilon}(t)=x_{0_{\varepsilon}}+\int_{t_{0}}^{t} F_{\varepsilon}\left(s, u_{\varepsilon}(s)\right) d s, \quad \forall(\varepsilon, t) \in \mathbf{I} \times J
$$

isto é

$$
u_{i_{\varepsilon}}(t)=x_{0_{i_{\varepsilon}}}+\int_{t_{0}}^{t} F_{i_{\varepsilon}}\left(s, u_{\varepsilon}(s)\right) d s, \quad \forall(\varepsilon, t) \in \mathbf{I} \times J \text { e } 1 \leq i \leq n
$$


onde $\left(u_{\varepsilon}\right)_{\varepsilon}:=\left(\left(u_{1_{\varepsilon}}, \cdots, u_{n_{\varepsilon}}\right)\right)_{\varepsilon},\left(x_{0_{\varepsilon}}\right)_{\varepsilon}:=\left(\left(x_{0_{\varepsilon}}, \cdots, x_{0_{n_{\varepsilon}}}\right)\right)_{\varepsilon}$ e $\left(F_{\varepsilon}\right)_{\varepsilon}:=\left(\left(F_{1_{\varepsilon}}, \cdots, F_{n_{\varepsilon}}\right)\right)_{\varepsilon}$.

Fixemos $1 \leq i \leq n$.

Para facilitar a escrita considere $\left(G_{\varepsilon}\right)_{\varepsilon}:=\left(F_{i_{\varepsilon}}\right)_{\varepsilon}$. Então, de (3.24), (3.25), (3.26) e (3.27), temos que

$$
\begin{gathered}
\left|x_{0_{i_{\varepsilon}}}\right| \leq c_{1} \varepsilon^{-N_{1}}, \quad \forall \varepsilon \in \mathbf{I}_{\eta} ; \\
\left|G_{\varepsilon}(t, y)\right| \leq c_{1}(1+|(t, y)|)^{N_{1}} \varepsilon^{-N_{1}}, \quad \forall(\varepsilon, t, y) \in \mathbf{I}_{\eta} \times J \times \mathbb{R}^{n} ; \\
\left|\nabla_{x} G_{\varepsilon}(t, y)\right| \leq c_{1}|\ln \varepsilon|, \quad \forall(\varepsilon, t, y) \in \mathbf{I}_{\eta} \times J \times \mathbb{R}^{n} ; \\
u_{i_{\varepsilon}}(t)=x_{0_{i_{\varepsilon}}}+\int_{t_{0}}^{t} G_{\varepsilon}\left(s, u_{\varepsilon}(s)\right) d s, \quad \forall(\varepsilon, t) \in \mathbf{I} \times J .
\end{gathered}
$$

Seja $T \in \mathbb{N}^{*}$ tal que $J \subset[-T, T]$.

Fixemos $(\varepsilon, t) \in \mathbf{I}_{\eta} \times J$.

Como

$$
\int_{t_{0}}^{t} G_{\varepsilon}\left(s, u_{\varepsilon}(s)\right) d s=\int_{t_{0}}^{t}\left(G_{\varepsilon}(s, 0)+G_{\varepsilon}\left(s, u_{\varepsilon}(s)\right)-G_{\varepsilon}(s, 0)\right) d s,
$$

temos, pelo Teorema do Valor Médio, que

$$
\int_{t_{0}}^{t} G_{\varepsilon}\left(s, u_{\varepsilon}(s)\right) d s=\int_{t_{0}}^{t} G_{\varepsilon}(s, 0) d s+\int_{t_{0}}^{t}\left\langle\left(0, u_{\varepsilon}(s)\right),\left(\frac{d G_{\varepsilon}}{d t}, \nabla_{x} G_{\varepsilon}\right)\left(s, \sigma_{s} u_{\varepsilon}(s)\right)\right\rangle d s,
$$

para algum $\sigma_{s} \in[0,1]$, onde $\langle$,$\rangle denota o produto interno usual de \mathbb{R}^{n+1}$. Logo

$$
u_{i_{\varepsilon}}(t)=x_{0_{\varepsilon}}+\int_{t_{0}}^{t} G_{\varepsilon}(s, 0) d s+\int_{t_{0}}^{t}\left\langle u_{\varepsilon}(s), \nabla_{x} G_{\varepsilon}\left(s, \sigma_{s} u_{\varepsilon}(s)\right)\right\rangle d s,
$$

para algum $\sigma_{s} \in[0,1]$.

Por (3.28) e (3.29) temos

$$
\begin{aligned}
\left|x_{0_{i_{\varepsilon}}}+\int_{t_{0}}^{t} G_{\varepsilon}(s, 0) d s\right| & \leq\left|x_{0_{i_{\varepsilon}}}\right|+\left|\int_{t_{0}}^{t}\right| G_{\varepsilon}(s, 0) d s|| \\
& \leq c_{1} \varepsilon^{-N_{1}}+\left|\int_{t_{0}}^{t} c_{1}(1+|s|)^{N_{1}} \varepsilon^{-N_{1}} d s\right| \\
& \leq c_{1} \varepsilon^{-N_{1}}+\left|\int_{t_{0}}^{t} c_{1}(1+T)^{N_{1}} \varepsilon^{-N_{1}} d s\right| \\
& \leq c_{1} \varepsilon^{-N_{1}}+c_{1}(1+T)^{N_{1}} \varepsilon^{-N_{1}}\left|t-t_{0}\right| \\
& \leq c_{1} \varepsilon^{-N_{1}}+c_{1}(1+T)^{N_{1}} \varepsilon^{-N_{1}} 2 T \\
& \leq 2 T c_{1}(1+T)^{N_{1}} \varepsilon^{-N_{1}}+2 T c_{1}(1+T)^{N_{1}} \varepsilon^{-N_{1}} \\
& \leq 4 T c_{1}(1+T)^{N_{1}} \varepsilon^{-N_{1}},
\end{aligned}
$$


e por (3.30) temos

$$
\begin{aligned}
\left|\int_{t_{0}}^{t}\left\langle u_{\varepsilon}(s), \nabla_{x} G_{\varepsilon}\left(s, \sigma_{s} u_{\varepsilon}(s)\right)\right\rangle d s\right| & \leq\left|\int_{t_{0}}^{t}\right| u_{\varepsilon}(s)|| \nabla_{x} G_{\varepsilon}\left(s, \sigma_{s} u_{\varepsilon}(s)\right)|d s| \\
& \leq\left|\int_{t_{0}}^{t}\right| u_{\varepsilon}(s)\left|c_{1}\right| \ln \varepsilon|d s| .
\end{aligned}
$$

Logo, de (3.32), temos que

$$
\begin{aligned}
\left|u_{i_{\varepsilon}}(t)\right| & \leq\left|x_{0_{i_{\varepsilon}}}+\int_{t_{0}}^{t} G_{\varepsilon}(s, 0) d s\right|+\left|\int_{t_{0}}^{t}\left\langle u_{\varepsilon}(s), \nabla_{x} G_{\varepsilon}\left(s, \sigma_{s} u_{\varepsilon}(s)\right)\right\rangle d s\right| \\
& \leq c_{2} \varepsilon^{-N_{1}}+\left|\int_{t_{0}}^{t}\right| u_{\varepsilon}(s)\left|c_{1}\right| \ln \varepsilon|d s|
\end{aligned}
$$

onde $c_{2}:=4 T c_{1}(1+T)^{N_{1}}$.

Como $(\varepsilon, t) \in \mathbf{I}_{\eta} \times J$ e $1 \leq i \leq n$ são arbitrários, concluímos que

$$
\left|u_{i \varepsilon}(t)\right| \leq c_{2} \varepsilon^{-N_{1}}+\left|\int_{t_{0}}^{t}\right| u_{\varepsilon}(s)\left|c_{1}\right| \ln \varepsilon|d s|, \quad \forall(\varepsilon, t) \in \mathbf{I}_{\eta} \times J, \quad \forall 1 \leq i \leq n .
$$

Portanto

$$
\left|u_{\varepsilon}(t)\right| \leq \sum_{i=1}^{n}\left|u_{i_{\varepsilon}}(t)\right| \leq n c_{2} \varepsilon^{-N_{1}}+\left|\int_{t_{0}}^{t}\right| u_{\varepsilon}(s)\left|n c_{1}\right| \ln \varepsilon|d s|, \quad \forall(\varepsilon, t) \in \mathbf{I}_{\eta} \times J
$$

Assim, pelo Lema 3.1.16, obtemos

$$
\begin{aligned}
\left|u_{\varepsilon}(t)\right| & \leq n c_{2} \varepsilon^{-N_{1}} \exp \left(\left|\int_{t_{0}}^{t} n c_{1}\right| \ln \varepsilon|d s|\right) \\
& \leq n c_{2} \varepsilon^{-N_{1}} \exp \left(\left|t-t_{0}\right| n c_{1}|\ln \varepsilon|\right) \\
& \leq n c_{2} \varepsilon^{-N_{1}} \exp \left(2 T n c_{1}|\ln \varepsilon|\right) \\
& \leq n c_{2} \varepsilon^{-N_{1}} \exp \left(\ln \varepsilon^{-2 T n c_{1}}\right) \\
& \leq n c_{2} \varepsilon^{-\left(N_{1}+2 T n c_{1}\right)},
\end{aligned}
$$

para todo $(\varepsilon, t) \in \mathbf{I}_{\eta} \times J$.

Sejam $c:=n c_{2} \quad$ e $\quad N:=N_{1}+2 T n c_{1}$. Então

$$
\left|u_{\varepsilon}(t)\right| \leq c \varepsilon^{-N} \leq c(1+|t|)^{N} \varepsilon^{-N}, \quad \forall(\varepsilon, t) \in \mathbf{I}_{\eta} \times J .
$$

Portanto $\left(u_{\varepsilon}\right)_{\varepsilon} \in \mathcal{E}_{\tau}\left[J ; \mathbb{R}^{n}\right]$ (Proposição 3.1 .18 (2.)). 
Veremos, a seguir, que a condição de $\nabla_{x} F$ ser $L^{\infty}$-ln-tipo também garantirá a unicidade de solução em $\mathcal{G}\left(J ; \mathbb{R}^{n}\right)$ para $(3.3)$.

Teorema 3.1.21 Seja J um intervalo aberto de $\mathbb{R}$. São verdadeiras as seguintes afirmações:

1. se $F \in \widetilde{\mathcal{G}}_{\tau}\left(J \times \mathbb{R}^{n} ; \mathbb{R}^{n}\right)$ e existe um representante $\left(F_{\varepsilon}\right)_{\varepsilon}$ de $F$ tal que $\left(\nabla_{x} F_{\varepsilon}\right)_{\varepsilon}$ é $L^{\infty}$-ln-tipo em $\widetilde{\mathcal{E}}_{\tau}\left[J \times \mathbb{R}^{n} ; \mathbb{R}^{n^{2}}\right]$, então o problema (3.3) admite uma única solução em $\mathcal{G}\left(J ; \mathbb{R}^{n}\right)$;

2. se $F \in \mathcal{G}_{\tau}\left(J \times \mathbb{R}^{n} ; \mathbb{R}^{n}\right)$ e existe um representante $\left(F_{\varepsilon}\right)_{\varepsilon}$ de $F$ tal que $\left(\nabla_{x} F_{\varepsilon}\right)_{\varepsilon}$ é $L^{\infty}$-ln -tipo em $\mathcal{E}_{\tau}\left[J \times \mathbb{R}^{n} ; \mathbb{R}^{n^{2}}\right]$, então o problema (3.3) admite uma única solução em $\mathcal{G}\left(J ; \mathbb{R}^{n}\right)$.

Demonstração: Suponhamos $F \in \widetilde{\mathcal{G}}_{\tau}\left(J \times \mathbb{R}^{n} ; \mathbb{R}^{n}\right)$ e seja $\left(F_{\varepsilon}\right)_{\varepsilon}$ um representante de $F$ tal que $\left(\nabla_{x} F_{\varepsilon}\right)_{\varepsilon}$ é $L^{\infty}$-ln-tipo em $\widetilde{\mathcal{E}}_{\tau}\left[J \times \mathbb{R}^{n} ; \mathbb{R}^{n}\right]$. Da Proposição 3.1 .19 , se $\left(x_{0_{\varepsilon}}\right)_{\varepsilon} \in\left(\mathcal{E}_{M}(\mathbb{R})\right)^{n}$ é um representante de $x_{0}$, então existe $\left(u_{\varepsilon}\right)_{\varepsilon} \in\left(\mathscr{C}^{\infty}\left(J ; \mathbb{R}^{n}\right)\right)^{\mathbf{I}}$ tal que $\left(u_{\varepsilon}\right)_{\varepsilon} \in \mathcal{E}_{M}\left[J ; \mathbb{R}^{n}\right]$ e

$$
\begin{aligned}
\frac{d u_{\varepsilon}}{d t}(t) & =F_{\varepsilon}\left(t, u_{\varepsilon}(t)\right), \quad \forall(\varepsilon, t) \in \mathbf{I} \times J \\
u_{\varepsilon}\left(t_{0}\right) & =x_{0_{\varepsilon}} .
\end{aligned}
$$

Assim, $u:=\left(u_{\varepsilon}\right)_{\varepsilon}+\mathcal{N}\left[J ; \mathbb{R}^{n}\right]$ é uma solução de $(3.3)$ em $\mathcal{G}\left(J ; \mathbb{R}^{n}\right)$.

Seja $v:=\left[\left(v_{\varepsilon}\right)_{\varepsilon}\right] \in \mathcal{G}\left(J ; \mathbb{R}^{n}\right)$ uma outra solução para (3.3). Logo existem $\left(n_{\varepsilon}\right)_{\varepsilon} \in \mathcal{N}\left[J ; \mathbb{R}^{n}\right]$ e $\left(m_{\varepsilon}\right)_{\varepsilon} \in(\mathcal{N}(\mathbb{R}))^{n}$ tais que

$$
\begin{aligned}
\frac{d u_{\varepsilon}}{d t}(t)-\frac{d v_{\varepsilon}}{d t}(t) & =F_{\varepsilon}\left(t, u_{\varepsilon}(t)\right)-F_{\varepsilon}\left(t, v_{\varepsilon}(t)\right)+n_{\varepsilon}(t), \quad \forall(\varepsilon, t) \in \mathbf{I} \times J \\
u_{\varepsilon}\left(t_{0}\right)-v_{\varepsilon}\left(t_{0}\right) & =m_{\varepsilon}, \quad \forall \varepsilon \in \mathbf{I} .
\end{aligned}
$$

Provaremos que $\left(u_{\varepsilon}-v_{\varepsilon}\right)_{\varepsilon} \in \mathcal{N}\left[J ; \mathbb{R}^{n}\right]$, e assim $u=v$.

Sejam $K \subset \subset J$ e $r \in \mathbb{N}$. Considere $a, b \in \mathbb{R}$ e $T \in \mathbb{N}^{*}$ tais que

$$
\left.K \cup\left\{t_{0}\right\} \subset\right] a, b[\subset[a, b] \subset \subset J \text { e }[a, b] \subset[-T, T]
$$


De $\left(\nabla_{x} F_{\varepsilon}\right)_{\varepsilon}$ ser $L^{\infty}$ - $\ln$-tipo em $\widetilde{\mathcal{E}}_{\tau}\left[J \times \mathbb{R}^{n} ; \mathbb{R}^{n^{2}}\right]$, existem $c_{1}>0$ e $\eta_{1} \in \mathbf{I}$, tais que

$$
\left|\nabla_{x} F_{\varepsilon}(t, y)\right| \leq c_{1}|\ln \varepsilon|, \quad \forall(\varepsilon, t, y) \in \mathbf{I}_{\eta_{1}} \times[a, b] \times \mathbb{R}^{n}
$$

Seja $q=r+2 T n c_{1}$.

Então, de $\left(n_{\varepsilon}\right)_{\varepsilon} \in \mathcal{N}\left[J ; \mathbb{R}^{n}\right]$ e $\left(m_{\varepsilon}\right)_{\varepsilon} \in(\mathcal{N}(\mathbb{R}))^{n}$, existem $c_{2}>c_{1}$ e $\eta_{2} \in \mathbf{I} \operatorname{com} \eta_{2}<\eta_{1}$ tais que

$$
\begin{gathered}
\left|m_{\varepsilon}\right| \leq c_{2} \varepsilon^{q}, \quad \forall \varepsilon \in \mathbf{I}_{\eta_{2}} \\
\left|n_{\varepsilon}(t)\right| \leq c_{2} \varepsilon^{q}, \quad \forall(\varepsilon, t) \in \mathbf{I}_{\eta_{2}} \times[a, b] .
\end{gathered}
$$

Notemos ainda que

$$
\int_{t_{0}}^{t}\left(F_{\varepsilon}\left(s, u_{\varepsilon}(s)\right)-F_{\varepsilon}\left(s, v_{\varepsilon}(s)\right)+n_{\varepsilon}(s)\right) d s=\left(u_{\varepsilon}(t)-v_{\varepsilon}(t)\right)-\left(u_{\varepsilon}\left(t_{0}\right)-v_{\varepsilon}\left(t_{0}\right)\right),
$$

e assim

$$
u_{\varepsilon}(t)-v_{\varepsilon}(t)=m_{\varepsilon}+\int_{t_{0}}^{t}\left(F_{\varepsilon}\left(s, u_{\varepsilon}(s)\right)-F_{\varepsilon}\left(s, v_{\varepsilon}(s)\right)+n_{\varepsilon}(s)\right) d s,
$$

para todo $(\varepsilon, t) \in \mathbf{I} \times J$.

Suponhamos $\quad\left(F_{\varepsilon}\right)_{\varepsilon}:=\left(\left(F_{1_{\varepsilon}}, \cdots, F_{n_{\varepsilon}}\right)\right)_{\varepsilon}, \quad\left(u_{\varepsilon}\right)_{\varepsilon}:=\left(\left(u_{1_{\varepsilon}}, \cdots, u_{n_{\varepsilon}}\right)\right)_{\varepsilon}$, $\left(n_{\varepsilon}\right)_{\varepsilon}:=\left(\left(n_{1_{\varepsilon}}, \cdots, n_{n_{\varepsilon}}\right)\right)_{\varepsilon} \quad$ e $\quad\left(m_{\varepsilon}\right)_{\varepsilon}:=\left(\left(m_{1_{\varepsilon}}, \cdots, m_{n_{\varepsilon}}\right)\right)_{\varepsilon}$.

Fixemos $1 \leq i \leq n$.

Para facilitar a escrita considere $\left(G_{\varepsilon}\right)_{\varepsilon}:=\left(F_{i_{\varepsilon}}\right)_{\varepsilon}$. Então, de (3.33), (3.34), (3.35) e (3.36), temos que

$$
\begin{gathered}
\left|\nabla_{x} G_{\varepsilon}(t, y)\right| \leq c_{1}|\ln \varepsilon|, \quad \forall(\varepsilon, t, y) \in \mathbf{I}_{\eta_{1}} \times[a, b] \times \mathbb{R}^{n} \\
\left|m_{i_{\varepsilon}}\right| \leq c_{2} \varepsilon^{q}, \quad \forall \varepsilon \in \mathbf{I}_{\eta_{2}} \\
\left|n_{i_{\varepsilon}}(t)\right| \leq c_{2} \varepsilon^{q}, \quad \forall(\varepsilon, t) \in \mathbf{I}_{\eta_{2}} \times[a, b] \\
u_{i_{\varepsilon}}(t)-v_{i_{\varepsilon}}(t)=m_{i_{\varepsilon}}+\int_{t_{0}}^{t}\left(G_{\varepsilon}\left(s, u_{\varepsilon}(s)\right)-G_{\varepsilon}\left(s, v_{\varepsilon}(s)\right)+n_{i_{\varepsilon}}(s)\right) d s, \quad \forall(\varepsilon, t) \in \mathbf{I}_{\eta_{2}} \times[a, b]
\end{gathered}
$$

Fixemos $(\varepsilon, t) \in \mathbf{I}_{\eta_{2}} \times[a, b]$. De (3.38), (3.39) e (3.40) temos que 


$$
\begin{aligned}
\left|u_{i_{\varepsilon}}(t)-v_{i_{\varepsilon}}(t)\right| & =\left|m_{i_{\varepsilon}}+\int_{t_{0}}^{t}\left(G_{\varepsilon}\left(s, u_{\varepsilon}(s)\right)-G_{\varepsilon}\left(s, v_{\varepsilon}(s)\right)+n_{i_{\varepsilon}}(s)\right) d s\right| \\
& \leq\left|m_{i_{\varepsilon}}\right|+\left|\int_{t_{0}}^{t}\right| G_{\varepsilon}\left(s, u_{\varepsilon}(s)\right)-G_{\varepsilon}\left(s, v_{\varepsilon}(s)\right)|d s|+\left|\int_{t_{0}}^{t}\right| n_{i_{\varepsilon}}(s)|d s| \\
& \leq c_{2} \varepsilon^{q}+\left|\int_{t_{0}}^{t} c_{2} \varepsilon^{q} d s\right|+\left|\int_{t_{0}}^{t}\right| G_{\varepsilon}\left(s, u_{\varepsilon}(s)\right)-G_{\varepsilon}\left(s, v_{\varepsilon}(s)\right)|d s| \\
& \leq c_{2} \varepsilon^{q}+c_{2}\left|t-t_{0}\right| \varepsilon^{q}+\left|\int_{t_{0}}^{t}\right| G_{\varepsilon}\left(s, u_{\varepsilon}(s)\right)-G_{\varepsilon}\left(s, v_{\varepsilon}(s)\right)|d s| \\
& \leq c_{2} \varepsilon^{q}+2 T c_{2} \varepsilon^{q}+\left|\int_{t_{0}}^{t}\right| G_{\varepsilon}\left(s, u_{\varepsilon}(s)\right)-G_{\varepsilon}\left(s, v_{\varepsilon}(s)\right)|d s| \\
& \leq c_{2}(1+2 T) \varepsilon^{q}+\left|\int_{t_{0}}^{t}\right| G_{\varepsilon}\left(s, u_{\varepsilon}(s)\right)-G_{\varepsilon}\left(s, v_{\varepsilon}(s)\right)|d s| .
\end{aligned}
$$

Usando o Teorema do Valor Médio na segunda parcela da última desigualdade acima temos

$$
\left|u_{i_{\varepsilon}}(t)-v_{i_{\varepsilon}}(t)\right| \leq c_{2}(1+2 T) \varepsilon^{q}+\left|\int_{t_{0}}^{t}\right| u_{\varepsilon}(s)-v_{\varepsilon}(s)|| \nabla_{x} G_{\varepsilon}\left(s, w_{\varepsilon}(s)\right)|d s|,
$$

onde $w_{\varepsilon}(s):=u_{\varepsilon}(s)+\sigma_{s}\left(u_{\varepsilon}(s)-v_{\varepsilon}(s)\right)$ para algum $\sigma_{s} \in[0,1]$.

Como $(\varepsilon, t) \in \mathbf{I}_{\eta_{2}} \times[a, b]$ e $1 \leq i \leq n$ são arbitrários temos, de (3.37), que $\left|u_{i_{\varepsilon}}(t)-v_{i_{\varepsilon}}(t)\right| \leq c_{3} \varepsilon^{q}+\left|\int_{t_{0}}^{t}\right| u_{\varepsilon}(s)-v_{\varepsilon}(s)\left|c_{1}\right| \ln \varepsilon|d s|, \quad \forall(\varepsilon, t) \in \mathbf{I}_{\eta_{2}} \times[a, b], \forall 1 \leq i \leq n$, onde $c_{3}:=c_{2}(1+2 T)$. Logo

$$
\left|u_{\varepsilon}(t)-v_{\varepsilon}(t)\right| \leq n c_{3} \varepsilon^{q}+\left|\int_{t_{0}}^{t} n c_{1}\right| \ln \varepsilon|| u_{\varepsilon}(s)-v_{\varepsilon}(s)|d s|, \quad \forall(\varepsilon, t) \in \mathbf{I}_{\eta_{2}} \times[a, b],
$$

Assim, pelo Lema 3.1.16, tem-se, para todo $(\varepsilon, t) \in \mathbf{I}_{\eta_{2}} \times[a, b]$, que

$$
\begin{aligned}
\left|u_{\varepsilon}(t)-v_{\varepsilon}(t)\right| & \leq n c_{3} \varepsilon^{q} \exp \left(\left|\int_{t_{0}}^{t} n c_{1}\right| \ln \varepsilon|d s|\right) \\
& \leq n c_{3} \varepsilon^{q} \exp \left(-2 T n c_{1} \ln \varepsilon\right) \\
& \leq n c_{3} \varepsilon^{q} \exp \left(\ln \varepsilon^{-2 T n c_{1}}\right) \\
& \leq n c_{3} \varepsilon^{q-2 T n c_{1}}=c \varepsilon^{r}
\end{aligned}
$$

onde $c:=n c_{3}$. Portanto $\left(u_{\varepsilon}-v_{\varepsilon}\right)_{\varepsilon}$ satisfaz as hipóteses do Teorema de Nulidade em $\mathcal{G}\left(J ; \mathbb{R}^{n}\right)$ (Teorema 1.2.5). Desse fato concluímos que $\left(u_{\varepsilon}-v_{\varepsilon}\right)_{\varepsilon} \in \mathcal{N}\left[J ; \mathbb{R}^{n}\right]$, ou seja, o problema (3.3) admite uma única solução em $\mathcal{G}\left(J ; \mathbb{R}^{n}\right)$, o que prova 1 .. 
Finalmente, suponhamos $F \in \mathcal{G}_{\tau}\left(J \times \mathbb{R}^{n} ; \mathbb{R}^{n}\right)$ e seja $\left(F_{\varepsilon}\right)_{\varepsilon}$ um representante de $F$ tal que $\left(\nabla_{x} F_{\varepsilon}\right)_{\varepsilon}$ é $L^{\infty}$-ln-tipo em $\mathcal{E}_{\tau}\left[\mathbb{R} \times \mathbb{R}^{n} ; \mathbb{R}^{n}\right]$. Da Proposição 1.4 .11 (1.), temos que $\left(F_{\varepsilon}\right)_{\varepsilon} \in \widetilde{\mathcal{E}}_{\tau}\left[\mathbb{R} \times \mathbb{R}^{n} ; \mathbb{R}^{n}\right]$ e, pela Proposição 3.1 .15 (2.), temos que $\left(\nabla_{x} F_{\varepsilon}\right)_{\varepsilon}$ é $L^{\infty}$ - ln-tipo em $\widetilde{\mathcal{E}}_{\tau}\left[\mathbb{R} \times \mathbb{R}^{n} ; \mathbb{R}^{n^{2}}\right]$. Portanto, pela prova do caso anterior, o problema (3.3) admite uma única solução em $\mathcal{G}\left(J ; \mathbb{R}^{n}\right)$, o que prova 2 ..

A exigência de $\nabla_{x} F$ ser de $L^{\infty}$ - ln-tipo em $\widetilde{\mathcal{G}}_{\tau}\left(J \times \mathbb{R}^{n} ; \mathbb{R}^{n^{2}}\right)$ ou em $\mathcal{G}_{\tau}\left(J \times \mathbb{R}^{n} ; \mathbb{R}^{n^{2}}\right)$ no Teorema 3.1.21 é de extrema importância para a obtenção da existência e da unicidade de solução em $\mathcal{G}\left(J ; \mathbb{R}^{n}\right)$. A seguir mostraremos, através de dois exemplos, que a sua ausência pode não garantir a existência de solução e, quando houver solução, essa poderá não ser única.

Exemplo 3.1.22 (Não existência) Seja $F:=\left[\left(F_{\varepsilon}\right)_{\varepsilon}\right] \in \mathcal{G}_{\tau}(\mathbb{R} \times \mathbb{R} ; \mathbb{R})$, onde

$$
F_{\varepsilon}:(t, x) \in \mathbb{R} \times \mathbb{R} \longmapsto \frac{x}{\varepsilon} .
$$

Então $\nabla_{x} F$ não é $L^{\infty}$-ln-tipo em $\mathcal{G}_{\tau}(\mathbb{R} \times \mathbb{R} ; \mathbb{R})$ e o problema

$$
\begin{aligned}
\frac{d u}{d t} & =F \circ(i d, u) \\
u(0) & =i_{R}(1)
\end{aligned}
$$

onde $i_{R}: \mathbb{R} \longrightarrow \overline{\mathbb{R}}$ é a inclusão de $\mathbb{R}$ em $\overline{\mathbb{R}}$, não tem solução em $\mathcal{G}(\mathbb{R} ; \mathbb{R})$.

De fato, considere $\left(g_{\varepsilon}\right)_{\varepsilon}$ o representante de $\nabla_{x} F$ dado por

$$
g_{\varepsilon}(t, x)=\frac{1}{\varepsilon}, \quad \forall(\varepsilon, t, x) \in \mathbf{I} \times \mathbb{R} \times \mathbb{R} .
$$

Então, do Exemplo 3.1.13, temos que $\nabla_{x} F$ não é $L^{\infty}$ - ln-tipo em $\mathcal{G}_{\tau}(\mathbb{R} \times \mathbb{R} ; \mathbb{R})$. Para verificar que $(3.41)$ não tem solução em $\mathcal{G}(\mathbb{R} ; \mathbb{R})$, observe que, se $u=\left[\left(u_{\varepsilon}\right)_{\varepsilon}\right] \in \mathcal{G}(\mathbb{R} ; \mathbb{R})$ for uma solução de (3.41), então existem $\left(n_{\varepsilon}\right)_{\varepsilon} \in \mathcal{N}[\mathbb{R} ; \mathbb{R}]$ e $\left(m_{\varepsilon}\right)_{\varepsilon} \in \mathcal{N}(\mathbb{R})$ tais que

$$
\begin{aligned}
\frac{d u_{\varepsilon}}{d t}(t) & =\frac{1}{\varepsilon} u_{\varepsilon}(t)+n_{\varepsilon}(t), \quad \forall(\varepsilon, t) \in \mathbf{I} \times \mathbb{R} ; \\
u_{\varepsilon}(0) & =1+m_{\varepsilon} ; \quad \forall \varepsilon \in \mathbf{I} .
\end{aligned}
$$


Note que, desses fatos tem-se que

$$
u_{\varepsilon}(t)=\left(1+m_{\varepsilon}\right) \mathrm{e}^{t / \varepsilon}+\int_{0}^{t} n_{\varepsilon}(s) \mathrm{e}^{(t-s) / \varepsilon} d s, \quad \forall(\varepsilon, t) \in \mathbf{I} \times \mathbb{R} .
$$

$\operatorname{De}\left(u_{\varepsilon}\right)_{\varepsilon} \in \mathcal{E}_{M}[\mathbb{R} ; \mathbb{R}],\left(n_{\varepsilon}\right)_{\varepsilon} \in \mathcal{N}[\mathbb{R} ; \mathbb{R}]$ e $\left(m_{\varepsilon}\right)_{\varepsilon} \in \mathcal{N}(\mathbb{R})$, existem $N \in \mathbb{N}, c>0$ e $\eta \in \mathbf{I}$ tais que

$$
\begin{gathered}
\left|u_{\varepsilon}(1)\right| \leq c \varepsilon^{-N}, \quad \forall \varepsilon \in \mathbf{I}_{\eta} \\
\left|n_{\varepsilon}(1)\right| \leq c \varepsilon^{2}, \quad \forall \varepsilon \in \mathbf{I}_{\eta} \\
\left|m_{\varepsilon}\right| \leq c \varepsilon, \quad \forall \varepsilon \in \mathbf{I}_{\eta}
\end{gathered}
$$

e de (3.42),

$$
\left|u_{\varepsilon}(1)\right|=\left|\left(1+m_{\varepsilon}\right) \mathrm{e}^{1 / \varepsilon}+\int_{0}^{1} n_{\varepsilon}(s) \mathrm{e}^{(1-s) / \varepsilon} d s\right|, \quad \forall \varepsilon \in \mathbf{I}_{\eta} .
$$

Assim, para $\varepsilon<\min \left\{\frac{1}{4 c}, \eta\right\}$, tem-se

$$
\begin{aligned}
c \varepsilon^{-N} \geq\left|u_{\varepsilon}(1)\right| & \geq\left|\left(1+m_{\varepsilon}\right) \mathrm{e}^{1 / \varepsilon}\right|-\left|\int_{0}^{1} n_{\varepsilon}(s) \mathrm{e}^{(1-s) / \varepsilon} d s\right| \\
& \geq|1-| m_{\varepsilon}|| \mathrm{e}^{1 / \varepsilon}-\int_{0}^{1}\left|n_{\varepsilon}(s)\right| \mathrm{e}^{(1-s) / \varepsilon} d s \\
& \geq \frac{3}{4} \mathrm{e}^{1 / \varepsilon}-\int_{0}^{1} c \varepsilon^{2} \mathrm{e}^{(1-s) / \varepsilon} d s \\
& \geq \frac{3}{4} \mathrm{e}^{1 / \varepsilon}-\int_{0}^{1}(c \varepsilon)\left(\varepsilon \mathrm{e}^{1 / \varepsilon}\right) d s \\
& \geq \frac{3}{4} \mathrm{e}^{1 / \varepsilon}-\frac{1}{4} \mathrm{e}^{1 / \varepsilon} \\
& \geq \frac{1}{2} \mathrm{e}^{1 / \varepsilon},
\end{aligned}
$$

o que é um absurdo, pois $\lim _{\varepsilon \downarrow 0}\left(c \varepsilon^{-N} \mathrm{e}^{-1 / \varepsilon}\right)=0$.

É importante observar o seguinte.

Observação 3.1.23 O Exemplo 3.1.22 nos mostra que a condição de $\nabla_{x} F$ ser $L^{\infty}$-tipo, apesar de garantir (3.13), não garante a existência de uma solução generalizada para o problema (3.3). 
Exemplo 3.1.24 (Não unicidade) Seja $F:=\left[\left(F_{\varepsilon}\right)_{\varepsilon}\right] \in \mathcal{G}_{\tau}(\mathbb{R} \times \mathbb{R} ; \mathbb{R})$, onde

$$
F_{\varepsilon}:(t, x) \in \mathbb{R} \times \mathbb{R} \longmapsto \tanh \frac{x}{\varepsilon} .
$$

Então $\nabla_{x} F$ não é $L^{\infty}$-ln-tipo em $\mathcal{G}_{\tau}(\mathbb{R} \times \mathbb{R} ; \mathbb{R})$ e o problema

$$
\begin{aligned}
\frac{d u}{d t} & =F \circ(i d, u) \\
u(0) & =i_{R}(0)
\end{aligned}
$$

tem mais que uma solução em $\mathcal{G}(\mathbb{R} ; \mathbb{R})$.

De fato, é claro que a função nula é uma solução. Para obtermos outra solução observe que $\left(\varepsilon \mathrm{e}^{-1 / \varepsilon}\right) \in \mathcal{N}(\mathbb{R})$ e que, se, para cada $\varepsilon \in \mathbf{I}$, resolvermos a equação

$$
\frac{d u_{\varepsilon}}{d t}(t)=\tanh \left(\frac{u_{\varepsilon}(t)}{\varepsilon}\right), \quad t \in \mathbb{R},
$$

com a condição inicial $u_{\varepsilon}(0)=\varepsilon \mathrm{e}^{-1 / \varepsilon}$, obteremos a solução

$$
u_{\varepsilon}(t):=\varepsilon \operatorname{Arcsenh}\left(\mathrm{e}^{t / \varepsilon} \operatorname{senh}\left(\mathrm{e}^{-1 / \varepsilon}\right)\right), \quad t \in \mathbb{R} .
$$

Note ainda que, para $\varepsilon \in \mathbf{I}$, temos

$$
u_{\varepsilon}(t)<\varepsilon \mathrm{e}^{-1}<1, \quad \forall t<0,
$$

pois senh é crescente em $\mathbb{R}$ e $\operatorname{senh}(0)=0$;

$$
u_{\varepsilon}(t)<1+t, \quad \forall t>0,
$$

pois $u_{\varepsilon}(0)=\varepsilon \mathrm{e}^{-1 / \varepsilon}<1 \mathrm{e} \frac{d u_{\varepsilon}}{d t}(t)<1$, para $t>0$. Dessa forma, segue que

$$
\left|u_{\varepsilon}(t)\right| \leq 1+|t|, \quad \forall(\varepsilon, t) \in \mathbf{I} \times \mathbb{R},
$$

e assim, dado $T \in \mathbb{N}^{*}$ tem-se que

$$
\left|u_{\varepsilon}(t)\right| \leq 1+T, \quad \forall(\varepsilon, t) \in \mathbf{I} \times[-T, T] .
$$

Usando que tanh é limitada, $(\tanh )^{\prime}=(\text { sech })^{2}$, que sech é limitada e $(\text { sech })^{\prime}=$ sech tanh, é fácil verificar que, para $m \in \mathbb{N}^{*}$, existe $c>0$ tal que

$$
\left|\frac{d^{m} u_{\varepsilon}}{d t^{m}}(t)\right| \leq \frac{c}{\varepsilon^{m}}, \quad \forall(\varepsilon, t) \in \mathbf{I} \times \mathbb{R}
$$


Portanto, $\left(u_{\varepsilon}\right)_{\varepsilon} \in \mathcal{E}_{M}[\mathbb{R} ; \mathbb{R}]$. Logo $u=\left(u_{\varepsilon}\right)_{\varepsilon}+\mathcal{N}[\mathbb{R} ; \mathbb{R}]$ é uma solução do problema proposto. De senh $(t)>t$, para todo $t>0$ e Arc senh ser crescente, temos que

$$
\left|u_{\varepsilon}(1)\right|=\varepsilon \operatorname{Arcsenh}\left(\mathrm{e}^{1 / \varepsilon} \operatorname{senh}\left(\mathrm{e}^{-1 / \varepsilon}\right)\right) \geq \varepsilon \operatorname{Arcsenh}\left(\mathrm{e}^{1 / \varepsilon} \mathrm{e}^{-1 / \varepsilon}\right)=\varepsilon \operatorname{Arcsenh}(1)>0
$$

$\operatorname{Logo},\left(u_{\varepsilon}\right)_{\varepsilon} \notin \mathcal{N}[\mathbb{R} ; \mathbb{R}]$, pois, se pertencesse a $\mathcal{N}[\mathbb{R} ; \mathbb{R}]$, existiriam $c>0$ e $\eta \in \mathbf{I}$ tais que

$$
\left|u_{\varepsilon}(1)\right| \leq c \varepsilon^{2}, \quad \forall \varepsilon \in \mathbf{I}_{\eta},
$$

o que levaria a $c \varepsilon \geq \operatorname{Arc} \operatorname{senh}(1)>0$, para todo $\varepsilon \in \mathbf{I}_{\eta}$, o que é um absurdo. Portanto, $u:=\left[\left(u_{\varepsilon}\right)_{\varepsilon}\right] \in \mathcal{G}(\mathbb{R} ; \mathbb{R})$ é uma solução do problema proposto que é diferente da solução nula. Finalmente, note que $\left(g_{\varepsilon}\right)_{\varepsilon}$ dado por

$$
g_{\varepsilon}(t, x)=\frac{1}{\varepsilon}\left[\operatorname{sech}\left(\frac{x}{\varepsilon}\right)\right]^{2}, \quad \forall(\varepsilon, t, x) \in \mathbf{I} \times \mathbb{R} \times \mathbb{R}
$$

é um representante de $\nabla_{x} F$ e $\nabla_{x} F$ não é $L^{\infty}$ - ln-tipo em $\mathcal{G}_{\tau}(\mathbb{R} \times \mathbb{R} ; \mathbb{R})($ Exemplo 3.1.14).

Dando continuidade ao nosso estudo, observe que, se $G \in \mathscr{C}^{\infty}\left(\mathbb{R} \times \mathbb{R}^{n} ; \mathbb{R}^{n}\right)$ é tal que $\nabla_{x} G$ é limitado, então, pelo comentário feito antes de (3.13), o problema $\frac{d w}{d t}(t)=G(t, w(t))$, com condição inicial $w\left(t_{0}\right)=y_{0} \in \mathbb{R}^{n}$, tem uma única solução $w \in \mathscr{C}^{\infty}\left(\mathbb{R} ; \mathbb{R}^{n}\right)$. Pode ser que $(G)_{\varepsilon}$ não pertença a $\mathcal{E}_{\tau}\left[\mathbb{R} \times \mathbb{R}^{n} ; \mathbb{R}^{n}\right]$, mas, se $G$ pertencer a $\mathcal{O}_{M}\left(\mathbb{R} \times \mathbb{R}^{n} ; \mathbb{R}^{n}\right)$ (Definição 1.3 .16$)$ é claro que $(G)_{\varepsilon} \in \mathcal{E}_{\tau}\left[\mathbb{R} \times \mathbb{R}^{n} ; \mathbb{R}^{n}\right]$. Assim, se considerarmos $G \in \mathcal{O}_{M}\left(\mathbb{R} \times \mathbb{R}^{n} ; \mathbb{R}^{n}\right)$ e $i_{R^{n}}$ o homomorfismo injetor definido por

$$
\begin{array}{ll}
i_{R^{n}}: \mathbb{R}^{n} & \longrightarrow(\overline{\mathbb{R}})^{n} \\
\left(x_{1}, \cdots, x_{n}\right) & \longmapsto\left(x_{1}+\mathcal{N}(\mathbb{R}), \cdots, x_{n}+\mathcal{N}(\mathbb{R})\right)
\end{array}
$$

temos que o problema (3.3), para $F:=i_{\mathcal{O}_{M}}(G)$ e $x_{0}:=i_{R^{n}}\left(y_{0}\right)$, admite como solução $i_{\mathscr{C} \infty\left(R ; R^{n}\right)}(w)$. Nesse caso, é interessante saber se há uma outra solução além de $i_{\mathscr{C} \infty\left(R ; R^{n}\right)}(w)$. Como resposta temos o seguinte resultado:

Proposição 3.1.25 Seja $G \in \mathcal{O}_{M}\left(\mathbb{R} \times \mathbb{R}^{n} ; \mathbb{R}^{n}\right)$ tal que $\nabla_{x} G$ é limitado. Então o problema (3.3), para $F:=i_{\mathcal{O}_{M}}(G)$ e $x_{0}:=i_{R^{n}}\left(y_{0}\right)$, onde $y_{0} \in \mathbb{R}$, admite uma única solução 
u em $\mathcal{G}\left(\mathbb{R} ; \mathbb{R}^{n}\right)$. Além disso, essa solução é $i_{\mathscr{C} \infty}\left(\Omega ; R^{m}\right)(w)$, onde w é o único elemento de $\mathscr{C}^{\infty}\left(\mathbb{R} ; \mathbb{R}^{n}\right)$ tal que $\frac{d w}{d t}(t)=G(t, w(t))$, para todo $t \in \mathbb{R}$, e $w\left(t_{0}\right)=y_{0}$.

Demonstração: Da teoria clássica das Equações Diferenciais Ordinárias (comentário acima de $(3.13))$, existe uma única $w \in \mathscr{C}^{\infty}\left(\mathbb{R} ; \mathbb{R}^{n}\right)$ tal que

$$
\begin{aligned}
\frac{d w}{d t} & =G(t, w(t)) \\
w\left(t_{0}\right) & =y_{0}
\end{aligned}
$$

Seja $u:=i_{\mathscr{C} \infty}\left(R ; R^{n}\right)(w)$. É claro que $u \in \mathcal{G}\left(\mathbb{R} ; \mathbb{R}^{n}\right)$ é uma solução de (3.3), para $F=i_{\mathcal{O}_{M}}(G)$ e $x_{0}=i_{R^{n}}\left(y_{0}\right)$.

Note que $\nabla_{x} F$ é $L^{\infty}$ - ln-tipo em $\mathcal{G}_{\tau}\left(\mathbb{R} \times \mathbb{R}^{n} ; \mathbb{R}^{n}\right)$, pois $\nabla_{x} G$ é limitada. Assim, pelo Teorema 3.1.21 (2.), o problema (3.3) admite uma única solução em $\mathcal{G}\left(\mathbb{R} ; \mathbb{R}^{n}\right)$. Logo, essa única solução é $i_{\mathscr{C} \infty}\left(R ; R^{n}\right)(w)$.

Na Proposição 3.1.26 apresentaremos condições de existência de solução para o problema (3.3) sem a condição de $\nabla_{x} F \quad$ ser $\quad L^{\infty}$-ln-tipo em $\quad \mathcal{G}_{\tau}\left(\mathbb{R} \times \mathbb{R}^{n} ; \mathbb{R}^{n}\right.$ ) (ou em $\left.\widetilde{\mathcal{G}}_{\tau}\left(\mathbb{R} \times \mathbb{R}^{n} ; \mathbb{R}^{n}\right)\right)$. Para isso, notemos que, se $J$ é um intervalo aberto de $\mathbb{R}$, $f \in \mathscr{C}^{\infty}\left(J \times \mathbb{R}^{n} ; \mathbb{R}^{n}\right)$ é limitada e $g \in \mathscr{C}^{\infty}\left(J \times \mathbb{R}^{n} ; \mathbb{R}^{n+1}\right)$ é definida por

$$
g(s, x)=(1, f(s, x)), \quad \forall(s, x) \in J \times \mathbb{R}^{n},
$$

então, a partir do Teorema 1.1.3 de [FS], dados $t_{0} \in J$ e $x_{0} \in \mathbb{R}^{n}$, existe uma solução $v=\left(v_{0}, v_{1}, \cdots, v_{n}\right) \in \mathscr{C}^{\infty}\left(J ; \mathbb{R}^{n+1}\right)$ tal que

$$
\frac{d v}{d t}(t)=g(v(t)), \quad \forall t \in J \quad \text { e } \quad v\left(t_{0}\right)=\left(t_{0}, x_{0}\right)
$$

Assim a função $u:=\left(v_{1}, \cdots, v_{n}\right) \in \mathscr{C}^{\infty}\left(J ; \mathbb{R}^{n}\right)$ satisfaz

$$
\frac{d u}{d t}=f(t, u(t)), \quad \forall t \in J \quad \text { e } \quad u\left(t_{0}\right)=x_{0}
$$


Proposição 3.1.26 Sejam $J$ um intervalo aberto de $\mathbb{R}$ e $F \in \mathcal{G}_{\tau}\left(J \times \mathbb{R}^{n} ; \mathbb{R}^{n}\right)$ (respectivamente $\left.F \in \widetilde{\mathcal{G}}_{\tau}\left(J \times \mathbb{R}^{n} ; \mathbb{R}^{n}\right)\right)$. Se $\quad F \quad$ é $L^{\infty}$-tipo em $\quad \mathcal{G}_{\tau}\left(J \times \mathbb{R}^{n} ; \mathbb{R}^{n}\right) \quad$ (resp. em $\left.\widetilde{\mathcal{G}}_{\tau}\left(J \times \mathbb{R}^{n} ; \mathbb{R}^{n}\right)\right)$, então o problema $(3.3)$ tem uma solução $u \in \mathcal{G}\left(J ; \mathbb{R}^{n}\right)$.

Demonstração: Seja $\left(F_{\varepsilon}\right)_{\varepsilon}$ um representante de $F$ tal que $\left(F_{\varepsilon}\right)_{\varepsilon}$ é $L^{\infty}$-tipo em $\mathcal{E}_{\tau}\left[J \times \mathbb{R}^{n} ; \mathbb{R}^{n}\right]$ ( respectivamente em $\widetilde{\mathcal{E}}_{\tau}\left[J \times \mathbb{R}^{n} ; \mathbb{R}^{n}\right]$ ). Notemos que, se $\left(F_{\varepsilon}\right)_{\varepsilon} \in \mathcal{E}_{\tau}\left[J \times \mathbb{R}^{n} ; \mathbb{R}^{n}\right]$ e $\left(F_{\varepsilon}\right)_{\varepsilon} \in \mathcal{E}_{\tau, L^{\infty}}\left[J \times \mathbb{R}^{n} ; \mathbb{R}^{n}\right]$ então, pelas Proposição 1.4.11 (1.) e Proposição 3.1.15), temos que $\left(F_{\varepsilon}\right)_{\varepsilon} \in \widetilde{\mathcal{E}}_{\tau}\left[J \times \mathbb{R}^{n} ; \mathbb{R}^{n}\right]$ e $\left(F_{\varepsilon}\right)_{\varepsilon} \in \widetilde{\mathcal{E}}_{\tau, L^{\infty}}\left[J \times \mathbb{R}^{n} ; \mathbb{R}^{n}\right]$. Logo, basta mostrar o caso em que $\left(F_{\varepsilon}\right)_{\varepsilon} \in \widetilde{\mathcal{E}}_{\tau}\left[J \times \mathbb{R}^{n} ; \mathbb{R}^{n}\right]$ e $\left(F_{\varepsilon}\right)_{\varepsilon} \in \widetilde{\mathcal{E}}_{\tau, L \infty}\left[J \times \mathbb{R}^{n} ; \mathbb{R}^{n}\right]$.

Seja $\left(x_{0_{\varepsilon}}\right)_{\varepsilon} \in\left(\mathcal{E}_{M}(\mathbb{R})\right)^{n}$ um representante de $x_{0}$.

Fixemos $\varepsilon \in$ I. Como $F_{\varepsilon}$ é limitada, existe, pelo comentário feito antes desta proposição, $u_{\varepsilon} \in\left(\mathscr{C}^{\infty}\left(J ; \mathbb{R}^{n}\right)\right)^{\mathbf{I}}$ tal que

$$
\begin{aligned}
\frac{d u_{\varepsilon}}{d t}(t) & =F_{\varepsilon}\left(t, u_{\varepsilon}(t)\right), \quad \forall t \in J ; \\
u_{\varepsilon}\left(t_{0}\right) & =x_{0_{\varepsilon}}
\end{aligned}
$$

e assim

$$
u_{\varepsilon}(t)=x_{0_{\varepsilon}}+\int_{t_{0}}^{t} F_{\varepsilon}\left(s, u_{\varepsilon}(s)\right) d s, \quad \forall t \in J
$$

Mostraremos que $\left(u_{\varepsilon}\right)_{\varepsilon} \in \mathcal{E}_{M}\left[\mathbb{R} ; \mathbb{R}^{n}\right]$, e dessa forma, $u=\left[\left(u_{\varepsilon}\right)_{\varepsilon}\right] \in \mathcal{G}\left(J ; \mathbb{R}^{n}\right)$ será uma solução de (3.3).

Seja $K \subset \subset J$. Considere $a, b \in \mathbb{R}$ e $T \in \mathbb{N}^{*}$ tais que

$$
\left.K \cup\left\{t_{0}\right\} \subset\right] a, b[\subset[a, b] \subset J \text { e }[a, b] \subset[-T, T]
$$

Provaremos que existem $N \in \mathbb{N}, c>0$ e $\eta \in \mathbf{I}$ tais que

$$
\left|u_{\varepsilon}(t)\right| \leq c \varepsilon^{-N}, \quad \forall(\varepsilon, t) \in \mathbf{I}_{\eta} \times[a, b]
$$

$\operatorname{De}\left(x_{0_{\varepsilon}}\right)_{\varepsilon} \in\left(\mathcal{E}_{M}(\mathbb{R})\right)^{n}$ e $\left(F_{\varepsilon}\right)_{\varepsilon} \in \widetilde{\mathcal{E}}_{\tau, L^{\infty}}\left[J \times \mathbb{R}^{n} ; \mathbb{R}^{n}\right]$, existem $N \in \mathbb{N}, c_{1}>0$ e $\eta \in \mathbf{I}$ tais que

$$
\begin{gathered}
\left|x_{0_{\varepsilon}}\right| \leq c_{1} \varepsilon^{-N}, \quad \forall \varepsilon \in \mathbf{I}_{\eta} \\
\left|F_{\varepsilon}(t, y)\right| \leq c_{1} \varepsilon^{-N}, \quad \forall(\varepsilon, t, y) \in \mathbf{I}_{\eta} \times[a, b] \times \mathbb{R}^{n} .
\end{gathered}
$$


De (3.47), (3.48) e (3.49) temos que

$$
\begin{aligned}
\left|u_{\varepsilon}(t)\right| & \leq\left|x_{0_{\varepsilon}}\right|+\left|\int_{t_{0}}^{t}\right| F_{\varepsilon}\left(s, u_{\varepsilon}(s)\right)|d s| \\
& \leq c_{1} \varepsilon^{-N}+\left|\int_{t_{0}}^{t} c_{1} \varepsilon^{-N} d s\right| \\
& \leq c_{1} \varepsilon^{-N}+\left|\left(|t|+\left|t_{0}\right|\right) c_{1} \varepsilon^{-N}\right| \\
& \leq c_{1} \varepsilon^{-N}+2 T c_{1} \varepsilon^{-N} \\
& \leq c_{1}(1+2 T) \varepsilon^{-N},
\end{aligned}
$$

para todo $(\varepsilon, t) \in \mathbf{I}_{\eta} \times[a, b]$.

Seja $c:=c_{1}(1+2 T)$, então

$$
\left|u_{\varepsilon}(t)\right| \leq c \varepsilon^{-N}, \quad \forall(\varepsilon, t) \in \mathbf{I}_{\eta} \times[a, b]
$$

e assim, da Proposição 3.1.17, tem-se que $\left(u_{\varepsilon}\right)_{\varepsilon} \in \mathcal{E}_{M}\left[J ; \mathbb{R}^{n}\right]$.

É importante notar o seguinte.

Observação 3.1.27 Nas condições da Proposição 3.1.26 não se pode garantir a unicidade de solução.

De fato, basta observar que a função $F$ do Exemplo 3.1.24 é $L^{\infty}$-tipo em $\mathcal{G}_{\tau}(\mathbb{R} ; \mathbb{R})$, pois $\left(\tanh \frac{x}{\varepsilon}\right)_{\varepsilon}$ é um representante de $F$ e $-1<\tanh \frac{x}{\varepsilon}<1$, para todo $(\varepsilon, x) \in \mathbf{I} \times \mathbb{R}$.

Decorre da prova do Teorema 3.1.21 que, sob as hipóteses lá estabelecidas, para cada $\varepsilon$ fixado, a solução clássica do problema

$$
\begin{aligned}
\frac{d u_{\varepsilon}}{d t}(t) & =F_{\varepsilon}\left(t, u_{\varepsilon}(t)\right), \quad \forall t \in J \\
u_{\varepsilon}\left(t_{0}\right) & =x_{0_{\varepsilon}}
\end{aligned}
$$

nos permite obter uma solução em $\mathcal{G}\left(J ; \mathbb{R}^{n}\right)$ que é dada pela classe de $\left(u_{\varepsilon}\right)_{\varepsilon}$. Se $\left(u_{\varepsilon}\right)_{\varepsilon}$ for moderada temperada é claro que sua classe em $\mathcal{G}_{\tau}\left(J ; \mathbb{R}^{n}\right)$ é uma solução de $(3.3)$ em 
$\mathcal{G}_{\tau}\left(J ; \mathbb{R}^{n}\right)$. Contudo, $\left(u_{\varepsilon}\right)_{\varepsilon}$ pode não pertencer a $\mathcal{E}_{\tau}\left[J ; \mathbb{R}^{n}\right]$, como mostra o próximo exemplo. Esse fato era de se esperar, visto que em $\mathcal{E}_{\tau}\left[J ; \mathbb{R}^{n}\right]$ não se trabalha com compactos. Para garantir que $\left(u_{\varepsilon}\right)_{\varepsilon}$ seja um elemento moderado temperado é razoável procurar restringir $u_{\varepsilon}$ a um intervalo $L$ aberto limitado de $J$, e assim procurar solução em $\mathcal{G}_{\tau}\left(L ; \mathbb{R}^{n}\right)$ para o problema (3.3). O Teorema 3.1.29 fornece condições para que a família gerada pela solução clássica pertença a $\mathcal{E}_{\tau}\left[J ; \mathbb{R}^{n}\right]$.

Exemplo 3.1.28 Seja $F=\left[\left(F_{\varepsilon}\right)_{\varepsilon}\right] \in \mathcal{G}_{\tau}(\mathbb{R} \times \mathbb{R} ; \mathbb{R})$, onde

$$
F_{\varepsilon}:(t, x) \in \mathbb{R} \times \mathbb{R} \longmapsto x .
$$

Então $\nabla_{x} F$ é $L^{\infty}$-ln-tipo em $\mathcal{G}_{\tau}(\mathbb{R} \times \mathbb{R} ; \mathbb{R})$ e o problema

$$
\begin{aligned}
\frac{d u}{d t} & =F \circ(i d, u) \\
u(0) & =i_{R}(1)
\end{aligned}
$$

tem uma única solução generalizada que é a classe de $\left(u_{\varepsilon}\right)_{\varepsilon}$ em $\mathcal{G}(\mathbb{R} ; \mathbb{R})$, onde, para $\varepsilon \in \mathbf{I}$, $u_{\varepsilon}$ é a solução clássica de $\frac{d u_{\varepsilon}}{d t}(t)=F(t, u(t))$, para $t \in \mathbb{R}$ e $u_{\varepsilon}(0)=1$. Contudo, $\left(u_{\varepsilon}\right)_{\varepsilon}$ não é um elemento moderado temperado.

De fato, notemos que $\left(\nabla_{x} F_{\varepsilon}\right)_{\varepsilon}=(1)_{\varepsilon}$, e portanto, $\nabla_{x} F$ é $L^{\infty}$-ln-tipo em $\mathcal{G}_{\tau}(\mathbb{R} \times \mathbb{R} ; \mathbb{R})$. Resolvendo, para cada $\varepsilon \in \mathbf{I}$, a equação $\frac{d u_{\varepsilon}}{d t}(t)=F_{\varepsilon}\left(t, u_{\varepsilon}(t)\right)=u_{\varepsilon}(t)$, para todo $(\varepsilon, t) \in \mathbf{I} \times \mathbb{R}$ com a condição inicial $u_{\varepsilon}(0)=1$, temos que $u_{\varepsilon}(t)=\mathrm{e}^{t}$ é uma solução.

Seja $G \in \mathcal{O}_{M}(\mathbb{R} \times \mathbb{R} ; \mathbb{R})$ definida por $G(t, x)=x$. Como $G \in \mathcal{O}_{M}(\mathbb{R} \times \mathbb{R} ; \mathbb{R})$ e $\nabla_{x} G$ é limitado, temos, pela Proposição 3.1.25, que a classe de $\left(u_{\varepsilon}\right)_{\varepsilon}$ é a única solução de (3.50) em $\mathcal{G}(\mathbb{R} ; \mathbb{R})$. Obviamente $\left(u_{\varepsilon}\right)_{\varepsilon} \notin \mathcal{E}_{\tau}[\mathbb{R} ; \mathbb{R}]$, pois dado qualquer $(\varepsilon, c, N) \in \mathbf{I} \times \mathbb{R}{ }_{+} \times \mathbb{N}$, existe $t \in \mathbb{R}$, tal que

$$
\left|u_{\varepsilon}(t)\right|=\left|e^{t}\right| \geq c(1+|t|)^{N} \varepsilon^{-N}
$$

Teorema 3.1.29 Sejam $J$ um intervalo aberto limitado de $\mathbb{R}, \quad F \in \mathcal{G}_{\tau}\left(J \times \mathbb{R}^{n} ; \mathbb{R}^{n}\right)$, $x_{0} \in \overline{\mathbb{R}}^{n}$ e $t_{0} \in J$. Se existe um representante $\left(F_{\varepsilon}\right)_{\varepsilon}$ de $F$ tal que $\left(\nabla_{x} F_{\varepsilon}\right)_{\varepsilon}$ é $L^{\infty}$ - ln -tipo em $\mathcal{E}_{\tau}\left[J \times \mathbb{R}^{n} ; \mathbb{R}^{n^{2}}\right]$, então o problema $(3.3)$ tem uma única solução $u \in \mathcal{G}_{\tau}\left(J ; \mathbb{R}^{n}\right)$. 
Demonstração: Seja $\left(x_{0_{\varepsilon}}\right)_{\varepsilon} \in\left(\mathcal{E}_{M}(\mathbb{R})\right)^{n}$ um representante de $x_{0}$.

Da Proposição 3.1.20, existe $\left(u_{\varepsilon}\right)_{\varepsilon} \in\left(\mathscr{C}^{\infty}\left(J ; \mathbb{R}^{n}\right)\right)^{\mathbf{I}}$ tal que

$$
\begin{aligned}
& \frac{d u_{\varepsilon}}{d t}(t)=F_{\varepsilon}\left(t, u_{\varepsilon}(t)\right), \quad \forall(\varepsilon, t) \in \mathbf{I} \times J, \\
& u_{\varepsilon}\left(t_{0}\right)=x_{0_{\varepsilon}}
\end{aligned}
$$

e além disso, $\left(u_{\varepsilon}\right)_{\varepsilon} \in \mathcal{E}_{\tau}\left[J ; \mathbb{R}^{n}\right]$. Assim, $u:=\left(u_{\varepsilon}\right)_{\varepsilon}+\mathcal{N}_{\tau}\left[J ; \mathbb{R}^{n}\right]$ é uma solução de (3.3).

Seja $v=\left[\left(v_{\varepsilon}\right)_{\varepsilon}\right]$ uma outra solução para (3.3). Logo existem $\left(n_{\varepsilon}\right)_{\varepsilon} \in \mathcal{N}_{\tau}\left[J ; \mathbb{R}^{n}\right]$ e $\left(m_{\varepsilon}\right)_{\varepsilon} \in(\mathcal{N}(\mathbb{R}))^{n}$, tais que

$$
\begin{aligned}
\frac{d u_{\varepsilon}}{d t}(t)-\frac{d v_{\varepsilon}}{d t}(t) & =F_{\varepsilon}\left(t, u_{\varepsilon}(t)\right)-F_{\varepsilon}\left(t, v_{\varepsilon}(t)\right)+n_{\varepsilon}(t), \quad \forall(\varepsilon, t) \in \mathbf{I} \times J ; \\
u_{\varepsilon}\left(t_{0}\right)-v_{\varepsilon}\left(t_{0}\right) & =m_{\varepsilon}, \quad \forall \varepsilon \in \mathbf{I} .
\end{aligned}
$$

Provaremos que $\left(u_{\varepsilon}-v_{\varepsilon}\right)_{\varepsilon} \in \mathcal{N}_{\tau}\left[J ; \mathbb{R}^{n}\right]$, e assim $u=v$.

Considere $T \in \mathbb{N}$ tal que $J \subset[-T, T]$.

De $\left(\nabla_{x} F_{\varepsilon}\right)_{\varepsilon}$ ser $L^{\infty}$ - ln-tipo em $\mathcal{E}_{\tau}\left[J \times \mathbb{R}^{n} ; \mathbb{R}^{n^{2}}\right]$, existem $c_{1}>0$ e $\eta_{1} \in \mathbf{I}$, tais que

$$
\left|\nabla_{x} F_{\varepsilon}(t, y)\right| \leq c_{1}|\ln \varepsilon|, \quad \forall(\varepsilon, t, y) \in \mathbf{I}_{\eta_{1}} \times J \times \mathbb{R}^{n}
$$

De $\left(n_{\varepsilon}\right)_{\varepsilon} \in \mathcal{N}_{\tau}\left[J ; \mathbb{R}^{n}\right]$, existe $N_{1} \in \mathbb{N}$ tal que, para todo $q \in \mathbb{N}$, existem $c_{q}>c_{1}$ e $\eta_{q} \in \mathbf{I}$ com $\eta_{q}<\eta_{1}$ satisfazendo

$$
\left|n_{\varepsilon}(t)\right| \leq c_{q}(1+|t|)^{N_{1}} \varepsilon^{q}, \quad \forall(\varepsilon, t) \in \mathbf{I}_{\eta_{q}} \times J
$$

Sejam $N=1$ e $r \in \mathbb{N}$. Considere $q=r+2 T n c_{1}$ e sejam $c_{2}:=c_{q}$ e $\eta_{2}:=\eta_{q}$ como em $(3.52)$

De $\left(m_{\varepsilon}\right)_{\varepsilon} \in(\mathcal{N}(\mathbb{R}))^{n}$, existem $c_{3}>c_{2}$ e $\eta_{3} \in \mathbf{I}$ com $\eta_{3}<\eta_{2}$ tais que

$$
\left|m_{\varepsilon}\right| \leq c_{3} \varepsilon^{q}, \quad \forall \varepsilon \in \mathbf{I}_{\eta_{3}}
$$

Notemos que

$$
\int_{t_{0}}^{t}\left(F_{\varepsilon}\left(s, u_{\varepsilon}(s)\right)-F_{\varepsilon}\left(s, v_{\varepsilon}(s)\right)+n_{\varepsilon}(s)\right) d s=\left(u_{\varepsilon}(t)-v_{\varepsilon}(t)\right)-\left(u_{\varepsilon}\left(t_{0}\right)-v_{\varepsilon}\left(t_{0}\right)\right),
$$

e assim

$$
u_{\varepsilon}(t)-v_{\varepsilon}(t)=m_{\varepsilon}+\int_{t_{0}}^{t}\left(F_{\varepsilon}\left(s, u_{\varepsilon}(s)\right)-F_{\varepsilon}\left(s, v_{\varepsilon}(s)\right)+n_{\varepsilon}(s)\right) d s
$$


para todo $(\varepsilon, t) \in \mathbf{I} \times J$.

Suponhamos $\left(F_{\varepsilon}\right)_{\varepsilon}:=\left(\left(F_{1_{\varepsilon}}, \cdots, F_{n_{\varepsilon}}\right)\right)_{\varepsilon}, \quad\left(u_{\varepsilon}\right)_{\varepsilon}:=\left(\left(u_{1_{\varepsilon}}, \cdots, u_{n_{\varepsilon}}\right)\right)_{\varepsilon}$, $\left(n_{\varepsilon}\right)_{\varepsilon}:=\left(\left(n_{1_{\varepsilon}}, \cdots, n_{n_{\varepsilon}}\right)\right)_{\varepsilon} \quad$ e $\quad\left(m_{\varepsilon}\right)_{\varepsilon}:=\left(\left(m_{1_{\varepsilon}}, \cdots, m_{n_{\varepsilon}}\right)\right)_{\varepsilon}$.

Fixemos $1 \leq i \leq n$.

Para facilitar a escrita considere $\left(G_{\varepsilon}\right)_{\varepsilon}:=\left(F_{i_{\varepsilon}}\right)_{\varepsilon}$. Então, de (3.51), (3.52), (3.53) e (3.54), temos que

$$
\begin{gathered}
\left|\nabla_{x} G_{\varepsilon}(t, y)\right| \leq c_{1}|\ln \varepsilon|, \quad \forall(\varepsilon, t, y) \in \mathbf{I}_{\eta_{3}} \times J \times \mathbb{R}^{n} ; \\
\left|m_{i_{\varepsilon}}\right| \leq c_{3} \varepsilon^{q}, \quad \forall \varepsilon \in \mathbf{I}_{\eta_{3}} ; \\
\left|n_{i_{\varepsilon}}(t)\right| \leq c_{2}(1+|t|)^{N_{1}} \varepsilon^{q}, \quad \forall(\varepsilon, t) \in \mathbf{I}_{\eta_{3}} \times J ; \\
u_{i_{\varepsilon}}(t)-v_{i_{\varepsilon}}(t)=m_{i_{\varepsilon}}+\int_{t_{0}}^{t}\left(G_{\varepsilon}\left(s, u_{\varepsilon}(s)\right)-G_{\varepsilon}\left(s, v_{\varepsilon}(s)\right)+n_{i_{\varepsilon}}(s)\right) d s, \forall(\varepsilon, t) \in \mathbf{I}_{\eta_{3}} \times J .
\end{gathered}
$$

Fixemos $(\varepsilon, t) \in \mathbf{I}_{\eta_{3}} \times J$.

De (3.56), (3.57) e (3.58), temos

$$
\begin{aligned}
\left|u_{i_{\varepsilon}}(t)-v_{i_{\varepsilon}}(t)\right| & =\left|m_{i_{\varepsilon}}+\int_{t_{0}}^{t}\left(G_{\varepsilon}\left(s, u_{\varepsilon}(s)\right)-G_{\varepsilon}\left(s, v_{\varepsilon}(s)\right)+n_{i_{\varepsilon}}(s)\right) d s\right| \\
& \leq\left|m_{i_{\varepsilon}}\right|+\left|\int_{t_{0}}^{t}\right| G_{\varepsilon}\left(s, u_{\varepsilon}(s)\right)-G_{\varepsilon}\left(s, v_{\varepsilon}(s)\right)|d s|+\left|\int_{t_{0}}^{t}\right| n_{i_{\varepsilon}}(s)|d s| \\
& \leq c_{3} \varepsilon^{q}+\left|\int_{t_{0}}^{t} c_{2}(1+|s|)^{N_{1}} \varepsilon^{q} d s\right|+\left|\int_{t_{0}}^{t}\right| G_{\varepsilon}\left(s, u_{\varepsilon}(s)\right)-G_{\varepsilon}\left(s, v_{\varepsilon}(s)\right)|d s| \\
& \leq c_{3} \varepsilon^{q}+c_{2}(1+T)^{N_{1}}\left(|t|+\left|t_{0}\right|\right) \varepsilon^{q}+\left|\int_{t_{0}}^{t}\right| G_{\varepsilon}\left(s, u_{\varepsilon}(s)\right)-G_{\varepsilon}\left(s, v_{\varepsilon}(s)\right)|d s| \\
& \leq c_{3}\left(1+(1+T)^{N_{1}} 2 T\right) \varepsilon^{q}+\left|\int_{t_{0}}^{t}\right| G_{\varepsilon}\left(s, u_{\varepsilon}(s)\right)-G_{\varepsilon}\left(s, v_{\varepsilon}(s)\right)|d s| .
\end{aligned}
$$

Usando o Teorema do Valor Médio na segunda parcela da última desigualdade acima temos

$$
\left|u_{i_{\varepsilon}}(t)-v_{i_{\varepsilon}}(t)\right| \leq c_{2}\left(1+(1+T)^{N_{1}} 2 T\right) \varepsilon^{q}+\left|\int_{t_{0}}^{t}\right| u_{\varepsilon}(s)-v_{\varepsilon}(s)|| \nabla_{x} G_{\varepsilon}\left(s, w_{\varepsilon}(s)\right)|d s|,
$$

onde $w_{\varepsilon}(s):=u_{\varepsilon}(s)+\sigma_{s}\left(u_{\varepsilon}(s)-v_{\varepsilon}(s)\right)$ para algum $\sigma_{s} \in[0,1]$. Assim, de (3.55), segue que 


$$
\left|u_{i_{\varepsilon}}(t)-v_{i_{\varepsilon}}(t)\right| \leq c_{3} \varepsilon^{q}+\left|\int_{t_{0}}^{t}\right| u_{\varepsilon}(s)-v_{\varepsilon}(s)\left|c_{1}\right| \ln \varepsilon|d s|,
$$

onde $c_{4}:=c_{3}\left(1+(1+T)^{N_{1}} 2 T\right)$. Logo

$$
\left|u_{\varepsilon}(t)-v_{\varepsilon}(t)\right| \leq n c_{3} \varepsilon^{q}+\left|\int_{t_{0}}^{t} n c_{1}\right| \ln \varepsilon|| u_{\varepsilon}(s)-v_{\varepsilon}(s)|d s|, \quad \forall(\varepsilon, t) \in \mathbf{I}_{\eta_{2}} \times J .
$$

Dessa forma, pelo Lema 3.1.16, tem-se, para todo $(\varepsilon, t) \in \mathbf{I}_{\eta_{3}} \times J$, que

$$
\begin{aligned}
\left|u_{\varepsilon}(t)-v_{\varepsilon}(t)\right| & \leq n c_{3} \varepsilon^{q} \exp \left(\left|\int_{t_{0}}^{t} n c_{1}\right| \ln \varepsilon|d s|\right) \\
& \leq n c_{3} \varepsilon^{q} \exp \left(-2 T n c_{1} \ln \varepsilon\right) \\
& \leq n c_{3} \varepsilon^{q} \exp \left(\ln \varepsilon^{-2 T n c_{1}}\right) \\
& \leq n c_{3} \varepsilon^{q-2 T n c_{1}} \leq c(1+|t|) \varepsilon^{r}
\end{aligned}
$$

onde $c:=n c_{3}$. Portanto $\left(u_{\varepsilon}-v_{\varepsilon}\right)$ satisfaz as hipóteses do Teorema de Nulidade para funções em $\mathcal{E}_{\tau}\left[J ; \mathbb{R}^{n}\right]$ (Teorema 1.3.7). Desse fato concluímos que $\left(u_{\varepsilon}-v_{\varepsilon}\right)_{\varepsilon} \in \mathcal{N}_{\tau}\left[J ; \mathbb{R}^{n}\right]$, ou seja, o problema (3.3) admite uma única solução em $\mathcal{G}_{\tau}\left(J ; \mathbb{R}^{n}\right)$ se $\left(F_{\varepsilon}\right)_{\varepsilon} \in \mathcal{E}_{\tau}\left[J \times \mathbb{R}^{n} ; \mathbb{R}^{n}\right]$ é tal que $\left(\nabla_{x} F_{\varepsilon}\right)_{\varepsilon}$ é $L^{\infty}$-ln-tipo em $\mathcal{E}_{\tau}\left[J \times \mathbb{R}^{n} ; \mathbb{R}^{n^{2}}\right]$.

Finalizamos esta seção com a seguinte observação.

Observação 3.1.30 Sejam $J$ um intervalo aberto de $\mathbb{R}, t_{0} \in J, \quad F \in \mathcal{G}_{\tau}\left(J \times \mathbb{R}^{s n} ; \mathbb{R}^{n}\right)$ (respectivamente $\left.\widetilde{\mathcal{G}}_{\tau}\left(J \times \mathbb{R}^{s n} ; \mathbb{R}^{n}\right)\right),\left(F_{\varepsilon}\right)_{\varepsilon}$ um representante de $F,\left(G_{\varepsilon}\right)_{\varepsilon}$ definida por

$$
G_{\varepsilon}\left(t, y_{1}, y_{2}, \cdots, y_{s n}\right)=\left(y_{n+1}, \cdots, y_{s n}, F_{\varepsilon}\left(t, y_{1}, y_{2}, \cdots, y_{s n}\right)\right)
$$

e $G:=\left[\left(G_{\varepsilon}\right)_{\varepsilon}\right] \in \mathcal{G}_{\tau}\left(J \times \mathbb{R}^{s n} ; \mathbb{R}^{s n}\right) \quad\left(\right.$ resp. $\left.\quad \widetilde{\mathcal{G}}_{\tau}\left(J \times \mathbb{R}^{s n} ; \mathbb{R}^{s n}\right)\right) . \quad$ Considere os seguintes problemas:

(I) determinar $v \in \mathcal{G}\left(J ; \mathbb{R}^{n}\right)$ tal que

$$
\left(v\left(t_{0}\right), \frac{d v}{d t}\left(t_{0}\right), \cdots, \frac{d^{s-1} v}{d t^{s-1}}\left(t_{0}\right)\right)=\left(x_{0}^{1}, \cdots, x_{0}^{s n}\right) \in \overline{\mathbb{R}}^{s n} \quad e \frac{d^{s} v}{d t^{s}}=F \circ\left(i d, v, \frac{d v}{d t}, \cdots, \frac{d^{s-1} v}{d t^{s-1}}\right)
$$


(II) determinar $u \in \mathcal{G}\left(J ; \mathbb{R}^{s n}\right)$ tal que

$$
u\left(t_{0}\right)=\left(x_{0}^{1}, \cdots, x_{0}^{s n}\right) \in \overline{\mathbb{R}}^{s n} \quad e \frac{d u}{d t}=G \circ(i d, u)
$$

Então, se v é solução de $(I)$ tem-se que $u=\left(v, \frac{d v}{d t}, \cdots, \frac{d^{s-1} v}{d t^{s-1}}\right)$ é solução de $(I I)$. Por outro lado, se $u=\left(u_{1}, \cdots, u_{n}, \cdots, u_{s n}\right)$ é solução de $(I I)$, então $v=\left(u_{1}, \cdots, u_{n}\right)$ é solução de $(I)$.

Da Observação 3.1.30 e com os resultados desta seção obtem-se resultados para Equações Diferenciais Ordinárias de ordem n no contexto das funções generalizadas temperadas (ou no contexto das funções generalizadas temperadas na segunda variável). Uma aplicação desse fato será feita na próxima seção.

\subsection{As equações de Euler-Lagrange - um exemplo}

Iniciaremos esta seção com um breve comentário sobre as equações de Euler-Lagrange. Antes porém, definimos, para $k \in \mathbb{N}^{*}$ e $a, b \in \mathbb{R}$ com $a<b$, os seguintes conjuntos:

- $\mathscr{C}^{k}([a, b] ; \mathbb{R}):=\left\{\varphi \in \mathbb{R}^{[a, b]} \mid \exists s>0\right.$ e $\exists \psi \in \mathscr{C}^{k}(] a-s, b+s[; \mathbb{R})$

$$
\text { tais que } \left.\varphi=\left.\psi\right|_{[a, b]}\right\} \text {; }
$$

- $\mathscr{C}^{k}\left([a, b] \times \mathbb{R}^{n} \times \mathbb{R}^{n} ; \mathbb{R}\right):=\left\{\varphi \in \mathbb{R}^{[a, b] \times R^{n} \times R^{n}} \mid \exists s>0 \mathrm{e}\right.$

$$
\left.\exists \psi \in \mathscr{C}^{k}(] a-s, b+s\left[\times \mathbb{R}^{n} \times \mathbb{R}^{n} ; \mathbb{R}\right) \text { tais que } \varphi=\left.\psi\right|_{[a, b] \times R^{n} \times R^{n}}\right\} \text {. }
$$

Considere em $\mathscr{C}^{1}\left([a, b] ; \mathbb{R}^{n}\right)$ a norma definida por

$$
\|u\|=\max \left\{|u(t)|+\left|\frac{d u}{d t}(t)\right| \mid t \in[a, b]\right\} .
$$


Sejam $A, B \in \mathbb{R}^{n}, \quad \mathscr{D}=\left\{w \in \mathscr{C}^{1}\left([a, b] ; \mathbb{R}^{n}\right) \mid w(a)=A\right.$ e $\left.w(b)=B\right\}$, $L=L(t, x, y) \in \mathscr{C}^{1}\left([a, b] \times \mathbb{R}^{n} \times \mathbb{R}^{n} ; \mathbb{R}\right)$ e $\mathscr{I}[\bullet]$ a função definida por

$$
\mathscr{I}[w]=\int_{a}^{b} L\left(t, w(t), \frac{d w}{d t}(t)\right) d t, \quad \forall w \in \mathscr{D} .
$$

Prova-se que, se $u$ é um ponto de mínimo local (ou de máximo local) de $\mathscr{I}[\bullet]$, então $u$ satisfaz as equações

$$
\left.\frac{d}{d t}\left(\frac{\partial L}{\partial y}\left(t, u(t), \frac{d u}{d t}(t)\right)\right)=\frac{\partial L}{\partial x}\left(t, u(t), \frac{d u}{d t}(t)\right), \quad \forall t \in\right] a, b[
$$

onde $\quad \frac{\partial L}{\partial y}:=\left(\frac{\partial L}{\partial y_{1}}, \cdots, \frac{\partial L}{\partial y_{n}}\right) \quad$ e $\quad \frac{\partial L}{\partial x}:=\left(\frac{\partial L}{\partial x_{1}}, \cdots, \frac{\partial L}{\partial x_{n}}\right), \quad$ sendo que $(x, y):=\left(x_{1}, \cdots, x_{n}, y_{1}, \cdots, y_{n}\right)$ denota um ponto arbitrário de $\mathbb{R}^{n} \times \mathbb{R}^{n}$. Uma prova desse fato pode ser encontrada, por exemplo, em [Tro].

As equações obtidas em (3.59) são chamadas de equações de Euler-Lagrange associadas à função $\mathscr{I}[\bullet]$.

Note que, se $V \in \mathscr{C}^{\infty}\left(\mathbb{R}^{n} ; \mathbb{R}\right)$ e $L=L(t, x, y)$ é a função definida por

$$
L(t, x, y):=\frac{|y|^{2}}{2}-V(x), \quad \forall(t, x, y) \in \mathbb{R} \times \mathbb{R}^{n} \times \mathbb{R}^{n}
$$

então $\frac{\partial L}{\partial y}(t, x, y)=y \quad$ e $\quad \frac{\partial L}{\partial x}(t, x, y)=-\nabla V(x)$, onde $\nabla V$ denota o gradiente de $V$. Assim, (3.59) é da forma

$$
\frac{d}{d t}\left(\frac{d u}{d t}(t)\right)=-\nabla V(u(t))
$$

ou seja

$$
\frac{d^{2} u}{d t^{2}}(t)+\nabla V(u(t))=0
$$

Nesta seção iremos estudar (3.60) no contexto das funções generalizadas temperadas de Colombeau. Mais precisamente, 
Dados $t_{0} \in \mathbb{R}, u_{0}, v_{0} \in \overline{\mathbb{R}}^{n}$ e $V \in \mathcal{G}_{\tau}\left(\mathbb{R}^{n} ; \mathbb{R}\right)$, iremos determinar algumas condições de modo a garantir a existência de pelo menos uma (ou somente uma) $u \in \mathcal{G}\left(\mathbb{R}^{n} ; \mathbb{R}^{n}\right)$ tal que

$$
\begin{aligned}
& \frac{d^{2} u}{d t^{2}}+\nabla V \circ u=0 \\
& \left(u\left(t_{0}\right), \frac{d u}{d t}\left(t_{0}\right)\right)=\left(u_{0}, v_{0}\right)
\end{aligned}
$$

Para essa finalidade, introduziremos a seguinte notação:

Se $g \in \mathscr{C}^{\infty}\left(\mathbb{R}^{n} ; \mathbb{R}\right)$, denotamos por $\nabla^{2} g$ o elemento de $\mathscr{C}^{\infty}\left(\mathbb{R}^{n} ; \mathbb{R}^{n^{2}}\right)$ definido por $\nabla^{2} g:=\left(\frac{\partial^{2} g}{\partial x_{1} \partial x_{1}}, \cdots, \frac{\partial^{2} g}{\partial x_{n} \partial x_{1}}, \frac{\partial^{2} g}{\partial x_{1} \partial x_{2}}, \cdots, \frac{\partial^{2} g}{\partial x_{n} \partial x_{2}}, \cdots, \frac{\partial^{2} g}{\partial x_{1} \partial x_{n}}, \cdots, \frac{\partial^{2} g}{\partial x_{n} \partial x_{n}}\right)$

onde $x:=\left(x_{1}, \cdots, x_{n}\right)$ denota um ponto arbitrário de $\mathbb{R}^{n}$.

Note que, se $\left(h_{\varepsilon}\right)_{\varepsilon}$ e $\left(g_{\varepsilon}\right)_{\varepsilon}$ são representantes de $h \in \mathcal{G}_{\tau}\left(\mathbb{R}^{n} ; \mathbb{R}\right)$, então $\left(\nabla^{2} h_{\varepsilon}-\nabla^{2} g_{\varepsilon}\right)_{\varepsilon} \in \mathcal{N}_{\tau}\left[\mathbb{R}^{n} ; \mathbb{R}^{n^{2}}\right]$. Logo, faz sentido a seguinte notação.

Se $h \in \mathcal{G}_{\tau}\left(\mathbb{R}^{n} ; \mathbb{R}\right)$, denotaremos por $\nabla^{2} h$ a classe de $\left(\nabla^{2} h_{\varepsilon}\right)_{\varepsilon}$ em $\mathcal{G}_{\tau}\left(\mathbb{R}^{n} ; \mathbb{R}^{n^{2}}\right)$, onde $\left(h_{\varepsilon}\right)_{\varepsilon} \in \mathcal{E}_{\tau}\left[\mathbb{R}^{n} ; \mathbb{R}\right]$ é um representante qualquer de $h$.

Da Observação 3.1.30 e do Teorema 3.1.21 (2.), temos o seguinte teorema de existência e unicidade para o problema (3.61).

Teorema 3.2.1 Seja $V \in \mathcal{G}_{\tau}\left(\mathbb{R}^{n} ; \mathbb{R}\right)$. Se existe um representante $\left(V_{\varepsilon}\right)_{\varepsilon}$ de $V$ tal que $\left(\nabla^{2} V_{\varepsilon}\right)_{\varepsilon}$ é $L^{\infty}$-ln -tipo em $\mathcal{E}_{\tau}\left[\mathbb{R}^{n} ; \mathbb{R}^{n^{2}}\right]$, então o problema (3.61) tem uma única solução em $\mathcal{G}\left(\mathbb{R} ; \mathbb{R}^{n}\right)$.

Demonstração: Sejam $u_{0}, v_{0} \in \overline{\mathbb{R}}^{n}$ e $\left(F_{\varepsilon}\right)_{\varepsilon} \in \mathcal{E}_{\tau}\left[\mathbb{R} \times \mathbb{R}^{2 n} ; \mathbb{R}^{2 n}\right]$ definida por

$$
F_{\varepsilon}\left(t, x_{1}, \cdots, x_{n}, y_{1}, \cdots, y_{n}\right)=\left(y_{1}, \cdots, y_{n},-\nabla V_{\varepsilon}\left(x_{1} \cdots, x_{n}\right)\right)
$$


onde $\left(V_{\varepsilon}\right)_{\varepsilon}$ é um representante de $V$ tal que $\left(\nabla^{2} V_{\varepsilon}\right)_{\varepsilon}$ é $L^{\infty}$-ln-tipo em $\mathcal{E}_{\tau}\left[\mathbb{R}^{n} ; \mathbb{R}^{n^{2}}\right]$ e $(x, y):=\left(x_{1}, \cdots, x_{n}, y_{1}, \cdots, y_{n}\right)$ denota um ponto arbitrário de $\mathbb{R}^{n} \times \mathbb{R}^{n}$.

Como $\left(\nabla^{2} V_{\varepsilon}\right)_{\varepsilon}$ é $L^{\infty}$-ln-tipo em $\mathcal{E}_{\tau}\left[\mathbb{R}^{n} ; \mathbb{R}^{n^{2}}\right]$, é claro que $\left(\nabla_{(x, y)} F_{\varepsilon}\right)_{\varepsilon}$ é $L^{\infty}$ - ln-tipo em $\mathcal{E}_{\tau}\left[\mathbb{R} \times \mathbb{R}^{2 n} ; \mathbb{R}^{4 n^{2}}\right]$. Assim, se $F=\left[\left(F_{\varepsilon}\right)_{\varepsilon}\right] \in \mathcal{G}_{\tau}\left(\mathbb{R} \times \mathbb{R}^{2 n} ; \mathbb{R}^{2 n}\right)$ temos, pelo Teorema 3.1 .21 (2.), que existe uma única $w:=\left(w_{1}, \cdots, w_{2 n}\right) \in \mathcal{G}\left(\mathbb{R} ; \mathbb{R}^{2 n}\right)$ tal que

$$
\frac{d w}{d t}=F \circ(i d, w) \quad \text { e } \quad w\left(t_{0}\right)=\left(u_{0}, v_{0}\right) \in \overline{\mathbb{R}}^{2 n} .
$$

Logo, pela Observação 3.1.30, $u:=\left(w_{1}, \cdots, w_{n}\right) \in \mathcal{G}\left(\mathbb{R} ; \mathbb{R}^{n}\right)$ é solução de $(3.61)$.

Seja $v=\left(v_{1}, \cdots, v_{n}\right) \in \mathcal{G}\left(\mathbb{R} ; \mathbb{R}^{n}\right)$ uma outra solução de (3.61). Novamente, pela Observação 3.1.30, temos que $X=\left(v, \frac{d v}{d t}\right) \in \mathcal{G}\left(\mathbb{R} ; \mathbb{R}^{2 n}\right)$ é uma solução de (3.62). Como existe uma única solução de $(3.62)$ em $\mathcal{G}\left(\mathbb{R} ; \mathbb{R}^{2 n}\right)$, temos que $X=w$, e assim $v=\left(v_{1}, \cdots, v_{n}\right)=\left(w_{1} \cdots, w_{n}\right)=u \operatorname{em~} \mathcal{G}\left(\mathbb{R} ; \mathbb{R}^{n}\right)$.

Em [GKOS], além do resultado apresentado no Teorema 3.2.1, existem outros resultados de existência de solução para o problema (3.61) nos quais, as condições são sobre $V$ e não sobre $\nabla^{2} V$. Apresentaremos, a seguir, esses resultados. Como foi feito na seção anterior, iniciaremos estudando o problema (3.61) a nível de representantes.

Proposição 3.2.2 Sejam $\left(V_{\varepsilon}\right)_{\varepsilon} \in \mathcal{E}_{\tau}\left[\mathbb{R}^{n} ; \mathbb{R}\right]$ e $\left(u_{\varepsilon}\right)_{\varepsilon} \in\left(\mathscr{C}^{\infty}\left(\mathbb{R} ; \mathbb{R}^{n}\right)\right)^{\mathbf{I}}$ tais que

(i) $\frac{d^{2} u_{\varepsilon}}{d t^{2}}(t)+\nabla V_{\varepsilon}\left(u_{\varepsilon}(t)\right)=0, \quad \forall(\varepsilon, t) \in \mathbf{I} \times \mathbb{R} ;$

(ii) $\exists N_{1} \in \mathbb{N}, \exists c_{1}>0$ e $\exists \eta_{1} \in \mathbf{I}$ tais que

$$
\begin{aligned}
& \text { (a) }\left|u_{\varepsilon}(t)\right| \leq c_{1}(1+|t|)^{N_{1}} \varepsilon^{-N_{1}}, \quad \forall(\varepsilon, t) \in \mathbf{I}_{\eta_{1}} \times \mathbb{R}, \\
& \text { (b) }\left|\frac{d u_{\varepsilon}}{d t}(t)\right| \leq\left(c_{1} \varepsilon^{-N_{1}}+2\left|V_{\varepsilon}\left(u_{\varepsilon}(t)\right)\right|\right)^{1 / 2}, \quad \forall(\varepsilon, t) \in \mathbf{I}_{\eta_{1}} \times \mathbb{R} .
\end{aligned}
$$

\section{Então}

1. $\left(u_{\varepsilon}\right)_{\varepsilon} \in \mathcal{E}_{\tau}\left[\mathbb{R} ; \mathbb{R}^{n}\right]$; 
2. $\left(u_{\varepsilon}\right)_{\varepsilon} \in \mathcal{E}_{M}\left[\mathbb{R} ; \mathbb{R}^{n}\right]$.

Demonstração: Como 2. decorre diretamente de 1. e da Proposição 1.3.12 (1.), basta provarmos que 1. é verdadeira. Para tal, mostraremos, pelo Princípio de Indução Finita, que para todo $p \in \mathbb{N}$, existem $N \in \mathbb{N}, c>0$ e $\eta \in \mathbf{I}$ tais que

$$
\left|\frac{d^{p} u_{\varepsilon}}{d t^{p}}(t)\right| \leq c(1+|t|)^{N} \varepsilon^{-N}, \quad \forall(\varepsilon, t) \in \mathbf{I}_{\eta} \times \mathbb{R} .
$$

Seja $p \in \mathbb{N}$.

Sejam $N_{1} \in \mathbb{N}, c_{1}>0$ e $\eta_{1} \in \mathbf{I}$ como em $(i i)$. De $\left(V_{\varepsilon}\right)_{\varepsilon} \in \mathcal{E}_{\tau}\left[\mathbb{R}^{n} ; \mathbb{R}\right]$, existem $N_{2} \geq N_{1}$, $c_{2} \geq \max \left\{1, c_{1}\right\}$ e $\eta_{2} \in \mathbf{I}$ com $\eta_{2} \leq \eta_{1}$ tais que

$$
\left|\partial^{\alpha} V_{\varepsilon}(y)\right| \leq c_{2}(1+|y|)^{N_{2}} \varepsilon^{-N_{2}}, \quad \forall(\varepsilon, y) \in \mathbf{I}_{\eta_{2}} \times \mathbb{R}^{n}, \quad \forall \alpha \in \mathbb{N}^{n} \text { com }|\alpha| \leq p .
$$

Portanto, por (ii) (a), temos, para $(\varepsilon, t) \in \mathbf{I}_{\eta_{2}} \times \mathbb{R}$ e $\alpha \in \mathbb{N}^{n}$ com $|\alpha| \leq p$ que

$$
\begin{aligned}
\left|\partial^{\alpha} V_{\varepsilon}\left(u_{\varepsilon}(t)\right)\right| & \leq c_{2}\left(1+\left|u_{\varepsilon}(t)\right|\right)^{N_{2}} \varepsilon^{-N_{2}} \\
& \leq c_{2}\left(1+c_{1}(1+|t|)^{N_{1}} \varepsilon^{-N_{1}}\right)^{N_{2}} \varepsilon^{-N_{2}} \\
& \leq c_{2}\left((1+|t|)^{N_{1}} \varepsilon^{-N_{1}}+c_{1}(1+|t|)^{N_{1}} \varepsilon^{-N_{1}}\right)^{N_{2}} \varepsilon^{-N_{2}} \\
& \leq c_{2}(1+|t|)^{N_{1} N_{2}}\left(1+c_{1}\right)^{N_{2}} \varepsilon^{-N_{1} N_{2}} \varepsilon^{-N_{2}} \\
& \leq c_{2}(1+|t|)^{N_{2}^{2}}\left(1+c_{1}\right)^{N_{2}} \varepsilon^{-\left(N_{2}^{2}+N_{2}\right)} \\
& \leq c_{2}\left(1+c_{1}\right)^{N_{2}}(1+|t|)^{N_{2}^{2}+N_{2}} \varepsilon^{-\left(N_{2}^{2}+N_{2}\right)} .
\end{aligned}
$$

Portanto, para $N_{3}:=N_{2}^{2}+N_{2}$ e $c_{3}:=c_{2}\left(1+c_{1}\right)^{N_{2}}$, temos

$$
\left|\partial^{\alpha} V_{\varepsilon}\left(u_{\varepsilon}(t)\right)\right| \leq c_{3}(1+|t|)^{N_{3}} \varepsilon^{-N_{3}}, \quad \forall(\varepsilon, t) \in \mathbf{I}_{\eta_{2}} \times \mathbb{R}, \quad \forall \alpha \in \mathbb{N}^{n}, \text { com }|\alpha| \leq p . \text { (3.64) }
$$

Note ainda que, de (3.64) e (ii) (b), temos, para $(\varepsilon, t) \in \mathbf{I}_{\eta_{2}} \times \mathbb{R}$, que

$$
\begin{aligned}
\left|\frac{d u_{\varepsilon}}{d t}(t)\right| & \leq\left(c_{1} \varepsilon^{-N_{1}}+2\left|V_{\varepsilon}\left(u_{\varepsilon}(t)\right)\right|\right)^{1 / 2} \\
& \leq\left(c_{1} \varepsilon^{-N_{1}}+2 c_{3}(1+|t|)^{N_{3}} \varepsilon^{-N_{3}}\right)^{1 / 2} \\
& \leq\left(c_{1} \varepsilon^{-N_{3}}+2 c_{3}(1+|t|)^{N_{3}} \varepsilon^{-N_{3}}\right)^{1 / 2} \\
& \leq\left(\left(c_{1}+2 c_{3}\right)(1+|t|)^{N_{3}} \varepsilon^{-N_{3}}\right)^{1 / 2} \\
& \leq\left(3 c_{3}\right)(1+|t|)^{N_{3}} \varepsilon^{-N_{3}},
\end{aligned}
$$


ou seja

$$
\left|\frac{d u_{\varepsilon}}{d t}(t)\right| \leq\left(3 c_{3}\right)(1+|t|)^{N_{3}} \varepsilon^{-N_{3}}, \quad \forall(\varepsilon, t) \in \mathbf{I}_{\eta_{2}} \times \mathbb{R} .
$$

Para $p=0$ ou $p=1$ temos, por $(i i)(a)$ e (3.65), que (3.63) é verdadeira.

Suponhamos $p \geq 2$ e que (3.63) seja verdadeira para todo $s \in \mathbb{N}$ com $s \leq p-1$. Logo, existem $N_{4} \in \mathbb{N}, c_{4}>0$ e $\eta_{3} \in \mathbf{I}$ com $\eta_{3}<\eta_{2}$ tais que

$$
\left|\frac{d^{s} u_{\varepsilon}}{d t^{s}}(t)\right| \leq c_{4}(1+|t|)^{N_{4}} \varepsilon^{-N_{4}}, \quad \forall(\varepsilon, t) \in \mathbf{I}_{\eta_{3}} \times \mathbb{R}, \forall s \in \mathbb{N} \text { com } s \leq p-1 .
$$

Admita que $\left(u_{\varepsilon}\right)_{\varepsilon}:=\left(\left(u_{1_{\varepsilon}}, \cdots, u_{n_{\varepsilon}}\right)\right)_{\varepsilon}$. Para $(\varepsilon, t) \in \mathbf{I}_{\eta_{3}} \times \mathbb{R}$ fixado, considere os conjuntos

$$
\begin{gathered}
A:=\left\{\frac{d^{s} u_{i_{\varepsilon}}}{d t^{s}}(t) \mid 0 \leq s \leq p-1 \text { e } 1 \leq i \leq n\right\} ; \\
B:=\left\{\partial^{\gamma} V_{\varepsilon}\left(u_{\varepsilon}(t)\right)|\gamma| \leq p-1\right\} .
\end{gathered}
$$

De $(i)$, temos que cada coordenada de $\frac{d^{p} u_{\varepsilon}}{d t^{p}}(t)$ é a soma e o produto de elementos de $A \cup B$. Portanto, de (3.64) e (3.66), temos que (3.63) é verdadeira para $p$, o que prova $2 .$.

A Proposição 3.2.2 nos fornece condições para que $\left(u_{\varepsilon}\right)_{\varepsilon}$, satisfazendo $(i)$, pertença a $\mathcal{E}_{\tau}\left[\mathbb{R} ; \mathbb{R}^{n}\right]$, e portanto a $\mathcal{E}_{M}\left[\mathbb{R} ; \mathbb{R}^{n}\right]$. Desse fato, é razoável nos perguntar quando $(i)$ é verdadeira. Para essa finalidade iremos recorrer ao seguinte resultado no contexto clássico:

Proposição 3.2.3 Sejam $\left(t_{0}, x_{0}, y_{0}\right) \in \mathbb{R} \times \mathbb{R}^{n} \times \mathbb{R}^{n}$ e $V \in \mathscr{C}^{\infty}\left(\mathbb{R}^{n} ; \mathbb{R}\right)$ tal que $V \geq 0$ ou $V$ é limitada em $\mathbb{R}^{n}$. Então existe uma única $u \in \mathscr{C}^{\infty}\left(\mathbb{R} ; \mathbb{R}^{n}\right)$ tal que

$$
\begin{aligned}
& \frac{d^{2} u}{d t^{2}}(t)+\nabla V(u(t))=0, \quad \forall t \in \mathbb{R} \\
& \left(u\left(t_{0}\right), \frac{d u}{d t}\left(t_{0}\right)\right)=\left(x_{0}, y_{0}\right)
\end{aligned}
$$


Demonstração: Da Observação 3.1.30 temos que o problema (3.67) é equivalente a encon$\operatorname{trar} w \in \mathscr{C}^{\infty}\left(\mathbb{R} ; \mathbb{R}^{n}\right)$ tal que

$$
\begin{aligned}
& \frac{d w}{d t}+G(t, w(t))=0, \quad \forall t \in \mathbb{R} \\
& w\left(t_{0}\right)=\left(x_{0}, y_{0}\right)
\end{aligned}
$$

onde $G\left(t, x_{1}, \cdots, x_{n}, y_{1}, \cdots, y_{n}\right)=\left(y_{1} \cdots, y_{n},-\nabla V\left(x_{1}, \cdots, x_{n}\right)\right)$ e $\left(t, x_{1}, \cdots, x_{n}, y_{1}, \cdots, y_{n}\right)$ denota um ponto arbitrário de $\mathbb{R} \times \mathbb{R}^{n} \times \mathbb{R}^{n}$.

Como $G \in \mathscr{C}^{\infty}\left(\mathbb{R} \times \mathbb{R}^{2 n} ; \mathbb{R}^{2 n}\right)$ temos que (3.68) admite solução maximal $w=\left(w_{1}, \cdots, w_{n}, w_{n+1}, \cdots, w_{2 n}\right)$, cujo domínio é um intervalo aberto $J$ de $\mathbb{R}$, e $w \in \mathscr{C}^{\infty}\left(J ; \mathbb{R}^{2 n}\right)$ ([Chi], Theorem 1.184, utilizando que $\left.G\right|_{R \times \Omega}$ é Lipschitz com respeito à segunda variável, onde $\Omega$ é um aberto limitado de $\mathbb{R}^{2 n} \operatorname{com}\left(x_{0}, y_{0}\right) \in \Omega$ ). Logo, se $J$ for $\mathbb{R}$ teremos que $u:=\left(w_{1}, \cdots, w_{n}\right)$ será uma solução de (3.67).

Provaremos que $J=\mathbb{R}$.

Note que, como $\frac{d^{2} u}{d t^{2}}(t)=-\nabla V(u(t))$, para todo $t \in J$, tem-se que

$$
\left\langle\frac{d^{2} u}{d t^{2}}(t), \frac{d u}{d t}(t)\right\rangle=\left\langle-\nabla V(u(t)), \frac{d u}{d t}(t)\right\rangle, \quad \forall t \in J
$$

e assim

$$
\frac{d}{d t}\left(\frac{1}{2}\left|\frac{d u}{d t}(t)\right|^{2}+V(u(t))\right)=0, \quad \forall t \in J
$$

Portanto, existe $c \in \mathbb{R}$ tal que

$$
\frac{1}{2}\left|\frac{d u}{d t}(t)\right|^{2}+V(u(t))=c, \quad \forall t \in J .
$$

Como $\left(u\left(t_{0}\right), \frac{d u}{d t}\left(t_{0}\right)\right)=\left(x_{0}, y_{0}\right)$ temos que

$$
\frac{1}{2}\left|\frac{d u}{d t}(t)\right|^{2}=\frac{1}{2}\left|y_{0}\right|^{2}+V\left(x_{0}\right)-V(u(t)), \quad \forall t \in J .
$$

De (3.69) e das hipóteses sobre $V$ tem-se que $\left|\frac{d u}{d t}\right|$ é uma função limitada em $J$, ou seja, existe $M>0$ tal que

$$
\left|\frac{d u}{d t}(t)\right| \leq M, \quad \forall t \in J
$$


Por outro lado, observe que

$$
u(t)=u\left(t_{0}\right)+\int_{t_{0}}^{t} \frac{d u}{d t}(s) d s, \quad \forall t \in J
$$

e assim

$$
|u(t)| \leq\left|x_{0}\right|+\left|\int_{t_{0}}^{t}\right| \frac{d u}{d t}(s)|d s| \leq\left|x_{0}\right|+M\left|t-t_{0}\right|, \quad \forall t \in J .
$$

Se $J \cap\left[t_{0},+\infty\left[=\left[t_{0}, b[\right.\right.\right.$ para algum $b \in \mathbb{R}$, temos, por (3.70), que $u$ é limitada em $\left[t_{0}, b[\right.$.

Como $u=\left(w_{1} \cdots, w_{n}\right)$ e $\frac{d u}{d t}=\left(\frac{d w_{1}}{d t}, \cdots, \frac{d w_{n}}{d t}\right)=\left(w_{n+1}, \cdots, w_{2 n}\right)$ e ambas são limitadas em $\left[t_{0}, b\left[\right.\right.$ concluímos que $w$ é limitada em $\left[t_{0}, b[\right.$, o que é um absurdo, pois, pelo teorema 1.186 de [Chi], sabe-se que, para todo $K \subset \subset \mathbb{R}^{2 n}$, existe $t_{K}<b$ tal que $w\left(t_{K}\right) \notin K$. Logo, $\left[t_{0},+\infty[\subset J\right.$. De modo análogo tem-se que $\left.]-\infty, t_{0}\right] \subset J$. Portanto $\left.J=\right]-\infty,+\infty[$.

A unicidade da solução de (3.67) decorre da unicidade da solução de (3.68) e da Observação 3.1.30.

O resultado clássico que foi provado na Proposição 3.2.3 nos permite fornecer um teorema de existência para o problema (3.61) (Teorema 3.2.5). Para facilitar a sua prova apresentamos a seguinte proposição.

Proposição 3.2.4 Sejam $t_{0} \in \mathbb{R}, \quad\left(u_{0_{\varepsilon}}\right)_{\varepsilon},\left(v_{0_{\varepsilon}}\right)_{\varepsilon} \in\left(\mathcal{E}_{M}(\mathbb{R})\right)^{n} \quad e \quad\left(V_{\varepsilon}\right)_{\varepsilon} \in \mathcal{E}_{\tau}\left[\mathbb{R}^{n} ; \mathbb{R}\right]$ tal que $\left(V_{\varepsilon}\right)_{\varepsilon} \quad$ é $L^{\infty}$-tipo em $\mathcal{E}_{\tau}\left[\mathbb{R}^{n} ; \mathbb{R}\right]$ ou $V_{\varepsilon} \geq 0$, para todo $\varepsilon \in \mathbf{I}$. Então existe $\left(u_{\varepsilon}\right)_{\varepsilon} \in\left(\mathscr{C}^{\infty}\left(\mathbb{R} ; \mathbb{R}^{n}\right)\right)^{\mathbf{I}}$ tal que

1. $\forall(\varepsilon, t) \in \mathbf{I} \times \mathbb{R}$, tem-se

$$
\begin{aligned}
& \frac{d^{2} u_{\varepsilon}}{d t^{2}}(t)+\nabla V_{\varepsilon}\left(u_{\varepsilon}(t)\right)=0 \\
& \left(u_{\varepsilon}\left(t_{0}\right), \frac{d u_{\varepsilon}}{d t}\left(t_{0}\right)\right)=\left(u_{0_{\varepsilon}}, v_{0_{\varepsilon}}\right)
\end{aligned}
$$

2. $\exists N_{1} \in \mathbb{N}, \exists c_{1}>0$ e $\exists \eta_{1} \in \mathbf{I}$ tais que

$$
\left|\frac{d u_{\varepsilon}}{d t}(t)\right| \leq\left(c_{1} \varepsilon^{-N_{1}}+2\left|V_{\varepsilon}\left(u_{\varepsilon}(t)\right)\right|\right)^{1 / 2}, \quad \forall(\varepsilon, t) \in \mathbf{I}_{\eta_{1}} \times \mathbb{R} ;
$$


3. $\exists N_{2} \in \mathbb{N}, \exists c_{2}>0$ e $\exists \eta_{2} \in \mathbf{I}$ tais que

$$
\left|u_{\varepsilon}(t)\right| \leq c_{2}(1+|t|)^{N_{2}} \varepsilon^{-N_{2}}, \quad \forall(\varepsilon, t) \in \mathbf{I}_{\eta_{2}} \times \mathbb{R}
$$

Demonstração: Da Proposição 3.2 .3 e das hipóteses sobre $\left(V_{\varepsilon}\right)_{\varepsilon}$, é claro que existe $\left(u_{\varepsilon}\right)_{\varepsilon} \in\left(\mathscr{C}^{\infty}\left(\mathbb{R} ; \mathbb{R}^{n}\right)\right)^{\mathbf{I}}$ satisfazendo 1 .. Antes de provarmos que $\left(u_{\varepsilon}\right)_{\varepsilon}$ também satisfaz as outras afirmações, notemos que, para $(\varepsilon, t) \in \mathbf{I} \times \mathbb{R}$, tem-se

$$
\begin{aligned}
\frac{d}{d t}\left(\frac{1}{2}\left|\frac{d u_{\varepsilon}}{d t}(t)\right|^{2}+V_{\varepsilon}\left(u_{\varepsilon}(t)\right)\right) & =\left\langle\frac{d u_{\varepsilon}}{d t}(t), \frac{d^{2} u_{\varepsilon}}{d t^{2}}(t)\right\rangle+\left\langle\frac{d u_{\varepsilon}}{d t}(t), \nabla V_{\varepsilon}\left(u_{\varepsilon}(t)\right)\right\rangle \\
& =\left\langle\frac{d u_{\varepsilon}}{d t}(t), \frac{d^{2} u_{\varepsilon}}{d t^{2}}(t)+\nabla V_{\varepsilon}\left(u_{\varepsilon}(t)\right)\right\rangle,
\end{aligned}
$$

e assim, de (3.71), temos que

$$
\frac{d}{d t}\left(\frac{1}{2}\left|\frac{d u_{\varepsilon}}{d t}(t)\right|^{2}+V_{\varepsilon}\left(u_{\varepsilon}(t)\right)\right)=\left\langle\frac{d u_{\varepsilon}}{d t}(t), 0\right\rangle=0, \quad \forall(\varepsilon, t) \in \mathbf{I} \times \mathbb{R} .
$$

Logo, para $\varepsilon \in \mathbf{I}$ fixado, temos que a função

$$
t \in \mathbb{R} \longmapsto \frac{1}{2}\left|\frac{d u_{\varepsilon}}{d t}(t)\right|^{2}+V_{\varepsilon}\left(u_{\varepsilon}(t)\right)
$$

é constante. Portanto, por (3.71), temos que

$$
\left|\frac{d u_{\varepsilon}}{d t}(t)\right|^{2}=2\left(\frac{1}{2}\left|v_{0_{\varepsilon}}\right|^{2}+V_{\varepsilon}\left(u_{0_{\varepsilon}}\right)-V_{\varepsilon}\left(u_{\varepsilon}(t)\right)\right), \quad \forall(\varepsilon, t) \in \mathbf{I} \times \mathbb{R} .
$$

Provaremos, a seguir, 2. e 3..

$\operatorname{De}\left(u_{0_{\varepsilon}}\right)_{\varepsilon},\left(v_{0_{\varepsilon}}\right)_{\varepsilon} \in\left(\mathcal{E}_{M}(\mathbb{R})\right)^{n}$ e $\left(V_{\varepsilon}\right)_{\varepsilon} \in \mathcal{E}_{\tau}\left[\mathbb{R}^{n} ; \mathbb{R}\right]$, existem $N \in \mathbb{N}, r_{1}>1$ e $\eta_{1} \in \mathbf{I}$ tais que

$$
\begin{gathered}
\left|u_{0_{\varepsilon}}\right| \leq r_{1} \varepsilon^{-N}, \quad \forall \varepsilon \in \mathbf{I}_{\eta_{1}} ; \\
\left|v_{0_{\varepsilon}}\right| \leq r_{1} \varepsilon^{-N}, \quad \forall \varepsilon \in \mathbf{I}_{\eta_{1}} ; \\
\left|V_{\varepsilon}(x)\right| \leq r_{1}(1+|x|)^{N} \varepsilon^{-N}, \quad \forall(\varepsilon, x) \in \mathbf{I}_{\eta_{1}} \times \mathbb{R}^{n} .
\end{gathered}
$$

Assim, de (3.73), (3.74), (3.75) e (3.76), temos que

$$
\left|\frac{d u_{\varepsilon}}{d t}(t)\right|^{2} \leq 2\left(\left|v_{0_{\varepsilon}}\right|^{2}+\left|V_{\varepsilon}\left(u_{0_{\varepsilon}}\right)\right|+\left|V_{\varepsilon}\left(u_{\varepsilon}(t)\right)\right|\right)
$$




$$
\begin{aligned}
& \leq 2\left(\left(r_{1} \varepsilon^{-N}\right)^{2}+r_{1}\left(1+\left|u_{0_{\varepsilon}}\right|\right)^{N} \varepsilon^{-N}+\left|V_{\varepsilon}\left(u_{\varepsilon}(t)\right)\right|\right) \\
& \leq 2 r_{1}^{2} \varepsilon^{-2 N}+2 r_{1}\left(\varepsilon^{-N}+r_{1} \varepsilon^{-N}\right)^{N} \varepsilon^{-N}+2\left|V_{\varepsilon}\left(u_{\varepsilon}(t)\right)\right| \\
& \leq 2 r_{1}^{2} \varepsilon^{-2 N}+2 r_{1}\left(1+r_{1}\right)^{N} \varepsilon^{-\left(N^{2}+2 N\right)}+2\left|V_{\varepsilon}\left(u_{\varepsilon}(t)\right)\right| \\
& \leq 4 r_{1}^{2}\left(1+r_{1}\right)^{N} \varepsilon^{-\left(N^{2}+2 N\right)}+2\left|V_{\varepsilon}\left(u_{\varepsilon}(t)\right)\right|
\end{aligned}
$$

para todo $(\varepsilon, t) \in \mathbf{I}_{\eta_{1}} \times \mathbb{R}$.

Sejam $N_{1}:=N^{2}+2 N$ e $c_{1}:=4 r_{1}^{2}\left(1+r_{1}\right)^{N}$. Então

$$
\left|\frac{d u_{\varepsilon}}{d t}(t)\right|^{2} \leq c_{1} \varepsilon^{-N_{1}}+2\left|V_{\varepsilon}\left(u_{\varepsilon}(t)\right)\right|, \quad \forall(\varepsilon, t) \mathbf{I}_{\eta_{1}} \times \mathbb{R} .
$$

Portanto,

$$
\left|\frac{d u_{\varepsilon}}{d t}(t)\right| \leq\left(c_{1} \varepsilon^{-N_{1}}+2\left|V_{\varepsilon}\left(u_{\varepsilon}(t)\right)\right|\right)^{1 / 2}, \quad \forall(\varepsilon, t) \in \mathbf{I}_{\eta_{1}} \times \mathbb{R},
$$

o que prova 2.

Para provarmos 3. notemos que

$$
u_{\varepsilon}(t)=u_{0_{\varepsilon}}+\int_{t_{0}}^{t} \frac{d u_{\varepsilon}}{d t}(s) d s, \quad \forall(\varepsilon, t) \in \mathbf{I}_{\eta_{1}} \times \mathbb{R} .
$$

Suponhamos, primeiramente, $\left(V_{\varepsilon}\right)_{\varepsilon} L^{\infty}$-tipo em $\mathcal{E}_{\tau}\left[\mathbb{R}^{n} ; \mathbb{R}\right]$. Então existem $N_{2} \geq N_{1}$, $r_{2} \geq c_{1}$ e $\eta_{2} \in \mathbf{I}$ com $\eta_{2} \leq \eta_{1}$ tais que

$$
\left|V_{\varepsilon}(x)\right| \leq r_{2} \varepsilon^{-N_{2}}, \quad \forall(\varepsilon, x) \in \mathbf{I}_{\eta_{2}} \times \mathbb{R}^{n}
$$

Seja $T \geq \max \left\{1,\left|t_{0}\right|\right\}$. De (3.74), (3.77), (3.78) e (3.79), temos que

$$
\begin{aligned}
\left|u_{\varepsilon}(t)\right| & \leq\left|u_{0_{\varepsilon}}\right|+\left|\int_{t_{0}}^{t}\right| \frac{d u_{\varepsilon}}{d t}(s)|d s| \\
& \leq r_{1} \varepsilon^{-N}+\left|\int_{t_{0}}^{t}\left(c_{1} \varepsilon^{-N_{1}}+2\left|V_{\varepsilon}\left(u_{\varepsilon}(t)\right)\right|\right)^{1 / 2} d s\right| \\
& \leq r_{2} \varepsilon^{-N_{2}}+\left|\int_{t_{0}}^{t}\left(r_{2} \varepsilon^{-N_{2}}+2\left(r_{2} \varepsilon^{-N_{2}}\right)\right)^{1 / 2} d s\right| \\
& \leq r_{2} \varepsilon^{-N_{2}}+\left|t-t_{0}\right|\left(3 r_{2} \varepsilon^{-N_{2}}\right)^{1 / 2} \\
& \leq r_{2} \varepsilon^{-N_{2}}+\left(|t|+\left|t_{0}\right|\right) 3 r_{2} \varepsilon^{-N_{2}} \\
& \leq r_{2} \varepsilon^{-N_{2}}+(T|t|+T) 3 r_{2} \varepsilon^{-N_{2}} \\
& \leq T(1+|t|) 3 r_{2} \varepsilon^{-N_{2}}+T(1+|t|) 3 r_{2} \varepsilon^{-N_{2}} \\
& \leq 6 T r_{2}(1+|t|)^{N_{2}} \varepsilon^{-N_{2}}
\end{aligned}
$$


para todo $(\varepsilon, t) \in \mathbf{I}_{\eta_{2}} \times \mathbb{R}$.

Seja $c_{2}:=6 \operatorname{Tr}_{2}$. Então

$$
\left|u_{\varepsilon}(t)\right| \leq c_{2}(1+|t|)^{N_{2}} \varepsilon^{-N_{2}}, \quad \forall(\varepsilon, t) \in \mathbf{I}_{\eta_{2}} \times \mathbb{R},
$$

o que prova 3. nesse caso.

Finalmente, suponhamos $V_{\varepsilon} \geq 0$ para todo $\varepsilon \in \mathbf{I}$. Então de (3.73), temos que

$$
\left|\frac{d u_{\varepsilon}}{d t}(t)\right|^{2} \leq 2\left(\frac{1}{2}\left|v_{0_{\varepsilon}}\right|^{2}+V_{\varepsilon}\left(u_{0_{\varepsilon}}\right)\right), \quad \forall(\varepsilon, t) \in \mathbf{I} \times \mathbb{R} .
$$

Logo, de (3.74), (3.75) e (3.76) temos, para todo $(\varepsilon, t) \in \mathbf{I}_{\eta_{1}} \times \mathbb{R}$, que

$$
\begin{aligned}
\left|\frac{d u_{\varepsilon}}{d t}(t)\right|^{2} & \leq 2\left(\left|v_{0_{\varepsilon}}\right|^{2}+\left|V_{\varepsilon}\left(u_{0_{\varepsilon}}\right)\right|\right) \\
& \leq 2\left(\left(r_{1} \varepsilon^{-N}\right)^{2}+r_{1}\left(1+\left|u_{0_{\varepsilon}}\right|\right)^{N} \varepsilon^{-N}\right) \\
& \leq 2 r_{1}^{2} \varepsilon^{-2 N}+2 r_{1}\left(\varepsilon^{-N}+r_{1} \varepsilon^{-N}\right)^{N} \varepsilon^{-N} \\
& \leq 2 r_{1}^{2} \varepsilon^{-2 N}+2 r_{1}\left(1+r_{1}\right)^{N} \varepsilon^{-\left(N^{2}+2 N\right)} \\
& \leq 4 r_{1}^{2}\left(1+r_{1}\right)^{N} \varepsilon^{-\left(N^{2}+2 N\right)} .
\end{aligned}
$$

Sejam $N_{3}:=N^{2}+2 N, r_{3}:=4 r_{1}^{2}\left(1+r_{1}\right)^{N}$. Então

$$
\left|\frac{d u_{\varepsilon}}{d t}(t)\right|^{2} \leq r_{3} \varepsilon^{-N_{3}}, \quad \forall(\varepsilon, t) \in \mathbf{I}_{\eta_{1}} \times \mathbb{R},
$$

e assim,

$$
\left|\frac{d u_{\varepsilon}}{d t}(t)\right| \leq\left(r_{3} \varepsilon^{-N_{3}}\right)^{1 / 2} \leq r_{3} \varepsilon^{-N_{3}}, \quad \forall(\varepsilon, t) \in \mathbf{I}_{\eta_{1}} \times \mathbb{R} .
$$

Seja $T \geq \max \left\{1,\left|t_{0}\right|\right\}$. De (3.74), (3.77) e (3.80) temos

$$
\begin{aligned}
\left|u_{\varepsilon}(t)\right| & \leq\left|u_{0_{\varepsilon}}\right|+\left|\int_{t_{0}}^{t}\right| \frac{d u_{\varepsilon}}{d t}(s)|d s| \\
& \leq r_{1} \varepsilon^{-N_{1}}+\left|\int_{t_{0}}^{t} r_{3} \varepsilon^{-N_{3}} d s\right| \\
& \leq r_{3} \varepsilon^{-N_{3}}+\left|t-t_{0}\right| r_{3} \varepsilon^{-N_{3}} \\
& \leq r_{3} \varepsilon^{-N_{3}}+\left(|t|+\left|t_{0}\right|\right) r_{3} \varepsilon^{-N_{3}} \\
& \leq r_{3} \varepsilon^{-N_{3}}+(T|t|+T) r_{3} \varepsilon^{-N_{3}} \\
& \leq T(1+|t|) r_{3} \varepsilon^{-N_{3}}+T(1+|t|) r_{3} \varepsilon^{-N_{3}} \\
& \leq 2 T r_{3}(1+|t|)^{N_{3}} \varepsilon^{-N_{3}},
\end{aligned}
$$


para todo $(\varepsilon, t) \in \mathbf{I}_{\eta_{3}} \times \mathbb{R}$.

Sejam $c_{3}:=2 T r_{3}$ e $\eta_{3}:=\eta_{1}$. Então

$$
\left|u_{\varepsilon}(t)\right| \leq c_{3}(1+|t|)^{N_{3}} \varepsilon^{-N_{3}}, \quad \forall(\varepsilon, t) \in \mathbf{I}_{\eta_{3}} \times \mathbb{R},
$$

o que prova 3. nesse caso.

Das proposições anteriores temos o seguinte teorema de existência.

Teorema 3.2.5 Seja $V \in \mathcal{G}_{\tau}\left(\mathbb{R}^{n} ; \mathbb{R}\right)$ tal que $V$ é $L^{\infty}$-tipo em $\mathcal{G}_{\tau}\left(\mathbb{R}^{n} ; \mathbb{R}\right)$ ou existe um representante $\left(V_{\varepsilon}\right)_{\varepsilon}$ de $V$ de modo que $V_{\varepsilon} \geq 0$, para todo $\varepsilon \in \mathbf{I}$. Então o problema (3.61) tem uma solução em $\mathcal{G}\left(\mathbb{R} ; \mathbb{R}^{n}\right)$ e também tem uma solução em $\mathcal{G}_{\tau}\left(\mathbb{R} ; \mathbb{R}^{n}\right)$.

Demonstração: Seja $\left(V_{\varepsilon}\right)_{\varepsilon} \in \mathcal{E}_{\tau}\left[\mathbb{R}^{n} ; \mathbb{R}\right]$ um representante de $V$ tal que $\left(V_{\varepsilon}\right)_{\varepsilon}$ é $L^{\infty}$-tipo em $\mathcal{E}_{\tau}\left[\mathbb{R}^{n} ; \mathbb{R}\right]$, ou de modo que $V_{\varepsilon} \geq 0$, para todo $\varepsilon \in \mathbf{I}$. Considere $\left(u_{0_{\varepsilon}}\right)_{\varepsilon}$ e $\left(v_{0_{\varepsilon}}\right)_{\varepsilon}$ representantes de $u_{0}$ e $v_{0}$, respectivamente.

Fixe $\varepsilon \in \mathbf{I}$. Então, da Proposição 3.2.4, existe $u_{\varepsilon} \in\left(\mathscr{C}^{\infty}\left(\mathbb{R} ; \mathbb{R}^{n}\right)\right)^{\mathbf{I}}$ satisfazendo (3.71). Assim, da Proposição 3.2.2 e da Proposição 3.2.4, temos que $\left(u_{\varepsilon}\right)_{\varepsilon} \in \mathcal{E}_{M}\left[\mathbb{R} ; \mathbb{R}^{n}\right]$ e $\left(u_{\varepsilon}\right)_{\varepsilon} \in \mathcal{E}_{\tau}\left[\mathbb{R} ; \mathbb{R}^{n}\right]$. Portanto, $\left(u_{\varepsilon}\right)_{\varepsilon}+\mathcal{N}\left[\mathbb{R} ; \mathbb{R}^{n}\right]$ é uma solução de $(3.61)$ em $\mathcal{G}\left(\mathbb{R} ; \mathbb{R}^{n}\right)$, e $\left(u_{\varepsilon}\right)_{\varepsilon}+\mathcal{N}_{\tau}\left[\mathbb{R} ; \mathbb{R}^{n}\right]$ é uma solução de $(3.61)$ em $\mathcal{G}_{\tau}\left(\mathbb{R} ; \mathbb{R}^{n}\right)$.

No Exemplo 3.2.6 iremos substituir função a $V$ pela função $i_{\mathcal{S}^{\prime}}(\delta)$ (Exemplo 2.3.4). Note que $\left(i_{\mathcal{S}^{\prime}}(\delta)\right)^{\prime \prime}$ não é $L^{\infty}$-ln-tipo em $\mathcal{E}_{\tau}[\mathbb{R} ; \mathbb{R}]$ (Exemplo 3.1.6).

Exemplo 3.2.6 Sejam $\delta$ a distribuição temperada definida no Exemplo 2.1.19, $i_{\mathcal{S}^{\prime}}$ como no Teorema 2.3 .3 e $\left(u_{0}, v_{0}\right) \in \overline{\mathbb{R}}^{n} \times \overline{\mathbb{R}}^{n}$. Então o problema

$$
\begin{aligned}
& \frac{d^{2} u}{d t^{2}}+\nabla i_{\mathcal{S}^{\prime}}(\delta) \circ u=0 \\
& \left(u\left(t_{0}\right), \frac{d u}{d t}\left(t_{0}\right)\right)=\left(u_{0}, v_{0}\right)
\end{aligned}
$$

tem solução em $\mathcal{G}(\mathbb{R} ; \mathbb{R})$ e em $\mathcal{G}_{\tau}(\mathbb{R} ; \mathbb{R})$. 
De fato, a existência de solução decorre diretamente do Teorema 3.2 .5 (substituindo $V$ por $\left.i_{\mathcal{S}^{\prime}}(\delta) \in \mathcal{G}_{\tau}(\mathbb{R} ; \mathbb{R})\right)$, observando que $\left(\rho_{\varepsilon}\right)_{\varepsilon}$ é $L^{\infty}$-tipo em $\mathcal{E}_{\tau}[\mathbb{R} ; \mathbb{R}]$ e é um representante de $i_{\mathcal{S}^{\prime}}(\delta)$ (Exemplo 3.1.3 e Exemplo 2.3.4).

Recentemente S. Konjik, M. Kunzinger e M. Oberguggenberger apresentam em [KKO] um estudo sobre o cálculo de variações no contexto das funções generalizadas de Colombeau. Nesse trabalho eles estudam, de um modo geral, as equações de Euler-Lagrange.

\subsection{Solução para frente em sistemas autônomos}

Nesta seção estudaremos, sob que condições, os sistemas autonômos da forma

$$
\begin{aligned}
& \frac{d u}{d t}=F \circ u \\
& u\left(t_{0}\right)=x_{0}
\end{aligned},
$$

onde $t_{0} \in \mathbb{R}, x_{0} \in \overline{\mathbb{R}}^{n}$ e $F \in \mathcal{G}_{\tau}\left(\mathbb{R}^{n} ; \mathbb{R}^{n}\right)$, admitem soluções em $\mathcal{G}_{\tau}\left(\left[t_{0},+\infty\left[; \mathbb{R}^{n}\right)\right.\right.$ e em $\mathcal{G}\left(\left[t_{0},+\infty\left[; \mathbb{R}^{n}\right)\right.\right.$. Nos resultados que apresentaremos a função $F \in \mathcal{G}_{\tau}\left(\mathbb{R}^{n} ; \mathbb{R}^{n}\right)$ terá a seguinte propriedade:

Definição 3.3.1 Dizemos que $F \in \mathcal{G}_{\tau}\left(\mathbb{R}^{n} ; \mathbb{R}^{n}\right)$ é dissipativa em $\mathcal{G}_{\tau}\left(\mathbb{R}^{n} ; \mathbb{R}^{n}\right)$ se, existe um representante $\left(F_{\varepsilon}\right)_{\varepsilon}$ de $F$ tal que

$$
\left\langle x-y, F_{\varepsilon}(x)-F_{\varepsilon}(y)\right\rangle \leq 0, \quad \forall(\varepsilon, x, y) \in \mathbf{I} \times \mathbb{R}^{n} \times \mathbb{R}^{n},
$$

onde $\langle$,$\rangle denota o produto interno usual de \mathbb{R}^{n}$.

Exemplo 3.3.2 Sejam $f \in \mathcal{O}_{M}(\mathbb{R} ; \mathbb{R})$ não-crescente, $g \in \mathcal{O}_{M}(\mathbb{R} ; \mathbb{R})$ não-decrescente $e$ $h: \varepsilon \in \mathbf{I} \longrightarrow \mathbb{R}$ tal que $h \geq 0$ e existem $N_{1} \in \mathbb{N}^{*}, c_{1}>0$ tais que

$$
|h(\varepsilon)| \leq c_{1} \varepsilon^{-N_{1}}, \quad \forall \varepsilon \in \mathbf{I} .
$$


Considere as familias $\left(u_{\varepsilon}\right)_{\varepsilon},\left(v_{\varepsilon}\right)_{\varepsilon} \in\left(\mathscr{C}^{\infty}(\mathbb{R} ; \mathbb{R})\right)^{\mathbf{I}} e\left(w_{\varepsilon}\right)_{\varepsilon} \in \mathscr{C}_{c}^{\infty}\left(\mathbb{R}^{2} ; \mathbb{R}^{2}\right)$ definidas por

$$
\begin{aligned}
u_{\varepsilon}(x) & :=h(\varepsilon) f(x) ; \\
v_{\varepsilon}(x) & :=g(-h(\varepsilon) x) ; \\
w_{\varepsilon}(x, y) & :=\left(u_{\varepsilon}(x), v_{\varepsilon}(y)\right) .
\end{aligned}
$$

\section{Então}

1. $u:=\left[\left(u_{\varepsilon}\right)_{\varepsilon}\right]$ é dissipativa em $\mathcal{G}_{\tau}(\mathbb{R} ; \mathbb{R})$;

2. $v:=\left[\left(v_{\varepsilon}\right)_{\varepsilon}\right]$ é dissipativa em $\mathcal{G}_{\tau}(\mathbb{R} ; \mathbb{R})$;

3. $w:=\left[\left(w_{\varepsilon}\right)_{\varepsilon}\right]$ é dissipativa em $\mathcal{G}_{\tau}\left(\mathbb{R}^{2} ; \mathbb{R}^{2}\right)$.

De fato, observe que, se $f, g \in \mathcal{O}_{M}(\mathbb{R} ; \mathbb{R})$, então, para todo $\alpha \in \mathbb{N}^{n}$, existem $N_{2} \in \mathbb{N}$ com $N_{2}>N_{1}$ e $c_{2}>c_{1}$ tais que

$$
\begin{aligned}
& \left|\partial^{\alpha} f(x)\right| \leq c_{2}(1+|x|)^{N_{2}}, \quad \forall x \in \mathbb{R} ; \\
& \left|\partial^{\alpha} g(x)\right| \leq c_{2}(1+|x|)^{N_{2}}, \quad \forall x \in \mathbb{R} .
\end{aligned}
$$

Para 1. notemos que, para todo $(\varepsilon, x) \in I \times \mathbb{R}$,

$$
\begin{aligned}
\left|\partial^{\alpha} u_{\varepsilon}(x)\right| & \leq\left|\partial^{\alpha}(h(\varepsilon) f(x))\right| \\
& \leq|h(\varepsilon)|\left|\partial^{\alpha} f(x)\right| \\
& \leq c_{1} \varepsilon^{-N_{1}} c_{2}(1+|x|)^{N_{2}} \\
& \leq c_{1} c_{2}(1+|x|)^{N_{2}} \varepsilon^{-N_{1}} \\
& \leq c_{3}(1+|x|)^{N_{2}} \varepsilon^{-N_{2}},
\end{aligned}
$$

onde $c_{3}:=c_{1} c_{2}$. Logo, $\left(u_{\varepsilon}\right)_{\varepsilon} \in \mathcal{E}_{\tau}[\mathbb{R} ; \mathbb{R}]$. Fixe $\varepsilon \in \mathbf{I}$ e observe que

$$
\begin{aligned}
\left\langle x-y, u_{\varepsilon}(x)-u_{\varepsilon}(y)\right\rangle & =\langle x-y, h(\varepsilon) f(x)-h(\varepsilon) f(y)\rangle \\
& =\langle x-y, h(\varepsilon)(f(x)-f(y))\rangle .
\end{aligned}
$$


Como $h \geq 0$ e $f$ é não-crescente então $\left\langle x-y, u_{\varepsilon}(x)-u_{\varepsilon}(y)\right\rangle \leq 0$. Como $\varepsilon \in \mathbf{I}$ é arbitrário, temos que $u:=\left[\left(u_{\varepsilon}\right)_{\varepsilon}\right]$ é dissipativa em $\mathcal{G}_{\tau}(\mathbb{R} ; \mathbb{R})$. Para 2. notemos que, para todo $(\varepsilon, x) \in \mathbf{I} \times \mathbb{R}$,

$$
\begin{aligned}
\left|\partial^{\alpha} v_{\varepsilon}(x)\right| & \leq\left|\partial^{\alpha} g(-h(\varepsilon) x)\right| \\
& \leq c_{2}(1+|-h(\varepsilon) x|)^{N_{2}} \\
& \leq c_{2}(1+|h(\varepsilon)||x|)^{N_{2}} \\
& \leq c_{2}\left(1+c_{1} \varepsilon^{-N_{1}}|x|\right)^{N_{2}} \\
& \leq c_{2}\left(c_{1} \varepsilon^{-N_{1}}+c_{1} \varepsilon^{-N_{1}}|x|\right)^{N_{2}} \\
& \leq c_{2}\left(c_{1} \varepsilon^{-N_{1}}\right)^{N_{2}}(1+|x|)^{N_{2}} \\
& \leq c_{1}^{N_{2}} c_{2}(1+|x|)^{N_{1} N_{2}} \varepsilon^{-N_{1} N_{2}} \\
& \leq c_{4}(1+|x|)^{N_{3}} \varepsilon^{-N_{3}},
\end{aligned}
$$

onde $c_{4}:=c_{1}^{N_{2}} c_{2}$ e $N_{3}:=N_{1} N_{2}$. Logo, $\left(v_{\varepsilon}\right)_{\varepsilon} \in \mathcal{E}_{\tau}[\mathbb{R} ; \mathbb{R}]$. Fixe $\varepsilon \in \mathbf{I}$ e observe que

$$
\left\langle x-y, v_{\varepsilon}(x)-v_{\varepsilon}(y)\right\rangle=\langle x-y, g(-h(\varepsilon) x)-g(-h(\varepsilon) y)\rangle
$$

Como $g$ é não-decrescente então $g(-h(\varepsilon) \bullet)$ é não-crescente e assim, $\left\langle x-y, v_{\varepsilon}(x)-v_{\varepsilon}(y)\right\rangle \leq 0$. Como $\varepsilon \in \mathbf{I}$ é arbitrário, temos que $v:=\left[\left(v_{\varepsilon}\right)_{\varepsilon}\right]$ é dissipativa em $\mathcal{G}_{\tau}(\mathbb{R} ; \mathbb{R})$. Finalmente, para 3. notemos que, de 1. e 2., tem-se $\left(w_{\varepsilon}\right)_{\varepsilon} \in \mathcal{E}_{\tau}\left(\mathbb{R}^{2} ; \mathbb{R}^{2}\right)$ e $w:=\left[\left(w_{\varepsilon}\right)_{\varepsilon}\right]$ é dissipativa em $\mathcal{G}_{\tau}\left(\mathbb{R}^{2} ; \mathbb{R}^{2}\right)$.

Para estudarmos o problema (3.81) apresentaremos, como foi feito no parágrafo anterior, alguns resultados a nível de representantes em $\mathcal{E}_{\tau}\left[\left[t_{0},+\infty\left[; \mathbb{R}^{n}\right]\right.\right.$ e em $\mathcal{E}_{M}\left[\left[t_{0},+\infty\left[; \mathbb{R}^{n}\right]\right.\right.$. Como $F \in \mathcal{G}_{\tau}\left(\mathbb{R}^{n} ; \mathbb{R}^{n}\right)$ será dissipativa, necessitaremos de resultados na teoria clássica das Equações Diferenciais Ordinárias para funções com a propriedade (3.82) ([SC], [Tan]). Para facilitar a leitura, e por esses resultados serem fundamentais para o nosso estudo, optamos por enunciá-los e demonstrá-los nesta seção. É isso que faremos nos próximos três resultados. 
Proposição 3.3.3 Sejam $a \in \mathbb{R}, \quad w:] a,+\infty[\times \mathbb{R} \longrightarrow[0,+\infty[$ contínua, $f:] a,+\infty\left[\times \mathbb{R}^{n} \longrightarrow \mathbb{R}^{n}\right.$ contínua e $\left.g:\right] a,+\infty[\times \mathbb{R} \longrightarrow \mathbb{R}$ definida por

$$
g(t, s)=s w(t, s)
$$

tais que

(i) $g(t, \bullet)$ é nãa-decrescente em $\mathbb{R}, \quad \forall t>a$;

(ii) $\langle x, f(t, x)\rangle \leq|x| w(t,|x|), \quad \forall(t, x) \in] a,+\infty\left[\times \mathbb{R}^{n}\right.$

(iii) para todo $\left.\left(s_{0}, y_{0}\right) \in\right] a,+\infty\left[\times \mathbb{R}\right.$, existem $\delta_{1}>0$ e $v$ definida em $] s_{0}-\delta_{1},+\infty[$ tais que $] s_{0}-\delta_{1},+\infty[\subset] a,+\infty[$, v é derivável em $] s_{0}-\delta_{1},+\infty[e$

$$
\frac{d v}{d t}(t)=w(t, v(t)), \quad \forall t>s_{0} \quad e \quad v\left(s_{0}\right)=y_{0}
$$

$\left.S e\left(t_{0}, x_{0}\right) \in\right] a,+\infty\left[\times \mathbb{R}^{n}\right.$ e o problema

$$
\begin{aligned}
& \frac{d u}{d t}(t)=f(t, u(t)) \\
& u\left(t_{0}\right)=x_{0}
\end{aligned}
$$

tem solução maximal, então o domínio dessa solução contém o intervalo $] t_{0}-\delta,+\infty[$ para $\operatorname{algum} \delta>0$.

Demonstração: Sejam $\left.\left(t_{0}, x_{0}\right) \in\right] a,+\infty\left[\times \mathbb{R}^{n}, J\right.$ intervalo aberto de $] a,+\infty\left[\operatorname{com} t_{0} \in J\right.$ e $u: J \longrightarrow \mathbb{R}$ solução maximal de (3.83).

Como $t_{0} \in J$ e $J$ é aberto existe $\delta>0$ tal que $] t_{0}-\delta, t_{0}+\delta[\subset J$. Se $J$ não for limitado superiormente é claro que $] t_{0}-\delta,+\infty[\subset J$. Suponhamos, por absurdo, $J$ limitado superiormente por $b>0$, isto é $] t_{0}-\delta,+\infty[\cap J=] t_{0}-\delta, b[$. Provaremos que $u$ é limitada em $] t_{0}, b[$ e assim teremos uma contradição ([Chi], Theorem 1.186).

De (3.83) temos que

$$
\left.\langle u(t), f(t, u(t))\rangle=\left(u(t), \frac{d u}{d t}(t)\right\rangle=\frac{1}{2} \frac{d}{d t}\left(|u(t)|^{2}\right), \quad \forall t \in\right] t_{0}-\delta, b[.
$$


Assim

$$
\int_{t_{0}}^{t} 2\langle u(s), f(s, u(s))\rangle d s=\int_{t_{0}}^{t} \frac{d|u|^{2}}{d t}(s) d s=|u(t)|^{2}-\left|u\left(t_{0}\right)\right|^{2}
$$

ou seja

$$
\left.|u(t)|^{2}=\left|u\left(t_{0}\right)\right|^{2}+2 \int_{t_{0}}^{t}\langle u(s), f(s, u(s))\rangle d s, \quad \forall t \in\right] t_{0}, b[.
$$

De (3.83) e (ii) obtemos que

$$
|u(t)|^{2} \leq\left|u\left(t_{0}\right)\right|^{2}+2 \int_{t_{0}}^{t}|u(s)| w(s,|u(s)|) d s
$$

e assim

$$
\left.|u(t)|^{2}<1+\left|u\left(t_{0}\right)\right|^{2}+2 \int_{t_{0}}^{t}|u(s)| w(s,|u(s)|) d s, \quad \forall t \in\right] t_{0}, b[.
$$

Por $($ iii $)$, existem $\delta_{1}>0$ e $v$ definida em $] t_{0}-\delta_{1},+\infty[\subset] a,+\infty[$ tais que $v$ é derivável

e

$$
\frac{d v}{d t}(t)=w(t, v(t)), \quad \forall t>t_{0} \quad \text { e } \quad v\left(t_{0}\right)=\sqrt{1+\left|u\left(t_{0}\right)\right|^{2}}
$$

Logo

$$
\frac{1}{2} \frac{d}{d t}\left(v^{2}(t)\right)=v(t) \frac{d v}{d t}(t)=v(t) w(t, v(t)), \quad \forall t>t_{0}
$$

Portanto

$$
v^{2}(t)-v^{2}\left(t_{0}\right)=2 \int_{t_{0}}^{t} \frac{d v^{2}}{d t}(s) d s=2 \int_{t_{0}}^{t} v(s) w(s, v(s)) d s, \quad \forall t>t_{0},
$$

ou seja

$$
v^{2}(t)=1+\left|u\left(t_{0}\right)\right|^{2}+2 \int_{t_{0}}^{t} v(s) w(s, v(s)) d s, \quad \forall t>t_{0} .
$$

Como $v^{2}\left(t_{0}\right)=1+\left|u\left(t_{0}\right)\right|^{2}>\left|u\left(t_{0}\right)\right|^{2}, v\left(t_{0}\right)>0$ e $u$ e $v$ são contínuas em $t_{0}$, existe $0<\delta_{2}<\delta_{1}$ tal que

$$
] t_{0}, t_{0}+\delta_{2}[\subset] t_{0}, b\left[, \quad v(t)>0 \quad \text { e } \quad v^{2}(t)>|u(t)|^{2}, \quad \forall t \in\right] t_{0}-\delta_{2}, t_{0}+\delta_{2}[,
$$

e assim

$$
v(t)=|v(t)|>|u(t)|, \quad \forall t \in\left[t_{0}, t_{0}+\delta_{2}[.\right.
$$

Provaremos que

$$
v(t)>|u(t)|, \quad \forall t \in\left[t_{0}, b[.\right.
$$


Suponhamos, por absurdo, (3.89) falsa, isto é, que exista $t_{1} \in\left[t_{0}, b[\right.$ com $v\left(t_{1}\right) \leq\left|u\left(t_{1}\right)\right|$. Como $v$ e $|u|$ são contínuas e (3.88) é verdadeira temos que o conjunto

$$
A:=\{s \in] t_{0}, b[|v(s)=| u(s) \mid\}
$$

é não vazio e limitado inferiormente por $t_{0}+\delta_{2}$.

Seja $t_{2}=\min A$. Note que, por (3.87), tem-se

$$
\left|u\left(t_{2}\right)\right|^{2}=v^{2}\left(t_{2}\right)=1+\left|u\left(t_{0}\right)\right|^{2}+2 \int_{t_{0}}^{t_{2}} v(s) w(s, v(s)) d s
$$

e que, por (3.88) e da continuidade de $v-|u|$ tem-se

$$
v(t)>|u(t)|, \quad \forall t \in\left[t_{0}, t_{2}[\right.
$$

De $(i),(3.85),(3.90)$ e (3.91) tem-se

$$
\begin{aligned}
\left|u\left(t_{2}\right)\right|^{2} & =1+\left|u\left(t_{0}\right)\right|^{2}+2 \int_{t_{0}}^{t_{2}} v(s) w(s, v(s)) d s \\
& \geq 1+\left|u\left(t_{0}\right)\right|^{2}+2 \int_{t_{0}}^{t_{2}}|u(s)| w(s,|u(s)|) d s \\
& >\left|u\left(t_{2}\right)\right|^{2}
\end{aligned}
$$

o que é um absurdo. Portanto (3.89) é verdadeira.

Da continuidade de $v$ em $\left[t_{0}, b\right]$, existe $M>0$ tal que $|v(t)| \leq M$, para $t \in\left[t_{0}, b\right]$. Logo, por (3.89), temos

$$
|u(t)|<v(t)=|v(t)| \leq M, \quad \forall t \in\left[t_{0}, b[,\right.
$$

que é o que queríamos provar.

Proposição 3.3.4 Sejam $\left.a \in \mathbb{R}, t_{0} \in\right] a,+\infty\left[, x_{0} \in \mathbb{R}^{n}\right.$ e $f \in \mathscr{C}^{\infty}(] a,+\infty\left[\times \mathbb{R}^{n} ; \mathbb{R}^{n}\right)$ tais que

(i) $\langle x-y, f(t, x)-f(t, y)\rangle \leq 0, \quad \forall(t, x, y) \in] a,+\infty\left[\times \mathbb{R}^{n} \times \mathbb{R}^{n}\right.$. 
Então o problema

$$
\begin{aligned}
& \frac{d u}{d t}(t)=f(t, u(t)) \\
& u\left(t_{0}\right)=x_{0}
\end{aligned}
$$

admite solução maximal u cujo domínio contém $] t_{0}-\delta,+\infty[$ para algum $\delta>0$. Além disso, $u \in \mathscr{C}^{\infty}\left(\left[t_{0},+\infty\left[; \mathbb{R}^{n}\right)\right.\right.$.

Demonstração: De $f$ pertencer a $\mathscr{C}^{\infty}(] a,+\infty\left[\times \mathbb{R}^{n} ; \mathbb{R}^{n}\right)$ temos que (3.92) admite solução maximal $u$ cujo domínio é um intervalo aberto $J$ ([Chi], Theorem 1.184).

Como $t_{0} \in J$ e $J$ é um aberto existe $\delta>0$ tal que $] t_{0}-\delta, t_{0}+\delta[\subset J$. Provaremos que ]$t_{0},+\infty[\subset J$. Para essa finalidade utilizaremos a Proposição 3.3.3.

Sejam $w:] a,+\infty[\times \mathbb{R} \longrightarrow[0,+\infty[$ e $g:] a,+\infty[\times \mathbb{R} \longrightarrow \mathbb{R}$ as funções definidas por

$$
\begin{gathered}
w(t, s)=|f(t, 0)| \\
g(t, s)=s w(t, s)=s|f(t, 0)| .
\end{gathered}
$$

É claro que $g(t, \bullet)$ é não-decrescente em $\mathbb{R}$, para todo $t>a$. Além disso temos, de $(i)$, que

$$
\begin{aligned}
\langle x, f(t, x)\rangle & =\langle x-0, f(t, x)-f(t, 0)+f(t, 0)\rangle \\
& =\langle x, f(t, x)-f(t, 0)\rangle+\langle x, f(t, 0)\rangle \\
& \leq\langle x, f(t, 0)\rangle \\
& \leq|x||f(t, 0)|=|x| w(t,|x|),
\end{aligned}
$$

para todo $(t, x) \in] a,+\infty\left[\times \mathbb{R}^{n}\right.$, ou seja a afirmação $(i i)$ da Proposição 3.3.3 está satisfeita.

Notemos ainda que, para todo $\left.\left(s_{0}, y_{0}\right) \in\right] a,+\infty[\times \mathbb{R}$, a função

$$
v(t)=y_{0}+\int_{s_{0}}^{t}|f(s, 0)| d s \quad \forall t>a
$$

é tal que $v$ é derivável em $] a,+\infty\left[, \quad v\left(s_{0}\right)=y_{0} \quad \mathrm{e}\right.$

$$
\frac{d v}{d t}(t)=|f(t, 0)|=w(t, v(t)), \quad \forall t>s_{0},
$$

ou seja a afirmação (iii) da Proposição 3.3 .3 está satisfeita. Portanto, pela Proposição 3.3.3, existe $\delta>0$ tal que $] t_{0}-\delta,+\infty[\subset J$. 
De (3.92) temos que $u \in \mathscr{C}^{\infty}(] t_{0}-\delta,+\infty\left[; \mathbb{R}^{n}\right)$. Logo $u \in \mathscr{C}^{\infty}\left(\left[t_{0},+\infty\left[; \mathbb{R}^{n}\right)\right.\right.$.

Proposição 3.3.5 Sejam $\left.a \in \mathbb{R}, t_{0} \in\right] a,+\infty\left[, x_{0} \in \mathbb{R}^{n}\right.$ e $f \in \mathscr{C}^{\infty}(] a,+\infty\left[\times \mathbb{R}^{n} ; \mathbb{R}^{n}\right)$ tais que

(i) $\langle x-y, f(t, x)-f(t, y)\rangle \leq 0, \quad \forall(t, x, y) \in] a,+\infty\left[\times \mathbb{R}^{n} \times \mathbb{R}^{n}\right.$

Então existe $u \in \mathscr{C}^{\infty}\left(\left[t_{0},+\infty\left[; \mathbb{R}^{n}\right)\right.\right.$ tal que

1. $\frac{d u}{d t}(t)=f(t, u(t)), \quad \forall t \geq t_{0} \quad e \quad u\left(t_{0}\right)=x_{0}$;

2. $\left|u(t)-x_{0}\right| \leq \int_{t_{0}}^{t}\left|f\left(s, x_{0}\right)\right| d s, \quad \forall t \geq t_{0}$.

Demonstração: Da Proposição 3.3.4, existem $\delta>0$ e $u \in \mathscr{C}^{\infty}(] t_{0}-\delta,+\infty\left[; \mathbb{R}^{n}\right)$ satisfazendo 1 ..

Sejam $h:]-\delta,+\infty\left[\times \mathbb{R}^{n} \longrightarrow \mathbb{R}^{n}\right.$ e $\left.v:\right]-\delta,+\infty\left[\longrightarrow \mathbb{R}^{n}\right.$ definidas por

$$
h(t, x)=f\left(t+t_{0}, x+x_{0}\right) \quad ; \quad v(t)=u\left(t+t_{0}\right)-x_{0} .
$$

Então, de 1., temos que

$$
\frac{d v}{d t}(t)=\frac{d u}{d t}\left(t+t_{0}\right)=f\left(t+t_{0}, u\left(t+t_{0}\right)\right)=f\left(t+t_{0}, v(t)+x_{0}\right)=h(t, v(t)), \quad \forall t \geq 0,
$$

e assim

$$
\frac{d v}{d t}(t)=h(t, v(t)), \quad \forall t \geq 0 \quad \text { e } \quad v(0)=0
$$

Além disso, de $(i)$, temos que

$$
\langle x-y, h(t, x)-h(t, y)\rangle \leq 0, \quad \forall(t, x) \in\left[0,+\infty\left[\times \mathbb{R}^{n}\right.\right.
$$

De (3.93) e (3.94) temos que

$$
\begin{aligned}
\frac{1}{2} \frac{d}{d t}\left(|v(t)|^{2}\right) & =\left\langle v(t), \frac{d v}{d t}(t)\right\rangle \\
& =\langle v(t), h(t, v(t))\rangle
\end{aligned}
$$




$$
\begin{aligned}
& =\langle v(t)-v(0), h(t, v(t))-h(t, v(0))+h(t, v(0))\rangle \\
& =\langle v(t)-v(0), h(t, v(t))-h(t, v(0))\rangle+\langle v(t), h(t, 0)\rangle \\
& \leq\langle v(t), h(t, 0)\rangle \\
& \leq|v(t)||h(t, 0)|
\end{aligned}
$$

para todo $t \geq 0$.

Seja $\alpha:\left[0,+\infty\left[\longrightarrow \mathbb{R}\right.\right.$ dada por $\alpha(t)=|v(t)|^{2}$. Então, das contas anteriores, temos que

$$
\frac{1}{2} \frac{d \alpha}{d t}(t) \leq \sqrt{\alpha(t)}|h(t, 0)|, \quad \forall t \geq 0
$$

Seja $\left.t_{1} \in\right] 0,+\infty\left[\right.$ e suponhamos $\alpha\left(t_{1}\right) \neq 0$. Da continuidade de $\alpha$, existe $t_{2} \geq 0$ tal que $\alpha\left(t_{2}\right)=0$ e $\alpha(t)>0$ para $\left.\left.T \in\right] t_{2}, t_{1}\right]$, pois, basta definir $t_{2}:=\sup \left\{s \in\left[0, t_{1}[\mid \alpha(s)=0\}\right.\right.$. Como $\alpha$ nunca se anula em $] t_{2}, t_{1}[$ temos, de (3.95), que

$$
\left.\frac{1}{2} \frac{1}{\sqrt{\alpha(t)}} \frac{d \alpha}{d t}(t) \leq|h(t, 0)|, \quad \forall t \in\right] t_{2}, t_{1}[.
$$

Logo

$$
\lim _{a \rightarrow t_{2}^{+}} \int_{a}^{t_{1}} \frac{1}{2} \frac{1}{\sqrt{\alpha(s)}} \frac{d \alpha}{d t}(s) d s \leq \int_{0}^{t_{1}}|h(s, 0)| d s
$$

e assim

$$
\sqrt{\alpha\left(t_{1}\right)}=\sqrt{\alpha\left(t_{1}\right)}-\sqrt{\alpha\left(t_{2}\right)}=\lim _{a \rightarrow t_{2}^{+}}\left(\sqrt{\alpha\left(t_{1}\right)}-\sqrt{\alpha(a)}\right) \leq \int_{0}^{t_{1}}|h(s, 0)| d s
$$

isto é

$$
\left|v\left(t_{1}\right)\right| \leq \int_{0}^{t_{1}}|h(s, 0)| d s=\int_{0}^{t_{1}}\left|f\left(s+t_{0}, x_{0}\right)\right| d s=\int_{t_{0}}^{t_{1}+t_{0}}\left|f\left(\tau, x_{0}\right)\right| d \tau
$$

É claro que (3.96) é verdadeira se $\alpha\left(t_{1}\right)=0$. Dessa forma tem-se que

$$
|v(t)| \leq \int_{t_{0}}^{t+t_{0}}\left|f\left(\tau, x_{0}\right)\right| d \tau, \quad \forall t \geq 0
$$

e assim

$$
\left|u(t)-x_{0}\right|=\left|v\left(t-t_{0}\right)\right| \leq \int_{t_{0}}^{t}\left|f\left(s, x_{0}\right)\right| d s, \quad \forall t \geq t_{0},
$$

o que prova 2. 
Como nesta seção estudaremos sistemas autonômos, convém observar que, a partir dos resultados anteriores, temos, para esses sistemas, o seguinte.

Teorema 3.3.6 Sejam $\left.a \in \mathbb{R}, t_{0} \in\right] a,+\infty\left[, x_{0} \in \mathbb{R}^{n}\right.$ e $F \in \mathscr{C}^{\infty}\left(\mathbb{R}^{n} ; \mathbb{R}^{n}\right)$ tais que

$$
\langle x-y, F(x)-F(y)\rangle \leq 0, \quad \forall(x, y) \in \mathbb{R}^{n} \times \mathbb{R}^{n} .
$$

Então existe $u \in \mathscr{C}^{\infty}\left(\left[t_{0},+\infty\left[; \mathbb{R}^{n}\right)\right.\right.$ tal que

1. $\frac{d u}{d t}(t)=F(u(t)), \quad \forall t \geq t_{0} \quad e \quad u\left(t_{0}\right)=x_{0}$;

2. $\left|u(t)-x_{0}\right| \leq\left(t-t_{0}\right)\left|F\left(x_{0}\right)\right|, \quad \forall t \geq t_{0}$.

Demonstração: Seja $f:] a,+\infty\left[\times \mathbb{R}^{n} \longrightarrow \mathbb{R}^{n}\right.$ definida por

$$
f(t, x)=F(x)
$$

Então $f$ satisfaz a hipótese da Proposição 3.3.5. Logo, existe $u \in \mathscr{C}^{\infty}\left(\left[t_{0},+\infty\left[; \mathbb{R}^{n}\right)\right.\right.$ tal que

$$
\begin{gathered}
\frac{d u}{d t}(t)=f(t, u(t))=F(u(t)), \quad \forall t \geq t_{0} ; u\left(t_{0}\right)=x_{0} ; \\
\left|u(t)-x_{0}\right| \leq \int_{t_{0}}^{t}\left|f\left(s, x_{0}\right)\right| d s=\int_{t_{0}}^{t}\left|F\left(x_{0}\right)\right| d s=\left(t-t_{0}\right)\left|F\left(x_{0}\right)\right|, \quad \forall t \geq t_{0},
\end{gathered}
$$

e assim concluímos a prova.

Iniciaremos a seguir o estudo do problema (3.81), que é o objetivo desta seção. Para isso apresentaremos resultados a nível de representantes em $\mathcal{E}_{\tau}\left[\left[t_{0},+\infty\left[; \mathbb{R}^{n}\right]\right.\right.$ e em $\mathcal{E}_{M}\left[\left[t_{0},+\infty\left[; \mathbb{R}^{n}\right]\right.\right.$.

Proposição 3.3.7 Sejam $t_{0} \in \mathbb{R},\left(u_{\varepsilon}\right)_{\varepsilon} \in\left(\mathscr{C}^{\infty}\left(\left[t_{0},+\infty\left[; \mathbb{R}^{n}\right)\right)^{\mathbf{I}} e\left(F_{\varepsilon}\right)_{\varepsilon} \in \mathcal{E}_{\tau}\left[\mathbb{R}^{n} ; \mathbb{R}^{n}\right]\right.\right.$, tais que

$$
\text { (i) } \frac{d u_{\varepsilon}}{d t}(t)=F_{\varepsilon}\left(u_{\varepsilon}(t)\right), \quad \forall(\varepsilon, t) \in \mathbf{I} \times\left[t_{0},+\infty[\text {; }\right.
$$


(ii) $\forall t_{1}>t_{0}, \exists N_{1} \in \mathbb{N}, \exists c_{1}>0$ e $\exists \eta_{1} \in \mathbf{I}$ satisfazendo

$$
\left|u_{\varepsilon}(t)\right| \leq c_{1} \varepsilon^{-N_{1}}, \quad \forall(\varepsilon, t) \in \mathbf{I}_{\eta_{1}} \times\left[t_{0}, t_{1}\right] .
$$

$\operatorname{Então}\left(u_{\varepsilon}\right)_{\varepsilon} \in \mathcal{E}_{M}\left[\left[t_{0},+\infty\left[; \mathbb{R}^{n}\right]\right.\right.$.

Demonstração: Seja $t_{1}>t_{0}$. Provaremos, pelo Pricípio da Indução Finita, que, para todo $p \in \mathbb{N}$, existem $N \in \mathbb{N}, c>0$ e $\eta \in \mathbf{I}$ tais que

$$
\left|\frac{d^{p} u_{\varepsilon}}{d t^{p}}(t)\right| \leq c \varepsilon^{-N}, \quad \forall(\varepsilon, t) \in \mathbf{I}_{\eta} \times\left[t_{0}, t_{1}\right],
$$

e como $t_{1}>t_{0}$ é arbitrário segue que $\left(u_{\varepsilon}\right)_{\varepsilon} \in \mathcal{E}_{M}\left[\left[t_{0},+\infty\left[; \mathbb{R}^{n}\right]\right.\right.$.

Seja $p \in \mathbb{N}$.

De (ii), existem $N_{1} \in \mathbb{N}, \quad c_{1}>0 \quad$ e $\quad \eta_{1} \in \mathbf{I}$ satisfazendo (3.97). Como $\left(F_{\varepsilon}\right)_{\varepsilon} \in \mathcal{E}_{\tau}\left[\mathbb{R}^{n} ; \mathbb{R}^{n}\right]$, existem $N_{2} \in \mathbb{N}$ com $N_{2}>N_{1}, c_{2}>c_{1}$ e $\eta_{2} \in \mathbf{I}$ com $\eta_{2}<\eta_{1}$ tais que

$$
\left|\partial^{\beta} F_{\varepsilon}(y)\right| \leq c_{2}(1+|y|)^{N_{2}} \varepsilon^{-N_{2}}, \quad \forall(\varepsilon, y) \in \mathbf{I}_{\eta_{2}} \times \mathbb{R}^{n} \text { e } \forall \beta \in \mathbb{N}^{n} \operatorname{com}|\beta| \leq p .
$$

Note que, de (3.97) e (3.99) temos, para $(\varepsilon, t) \in \mathbf{I}_{\eta_{2}} \times\left[t_{0}, t_{1}\right]$ e $\beta \in \mathbb{N}^{n} \operatorname{com}|\beta| \leq p$,

$$
\begin{aligned}
\left|\partial^{\beta} F_{\varepsilon}\left(u_{\varepsilon}(t)\right)\right| & \leq c_{2}\left(1+\left|u_{\varepsilon}(t)\right|\right)^{N_{2}} \varepsilon^{-N_{2}} \\
& \leq c_{2}\left(1+c_{1} \varepsilon^{-N_{1}}\right)^{N_{2}} \varepsilon^{-N_{2}} \\
& \leq c_{2}\left(\varepsilon^{-N_{1}}+c_{1} \varepsilon^{-N_{1}}\right)^{N_{2}} \varepsilon^{-N_{2}} \\
& \leq c_{2}\left(1+c_{1}\right)^{N_{2}} \varepsilon^{-N_{2}{ }^{2}} \varepsilon^{-N_{2}},
\end{aligned}
$$

e assim, para todo $\beta \in \mathbb{N}^{n} \operatorname{com}|\beta| \leq p$, temos

$$
\left|\partial^{\beta} F_{\varepsilon}\left(u_{\varepsilon}(t)\right)\right| \leq c_{2}\left(1+c_{2}\right)^{N_{2}} \varepsilon^{-\left(N_{2}^{2}+N_{2}\right)}, \forall(\varepsilon, t) \in \mathbf{I}_{\eta_{2}} \times\left[t_{0}, t_{1}\right] .
$$

Observe que, por (3.97), (i) e (3.100) com $|\beta|=0$, temos que (3.98) é verdadeira para $p=0$ ou $p=1$.

Suponhamos $p \geq 2$ e que (3.98) seja verdadeira para todo $s \in \mathbb{N}$ com $s \leq p-1$. Logo, existem $N_{3} \in \mathbb{N}, c_{3}>0$ e $\eta_{3} \in \mathbf{I}$ com $\eta_{3}<\eta_{2}$ tais que

$$
\left|\frac{d^{s} u_{\varepsilon}}{d t^{s}}(t)\right| \leq c_{3} \varepsilon^{-N_{3}}, \quad \forall(\varepsilon, t) \in \mathbf{I}_{\eta_{3}} \times\left[t_{0}, t_{1}\right] \quad \text { e } \forall s \in \mathbb{N} \operatorname{com} s \leq p-1 .
$$


Admita que $\left(u_{\varepsilon}\right)_{\varepsilon}:=\left(\left(u_{1_{\varepsilon}}, \cdots, u_{n_{\varepsilon}}\right)\right)_{\varepsilon} \quad$ e $\quad\left(F_{\varepsilon}\right)_{\varepsilon}:=\left(\left(F_{1_{\varepsilon}}, \cdots, F_{n_{\varepsilon}}\right)\right)_{\varepsilon} . \quad$ Fixado $(\varepsilon, t) \in \mathbf{I}_{\eta_{3}} \times\left[t_{0}, t_{1}\right]$, considere os conjuntos

$$
\begin{gathered}
A:=\left\{\frac{d^{s} u_{i_{\varepsilon}}}{d t^{s}}(t), \mid 0 \leq s \leq p-1 \quad \text { e } \quad 1 \leq i \leq n\right\} \\
B:=\left\{\partial^{\beta} F_{i_{\varepsilon}}\left(u_{\varepsilon}(t)\right)|| \beta \mid \leq p-1 \quad \text { e } \quad 1 \leq i \leq n\right\},
\end{gathered}
$$

e note que, por $(i)$, cada coordenada de $\frac{d^{p} u_{\varepsilon}}{d t^{p}}(t)$ é soma e produto de elementos de $A \cup B$. Assim, de (3.100) e (3.101), temos que existem $N_{4} \in \mathbb{N}$ e $c_{4}>0$ tais que

$$
\left|\frac{d^{p} u_{\varepsilon}}{d t^{p}}(t)\right| \leq c_{4} \varepsilon^{-N_{4}}, \quad \forall(\varepsilon, t) \in \mathbf{I}_{\eta_{3}} \times\left[t_{0}, t_{1}\right] \quad \text { e } \quad 1 \leq i \leq n .
$$

Portanto (3.98) é verdadeira para $p$.

A Proposição 3.3.8, que enunciaremos a seguir, possui, em relação à Proposição 3.3.7, uma condição temperada na hipótese $(i i)$. Como conseqüência desse fato, pode-se garantir que o elemento $\left(u_{\varepsilon}\right)_{\varepsilon}$, além de pertencer a $\mathcal{E}_{M}\left[\left[t_{0},+\infty\left[; \mathbb{R}^{n}\right]\right.\right.$, pertence a $\mathcal{E}_{\tau}\left[\left[t_{0},+\infty\left[; \mathbb{R}^{n}\right]\right.\right.$.

Proposição 3.3.8 Sejam $t_{0} \in \mathbb{R},\left(u_{\varepsilon}\right)_{\varepsilon} \in\left(\mathscr{C}^{\infty}\left(\left[t_{0},+\infty\left[; \mathbb{R}^{n}\right)\right)^{\mathbf{I}}\right.\right.$ e $\left(F_{\varepsilon}\right)_{\varepsilon} \in \mathcal{E}_{\tau}\left[\mathbb{R}^{n} ; \mathbb{R}^{n}\right]$, tais que

(i) $\frac{d u_{\varepsilon}}{d t}(t)=F_{\varepsilon}\left(u_{\varepsilon}(t)\right), \quad \forall(\varepsilon, t) \in \mathbf{I} \times\left[t_{0},+\infty[;\right.$

(ii) $\exists N_{1} \in \mathbb{N}, \exists c_{1}>0$ e $\exists \eta_{1} \in \mathbf{I}$ satisfazendo

$$
\left|u_{\varepsilon}(t)\right| \leq c_{1}(1+|t|)^{N_{1}} \varepsilon^{-N_{1}}, \quad \forall(\varepsilon, t) \in \mathbf{I}_{\eta_{1}} \times\left[t_{0},+\infty[\right.
$$

$\operatorname{Enta\tilde {O}}\left(u_{\varepsilon}\right)_{\varepsilon} \in \mathcal{E}_{\tau}\left[\left[t_{0},+\infty\left[; \mathbb{R}^{n}\right]\right.\right.$.

Demonstração: Provaremos, pelo Pricípio da Indução Finita, que, para todo $p \in \mathbb{N}$, existem $N \in \mathbb{N}, c>0$ e $\eta \in \mathbf{I}$ tais que

$$
\left|\frac{d^{p} u_{\varepsilon}}{d t^{p}}(t)\right| \leq c(1+|t|)^{N} \varepsilon^{-N}, \quad \forall(\varepsilon, t) \in \mathbf{I}_{\eta} \times\left[t_{0},+\infty[.\right.
$$


Da hipótese (ii), existem $N_{1} \in \mathbb{N}, c_{1}>0$ e $\eta_{1} \in \mathbf{I}$ satisfazendo (3.102). Como $\left(F_{\varepsilon}\right)_{\varepsilon} \in \mathcal{E}_{\tau}\left[\mathbb{R}^{n} ; \mathbb{R}^{n}\right]$, existem $N_{2}>N_{1}, c_{2}>c_{1}$ e $\eta_{2} \in \mathbf{I}$ com $\eta_{2}<\eta_{1}$ tais que

$$
\left|\partial^{\beta} F_{\varepsilon}(y)\right| \leq c_{2}(1+|y|)^{N_{2}} \varepsilon^{-N_{2}}, \quad \forall(\varepsilon, y) \in \mathbf{I}_{\eta_{2}} \times \mathbb{R}^{n} \quad \text { e } \quad \forall \beta \in \mathbb{N}^{n} \operatorname{com}|\beta| \leq p .
$$

De (3.102) e (3.103) temos, para $(\varepsilon, t) \in \mathbf{I}_{\eta_{2}} \times\left[t_{0},+\infty\left[\right.\right.$ e $\beta \in \mathbb{N}^{n}$ com $|\beta| \leq p$, que

$$
\begin{aligned}
\left|\partial^{\beta} F_{\varepsilon}\left(t, u_{\varepsilon}(t)\right)\right| & \leq c_{2}\left(1+\left|u_{\varepsilon}(t)\right|\right)^{N_{2}} \varepsilon^{-N_{2}} \\
& \leq c_{2}\left(1+c_{1}(1+|t|)^{N_{1}} \varepsilon^{-N_{1}}\right)^{N_{2}} \varepsilon^{-N_{2}} \\
& \leq c_{2}\left(\varepsilon^{-N_{2}}+c_{2}(1+|t|)^{N_{2}} \varepsilon^{-N_{2}}\right)^{N_{2}} \varepsilon^{-N_{2}} \\
& \leq c_{2}\left(\left(1+c_{2}(1+|t|)^{N_{2}}\right) \varepsilon^{-N_{2}}\right)^{N_{2}} \varepsilon^{-N_{2}} \\
& \leq c_{2}\left((1+|t|)^{N_{2}}+c_{2}(1+|t|)^{N_{2}}\right)^{N_{2}} \varepsilon^{-\left(N_{2}{ }^{2}+N_{2}\right)} \\
& \leq c_{2}\left(1+c_{2}\right)^{N_{2}}(1+|t|)^{\left(N_{2}{ }^{2}+N_{2}\right)} \varepsilon^{-\left(N_{2}{ }^{2}+N_{2}\right)} .
\end{aligned}
$$

Portanto, para $c_{3}:=c_{2}\left(1+c_{2}\right)^{N_{2}}$ e $N_{3}:=N_{2}^{2}+N_{2}$ tem-se, para todo $\beta \in \mathbb{N}^{n}$ com $|\beta| \leq p$, que

$$
\left|\partial^{\beta} F_{\varepsilon}\left(t, u_{\varepsilon}(t)\right)\right| \leq c_{3}(1+|t|)^{N_{3}} \varepsilon^{-N_{3}}, \quad \forall(\varepsilon, t) \in \mathbf{I}_{\eta_{2}} \times\left[t_{0},+\infty[.\right.
$$

Note que, por (3.102), (i) e (3.105) com $|\beta|=0$, temos que a afirmação (3.103) é verdadeira para $p=0$ ou $p=1$.

Suponhamos $p \geq 2$ e que (3.103) seja verdadeira para todo $s \in \mathbb{N}$ com $s \leq p-1$. Então, existem $N_{4} \in \mathbb{N}, c_{4}>0$ e $\eta_{3} \in \mathbf{I} \operatorname{com} \eta_{3}<\eta_{2}$ tais que $\left|\frac{d^{s} u_{\varepsilon}}{d t^{s}}(t)\right| \leq c_{4}(1+|t|)^{N_{4}} \varepsilon^{-N_{4}}, \quad \forall(\varepsilon, t) \in \mathbf{I}_{\eta_{3}} \times\left[t_{0},+\infty[\right.$ e $\forall s \in \mathbb{N}$ com $s \leq p-1$.

Considere $\quad\left(u_{\varepsilon}\right)_{\varepsilon}:=\left(\left(u_{1_{\varepsilon}}, \cdots, u_{n_{\varepsilon}}\right)\right)_{\varepsilon} \quad$ e $\quad\left(F_{\varepsilon}\right)_{\varepsilon}:=\left(\left(F_{1_{\varepsilon}}, \cdots, F_{n_{\varepsilon}}\right)\right)_{\varepsilon}$. Fixado $(\varepsilon, t) \in \mathbf{I}_{\eta_{3}} \times\left[t_{0}, t_{1}\right]$, sejam

$$
\begin{gathered}
A:=\left\{\frac{d^{s} u_{i_{\varepsilon}}}{d t^{s}}(t) \mid 0 \leq s \leq p-1 \text { e } 1 \leq i \leq n\right\} \\
B:=\left\{\partial^{\beta} F_{i_{\varepsilon}}\left(u_{\varepsilon}(t)\right)|| \beta \mid \leq p-1 \text { e } 1 \leq i \leq n\right\} .
\end{gathered}
$$


Então, por $(i)$, cada coordenada de $\frac{d^{p} u_{\varepsilon}}{d t^{p}}(t)$ é soma e produto de elementos de $A \cup B$. Assim, de (3.105) e (3.106), temos que existem $N_{5} \in \mathbb{N}$ e $c_{5}>0$ tais que

$$
\left|\frac{d^{p} u_{\varepsilon}}{d t^{p}}(t)\right| \leq c_{5}(1+|t|)^{N_{5}} \varepsilon^{-N_{5}}, \quad \forall(\varepsilon, t) \in \mathbf{I}_{\eta_{3}} \times\left[t_{0},+\infty[\quad \text { e } \quad 1 \leq i \leq n .\right.
$$

Portanto (3.103) é verdadeira para $p$.

Finalizamos esta seção com um teorema de existência e unicidade de solução para o problema (3.81), onde $F \in \mathcal{G}_{\tau}\left(\mathbb{R}^{n} ; \mathbb{R}^{n}\right)$ é dissipativa em $\mathcal{G}_{\tau}\left(\mathbb{R}^{n} ; \mathbb{R}^{n}\right)$ (Definição 3.3.1).

Teorema 3.3.9 Seja $F \in \mathcal{G}_{\tau}\left(\mathbb{R}^{n} ; \mathbb{R}^{n}\right)$ disssipativa em $\mathcal{G}_{\tau}\left(\mathbb{R}^{n} ; \mathbb{R}^{n}\right)$. Então o problema (3.81) tem uma única solução em $\mathcal{G}\left(\left[t_{0},+\infty\left[; \mathbb{R}^{n}\right)\right.\right.$ e uma única solução em $\mathcal{G}_{\tau}\left(\left[t_{0},+\infty\left[; \mathbb{R}^{n}\right)\right.\right.$.

Demonstração: Sejam $\left(x_{0}\right)_{\varepsilon}$ um representante de $x_{0} \in \overline{\mathbb{R}}^{n}, t_{0} \in \mathbb{R}$. De $F$ ser dissipativa em $\mathcal{G}_{\tau}\left(\mathbb{R}^{n} ; \mathbb{R}^{n}\right)$, existe $\left(F_{\varepsilon}\right)_{\varepsilon}$ um representante de $F$ satisfazendo (3.82). Portanto, pelo Teorema 3.3.6, para todo $\varepsilon \in \mathbf{I}$, existe $u_{\varepsilon} \in \mathscr{C}^{\infty}\left(\left[t_{0},+\infty\left[; \mathbb{R}^{n}\right)\right.\right.$ tal que

$$
\begin{gathered}
\frac{d u_{\varepsilon}}{d t}(t)=F_{\varepsilon}\left(u_{\varepsilon}(t)\right), \quad \forall t \geq t_{0} ; \\
u_{\varepsilon}\left(t_{0}\right)=x_{0_{\varepsilon}} ; \\
\left|u_{\varepsilon}(t)-x_{0_{\varepsilon}}\right| \leq\left(t-t_{0}\right)\left|F_{\varepsilon}\left(x_{0_{\varepsilon}}\right)\right|, \quad \forall t \geq t_{0} .
\end{gathered}
$$

Provaremos, a seguir, que $\left(u_{\varepsilon}\right)_{\varepsilon} \in \mathcal{E}_{\tau}\left[\left[t_{0},+\infty\left[; \mathbb{R}^{n}\right]\right.\right.$, e assim, pela Proposição 1.5.11 (1.), teremos que $\left(u_{\varepsilon}\right)_{\varepsilon}+\mathcal{N}_{\tau}\left[\left[t_{0},+\infty\left[; \mathbb{R}^{n}\right]\right.\right.$ e $\left(u_{\varepsilon}\right)_{\varepsilon}+\mathcal{N}\left[\left[t_{0},+\infty\left[; \mathbb{R}^{n}\right]\right.\right.$ são soluções do problema $(3.81)$ em $\mathcal{G}_{\tau}\left(\left[t_{0},+\infty\left[; \mathbb{R}^{n}\right)\right.\right.$ e em $\mathcal{G}\left(\left[t_{0},+\infty\left[; \mathbb{R}^{n}\right)\right.\right.$, respectivamente.

De $\left(x_{0}\right)_{\varepsilon} \in\left(\mathcal{E}_{M}(\mathbb{R})\right)^{n}$ e $\left(F_{\varepsilon}\right)_{\varepsilon} \in \mathcal{E}_{\tau}\left[\mathbb{R}^{n} ; \mathbb{R}^{n}\right]$, existem $N_{1} \in \mathbb{N}, c_{1}>1$ e $\eta \in \mathbf{I}$, tais que

$$
\begin{gathered}
\left|x_{0_{\varepsilon}}\right| \leq c_{1} \varepsilon^{-N_{1}}, \quad \forall \in \mathbf{I}_{\eta} ; \\
\left|F_{\varepsilon}(x)\right| \leq c_{1}(1+|x|)^{N_{1}} \varepsilon^{-N_{1}}, \quad \forall(\varepsilon, x) \in \mathbf{I}_{\eta} \times \mathbb{R}^{n} .
\end{gathered}
$$


Seja $T:=\max \left\{\left|t_{0}\right|, 1\right\}$. De (3.109), (3.110) e (3.111) tem-se

$$
\begin{aligned}
\left|u_{\varepsilon}(t)\right| & \leq\left|u_{\varepsilon}(t)-x_{0_{\varepsilon}}\right|+\left|x_{0_{\varepsilon}}\right| \\
& \leq\left(t-t_{0}\right)\left|F_{\varepsilon}\left(x_{0_{\varepsilon}}\right)\right|+\left|x_{0_{\varepsilon}}\right| \\
& \leq\left(t-t_{0}\right) c_{1}\left(1+\left|x_{0_{\varepsilon}}\right|\right)^{N_{1}} \varepsilon^{-N_{1}}+c_{1} \varepsilon^{-N_{1}} \\
& \leq\left(|t|+\left|t_{0}\right|\right) c_{1}\left(1+c_{1} \varepsilon^{-N_{1}}\right)^{N_{1}} \varepsilon^{-N_{1}}+c_{1} \varepsilon^{-N_{1}} \\
& \leq(T|t|+T) c_{1}\left(\varepsilon^{-N_{1}}+c_{1} \varepsilon^{-N_{1}}\right)^{N_{1}} \varepsilon^{-N_{1}}+c_{1} \varepsilon^{-N_{1}} \\
& \leq T(1+|t|) c_{1}\left(1+c_{1}\right)^{N_{1}} \varepsilon^{-\left(N_{1}^{2}+N_{1}\right)}+c_{1} \varepsilon^{-N_{1}} \\
& \leq c_{1} T\left(1+c_{1}\right)^{N_{1}}(1+|t|) \varepsilon^{-\left(N_{1}^{2}+N_{1}\right)}+c_{1} T(1+|t|) \varepsilon^{-N_{1}} \\
& \leq 2 c_{1} T\left(1+c_{1}\right)^{N_{1}}(1+|t|) \varepsilon^{-\left(N_{1}^{2}+N_{1}\right)},
\end{aligned}
$$

para todo $(\varepsilon, t) \in \mathbf{I}_{\eta} \times\left[t_{0},+\infty[\right.$.

Sejam $N:=1+N_{1}^{2}+N_{1}$ e $c:=2 c_{1} T\left(1+c_{1}\right)^{N_{1}}$. Então

$$
\left|u_{\varepsilon}(t)\right| \leq c(1+|t|)^{N} \varepsilon^{-N}, \quad \forall(\varepsilon, t) \in \mathbf{I}_{\eta} \times\left[t_{0},+\infty[.\right.
$$

Portanto, pela Proposição 3.3.8, $\left(u_{\varepsilon}\right)_{\varepsilon} \in \mathcal{E}_{\tau}\left[\left[t_{0},+\infty\left[; \mathbb{R}^{n}\right]\right.\right.$.

Para concluirmos a prova falta verificarmos que há uma única solução para o problema (3.81).

Iniciamos provando a unicidade em $\mathcal{G}\left(\left[t_{0},+\infty\left[; \mathbb{R}^{n}\right)\right.\right.$.

$\operatorname{Sejam~}\left(v_{\varepsilon}\right)_{\varepsilon} \in \mathcal{E}_{M}\left[\left[t_{0},+\infty\left[; \mathbb{R}^{n}\right]\right.\right.$ e $v:=\left(v_{\varepsilon}\right)_{\varepsilon}+\mathcal{N}\left[\left[t_{0},+\infty\left[; \mathbb{R}^{n}\right]\right.\right.$ uma outra solução do problema $(3.81)$ em $\mathcal{G}\left(\left[t_{0},+\infty\left[; \mathbb{R}^{n}\right)\right.\right.$. Então existem $\left(n_{\varepsilon}\right)_{\varepsilon} \in \mathcal{N}\left[\left[t_{0},+\infty\left[; \mathbb{R}^{n}\right]\right.\right.$ e $\left(m_{\varepsilon}\right)_{\varepsilon} \in(\mathcal{N}(\mathbb{R}))^{n}$ tais que

$$
\begin{gathered}
\frac{d v_{\varepsilon}}{d t}(t)=F_{\varepsilon}\left(v_{\varepsilon}(t)\right)+n_{\varepsilon}(t), \quad \forall(\varepsilon, t) \in \mathbf{I} \times\left[t_{0},+\infty[;\right. \\
v_{\varepsilon}\left(t_{0}\right)=x_{0_{\varepsilon}}+m_{\varepsilon}, \quad \forall \varepsilon \in \mathbf{I} .
\end{gathered}
$$

Provaremos que $\left(u_{\varepsilon}-v_{\varepsilon}\right)_{\varepsilon} \in \mathcal{N}\left[\left[t_{0},+\infty\left[; \mathbb{R}^{n}\right]\right.\right.$. Antes porém, notemos que, para todo $(\varepsilon, t) \in \mathbf{I} \times\left[t_{0},+\infty[\right.$, tem-se, por (3.82), (3.107) e (3.112), que

$$
\frac{1}{2} \frac{d}{d t}\left|u_{\varepsilon}(t)-v_{\varepsilon}(t)\right|^{2}=\left\langle u_{\varepsilon}(t)-v_{\varepsilon}(t), \frac{d}{d t}\left(u_{\varepsilon}(t)-v_{\varepsilon}(t)\right)\right\rangle
$$




$$
\begin{aligned}
& =\left\langle u_{\varepsilon}(t)-v_{\varepsilon}(t), F_{\varepsilon}\left(u_{\varepsilon}(t)\right)-F_{\varepsilon}\left(v_{\varepsilon}(t)\right)-n_{\varepsilon}(t)\right\rangle \\
& =\left\langle u_{\varepsilon}(t)-v_{\varepsilon}(t), F_{\varepsilon}\left(u_{\varepsilon}(t)\right)-F_{\varepsilon}\left(v_{\varepsilon}(t)\right)\right\rangle+\left\langle u_{\varepsilon}(t)-v_{\varepsilon}(t),-n_{\varepsilon}(t)\right\rangle \\
& \leq\left\langle u_{\varepsilon}(t)-v_{\varepsilon}(t),-n_{\varepsilon}(t)\right\rangle \\
& \leq\left|n_{\varepsilon}(t)\right|\left|u_{\varepsilon}(t)-v_{\varepsilon}(t)\right|
\end{aligned}
$$

ou seja

$$
\frac{d}{d t}\left(\left|u_{\varepsilon}(t)-v_{\varepsilon}(t)\right|^{2}\right) \leq 2\left|n_{\varepsilon}(t)\right|\left|u_{\varepsilon}(t)-v_{\varepsilon}(t)\right|, \quad \forall(\varepsilon, t) \in \mathbf{I} \times\left[t_{0},+\infty[.\right.
$$

\section{Portanto}

$\left|u_{\varepsilon}(t)-v_{\varepsilon}(t)\right|^{2}-\left|u_{\varepsilon}\left(t_{0}\right)-v_{\varepsilon}\left(t_{0}\right)\right|^{2} \leq \int_{t_{0}}^{t} 2\left|n_{\varepsilon}(s)\right|\left|u_{\varepsilon}(s)-v_{\varepsilon}(s)\right| d s, \quad \forall(\varepsilon, t) \in \mathbf{I} \times\left[t_{0},+\infty[\right.$,

e assim

$$
\left|u_{\varepsilon}(t)-v_{\varepsilon}(t)\right|^{2} \leq\left|m_{\varepsilon}\right|^{2}+\int_{t_{0}}^{t} 2\left|n_{\varepsilon}(s)\right|\left|u_{\varepsilon}(s)-v_{\varepsilon}(s)\right| d s, \quad \forall(\varepsilon, t) \in I \times\left[t_{0},+\infty[.\right.
$$

Sejam $t_{1}>t_{0}$ e $q \in \mathbb{N}$. De $\left(u_{\varepsilon}\right)_{\varepsilon},\left(v_{\varepsilon}\right)_{\varepsilon} \in \mathcal{E}_{M}\left[\left[t_{0},+\infty\left[; \mathbb{R}^{n}\right]\right.\right.$, existem $c_{1}>0, N_{1} \in \mathbb{N}$ e $\eta_{1} \in \mathbf{I}$ tais que

$$
\begin{aligned}
& \left|u_{\varepsilon}(t)\right| \leq c_{1} \varepsilon^{-N_{1}}, \quad \forall(\varepsilon, t) \in \mathbf{I}_{\eta_{1}} \times\left[t_{0}, t_{1}\right] \\
& \left|v_{\varepsilon}(t)\right| \leq c_{1} \varepsilon^{-N_{1}}, \quad \forall(\varepsilon, t) \in \mathbf{I}_{\eta_{1}} \times\left[t_{0}, t_{1}\right] .
\end{aligned}
$$

Logo

$$
\left|u_{\varepsilon}(t)-v_{\varepsilon}(t)\right| \leq\left|u_{\varepsilon}(t)\right|+\left|v_{\varepsilon}(t)\right| \leq 2 c_{1} \varepsilon^{-N_{1}}, \quad \forall(\varepsilon, t) \in \mathbf{I}_{\eta_{1}} \times\left[t_{0}, t_{1}\right]
$$

Como $\left(m_{\varepsilon}\right)_{\varepsilon} \in(\mathcal{N}(\mathbb{R}))^{n}$ e $\left(n_{\varepsilon}\right)_{\varepsilon} \in \mathcal{N}\left[\left[t_{0},+\infty\left[; \mathbb{R}^{n}\right]\right.\right.$, existem $c_{2}>c_{1}$ e $\eta_{2} \in \mathbf{I}$ com $\eta_{2}<\eta_{1}$ tais que

$$
\begin{gathered}
\left|m_{\varepsilon}\right| \leq c_{2} \varepsilon^{q}, \quad \forall \varepsilon \in \mathbf{I}_{\eta_{2}} ; \\
\left|n_{\varepsilon}(t)\right| \leq c_{2} \varepsilon^{N_{1}+2 q}, \quad \forall(\varepsilon, t) \in \mathbf{I}_{\eta_{1}} \times\left[t_{0}, t_{1}\right] .
\end{gathered}
$$

De (3.114), (3.115), (3.116) e (3.117) temos que

$$
\begin{aligned}
\left|u_{\varepsilon}(t)-v_{\varepsilon}(t)\right|^{2} & \leq c_{2}^{2} \varepsilon^{2 q}+\int_{t_{0}}^{t} 2 c_{2} \varepsilon^{N_{1}+2 q} 2 c_{1} \varepsilon^{-N_{1}} d s \\
& \leq c_{2}^{2} \varepsilon^{2 q}+4 c_{2}^{2} \varepsilon^{2 q}\left|t-t_{0}\right| \\
& \leq\left(c_{2}^{2}+4 c_{2}^{2}\left|t-t_{0}\right|\right) \varepsilon^{2 q},
\end{aligned}
$$


para todo $(\varepsilon, t) \in \mathbf{I}_{\eta_{2}} \times\left[t_{0}, t_{1}\right]$. Logo

$$
\left|u_{\varepsilon}(t)-v_{\varepsilon}(t)\right| \leq\left(c_{2}^{2}+4 c_{2}^{2}\left|t_{1}-t_{0}\right|\right)^{1 / 2} \varepsilon^{q}, \quad \forall(\varepsilon, t) \in \mathbf{I}_{\eta_{2}} \times\left[t_{0}, t_{1}\right],
$$

e $\operatorname{assim}\left(u_{\varepsilon}(t)-v_{\varepsilon}(t)\right)_{\varepsilon}$ satisfaz a condição (b) do Teorema de Nulidade em $\mathcal{E}_{M}\left[\left[t_{0},+\infty\left[; \mathbb{R}^{n}\right]\right.\right.$ (Teorema 1.5.5), ou seja, o problema (3.81) tem uma única solução em $\mathcal{G}\left(\left[t_{0},+\infty\left[; \mathbb{R}^{n}\right)\right.\right.$.

Para finalizar a prova, admita que exista $\left(v_{\varepsilon}\right)_{\varepsilon} \in \mathcal{E}_{\tau}\left[\left[t_{0},+\infty\left[; \mathbb{R}^{n}\right]\right.\right.$ tal que $v:=\left(v_{\varepsilon}\right)_{\varepsilon}+\mathcal{N}_{\tau}\left[\left[t_{0},+\infty\left[; \mathbb{R}^{n}\right]\right.\right.$ seja uma outra solução do problema (3.81) em $\mathcal{G}_{\tau}\left(\left[t_{0},+\infty\left[; \mathbb{R}^{n}\right)\right.\right.$. Então existem $\left(n_{\varepsilon}\right)_{\varepsilon} \in \mathcal{N}_{\tau}\left[\left[t_{0},+\infty\left[; \mathbb{R}^{n}\right]\right.\right.$ e $\left(m_{\varepsilon}\right)_{\varepsilon} \in(\mathcal{N}(\mathbb{R}))^{n}$ tais que (3.112) e (3.113) são verdadeiras.

Provaremos que $\left(u_{\varepsilon}-v_{\varepsilon}\right)_{\varepsilon} \in \mathcal{N}_{\tau}\left[\left[t_{0},+\infty\left[; \mathbb{R}^{n}\right]\right.\right.$. Antes porém, note que (3.114) ainda é verdadeira nesse caso.

De $\left(u_{\varepsilon}\right)_{\varepsilon},\left(v_{\varepsilon}\right)_{\varepsilon} \in \mathcal{E}_{\tau}\left[\left[t_{0},+\infty\left[; \mathbb{R}^{n}\right]\right.\right.$, existem $N_{3} \in \mathbb{N}, c_{3}>0$ e $\eta_{3} \in \mathbf{I}$ tais que

$$
\begin{aligned}
& \left|u_{\varepsilon}(t)\right| \leq c_{3}(1+|t|)^{N_{3}} \varepsilon^{-N_{3}}, \quad \forall(\varepsilon, t) \in \mathbf{I}_{\eta_{3}} \times\left[t_{0},+\infty[;\right. \\
& \left|v_{\varepsilon}(t)\right| \leq c_{3}(1+|t|)^{N_{3}} \varepsilon^{-N_{3}}, \quad \forall(\varepsilon, t) \in \mathbf{I}_{\eta_{3}} \times\left[t_{0},+\infty[.\right.
\end{aligned}
$$

Logo

$$
\left|u_{\varepsilon}(t)-v_{\varepsilon}(t)\right| \leq\left|u_{\varepsilon}(t)\right|+\left|v_{\varepsilon}(t)\right| \leq 2 c_{3}(1+|t|)^{N_{3}} \varepsilon^{-N_{3}}, \quad \forall(\varepsilon, t) \in \mathbf{I}_{\eta_{3}} \times\left[t_{0},+\infty[.\right.
$$

De $\left(n_{\varepsilon}\right)_{\varepsilon} \in \mathcal{N}_{\tau}\left[\left[t_{0},+\infty\left[; \mathbb{R}^{n}\right]\right.\right.$, existe $N_{4} \in \mathbb{N}$ com $N_{4}>N_{3}$ tal que, para todo $r \in \mathbb{N}$, existem $c_{r}>c_{3}$ e $\eta_{r} \in \mathbf{I}$ com $\eta_{r}<\eta_{3}$ satisfazendo

$$
\left|n_{\varepsilon}(t)\right| \leq c_{r}(1+|t|)^{N_{4}} \varepsilon^{r}, \quad \forall(\varepsilon, t) \in \mathbf{I}_{\eta_{r}} \times\left[t_{0},+\infty[.\right.
$$

Sejam $N:=2 N_{4}+1$ e $q \in \mathbb{N}$. Para $r:=N_{3}+2 q$ sejam $c_{4}:=c_{r}$ e $\eta_{4}:=\eta_{r}$ como em (3.119).

De $\left(m_{\varepsilon}\right)_{\varepsilon} \in(\mathcal{N}(\mathbb{R}))^{n}$, existem $c_{5}>c_{4}$ e $\eta_{5} \in \mathbf{I}$ com $\eta_{5}<\eta_{4}$ tais que

$$
\left|m_{\varepsilon}\right| \leq c_{5} \varepsilon^{q}, \quad \forall \varepsilon \in \mathbf{I}_{\eta_{5}} .
$$


Seja $T:=\max \left\{1,\left|t_{0}\right|\right\}$. De (3.114), (3.118), (3.119) para $r:=N_{3}+2 q$, e de $(3.120)$ temos que

$$
\begin{aligned}
\left|u_{\varepsilon}(t)-v_{\varepsilon}(t)\right|^{2} & \leq c_{5}^{2} \varepsilon^{2 q}+\int_{t_{0}}^{t} 2 c_{4}(1+|s|)^{N_{4}} \varepsilon^{N_{3}+2 q} 2 c_{3}(1+|s|)^{N_{3}} \varepsilon^{-N_{3}} d s \\
& \leq c_{5}^{2} \varepsilon^{2 q}+\int_{t_{0}}^{t} 4 c_{4}^{2}(1+|s|)^{2 N_{4}} \varepsilon^{2 q} d s \\
& \leq c_{5}^{2} \varepsilon^{2 q}+4 c_{4}^{2}(1+|t|)^{2 N_{4}} \varepsilon^{2 q}\left(|t|+\left|t_{0}\right|\right) \\
& \leq c_{5}^{2} \varepsilon^{2 q}+4 c_{4}^{2}(1+|t|)^{2 N_{4}} \varepsilon^{2 q}(T|t|+T) \\
& \leq c_{5}^{2} \varepsilon^{2 q}+4 c_{4}^{2}(1+|t|)^{2 N_{4}} \varepsilon^{2 q} T(1+|t|) \\
& \leq\left(c_{5}^{2}(1+|t|)^{2 N_{4}+1}+4 T c_{4}^{2}(1+|t|)^{2 N_{4}+1}\right) \varepsilon^{2 q} \\
& \leq(1+4 T) c_{5}^{2}(1+|t|)^{2 N} \varepsilon^{2 q}
\end{aligned}
$$

para todo $(\varepsilon, t) \in \mathbf{I}_{\eta_{5}} \times\left[t_{0}+\infty[\right.$.

Sejam $c:=(1+4 T)^{1 / 2} c_{5}$. Então

$$
\left|u_{\varepsilon}(t)-v_{\varepsilon}(t)\right| \leq c(1+|t|)^{N} \varepsilon^{q}, \quad \forall(\varepsilon, t) \in \mathbf{I}_{\eta_{5}} \times\left[t_{0}+\infty[,\right.
$$

e assim, tem-se que $\left(u_{\varepsilon}-v_{\varepsilon}\right)_{\varepsilon}$ satisfaz a condição $(b)$ do Teorema de Nulidade em $\mathcal{E}_{\tau}\left[\left[t_{0},+\infty\left[; \mathbb{R}^{n}\right]\right.\right.$ (Teorema 1.5.6), ou seja, o problema (3.81) tem uma única solução em $\mathcal{G}_{\tau}\left(\left[t_{0},+\infty\left[; \mathbb{R}^{n}\right)\right.\right.$.

Com esse último resultado encerramos o nosso trabalho sobre a existência e unicidade de solução para equações diferenciais ordinárias no contexto das funçõs generalizadas temperadas de Colombeau. Uma teoria local para funções em $\mathcal{G}\left(\Omega ; \mathbb{R}^{n}\right)$ foi desenvolvida por R. Fernandez em [Fer]. 


\section{Lista de símbolos e notações}

\begin{tabular}{|c|c|}
\hline $\mathbb{R}$ & conjunto dos números reais \\
\hline $\mathbb{C}$ & conjunto dos números complexos \\
\hline$A \backslash B$, onde $A \subset B$ & conjunto $\{a \in A \mid a \notin B\}$ \\
\hline $\mathbb{N}$ & conjunto dos números naturais \\
\hline $\mathbb{N}^{*}$ & $\mathbb{N} \backslash\{0\}$ \\
\hline $\bar{A}$, onde $A \subset \mathbb{R}^{n}$ e $A \neq \mathbb{R}^{n}$ & fecho de $A$ em $\mathbb{R}^{n}$ \\
\hline$B_{r}(x)$, onde $r>0$ e $x \in A$ & bola aberta, de centro $x$ e raio $r$, em $A$ \\
\hline$\langle\rangle$, & produto interno usual de $\mathbb{R}^{n}$ \\
\hline$|x|$, onde $x \in \mathbb{R}^{n}$ & norma de $x$ em $\mathbb{R}^{n}$ \\
\hline$\varepsilon \downarrow 0$ & $\varepsilon \rightarrow 0^{+}$ \\
\hline$\|f\|_{\infty}$ & $\sup \{|f| \mid x \in \Omega\}$, onde $f \in\left(\mathbb{R}^{n}\right)^{\Omega}$ é limitada \\
\hline $\operatorname{supp} \varphi$ & ver início da seção 2.1 \\
\hline $\begin{array}{l}x^{\beta} \text { onde } x \in \mathbb{R}^{n} \text { e } \beta \in \mathbb{N}^{n} \\
\left(\begin{array}{l}\alpha \\
\beta\end{array}\right) \text {, onde } \beta, \alpha \in \mathbb{N}^{n}\end{array}$ & $\begin{array}{l}\left(x_{1}^{\beta_{1}}, \cdots, x_{n}^{\beta^{n}}\right), \text { onde } \beta_{i} \text { é a i-ésima coordenada de } \beta \\
\prod_{i=1}^{n}\left(\begin{array}{c}\alpha_{i} \\
\beta_{i}\end{array}\right) \text {, onde } \beta_{i} \text { e } \alpha_{i} \text { são a i-ésima coordenada de } \beta \text { e }\end{array}$ \\
\hline$|\alpha|$ & $\begin{array}{c}\alpha, \text { respectivamente } \\
\sum_{i=1}^{n} \alpha_{i}, \text { onde } \alpha=\left(\alpha_{1}, \ldots, \alpha_{1}\right) \in \mathbb{N}^{n} \\
\partial^{|\alpha|}\end{array}$ \\
\hline$\partial^{\alpha}$ & $\overline{\partial x_{1}^{\alpha_{1}} \ldots \partial x_{n}^{\alpha_{n}}}$, onde $\alpha=\left(\alpha_{1}, \ldots, \alpha_{1}\right) \in \mathbb{N}^{n}$ \\
\hline$K \subset \subset \Omega$ & $K$ é um subconjunto compacto de $\Omega$ \\
\hline$K$ & interior de $K$ \\
\hline & ver início da seção 1.1 \\
\hline
\end{tabular}




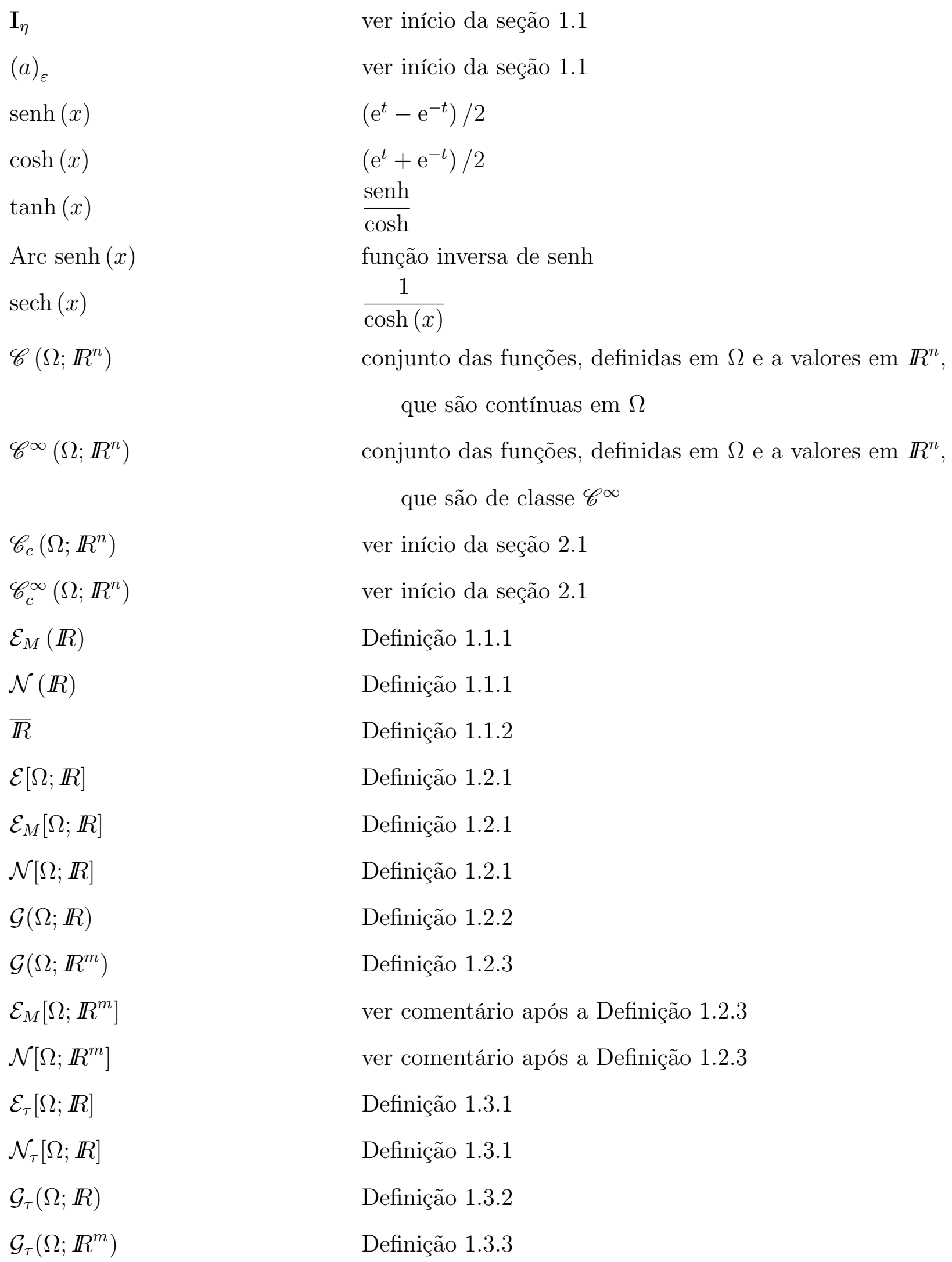

Definição 1.3.3 


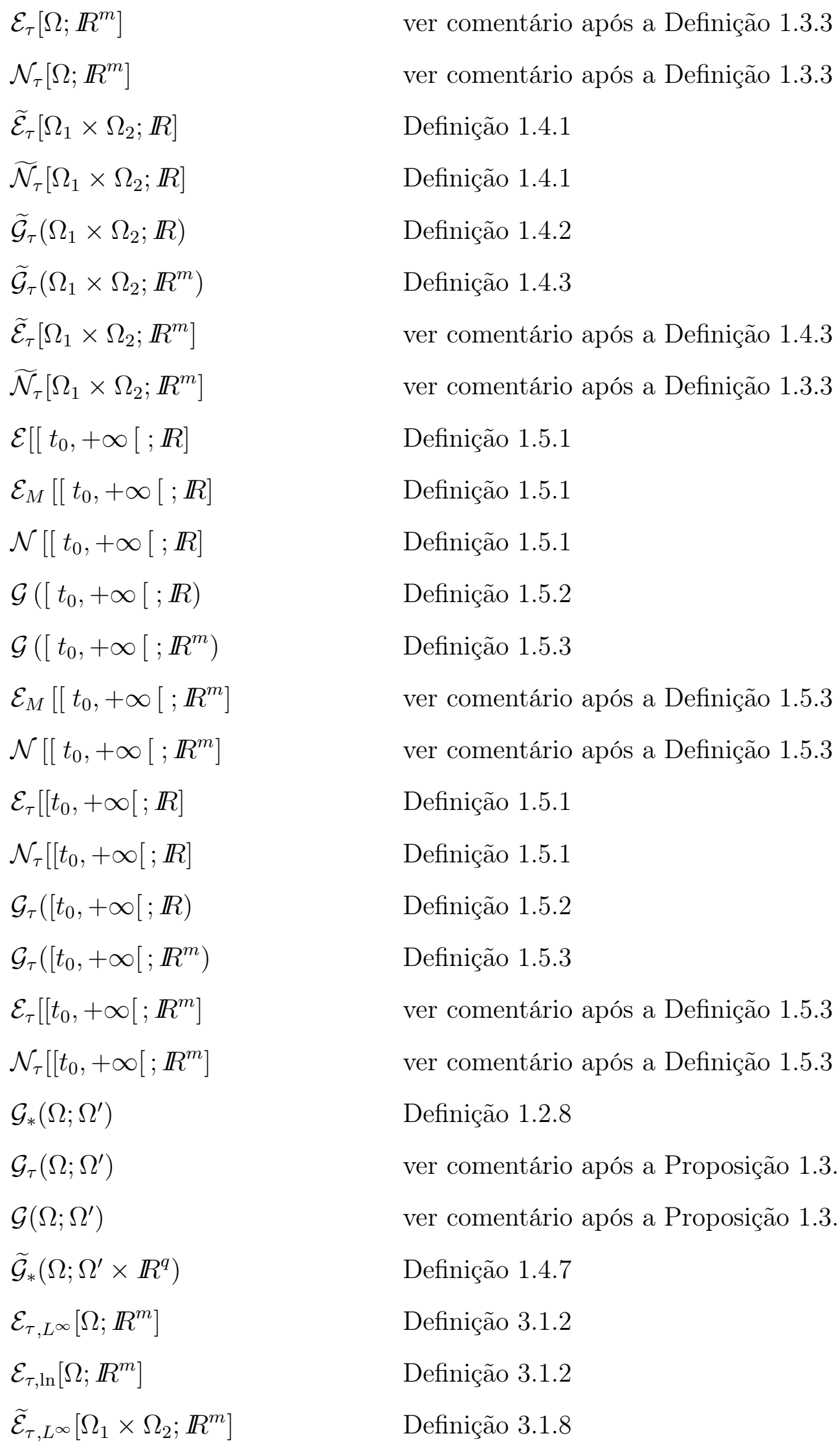

Definição 3.1.8 


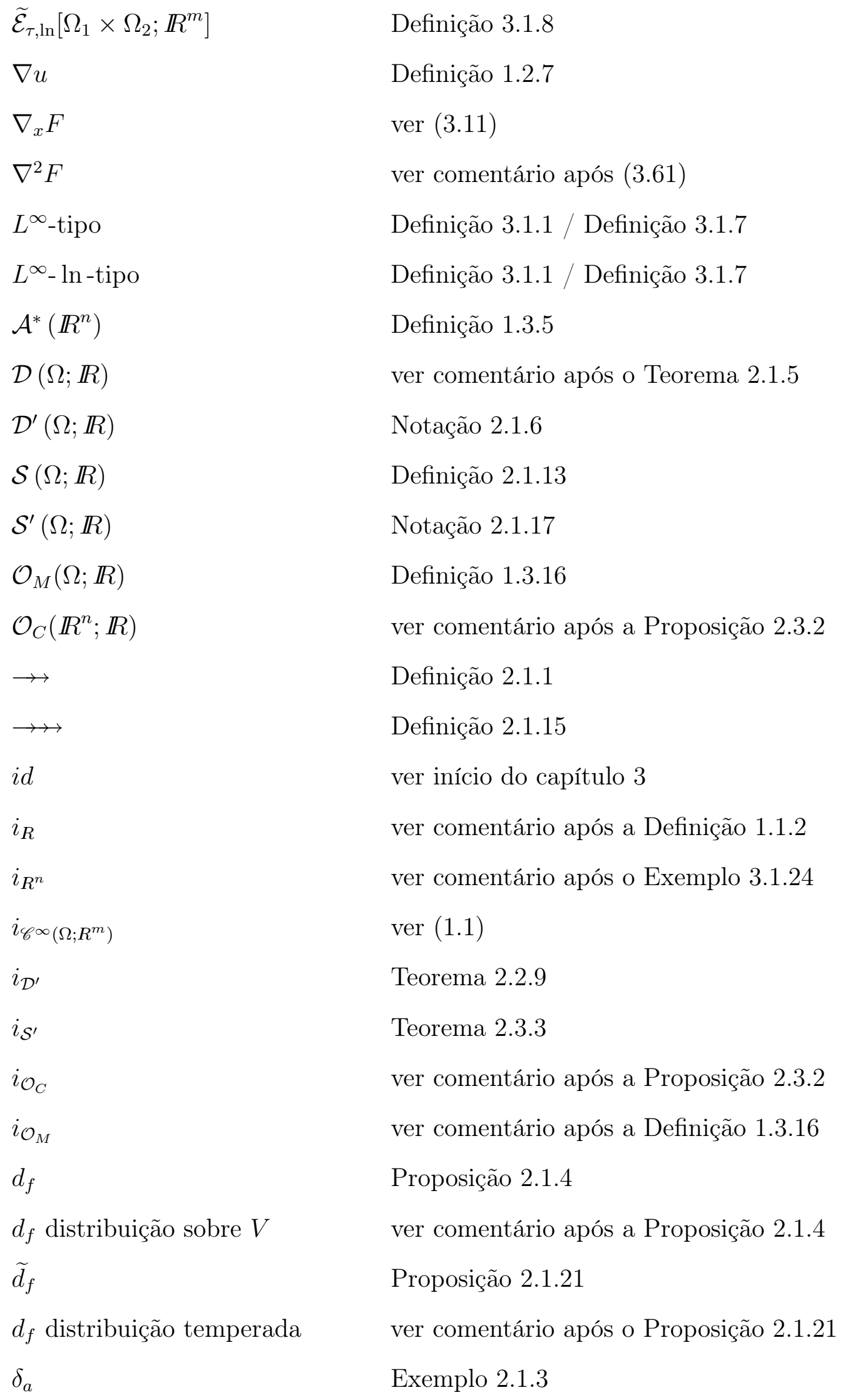


$\delta$

$H_{a}$

$\widetilde{\delta}_{a}$

$\delta_{a}$ distribuição temperada

$C L I$ ver comentário após o Exemplo 2.1.3

Exemplo 2.1.3

Exemplo 2.1.19

ver comentário após o Exemplo 2.1.19

Definição 2.1.20 


\section{Referências Bibliográficas}

[Ara-1] Aragona, J., Colombeau generalized functions on quasi-regular sets, Publ. Math. Debrecen, 68 (2006), 371-399.

[Ara-2] Aragona, J., Introdução à teoria das funções generalizadas de Colombeau, Notas de aula da disciplina MAT829, Universidade de São Paulo, São Paulo, 1989.

[AB] Aragona, J.; Biagioni, H., Intrinsic definition of the Colombeau algebra of generalized functions, Anal. Math., 17 (1991), 75-132.

[AC] Aragona, J.; Colombeau, J. F., The interpolation theorem for holomorphic generalized functions, Ann. Polon. Math., 49 (1988), 151-156.

[AFJ-1] Aragona, J.; Fernandez R.; Juriaans, S. O., A discontinuous Colombeau differential calculus, Monatsh. Math., 144 (2005), 13-29.

[AFJ-2] Aragona, J.; Fernandez R.; Juriaans, S. O., Natural topologies on Colombeau Algebras, preprint.

[AFJ-3] Aragona, J.; Fernandez R.; Juriaans, S. O., The sharp topology on the full Colombeau algebra of generalized functions, Integral Transforms Spec. Funct., 17 (2006), 165-170.

[AJ] Aragona, J.; Juriaans, S. O., Some structural properties of the topological ring of Colombeau's generalized numbers, Comm. Algebra, 29 (2001), 2201-2230. 
[AJOS] Aragona, J.; Juriaans, S. O.; Oliveira O.; Scarpalezos D., Algebraic theory of the topological algebra of Colombeau's generalized functions, preprint.

[AS] Aragona, J.; Soares, M., An existence theorem for an analytic first order PDE in the framework of Colombeau's theory, Monatsh. Math., 134 (2001), 9-17.

[AV] Aragona, J.; Villarreal, F., Colombeau's theory and shock waves in a problem of hydrodynamics, J. Anal. Math., 61 (1993), 113-144.

[Bar] Barros, J. N., An introduction to the theory of distributions, Marcel Dekker, Inc., New York, 1973.

[Bia-1] Biagioni, H. A., A Nonlinear theory of generalized functions, Lecture Notes in mathematics, vol 1421, Springer-Verlag, 1990.

[Bia-2] Biagioni, H. A., Generalized solutions to nonlinear first-order systems, Monatsh. Math., 118(1994), 57-20.

[Chi] Chicone, C; Ordinary differential equations with applications, Texts in Applied Mathematics 34, Springer, New York, 1999.

[Col-1] Colombeau, J. F., Elementary introduction to new generalized functions, NorthHolland, Amsterdam, 1985.

[Col-2] Colombeau, J. F., The Cauchy problem in a space of generalized functions I, C. R. Acad. Sci. Paris Sér. I Math., 317(1993), 851-855.

[CL] Colombeau, J.F.; Langlais, M., Generalized solutions of nonlinear parabolic equations with distributions as initial conditions, J. Math. Anal. Appl., 145 (1990), 186-196.

[CM] Colombeau, J.F.; Meril, A., Generalized functions and multiplication of distributions on $\mathscr{C}^{\infty}$ manifolds, J. Math. Anal. Appl., 186 (1994), 357-364. 
[Cor] Cordaro, P. D., Teoria das distribuições e análise de Fourier, Notas de aula da disciplina MAP5722, Universidade de São Paulo, São Paulo, 1999.

[CK] Cordaro, P. D.; Kawano, A., O delta de Dirac: uma introdução à teoria das distribuições para a engenharia, Editora Livraria da Física, São Paulo, 2002.

[Del] Delcroix, A., Remarks on the embedding of spaces of distributions into spaces of Colombeau generalized functions, preprint.

[Fer] Fernandez, R., A equação de Hamilton-Jacobi no contexto das funções generalizadas, Tese de Doutorado, Universidade de São Paulo, São Paulo, 1996.

[FS] Fichmann, L.; Sallum, E. M., Sistemas dinâmicos: noções básicas, Publicações do Instituto de Matemática e Estatística da Universidade de São Paulo, São Paulo, 2004.

[Gar-G] Garcia, A. R. G., Os números e as funções generalizadas plena de Colombeau: aspectos algébricos, topológicos e analíticos, Tese de Doutorado, Universidade de São Paulo, São Paulo, 2006.

[Gar-C-1] Garetto, C., Microlocal analysis in the dual of a Colombeau algebra: generalized wave front sets and noncharacteristic regularity, New York J. Math., 12 (2006), 275-318.

[Gar-C-2] Garetto, C., Pseudo-differential operators in algebras of generalized functions and global hypoellipticity, Acta Appl. Math., 80 (2004), 123-174.

[GKOS] Grosser, M.; Kunzinger, M.; Oberguggenberger, M.; Steinbauer, R., Geometric theory of generalized functions with applications to general relativity, Kluwer Acadademic Publishers, vol 537, Netherlands, 2001.

[Hör] Hörmander, L., The analysis of linear partial differential operators I: Distribution Theory and Fourier Analysis, Springer-Verlag, Berlin Heidelberg, 1983. 
[Jur] Juriaans, S. O., Uma introdução à teoria das funções generalizadas de Colombeau, Minicurso, 64 Seminário Brasileiro de Análise, 2006.

[KKO] Konjik, S.; Kunzinger, M.; Oberguggenberger, M., Foundations of the calculus of variations in generalized function algebras, Arxiv: 0707.1842v1 [math. FA] 12 jul 2007, 1-27.

[KO] Kunzinger, M.; Oberguggenberger, M., Characterization of Colombeau generalized functions by their point values, Math. Nachr., 203 (1999), 147-157.

[KOSV] Kunzinger, M.; Oberguggenberger, M.; Steinbauer, R.; Vickers, J. A., Generalized flows and singular ODEs on differentiable manifolds, Acta Appl. Math., 80 (2004), 221-241.

[KS] Kunzinger, M.; Steinbauer, R., Generalized pseudo-Riemannian geometry, Trans. Amer. Math. Soc., 354 (2002), 4179-4199.

[KSV] Kunzinger, M.; Steinbauer, R.; Vickers, J. A., Intrinsic characterization of manifold-valued generalized functions, Proc. London. Math. Soc., 87 (2003), 451-470.

[Lim] Lima, E. L., Curso de análise, vols 1, 2, Projeto Euclides, Instituto de Matemática Pura e Aplicada. CNPQ, Rio de Janeiro. 1976.

[Mil] Milies, F.C.P., Anéis e módulos, IME-USP, São Paulo, 1972.

[NPS] Nedeljkov, M.; Pilipović, S.; Scarpalezos, D., The linear theory of Colombeau generalized functions, Pitman Research Notes in Mathematics Series, 385, Longman, 1998.

[NOP] Nedeljkov, M.; Oberguggenberger, M.; Pilipović, S., Generalized solutions to a semilinear wave equation, Nonlinear Anal., 61 (2005), 461-475. 
[Obe] Oberguggenberger, M., Generalized functions and stochastics processes, Progress in Probability, 36 (1995), 215-229.

[OPS] Oberguggenberger, M.; Pilipović, S.; Scarpalézos, D., Positivity and positive definitiness in generalized function algebras, J. Math. Anal. Appl., 328 (2007), 1321-1335.

[OPV] Oberguggenberger, M.; Pilipović, S.; Valmorin, V., Global holomorphic representatives of Colombeau holomorphic generalized functions, preprint.

[Oli] Oliveira, E. R., Funções generalizadas em espaços de Banach. Um teorema do tipo Ovcyannikov-Treves e uma versão não linear do teorema de Ovcyannikov, Tese de Doutorado, Universidade de São Paulo, São Paulo, 2001.

[Pan] Panzarelli, H., Sobre a transformada de Hilbert no contexto das funções generalizadas, Tese de Doutorado, Universidade de São Paulo, São Paulo, 2004.

[Ros] Rosinger, E. E., Non-linear partial differential equations. An algebraic view of generalized solutions, North Holland, Amsterdam, 1990.

[RR] Rosinger, E. E.; Rudolph M., Group invariance of global generalized solutions of smooth nonlinear PDEs: a Dedekind order completion method, Lie Groups Appl., 1 (1994), 203-215.

[RW] Rosinger, E. E.; Walus, Y. E., Group invariance of generalized solutions obtained through the algebraic method, Nonlinearity, 7 (1994), 837-859.

[SC] Sansone, G.; Conti, R., Non-linear diferential equations, International series of monographs in Pure and Applied Mathematics, vol 67, The Macmillan Company, New York, 1964.

[Sca-1] Scarpalezos, D., Colombeau's generalized functions: topological structures microlocal properties. A simplified point of view, CNRS-URA212, Université Paris, 7, 1993. 
[Sca-2] Scarpalezos, D., Topologies dans les espaces de nouvelles fonctions generalisées de Colombeau. $\overline{\mathbb{C}}$-modules topologiques, Université Paris, 7, 1993.

[Sch] Schwartz, L., Théorie de distributions, I, II, 2nd ed., Herman, Paris, 1957.

[Sil-A] Silva, A. A., Sobre a álgebra simplificada das funções generalizadas de Colombeau, Dissertação de Mestrado, Universidade de São Paulo, São Paulo, 2005.

[Sil-T] Silva, J. T., Sobre a resolubilidade local de operadores diferenciais parciais lineares no contexto das funções generalizadas, Tese de Doutorado, Universidade de São Paulo, São Paulo, 1995.

[Sim] Simmons, G. F. Differential equations with applications and historical notes, McGraw-Hill Book Company, New York, 1972.

[Soa-R] Soares, M. R., Sobre alguns problemas da teoria das funções holomorfas generalizadas, Tese de Doutorado, Universidade de São Paulo, São Paulo, 2000.

[SV] Steinbauer, R.; Vickers, J. A., The use of generalized functions and distributions in general relativity, Classical Quantum Gravity, 23 (2006), 91-144.

[Tan] Tanabe, H., Equations of evolution, Monographs and Studies in Mathematics, vol 6, Pitman, London, 1979.

[Tro] Troutman, J. L., Variational calculus with elementary convexity, Springer Verlag, New York, 1983.

[Vil-1] Villarreal, F., Composition for a class of generalized functions in Colombeau's theory, Integral Transforms Spec. Funct., 11 (2001), 93-100.

[Vil-2] Villarreal, F., Heaviside generalized functions and shock waves for a Burgers kind equation, Integral Transforms Spec. Funct., 17 (2006), 213-219. 
[Vil-3] Villarreal, F., Sobre soluções na forma de onda de choque de certos sistemas de equações diferenciais parciais da hidrodinâmica, Tese de Doutorado, Universidade de São Paulo, São Paulo, 1990.

[Ver] Vernaeve, H., Embedding distributions in algebras of generalized functions with singularities, Monatsh. Math., 138 (2003), 307-318.

[Vei] Veiga, D. G., Análise real e complexa generalizada de Colombeau - uma introdução, Dissertação de mestrado, Universidade de São Paulo, São Paulo, 2007. 